\title{
Initial Assessment of the Soil and Vegetation of the Illinois National Guard Sparta Training Area
}

Jeffrey S. Fehmi, Robert L. McLeese, and June 2003 Jonathan L. Casebeer

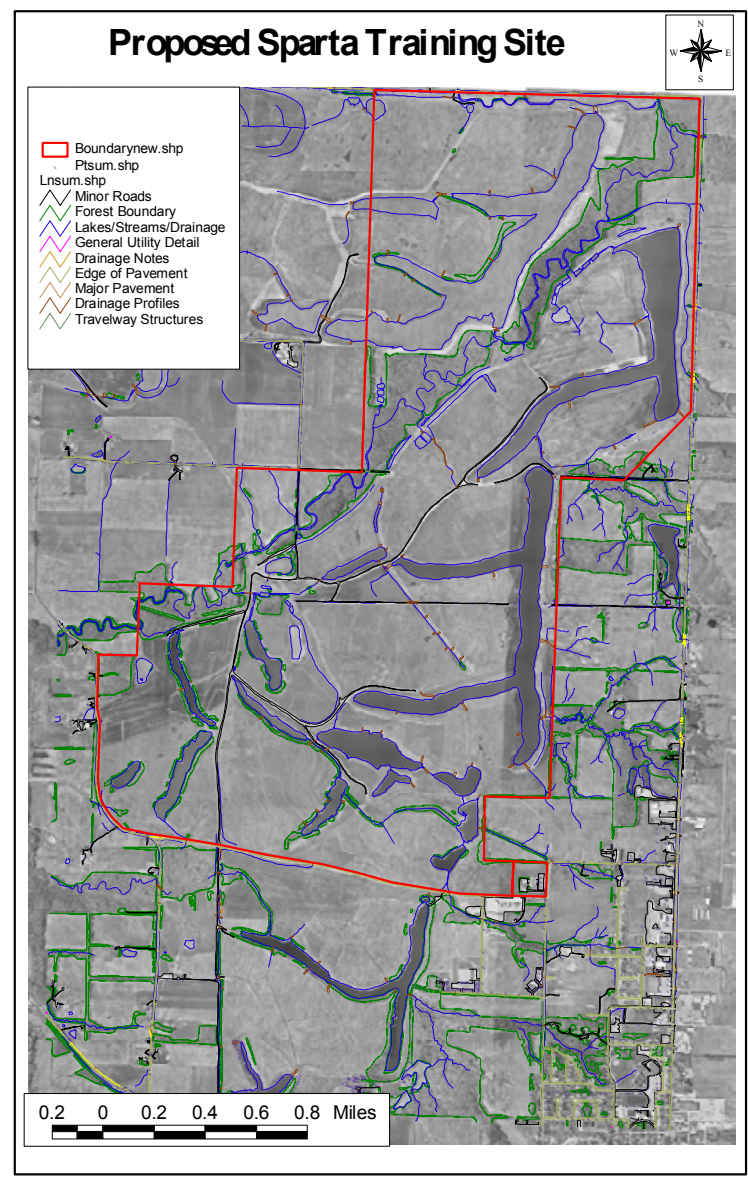





\section{Initial Assessment of the Soil and Vegetation of the Illinois National Guard Sparta Training Area}

Jeffrey S. Fehmi

Construction Engineering Research Laboratory

PO Box 9005

Champaign, IL 61826-9005

Robert L. McLeese

State Soil Scientist

USDA-NRCS

2118 W. Park Court

Champaign, IL 61821

Jonathan L. Casebeer

Military Environmental Specialist III

Illinois National Guard Camp Lincoln

1301 North MacArthur Blvd.

Springfield, IL 62702

Final Report

Approved for public release; distribution is unlimited.

Prepared for U.S. Army Corps of Engineers

Washington, DC 20314-1000

Under

Work Unit \#CNN-T081 
ABSTRACT: The Illinois National Guard (ILNG) is acquiring a new 2800-acre training area near Sparta, Illinois. This acquisition is important in that it allows the National Guard units in southern Illinois a readily available place to train, which will increase training effectiveness and save time and money through decreased travel costs associated with using the existing training area in the northern part of the state.

The recent acquisition of the Sparta training area represents a unique opportunity to gather baseline data before any training takes place. This data will be valuable in that it gives the Army the unique opportunity to learn about the conditions before and after training as well as strengthening any future empirically collected research data. This represents a fundamental knowledge gap in much of the current research on Army lands and represents a high priority, high payoff area of research.

The initial plant and soil data were collected using a grid-based sampling protocol to allow uniform and unbiased cover. The specific sampling protocols for each type of data follows in the vegetation and soils sections and the data are included in the appendices.

DISCLAIMER: The contents of this report are not to be used for advertising, publication, or promotional purposes. Citation of trade names does not constitute an official endorsement or approval of the use of such commercial products. All product names and trademarks cited are the property of their respective owners. The findings of this report are not to be construed as an official Department of the Army position unless so designated by other authorized documents.

DESTROY THIS REPORT WHEN NO LONGER NEEDED. DO NOT RETURN IT TO THE ORIGINATOR. 


\section{Contents}

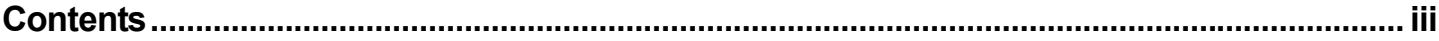

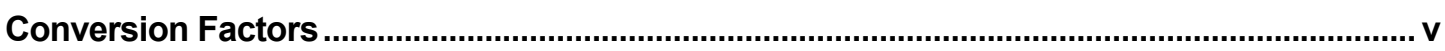

Preface

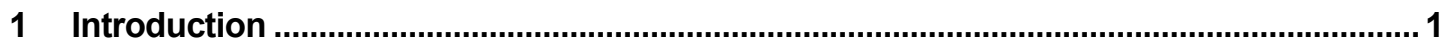

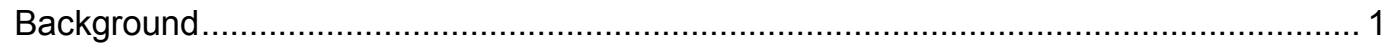

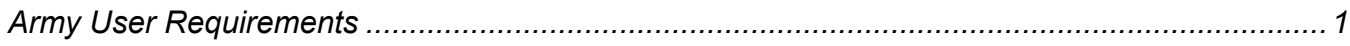

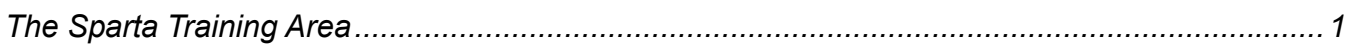

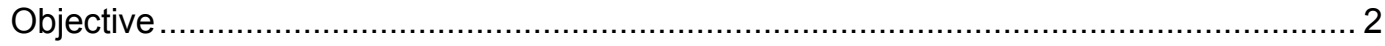

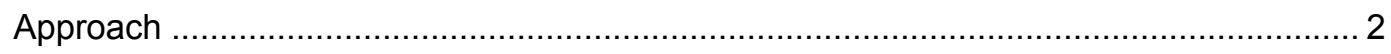

Scope

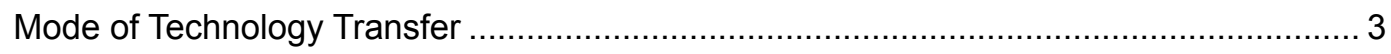

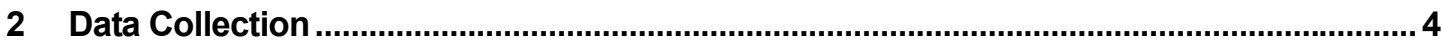

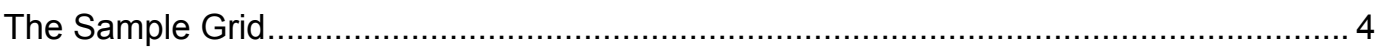

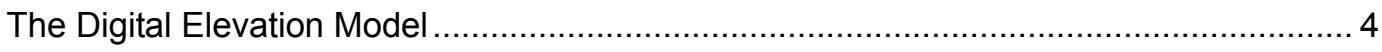

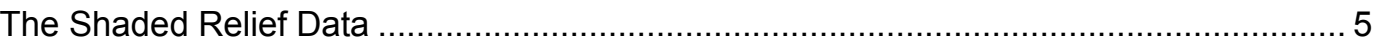

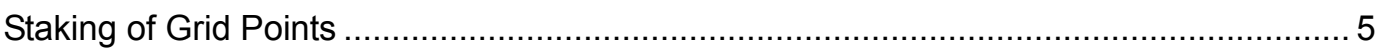

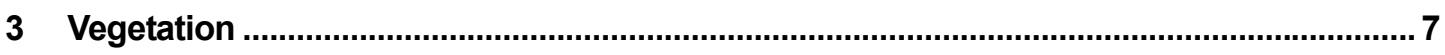

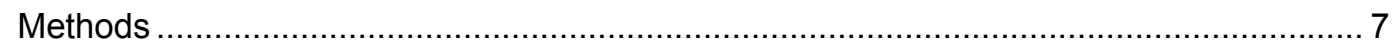

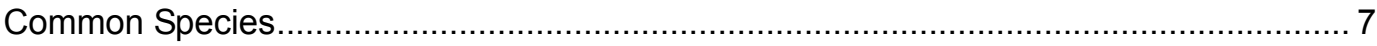

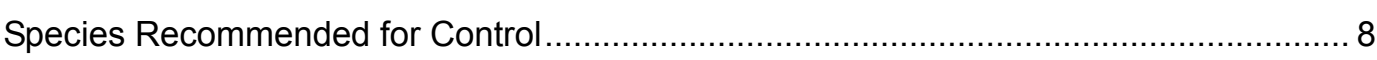

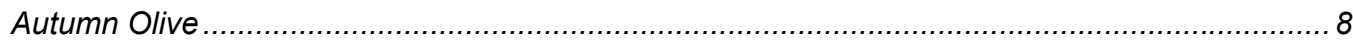

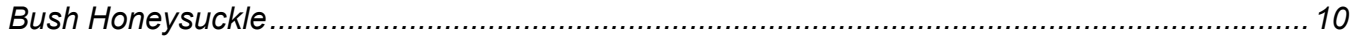

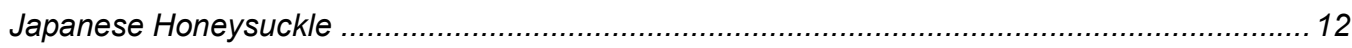

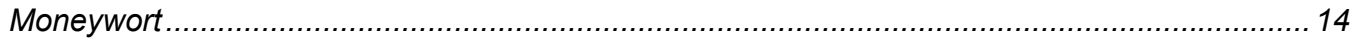

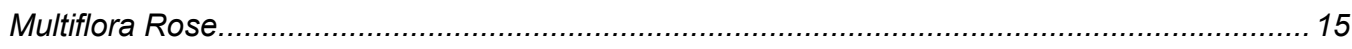

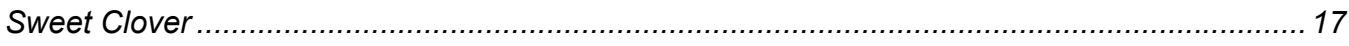

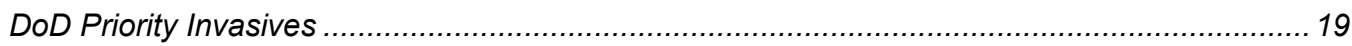

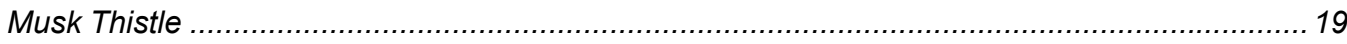

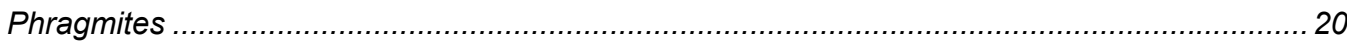

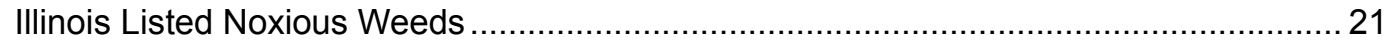


4 Soils

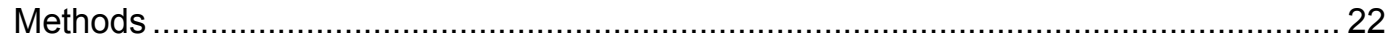

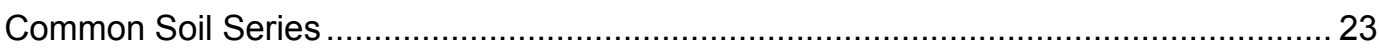

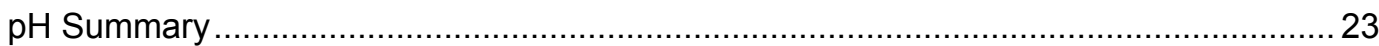

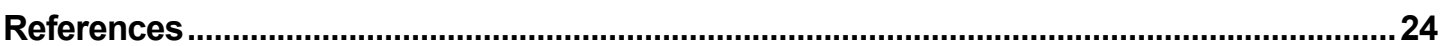

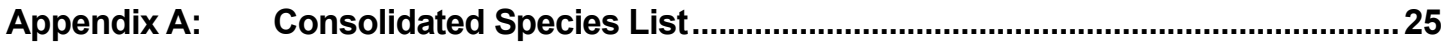

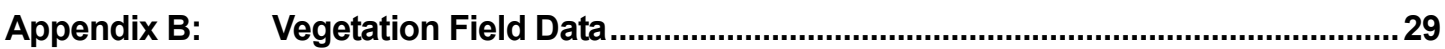

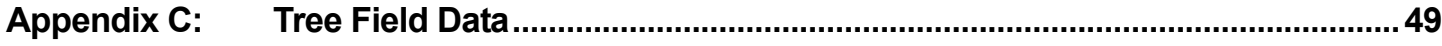

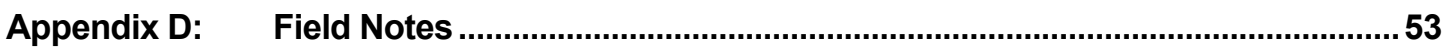

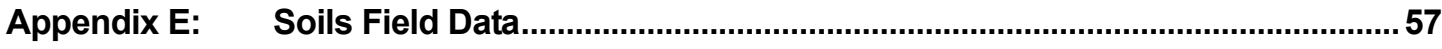

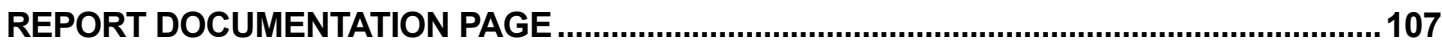




\section{Conversion Factors}

Non-SI units of measurement used in this report can be converted to SI units as follows:

\begin{tabular}{|c|c|c|}
\hline Multiply & By & To Obtain \\
\hline acres & $4,046.873$ & square meters \\
\hline cubic feet & 0.02831685 & cubic meters \\
\hline cubic inches & 0.00001638706 & cubic meters \\
\hline degrees (angle) & 0.01745329 & radians \\
\hline degrees Fahrenheit & $(5 / 9) \times\left({ }^{\circ} \mathrm{F}-32\right)$ & degrees Celsius \\
\hline degrees Fahrenheit & $(5 / 9) \times\left({ }^{\circ} \mathrm{F}-32\right)+273.15$ & kelvins \\
\hline feet & 0.3048 & meters \\
\hline gallons (U.S. liquid) & 0.003785412 & cubic meters \\
\hline horsepower (550 ft-lb force per second) & 745.6999 & watts \\
\hline inches & 0.0254 & meters \\
\hline kips per square foot & 47.88026 & kilopascals \\
\hline kips per square inch & 6.894757 & megapascals \\
\hline miles (U.S. statute) & 1.609347 & kilometers \\
\hline pounds (force) & 4.448222 & newtons \\
\hline pounds (force) per square inch & 0.006894757 & megapascals \\
\hline pounds (mass) & 0.4535924 & kilograms \\
\hline square feet & 0.09290304 & square meters \\
\hline square miles & $2,589,998$ & square meters \\
\hline tons (force) & $8,896.443$ & newtons \\
\hline tons $(2,000$ pounds, mass $)$ & 907.1847 & kilograms \\
\hline yards & 0.9144 & meters \\
\hline
\end{tabular}




\section{Preface}

This study was conducted for the Office of the Directorate of Environmental Programs (DAIM), Assistant Chief of Staff (Installation Management) (ACS[IM]) under project 622720896, "Environmental Quality Technology"; Work Unit CNNT081. The technical monitor was Dr. Vic Diersing, DAIM-ED-N.

The Construction Engineering Research Laboratory (CERL) Principal Investigator was Jeffrey S. Fehmi. The Illinois National Guard manager was Jonathan L. Casebeer. The work was performed by Robert McLeese, Dan Steinman, David Webber, Ellen Starr, Jerry Berning, Jon Bathgate, Matt McCauley, Paul Kremmel, and Roger Windhorn, all with the U.S. Department of Agriculture - Natural Resources Conservation Service and Illinois Department of Natural Resources District Forester, Mark Brown. Mr. Stephen Hodapp is Chief, CEERD-CN-N, and Dr. John T. Bandy is Chief, CEERD-CN. The associated Technical Director was Dr. William D. Severinghaus, CEERD-CV-T. The Director of CERL is Dr. Alan W. Moore.

CERL is an element of the U.S. Army Engineer Research and Development Center (ERDC), U.S. Army Corps of Engineers. The Commander and Executive Director of ERDC is COL John Morris III, EN and the Director of ERDC is Dr. James R. Houston. 


\section{Introduction}

\section{Background}

\section{Army User Requirements}

Documentation of the Army's environmental technology requirements has been an iterative process that began with a series of meetings in 1993 and the publication, U.S. Army Environmental Requirements and Needs from the Office of the Directorate of Environmental Programs. The Army's environmental technology requirements describe the critical research, development, test, and evaluation needs for accomplishing the Army's mission with the least impact or threat to the environment. These requirements are Army-level requirements that were reviewed for their impacts to readiness and quality of life, impact or threat to the environment, and timeliness needed for the Army to maintain compliance with environmental regulations. All major commands, major subcommands, the Office of the Deputy Chief of Staff for Operations, and the Office of the Deputy Chief of Staff for Logistics were involved in establishing the prioritized and validated list of the Army's environmental technology requirements.

Land Capacity and Characterization is the third priority conservation user requirement. This user requirement defines the Army's need to estimate training land carrying capacity. Twenty-eight exit criteria were identified in the Land Capacity and Characterization user requirement. Each exit criteria defines a specific product required to address a specific aspect of the overall requirement. Several of the exit requirements require detailed understanding of installation natural resources.

\section{The Sparta Training Area}

The Illinois National Guard (ILNG) is acquiring a new 2800-acre training area near Sparta, Illinois. This acquisition is important in that it allows the National Guard units in southern Illinois a readily available place to train, which will increase training effectiveness and save time and money through decreased travel costs associated with using the existing training area in the northern part of the state. 
The activities likely to take place at the Sparta Training Area include bivouac operations, assembly area and unit training of vehicle units, and various foottraffic type operations. The ILNG plans site improvements including an improved road network, hardening sites where substantial erosion would otherwise occur, and extensive tree planting to provide for tactical concealment areas. These improvements will increase the training realism and effectiveness and decrease any off-site impacts.

Prior to acquisition by the ILNG the site was used by the Peabody Coal Co. for coal extraction activities. After the mining activities had been completed, the site was rehabilitated with topsoil and vegetation. Before mining began, the land was used for various agricultural operations since about the 1830's. Before that time the land was a natural prairie system.

\section{Objective}

The recent acquisition of the Sparta training area represents a unique opportunity to gather baseline data before any training takes place. This data will be valuable in that it gives the Army the unique opportunity to learn about the conditions before and after training as well as strengthening any future empirically collected research data. This represents a fundamental knowledge gap in much of the current research on Army lands. Installation personnel and researchers from the Army as well as outside sources have corroborated that this kind of data represents a high priority, high payoff area of research.

\section{Approach}

The initial plant and soil data were collected using a grid-based sampling protocol. The grid-based protocol was chosen because it is difficult to predict the future use of any particular part of the site, current cover type, or current road network, since the ILNG plans to change the road network, increase tree cover, and set up a compartmental training schema. Having the sampling on a grid allows uniform and unbiased cover, which should capture multiple examples of any future common land uses. The specific sampling protocols for each type of data follows in the vegetation and soils sections and the data are included in the appendices. 


\section{Scope}

This report outlines the research and monitoring activities undertaken at the Illinois National Guard Sparta Training Area during 2001 and 2002. The general activities may be applicable to any Army site; however the specific research and monitoring apply only to the Sparta Training Area.

\section{Mode of Technology Transfer}

The data gathered during this project have been provided to the ILNG. It is also available to other land managers and research personnel.

This report will be made accessible through the World Wide Web (WWW) at URL:

http://www.cecer.army.mil 


\section{Data Collection}

\section{The Sample Grid}

The data collected in this study represent the site locations of the natural resource inventory conducted for the National Guard Sparta Training Area. Using a geographic information system (GIS), a 210-meter sampling grid was constructed to create 271 data collection points. At the time of this natural resource inventory, the proposed boundary for the site was not yet finalized, thus points were generated for all possible areas. Of the 271 points generated, only 212 fell inside the final property boundary and fell on land. (Point of contact: Jon Bathgate jon.bathgate@il.usda.gov.)

Vegetation and soils data were collected at each point (Figure 1). The purpose of the inventory was to provide baseline data to the National Guard on the natural resources of their newly acquired property.

\section{The Digital Elevation Model}

A digital elevation model (DEM) with a 2-meter horizontal resolution was constructed from 1-meter stereo plotter data provided by the Illinois National Guard. It was created with Arc Info's* TOPOGRID command using the Light Detection and Ranging (LIDAR) point data, a streams line coverage (digitized from Digital Orthophoto Quarter Quads [DOQQ]), and a lakes polygon coverage (also digitized from DOQQ).

*Arc Info is a product of ESRI, 380 New York St., Red Oak, CA 92373. 


\section{The Shaded Relief Data}

Color-painted, shaded relief data was created with a five-unit vertical exaggeration. It was created from the 2-meter DEM that was created from 1-meter stereo plotter data.

\section{Staking of Grid Points}

Points were selected for stakeout (Figure 1). A database file was obtained of the Universal Transverse Mercator (UTM) coordinates of each point for stakeout. This file was imported into AutoCAD ${ }^{*}{ }^{*}$ Land Desktop software. (Point of contact: David Webber David.Webber@il.usda.gov.)

* AutoCad is a product of Autodesk, Inc., 111 Mclnnis Parkway, San Rafael, CA 94903. 


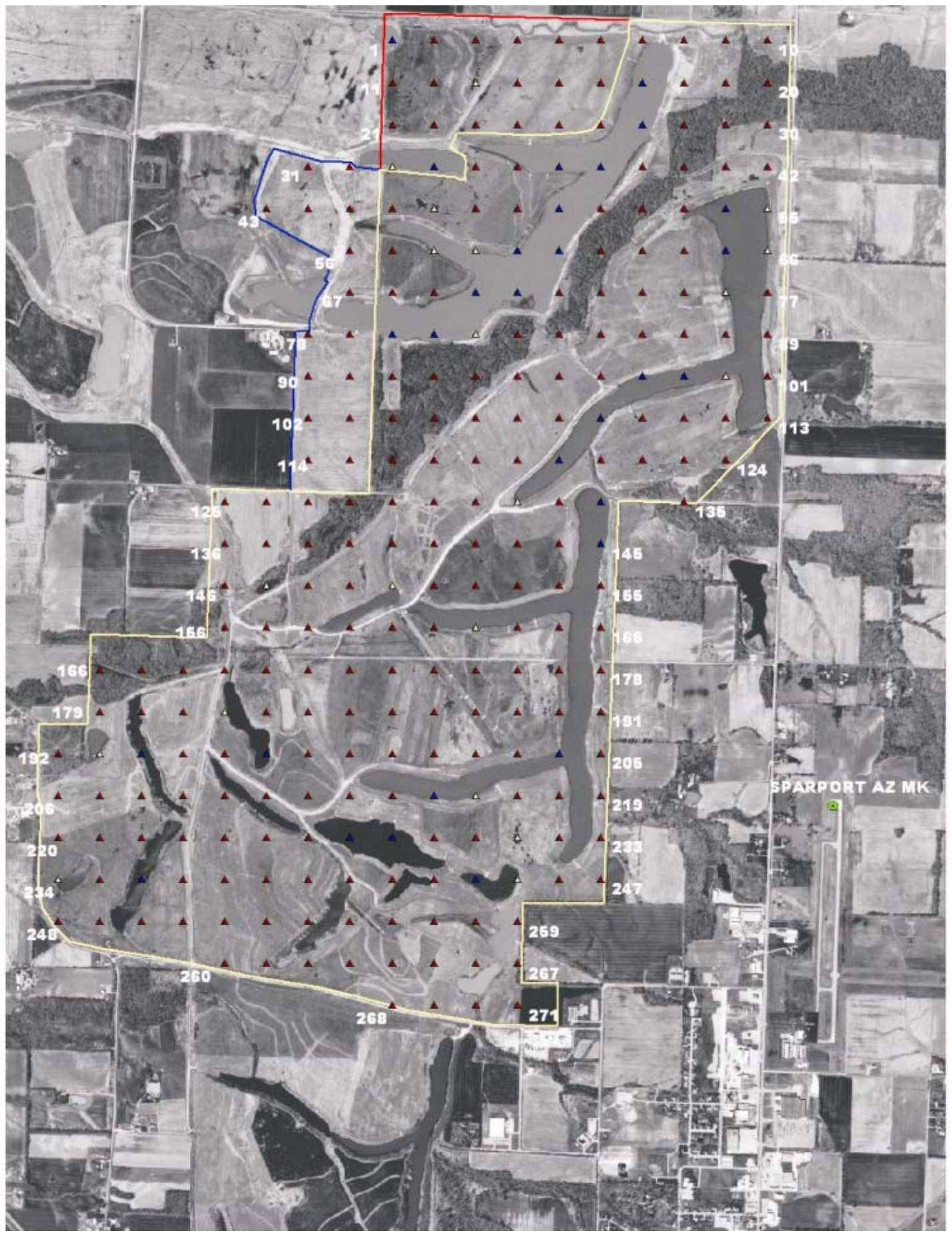

Figure 1. The sample points on an aerial photograph of the Sparta Training Area. 


\section{Vegetation}

\section{Methods}

The vegetation data were collected on the grid points shown in Figure 1 . The grid points are $210 \mathrm{~m}$ apart. The fieldwork was completed in May and June of 2002. The herbaceous vegetative survey was based on percent visual cover of the species composition adding up to 100 percent in a 2-meter square plot centered on the grid point. Appendix A contains the consolidated species list. Appendix B contains the vegetation field data. For this research, woody trees are larger than 20 feet tall and greater than 4 inches diameter at breast height (dbh). The IDNR District Forester, Mark Brown, took the tree measurements that provided basal composition using the $1 / 10$ acre prism method. Appendix C contains the tree data. All other fieldwork was done by Ellen Starr ellen.star@il.usda.gov. Additional field notes are in Appendix D.

\section{Common Species}

The species that occur on 10 or more of the sample plots include:

\begin{tabular}{|l|l|l|}
\hline Common name & Species & Number of plots \\
\hline Broomsedge & Andropogon virginicus & 10 \\
\hline Honey Locust & Gleditsia triacanthos & 10 \\
\hline Chickweed & Stellaria sp. & 10 \\
\hline American Elm & Ulmus americana & 10 \\
\hline Box Elder & Acer negundo & 11 \\
\hline Kentucky Bluegrass & Poa pratensis & 11 \\
\hline Virginia Wild Rye & Elymus virginicus & 13 \\
\hline Poison Ivy & Rhus radicans & 13 \\
\hline Sweet Clover & Melilotus officinalis & 14 \\
\hline Aster & Aster sp. & 15 \\
\hline Fox Sedge & Carex vulpinoidea & 15 \\
\hline Red Clover & Trifolium pratense & 17 \\
\hline Orchardgrass & Dactylis glomerata & 41 \\
\hline Goldenrod & Solidago sp. & 58 \\
\hline Fescue & Festuca arundinacea & 74 \\
\hline Smooth Brome & Bromus inermus & 91 \\
\hline
\end{tabular}




\section{Species Recommended for Control}

The species on the site that are recommended for control by the Illinois Nature Preserves Commission Vegetation Management Guideline (2002) include:

\begin{tabular}{|l|l|c|}
\hline Common name & Species & Number of plots \\
\hline Autumn Olive & Elaeagnus umbellata & 7 \\
\hline Bush Honeysuckle & Lonicera sp. & 2 \\
\hline Japanese Honeysuckle & Lonicera japonica & 3 \\
\hline Moneywort & Lysimachia nummularia & 1 \\
\hline Multiflora Rose & Rosa multiflora & 3 \\
\hline Sweet Clover & Melilotus officinalis & 14 \\
\hline Wild Carrot & Daucus carota & 4 \\
\hline
\end{tabular}

\section{Autumn Olive}

Excerpts from the Illinois Nature Preserves Commission Vegetation Management Guideline 2002, http://www.inhs.uiuc.edu/edu/VMG/VMG.html

Autumn olive was introduced into the United States in 1830 from its native range in China, Japan, and Korea. The strain 'Cardinal' was released in 1963 for commercial propagation. In the eastern and central United States, autumn olive has been planted primarily to provide food and cover for wildlife. It has also been planted as screens and barriers along highways, to stabilize and revegetate road banks, and to reclaim mine spoil. As late as 1975 this species was described as escaping rarely from cultivation. By 1981, it had been documented as naturalized in Illinois. Autumn olive has been officially recorded from only six counties; however, it is probably found in most counties now.

\section{Control practices in natural communities of high quality}

Young seedlings and sprouts can be handpulled in early spring when adequate ground moisture is present to allow removal of the root system along with aboveground growth. Autumn olive is easily seen in early spring because its leaves appear while most native vegetation is still dormant.

Cutting the plant off at the main stem and applying herbicide to the stump has been effective in killing root systems and preventing resprouting. Roundup herbicide (a formulation of glyphosate) has been effective in controlling autumn olive when used as a 10 to 20 percent solution and applied directly to the cut stump. Although the Roundup label specifies a higher concentration for cutstump application (50 to 100 percent), the lower concentration has proven effective. Roundup can be applied either by spraying individual stumps with a low- 
pressure hand-held sprayer or by wiping each stump using a sponge applicator. With cut-stump treatment, herbicide is applied specifically to the target plant, reducing the possibilities of damaging nearby, desirable vegetation. Cut-stump treatment is particularly effective late in the growing season (July through September), but is also effective during the dormant season. Glyphosate is a nonselective herbicide, so take care to avoid contacting nontarget species. By law, herbicides may be applied only according to label directions and by licensed herbicide applicators or operators when working on public properties.

\section{Control practices on buffer and severely disturbed sites}

In addition to the recommended control practices for high-quality natural communities, the following treatments are effective. Thin-line basal bark treatments with triclopyr herbicides (tradename Garlon) have demonstrated 95 percent kill. Undiluted Garlon 4 (or Garlon 4 diluted 50:50 with diesel fuel) should be applied in a thin line around the base of the plant 6 to 12 inches $(15$ to $30 \mathrm{~cm}$ ) above the ground. Application can be made with a hand-held plant sprayer and should be performed during the dormant season to minimize risk to nontarget species. To be effective, a narrow band of Garlon 4 needs to completely encircle the stem. Great care should be exercised to avoid getting any of the mixtures on the ground near the target plant since some nontarget species may be harmed. This method should not be used in high-quality natural areas because the diesel fuel may kill vegetation around the tree. Avoid using Triclopyr if rain is forecast for the following 1 to 4 days; otherwise runoff can harm nontarget species. By law, herbicides may be applied only according to label directions and by licensed herbicide applicators or operators when working on public properties.

Foliar application of dicamba herbicides (available under the tradename Banvel) and 2,4-D herbicides (available under a variety of brand names, including Crossbow) can provide total kill with little or no regrowth the following year. Banvel is mixed at the rate of 1 ounce per gallon of water plus 1/2 ounce of surfactant. The 2,4-D herbicide should be mixed according to label instructions. One hundred percent coverage of foliage should be achieved during the growing season (April through September). Although application can be done any time during the growing season, summer application (July through August) is especially effective. Banvel and 2,4-D are selective against broadleaf plants, so care must be taken to avoid contacting desirable, broadleaf vegetation. Do not spray so heav-

ily that herbicide drips off the target species. Foliar spray of herbicides should be used only in less sensitive areas because of problems with contacting nontarget species. The herbicide should be applied while backing away from treated areas to avoid walking through the wet herbicide. 
Although glyphosate (Roundup) is an effective foliar spray when applied during the growing season, it is not recommended because it is nonselective. Use of this herbicide as a foliar spray can result in unnecessary damage to nontarget species.

\section{Failed or ineffective practices}

Repeated pruning of established plants to ground level without subsequent herbicide application is not effective for controlling autumn olive. Each regrowth results in a thicker stem base and denser branches. Prescribed burning has not proven effective in controlling established autumn olive.

\section{Bush Honeysuckle}

Excerpts from the Illinois Nature Preserves Commission Vegetation Management Guideline 2002, http://www.inhs.uiuc.edu/edu/VMG/VMG.html

Bush honeysuckles are native to Asia and Western Europe. Tartarian honeysuckle was introduced to North America in 1752. The other species were introduced in the late 1800's and 1900's. Although their distribution is closely related to horticultural outlets, especially near larger urban areas where they are used as ornamentals, rural infestations are common when the species are used to improve wildlife habitat. In Illinois, the northern two-thirds of the state is the prime area of naturalization. Some localized outbreaks in southern and central Illinois have been noted. Although not recorded officially in many counties, bush honeysuckles are probably now found in most Illinois counties.

Control measures may include: prescribed burning, handpulling of seedlings, cutting, and herbicide treatments.

A recently introduced pest, the European Honeysuckle aphid, somewhat controls flower and fruit production in some of the bush honeysuckles. Heavy infestations cause tips of branches to form "witches brooms" or deformed twigs. This often greatly reduces fruit production. Native ladybug beetles, however, have been noted to control this aphid.

\section{Control practices in natural communities of high quality}

In fire-adapted communities, spring burning will kill seedlings and kill the tops of mature plants. Bush honeysuckles readily resprout and repeated fires are necessary for adequate control. It may be necessary to burn annually or biennially for 5 years or more for effective control. 
Seedlings may be pulled by hand when soils are moist. All of the root should be removed or resprouting will occur. Physical removal by pulling smaller plants or grubbing out large plants should not be used in sensitive habitats because open soil and remaining root stocks will result in rapid reinvasion or resprouting of honeysuckles and other exotics.

Bush honeysuckle stems can be cut at the base with brushcutters, chainsaws, or hand tools. The wood of bush honeysuckle is very tough and easily dulls powertool blades. After cutting, a 20 percent solution of glyphosate (Roundup or Rodeo) should be applied to the cut stump either by spraying the stump with a lowpressure hand-held sprayer or by wiping the herbicide on the stump with a sponge applicator to prevent resprouting. The Roundup and Rodeo labels recommend a 50 to 100 percent concentration of herbicide for stump treatment; however, a 20 percent concentration of Roundup has proven effective. It is not known if this lesser concentration is effective for Rodeo also. Rodeo can be used in wetlands and over open water, but Roundup is labeled for use only in nonwetlands. Herbicides should be applied to the cut stump immediately after cutting for best results. Application in late summer, early fall, or during the dormant season has proven effective. Because some resprouting may occur, follow-up treatment may be necessary. Glyphosate is nonselective, so take care to avoid contacting nontarget plants. By law, herbicides may be applied only according to label directions and by licensed herbicide applicators or operators when working on public properties.

\section{Control practices on buffer and severely disturbed sites}

Methods given above for high-quality natural communities are also effective and preferred on buffer and disturbed sites. When an area with bush honeysuckle lacks sufficient fuel to carry a fire, herbicides may be necessary to obtain control.

In dry, upland areas, a foliar spray of 1 percent Roundup (glyphosate) will control seedlings. A $1 \frac{1}{2}$ percent foliar spray of Roundup just after plant blooming in June will control mature shrubs. Application should occur from late June to just prior to leaf color changes in fall. The herbicide should be applied while backing away from treated areas so as not to walk through the wet herbicide.

In moist areas, a foliar spray of 1 percent Rodeo (glyphosate) with Ortho-X27 spreader, will control seedlings. Application should occur from late June to just prior to changes in leaf color in the fall. Foliar application of a $1 \frac{1}{2}$ percent solution of Rodeo will kill mature plants if all foliage is sprayed. This control method usually requires less labor but more herbicide. 
In addition, Krenite controls bush honeysuckle when applied according to label instructions.

Any treatment should be rechecked in following years for reinvasion. Glyphosate is a nonselective herbicide and care should be taken to avoid contacting nontarget plants with herbicide. Do not spray so heavily that herbicide drips off the target species. By law, herbicides may be applied only according to label instructions and by licensed herbicide applicators or operators when working on public properties.

\section{Failed or ineffective practices}

The herbicide Garlon does not control bush honeysuckle.

\section{Japanese Honeysuckle}

\section{Excerpts from the Illinois Nature Preserves Commission Vegetation Manage-} ment Guideline 2002, http://www.inhs.uiuc.edu/edu/VMG/VMG.html

Japanese honeysuckle, a native of Japan, was introduced to the United States in 1806 as a horticultural groundcover. It was slow to escape and did not become widely established over the eastern United States until the early 1900's. It has rapidly spread into many open natural communities in the southern two-thirds of Illinois. It has not been found to be a serious pest north of Peoria, although it is recorded from 10 northern Illinois counties. Bitter cold winter temperatures appear to limit this species somewhat. Nonetheless, this vine is becoming increasingly common in central Illinois.

\section{Control practices}

Efforts to control Japanese honeysuckle infestations have included the following methods: mowing, grazing, prescribed burning, and herbicides. While grazing and mowing reduce the spread of vegetative stems, prescribed burns or a combination of prescribed burns and herbicide spraying appears to be the best way to eradicate this vine.

In fire-adapted communities, spring prescribed burns greatly reduced Japanese honeysuckle coverage and crown volume. Repeated fires reduced honeysuckle by as much as 50 percent over a single burn. A previously burned population of honeysuckle will recover after several years if fire is excluded during this period. By reducing honeysuckle coverage with fire, refined herbicide treatments may be applied, if considered necessary, using less chemical. 
Because Japanese honeysuckle is semi-evergreen, it will continue to photosynthesize after surrounding deciduous vegetation is dormant. This condition allows managers to detect the amount of infestation, and allows for treatment of the infestation with herbicides without damage to the dormant vegetation.

Glyphosate herbicide (Roundup) is the recommended treatment for this honeysuckle. A 1.5 to 2 percent solution applied as a spray to the foliage will effectively eradicate Japanese honeysuckle. The herbicide should be applied after surrounding vegetation has become dormant in autumn and before a hard freeze $\left(25^{\circ} \mathrm{F}\right)$. Roundup should be applied carefully using a hand sprayer, and spray coverage should be uniform and complete. Do not spray so heavily that herbicide drips off the target species. Retreatment may be necessary for plants that are missed because of dense growth. Although glyphosate is effective when used during the growing season, use at this time is not recommended in natural areas because of the potential harm to nontarget plants. Glyphosate is nonselective, so care should be taken to avoid contacting nontarget species. Nontarget plants will be important in recolonizing the site after Japanese honeysuckle is controlled.

Crossbow, a formulation of triclopyr and 2,4-D, is also a very effective herbicide that controls Japanese honeysuckle. Crossbow should be mixed according to label instructions for foliar application and applied as a foliar spray. It may be applied at dormant periods, like glyphosate, and precautions given above for glyphosate should be followed when using Crossbow. Either herbicide should be applied while backing away from the treated area to avoid walking through the wet herbicide. By law, herbicides may be applied only according to label instructions and by licensed herbicide applicators or operators when working on public properties.

\section{Maintenance Control}

In fire-adapted communities, periodic spring burning should control this species.

\section{Failed or ineffective practices}

Mowing limits the length of Japanese honeysuckle vines, but will increase the number of stems produced. Grazing may have the same effects as mowing, but is less predictable due to uneven treatment given by browsing animals. Herbicides that have given poor control results or that are more persistent in the environment than other types are picloram, annitrole, aminotriazole, atrazine, dicamba, dicamba and 2,4-D, 2,4-D, DPX 5648, fenac, fenuron, simazine, and triclopyr. 


\section{Moneywort}

Excerpts from the Illinois Nature Preserves Commission Vegetation Management Guideline 2002, http://www.inhs.uiuc.edu/edu/VMG/VMG.html

This plant is a native of Great Britain and much of Europe. It was first introduced as an ornamental and was widespread from Georgia to Maine. It now can be found into Canada, throughout the north-central states, and along the west coast. It is distributed throughout Illinois. Moneywort invades floodplain forests, wet and mesic prairies, marshes, and swamps throughout the state. The plant tends to cover the ground with a mat of low-growing vegetation that excludes other herbaceous vegetation. Its ability to root at nodes enables it to cover large areas.

\section{Control practices in natural communities of high quality}

Generally, moneywort is not a major problem in high-quality communities. In low wetland woods where it is invading, one possible means of control is by prescribed burning in spring or fall when moneywort is green but most native vegetation is dormant. The plant can be handpulled where practical. All stems and stem fragments should be removed from the area to prevent the stems from rooting again in the soil.

\section{Control practices on buffer and severely disturbed sites}

In addition to the recommended control practices for high-quality natural communities, in low-quality buffer areas, prolonged submergence will kill moneywort. At restoration sites, moneywort can be controlled by establishing native grasses to shade it out. Suggested grasses include Cinna arundinacea and Elymus virginicus. Seeding of native grasses should be used only at restoration sites and not at natural areas. Herbicides such as Roundup or Rodeo may be effective control measures, but they have not been tested by Illinois natural area managers.

\section{Failed or ineffective practices}

Mowing is not effective since moneywort remains close to the ground due to its many rooting nodes. More research is needed concerning the effectiveness of herbicides. No biological controls that are feasible in natural areas are known. 


\section{Multiflora Rose}

\section{Excerpts from the Illinois Nature Preserves Commission Vegetation Manage- ment Guideline 2002, http://www.inhs.uiuc.edu/edu/VMG/VMG.html}

Multiflora rose was introduced into Illinois in the 1950's from eastern Asia as wildlife cover and food. Land managers recognized that plantings of this thorny, bushy shrub provided excellent escape cover and winter food. Because of its dense thorny nature, the commercial nursery trade began marketing it as a "living fence." The species soon spread and became a serious invader of agricultural lands, pastures, and natural communities throughout Illinois. It can form impenetrable thickets and smother out other vegetation. It is a serious pest species throughout the eastern United States.

\section{Control practices in natural communities of high quality}

Pulling, grubbing, or removing individual plants from the soil can be effective only when all roots are removed or when plants that develop subsequently from severed roots are destroyed. These approaches are most practical for light, scattered infestations.

In fire-adapted communities, a routine prescribed burn program will hinder invasion and establishment of multiflora rose.

Research indicates that 3 to 6 cuttings or mowings per growing season for more than 1 year can achieve high plant mortality. Such treatment may need to be repeated for 2 to 4 years. Increased mowing rates (more than 6 per season) did not increase plant mortality. In high-quality communities, repeated cutting is preferred over mowing, because repeated mowing will damage native vegetation as well as multiflora rose.

Cutting stems and either painting herbicide on the stump with a sponge applicator or spraying herbicide on the stump with a low-pressure hand-held sprayer kills root systems and prevents resprouting. Roundup herbicide (glyphosate) has been effective in controlling multiflora rose when used as a 10 to 20 percent solution and applied directly to the cut stump. Although the Roundup label recommends a higher concentration for cut-stump treatment (50 to 100 percent), the lower concentration has proven effective. With this technique, herbicide is applied specifically to the target plant, reducing the possibility of damaging nearby, desirable vegetation. Cut-stump treatment is effective late in the growing season (July through September), and also during the dormant season. Dormant season application is preferred because it will minimize potential harm to non- 
target species. Glyphosate is a nonselective herbicide, so take care to avoid contacting nontarget species. In addition, Triclopyr (tradename Garlon 3A) can be applied to cut stems or canes for selective control of multiflora rose. Garlon 3A diluted in water at a rate of 50 percent can be sprayed, using a hand-held sprayer, to the cut surface. Application should be within a few hours of cutting. Use of Garlon 3A is best done in the dormant season to lessen damage to nontarget species. Great care should be exercised to avoid getting any of the herbicide on the ground near the target plant since some nontarget species may be harmed. Avoid using Triclopyr if rain is forecast for the following 1 to 4 days; otherwise the runoff will harm nontarget species. By law, herbicides may be applied only according to label directions and by licensed herbicide applicators or operators when working on public properties.

\section{Control practices on buffer and severely disturbed sites}

Repeated cutting, as discussed above, is effective. For large populations on severely disturbed areas, mowing can be substituted for cutting individual plants. However, mowing multiflora rose can quickly flatten vehicle tires. Filling mower tires with foam is recommended.

Fosamine (tradename Krenite) can be applied as a foliar spray in a 2 percent solution plus 0.25 percent surfactant. The Krenite $\mathrm{S}$ formulation contains the appropriate amount of surfactant. Coverage of foliage should be complete. Krenite should be applied only in July through September. No effects will be observed during the autumn season following application. Slight regrowth may occur the following season but canes will die during summer. Fosamine kills only woody species and is nonvolatile; therefore it is the preferred foliar spray treatment.

Dicamba (tradename Banvel) is an effective foliar spray that is less preferred than Krenite. Banvel is selective against broadleaf plants, so care must be taken to avoid contacting desirable, broadleaf vegetation. It can be applied as a foliar spray in a 1 percent solution. Though this solution can be applied any time during the growing season, best results are obtained during May and June when plants are actively growing and flowering, following full leaf-out. One-half ounce of a surfactant should be added when treating dense foliage and, to enhance control in late season applications, complete coverage of all green leaves should be achieved. Do not spray Krenite or Dicamba so heavily that herbicide drips off the target species. Foliar spray of herbicides should be used only in less sensitive areas because of problems with contacting nontarget species. By law, herbicides may be applied only according to label directions and by licensed herbicide applicators or operators when working on public properties. 
Glyphosate (tradename Roundup) is an effective foliar spray when applied as a 1 percent solution to multiflora rose plants that are flowering or in bud. Roundup is not a preferred chemical treatment, however, because it is nonselective and the selective herbicides mentioned above are effective. Nevertheless, Roundup can be used as a foliar spray during the growing season on severely disturbed sites if care is taken to avoid contacting nontarget plants. Roundup should not be used as a foliar spray during the growing season in high-quality natural areas because it can result in damage to nontarget species. Roundup is useful as a foliar spray for alien plants that remain green and retain their leaves after native vegetation is dormant or senescent. Multiflora rose does not fit this description adequately and is controlled most effectively when treating during the growing season.

\section{Failed or ineffective practices}

No effective biological controls that are feasible in natural communities are known. Rose rosette is a sometimes fatal viral disease that attacks multiflora rose and other roses. This disease is not considered a useful biological control at this time because it may infect native roses and plums, as well as commercially important plants in the rose family such as apples, some types of berries, and ornamental roses.

\section{Sweet Clover}

\section{Excerpts from the Illinois Nature Preserves Commission Vegetation Manage- ment Guideline 2002, http://www.inhs.uiuc.edu/edu/VMG/VMG.html}

Sweet clover is native to Europe and Asia. It can be found in all 50 United States. The earliest records of its occurrence in North America date to 1664. More recently, around the turn of the century, sweet clover was cultivated as a forage crop and soil builder. Today it also is used as a wildlife cover crop and in the production of honey. Each species of sweet clover has been recorded from every county in Illinois, and adventive populations occur in disturbed habitats throughout most of the state. Since this exotic is considered economically important, and thus will continue to be planted, it will remain a problem for land managers well into the future.

\section{Control practices in natural communities of high quality}

Handpulling is effective if done when the ground is moist and most of the root can be removed. The best times to handpull sweet clover are in the late fall, after the first-year plant root-crown buds have developed, or anytime early in 
spring, before second-year plants develop flower buds. Fall weeding is recommended because: (1) the bright green sweet clover is easily spotted within the yellowing prairie, (2) moist fall conditions and an immature first-year root may make pulling easier, and (3) fall weeding is less stressful to native vegetation. However, sweet clover is easily located in the spring also, because it becomes green before native prairie vegetation. Handpulling in summer can be effective if done when the ground is moist. Handpulling is labor-intensive and must be done consistently. This treatment is feasible for light and moderate infestations, but may be too time consuming in heavy infestations.

In large, dense colonies of sweet clover, cutting first- and second-year stems close to the ground with a hand-held scythe is effective if done after leaves on the lower stems have died (before flowering occurs) and up to early stages of flowering (before seeds form). Sweet clover usually does not resprout when the stems are cut close to the ground during this time.

Prescribed burning can control sweet clover. A combination of an April burn in the first year, followed by a May burn the following year is most successful in eradicating an even-aged stand of sweet clover. A hot, complete, first-year April burn scarifies sweet clover seeds, stimulating them to grow (a late fall burn will also have this effect). A hot, complete, second-year May burn kills the emerging shoots before they can go to seed. Heavily infested stands are best controlled with the above sequence twice, separated by 2 years without burning. Problems with this method may arise if the burn is patchy, leaving viable seeds or secondyear shoots unscathed.

In an uneven-aged stand of sweet clover, second-year clover may escape the harmful effects of the early first-year burn because their shoots were not fully emerged. These plants would live to set seed. In this case, a combination of other procedures can be used. Spring burns could be later (after shoots emerge, but before second-year plants set seed) in a sequence of 3 to 5 years. You can also follow up the early burn with handpulling, if practical.

In an even-aged stand of sweet clover, fall mowing can speed up the 2-year burn program: burn in April; mow first-year plants in August, leaving the stems behind to dry; and burn again in mid-late September.

\section{Control practices on buffer and severely disturbed sites}

Control practices are the same as given above for high quality areas, with the following addition. Herbicide can be useful in controlling large sweet clover populations in degraded areas. Following a fall burn, hand-spray individual 
seedlings with an amine formulation of 2,4-D according to label instructions in spring, before native prairie vegetation emerges. This treatment also is effective when plants are in the cotyledon stage (i.e., when the first leaves appear in the development of the seedling). To reduce vapor drift, use an amine formulation of 2,4-D rather than an ester formulation. A 1 percent solution of Mecamine (2,4-D plus Dicamba) applied to the foliage as a spray is very effective. The herbicide 2,4-D amine is selective for broadleaf plants.

When applying either herbicide described above, spot application should be done such that coverage is uniform with the entire leaf being wet. Precautions should be taken to avoid contacting non-target plants with the solution. Do not spray so heavily that herbicide drips off the target species. By law, herbicides may be applied only according to label instructions and by licensed herbicide applicators or operators when working on public properties.

\section{Failed or ineffective practices}

No effective biological controls that are currently feasible in natural areas are known.

\section{DoD Priority Invasives}

Invasive plant species found on the Sparta Training Area that are high priority for DoD include:

\begin{tabular}{|l|l|c|}
\hline Common name & Scientific Name & Number of plots \\
\hline Multiflora Rose & Rosa multiflora & 3 \\
\hline Musk Thistle & Carduus nutans & 5 \\
\hline Phragmites & Phragmites australis & 1 \\
\hline
\end{tabular}

\section{Musk Thistle}

Excerpts from the Illinois Nature Preserves Commission Vegetation Management Guideline 2002, http://www.inhs.uiuc.edu/edu/VMG/VMG.html

A native of western Europe, musk thistle was introduced into the eastern United States in the early 1800's and has a long history as a rangeland pest. It was first discovered in Davidson County, Tennessee in 1942 and has been declared a noxious weed in many states. Because musk thistle is unpalatable to wildlife and livestock, selective grazing leads to severe degradation of native meadows and grasslands as wildlife focus their foraging on native plants, giving musk thistle a competitive advantage. Although musk thistle is infrequently found in dense 
forests, it can colonize areas subjected to natural disturbances such as landslides or frequent flooding. Meadows, prairies, grassy balds, and other open areas are susceptible to invasion.

Mechanical, biological, and chemical methods are effective and available for control of musk thistle. Handpulling is most effective on small populations and can be done throughout the year, but is most effective prior to the development of seeds. Flowers and seedheads should be bagged and disposed of in a landfill to prevent or minimize seed dispersal. Minimizing disturbance to the soil during removal activities will help reduce the chance of germination of seeds stored in the soil.

Two weevils have been introduced from Europe and released in the United States as a biological control for musk thistle: the thistlehead-feeding weevil (Rhinocyllus conicus) and the rosette weevil (Trichosirocalus horridus). These weevils have been released in a number of western states with some notable successes achieved. However, recent observations of unintentional and unanticipated impacts of the thistlehead-feeding weevil to native thistles, including some rare species, has raised a red flag about its continued use, at least in the western United States.

Foliar spraying is effective on established populations of musk thistle. Apply a 2 percent solution of glyphosate (Roundup) or triclopyr (Garlon) and water plus a 0.5 percent nonionic surfactant wetting all leaves and stems. Chlorpyralid (Transline) is effective at a concentration of 0.5 percent and is selective to Aster, Buckwheat, and Pea families. A low pressure and coarse spray pattern will limit drift and damage to nontarget species. Treatments should be applied during the rosette stage or prior to flowering. Glyphosate is a non-selective systemic herbicide that can kill nontarget plants that are only partially contacted by spray. Triclopyr is selective to broadleaf species and is a better choice if native grasses are present.

\section{Phragmites}

Excerpts from the Illinois Nature Preserves Commission Vegetation Management Guideline 200), http://www.inhs.uiuc.edu/edu/VMG/VMG.html

Common reed thrives in sunny wetland habitats. It grows along drier borders and elevated areas of brackish and freshwater marshes and along riverbanks and lakeshores. The species is particularly prevalent in disturbed or polluted soils found along roadsides, ditches, and dredged areas. Common reed has become a destructive weed, quickly displacing desirable plants species such as wild 
rice, cattails, and native plants. Invasive stands of common reed eliminate diverse wetland plant communities, and provide little food or shelter for wildlife.

Once established, common reed is very difficult to completely eradicate. However, careful planning and long-term management can yield varying levels of control. Herbicide use in combination with burning has generally proven to be the most effective means of control, and results in minimal disturbance to wetlands. Only a biodegradable herbicide that is licensed for use in wetlands and is nontoxic to animals can be used. Because a healthy wetland ecosystem is generally resistant to invasive species, long-term control of common reed depends on restoration of the health of the ecosystem.

\section{Illinois Listed Noxious Weeds}

The native plant, Common Ragweed, and the introduced Musk Thistle are listed Illinois noxious weeds (1994). The control measures for Ragweed and Wild Carrot (also know as Queen Anne's Lace) on natural areas were not available, but are likely to be similar to those recommended for other invasive plants. 


\section{Soils}

\section{Methods}

The soils data were collected on the grid points as described earlier. The fieldwork was completed in May and June of 2002. Of the 271 sites, several were in cropland where the flags had been removed for cultivation or were never placed knowing that they would be removed. Where there were no flags, a Rockwell PLUGGER was used to locate the site. At each site, a certified soil classifier employed by the Natural Resource Conservation Service completed a detailed description of the soil to a depth of 60 inches. The samples were collected using a Giddings probe and 2-inch diameter tube or a 1-inch diameter hand-driven probe. Where the site was not accessible by truck, the hand probe was used. Primary point of contact for this protocol and these data is Jerry Berning jerry.berning@il.usda.gov.

The Field Book for Describing and Sampling Soils, Version 1.1 was used to describe the color, texture, structure, consistence, $\mathrm{pH}$, and horizon boundaries. Hellige Reagent $\mathrm{N}$ Triplex Indicator was used to determine soil $\mathrm{pH}$ in the field. After the soil was described in the field, soil classifiers classified the soils to the series level when the soil characteristics fell within the range of characteristics of a defined soil series. Where the soil characteristics were not within the range of a soil series, the series most closely resembling the soil characteristics was assigned noting the soil characteristic that was outside the series range. Official soil series descriptions can be found at:

http://ortho.ftw.nrcs.usda.gov/osd/osd.html. The soils field data can be found in Appendix E. 


\section{Common Soil Series}

The two most common soil series on the site were Schuline and Lenzburg.

\begin{tabular}{|l|c|}
\hline Soil Series or land type & Percent of the site \\
\hline BIRDS & 5.7 \\
\hline OCONEE & 0.5 \\
\hline HAUL ROAD & 1.4 \\
\hline HICKORY & 1.4 \\
\hline HOSMER & 2.4 \\
\hline HURST & 0.5 \\
\hline LENZBURG & 17.2 \\
\hline LENZBURG (NON CALCAREOUS) & 2.4 \\
\hline MARINE & 1.9 \\
\hline MORRISTOWN & 2.4 \\
\hline RIPRAP & 0.5 \\
\hline SCHULINE & 45.2 \\
\hline SCHULINE (NON CALCAREOUS) & 8.6 \\
\hline STOY & 4.3 \\
\hline SWANWICK & 1.4 \\
\hline WAKELAND & 3.8 \\
\hline WILBUR & 0.5 \\
\hline
\end{tabular}

\section{pH Summary}

The soil profile $\mathrm{pH}$ varied from 3.5 to 9.5 with an average of about 6.5.

\begin{tabular}{|c|c|}
\hline $\mathrm{pH}$ value & Percent of the site \\
\hline $3.5-3.9$ & 0.23 \\
\hline $4.0-4.4$ & 0.00 \\
\hline $4.5-4.9$ & 0.23 \\
\hline $5.0-5.4$ & 1.85 \\
\hline $5.5-5.9$ & 15.92 \\
\hline $6.0-6.4$ & 23.18 \\
\hline $6.5-6.9$ & 9.69 \\
\hline $7.0-7.4$ & 10.38 \\
\hline $7.5-7.9$ & 16.72 \\
\hline $8.0-8.4$ & 18.22 \\
\hline $8.5-8.9$ & 0.00 \\
\hline $9.0-9.4$ & 0.00 \\
\hline $9.5-9.9$ & 0.12 \\
\hline
\end{tabular}




\section{References}

Barden, L.S. and J.F. Matthews. 1980. "Change in abundance of honeysuckle (Lonicera japnica) and other ground flora after prescribed burning of a piedmont pine forest," Castanea, 45:257-260.

Bureau of Environmental Programs. 1994. Illinois Noxious Weed Law and Rules. Illinois Department of Agriculture. http://plants.usda.gov/cgi_bin/state_noxious.cgi?statefips=17

Field Book for Describing and Sampling Soils by National Soil Survey Center, NRCS, Soil Survey Division Staff under the leadership of P.J. Schoeneberger, D.A. Wysocki, E.C. Benham, and W.D. Broderson, 1998. Version 2.0 is available at http://soils.usda.gov/procedures/field bk/main.htm

Illinois Nature Preserves Commission, 2002. Vegetation Management Guideline http://www.inhs.uiuc.edu/edu/VMG/VMG.html

Information about the high priority invasive species can be found at: http://www.invasivespecies.gov/profiles/main.shtml

Mclemore, B.F. 1981. "Evaluation of Chemicals for Controlling Japanese Honeysuckle.” Proc. South. Weed Sci. Soc. 34:298-210.

National Park Service Integrated Pest Management Manual, 2002. http://www.nature.nps.gov/wv/ipm/manual.html

Official soil series descriptions can be found at: http://ortho.ftw.nrcs.usda.gov/osd/osd.html 


\section{Appendix A: Consolidated Species List}

\begin{tabular}{|c|c|c|c|}
\hline Species & Scientific Name & $\begin{array}{l}\text { Number of } \\
\text { Sites }\end{array}$ & $\begin{array}{l}\text { Control } \\
\text { Recommended }\end{array}$ \\
\hline Ag. Field & $N A$ & 10 & \\
\hline Agrimony & Agrimonia sp. & 2 & \\
\hline Alfalfa & Medicago sativa & 5 & \\
\hline American Elm & Ulmus americana & 10 & \\
\hline Ash & Fraxinus sp. & 1 & \\
\hline Aster & Aster sp. & 15 & \\
\hline Autumn Olive & Elaeagnus umbellata & 7 & Yes $_{1}$ \\
\hline Bare Ground & $N A$ & 27 & \\
\hline Barley & Hordeum vulgare & 1 & \\
\hline Bedstraw & Galium sp. & 2 & \\
\hline Bitternut Hickory & Carya cordiformis & 2 & \\
\hline Black Raspberry & Rubus occidentalis & 1 & \\
\hline Black Snakeroot & Sanicula marilandica & 8 & \\
\hline Blackberry & Rubus allegheniensis & 1 & \\
\hline Blue Vervain & Verbena hastata & 4 & \\
\hline Blue-Eyed Mary & Collinsia verna & 5 & \\
\hline Box Elder & Acer negundo & 11 & \\
\hline Broomsedge & Andropogon virginicus & 10 & \\
\hline Bush Honeysuckle & Lonicera sp. & 2 & Yes $_{1}$ \\
\hline Buttercup & Ranunculus sp. & 2 & \\
\hline Butterweed & Senecio aureus & 2 & \\
\hline Canada Wild Rye & Elymus canadensis & 2 & \\
\hline Canada Wild Rye & Elymus cnandensis & 1 & \\
\hline Catbrier & Smilax sp. & 2 & \\
\hline Chickweed & Stellaria $s p$ & 10 & \\
\hline Cleavers & Galium aparine & 5 & \\
\hline Common Milkweed & Asclepias syriaca & 7 & \\
\hline Common Ragweed & Ambrosia artemisiifolia & 3 & $\mathrm{Yes}_{3}$ \\
\hline Coralberry & Symphoricarpos orbiculatus & 5 & \\
\hline Curled Dock & Rumex crispus & 6 & \\
\hline Dogwood Shrub & Cornus sp. & 2 & \\
\hline Downy Brome & Bromus tectorum & 1 & \\
\hline Dwarf Larkspur & Delphinium tricorne & 1 & \\
\hline Eastern Red Cedar & Juniperus virginiana & 2 & \\
\hline Exposed leaf litter & $N A$ & 1 & \\
\hline Fescue & Festuca arundinacea & 74 & \\
\hline
\end{tabular}




\begin{tabular}{|c|c|c|c|}
\hline Species & Scientific Name & $\begin{array}{l}\text { Number of } \\
\text { Sites }\end{array}$ & $\begin{array}{l}\text { Control } \\
\text { Recommended }\end{array}$ \\
\hline Flowering Dogwood & Cornus florida & 1 & \\
\hline Fox Sedge & Carex vulpinoidea & 15 & \\
\hline Foxglove Beardtongue & Penstemon digitalis & 1 & \\
\hline Foxtail & Setaria sp. & 1 & \\
\hline Goldenrod & Solidago sp. & 58 & \\
\hline Grapevine & Vitis sp. & 8 & \\
\hline Gravel & $N A$ & 2 & \\
\hline Green Ash & Fraxinus pennsylvanica & 8 & \\
\hline Green Bulrush & Scirpus atrovirens & 1 & \\
\hline Hackberry & Celtis occidentalis & 7 & \\
\hline Hawthorn & Crataegus sp. & 1 & \\
\hline Henbit & Lamium amplexicaule & 2 & \\
\hline Honewort & Cryptotaenia canadensis & 3 & \\
\hline Honey Locust & Gleditsia triacanthos & 10 & \\
\hline Horseweed & Erigeron canadensis & 4 & \\
\hline Indian Hemp & Apocynum cannabinum & 1 & \\
\hline Japanese Honeysuckle & Lonicera japonica & 3 & Yes $_{1}$ \\
\hline Jewelweed & Impatiens capensis & 5 & \\
\hline Jimsonweed & Datura stramonium & 1 & \\
\hline Kentucky Bluegrass & Poa pratensis & 11 & \\
\hline Korean Lespedeza & Lespedeza stipulacea & 1 & \\
\hline Ladino Clover & Trifolium repens latum & 4 & \\
\hline Late-Flowering Thoroughwort & Eupatorium serotinum & 6 & \\
\hline Late-Flowering Thouroughwort & Eupatorium serotinum & 1 & \\
\hline Lespedeza & Lespedeza sp. & 7 & \\
\hline May Apple & Podophyllum peltatum & 3 & \\
\hline Mixed Weeds & $N A$ & 2 & \\
\hline Moneywort & Lysimachia nummularia & 1 & Yes $_{1}$ \\
\hline Multiflora Rose & Rosa multiflora & 3 & Yes $_{1,2}$ \\
\hline Musk Thistle & Carduus nutans & 5 & Yes $_{2,3}$ \\
\hline Narrow-leaved Cattail & Typha angustifolia & 1 & \\
\hline Oats & Avena sativa & 9 & \\
\hline Orchardgrass & Dactylis glomerata & 41 & \\
\hline Panicgrass & Dicanthelium sp. & 1 & \\
\hline Pennsylvania Bittercress & Cardamine pensylvanica & 1 & \\
\hline Perennial Rye & Lolium perenne & 4 & \\
\hline Persimmon & Diospyros virginiana & 1 & \\
\hline Phlox & Phlox sp. & 4 & \\
\hline Phragmites & Phragmites australis & 1 & $\mathrm{Yes}_{2}$ \\
\hline Pigweed & Amaranthus sp. & 4 & \\
\hline Pin Oak & Quercus palustris & 3 & \\
\hline Poison Ivy & Rhus radicans & 13 & \\
\hline Pokeweed & Phytolacca americana & 2 & \\
\hline Prickly Brambles & Rubus sp. & 3 & \\
\hline Red Clover & Trifolium pratense & 17 & \\
\hline
\end{tabular}




\begin{tabular}{|c|c|c|c|}
\hline Species & Scientific Name & $\begin{array}{l}\text { Number of } \\
\text { Sites }\end{array}$ & $\begin{array}{l}\text { Control } \\
\text { Recommended }\end{array}$ \\
\hline Red Trillium & Trillium erectum & 1 & \\
\hline Residue Covered Ground & $N A$ & 51 & \\
\hline \multicolumn{4}{|c|}{ Residue Covered Ground and Bare } \\
\hline Ground & $N A$ & 10 & \\
\hline Rip-Rap & $N A$ & 1 & \\
\hline River Birch & Betula nigra & 1 & \\
\hline Rush & Juncus sp. & 8 & \\
\hline Sedge & Carex sp. & 9 & \\
\hline Sericea & Sericea lespedeza & 1 & \\
\hline Shellbark Hickory & Carya laciniosa & 5 & \\
\hline Shepard's-purse & Capsella bursa-pastoris & 1 & \\
\hline Silver Maple & Acer saccharinum & 4 & \\
\hline Slender Rush & Juncus sp. & 1 & \\
\hline Smartweed & Polygonum pensylvanicum & 4 & \\
\hline Smooth Brome & Bromus inermus & 91 & \\
\hline Solomon's Seal & Smilacina sp. & 2 & \\
\hline Soybeans & Glycine max & 5 & \\
\hline Spring Beauty & Claytonia virginica & 5 & \\
\hline St. John's Wort & Hypericum sp. & 1 & \\
\hline Stinging Nettle & Urtica dioica & 6 & \\
\hline Stout Woodreed & Cinna arundinacea & 2 & \\
\hline Sugarberry & Celtis laevigata & 4 & \\
\hline Sumac & Rhus sp. & 2 & \\
\hline Swamp White Oak & Quercus bicolor & 2 & \\
\hline Sweet Cicely & Osmorhiza longistylis & 7 & \\
\hline Sweet Clover & Melilotus sp. & 3 & Yes $_{1}$ \\
\hline Sweet White Clover & Melilotus alba & 3 & \\
\hline Sweet William & Phlox maculata & 2 & \\
\hline Sweet Yellow Clover & Melilotus officinalis & 11 & Yes $_{1}$ \\
\hline Sycamore & Platanus occidentalis & 2 & \\
\hline Timothy & Phleum pratense & 5 & \\
\hline Trout Lily & Erythronium americanum & 1 & \\
\hline Trumpet Creeper & Campsis radicans & 9 & \\
\hline Tumble mustard & Sisymbrium altissimum & 1 & \\
\hline Virginia Bluebell & Mertensia virginica & 2 & \\
\hline Virginia Creeper & Parthenosensis quinqifolia & 3 & \\
\hline Virginia Wild Rye & Elymus virginicus & 13 & \\
\hline Wet bare ground & $N A$ & 1 & \\
\hline Wheat & Triticum aestivum & 5 & \\
\hline White Clover & Trifolium repens & 6 & \\
\hline Wild Carrot & Daucus carota & 4 & $\mathrm{Yes}_{2}$ \\
\hline Wild Geranium & Geranium maculatum & 2 & \\
\hline Wild Onion & Allium canadense & 8 & \\
\hline Willow & Salix sp. & 1 & \\
\hline Winter Wheat & Triticum aestivum & 2 & \\
\hline
\end{tabular}




\begin{tabular}{llll} 
& & \multicolumn{2}{c}{ Number of Control } \\
Species & Scientific Name & Sites & Recommended \\
\hline Wood-Sorrel & Oxalis sp. & 2 \\
Woolgrass & Scirpus cyperinus & 1 \\
Yarrow & Achillea millefolium & 7 \\
Yellow Violet & Viola pensylvanica & 7
\end{tabular}

1. Recommended for control by the Illinois Nature Preserves Commission, 2002. Vegetation Management Guide [online] http://www.inhs.uiuc.edu/edu/VMG/VMG.html

2. High priority invasive plant species for DoD. [online]

http://www.invasivespecies.gov/profiles/main.shtml

3. Bureau of Environmental Programs. 1994. Illinois Noxious Weed Law and Rules. Illinois Department of Agriculture. [online] http://plants.usda.gov/cgi bin/state noxious.cgi?statefips=17 


\section{Appendix B: Vegetation Field Data}

\begin{tabular}{|c|c|c|c|c|}
\hline SITE & COMMON NAME & SCIENTIFIC NAME & COVER & STRATUM \\
\hline 7 & Goldenrod & Solidago sp. & 70 & $\mathrm{H}$ \\
\hline 7 & Sweet White Clover & Melilotus alba & 20 & $\mathrm{H}$ \\
\hline 7 & Canada Wild Rye & Elymus cnandensis & 10 & $\mathrm{H}$ \\
\hline 7 & Green Ash & Fraxinus pennsylvanica & 60 & $S$ \\
\hline 8 & Fescue & Festuca arundinacea & 85 & $\mathrm{H}$ \\
\hline 8 & Timothy & Phleum pratense & 5 & $\mathrm{H}$ \\
\hline 8 & Goldenrod & Solidago sp. & 10 & $\mathrm{H}$ \\
\hline 8 & Honey Locust & Gleditsia triacanthos & 70 & S \\
\hline 9 & Orchardgrass & Dactylis glomerata & 70 & $\mathrm{H}$ \\
\hline 9 & Agrimony & Agrimonia sp. & 20 & $\mathrm{H}$ \\
\hline 9 & Goldenrod & Solidago sp. & 10 & $\mathrm{H}$ \\
\hline 9 & Coralberry & Symphoricarpos orbiculatus & 10 & S \\
\hline 9 & Box Elder & Acer negundo & 5 & S \\
\hline 9 & Ash & Fraxinus sp. & 50 & $S$ \\
\hline 10 & Smooth Brome & Bromus inermus & 90 & $\mathrm{H}$ \\
\hline 10 & Smartweed & Polygonum pensylvanicum & 5 & $\mathrm{H}$ \\
\hline 10 & Wild Onion & Allium canadense & 5 & $\mathrm{H}$ \\
\hline 18 & Fescue & Festuca arundinacea & 70 & $\mathrm{H}$ \\
\hline 18 & Sweet Yellow Clover & Melilotus officinalis & 20 & $\mathrm{H}$ \\
\hline 18 & Goldenrod & Solidago sp. & 10 & $\mathrm{H}$ \\
\hline 19 & Japanese Honeysuckle & Lonicera japonica & 60 & v \\
\hline 19 & Goldenrod & Solidago sp. & 30 & $\mathrm{H}$ \\
\hline 19 & Trumpet Creeper & Campsis radicans & 30 & $\mathrm{H}$ \\
\hline 19 & Indian Hemp & Apocynum cannabinum & 20 & $\mathrm{H}$ \\
\hline 19 & Blue Vervain & Verbena hastata & 20 & $\mathrm{H}$ \\
\hline 19 & Box Elder & Acer negundo & 10 & s \\
\hline 19 & Green Ash & Fraxinus pennsylvanica & 10 & S \\
\hline 19 & Autumn Olive & Elaeagnus umbellata & 70 & S \\
\hline 19 & American Elm & Ulmus americana & 10 & S \\
\hline 20 & Blue-Eyed Mary & Collinsia verna & 35 & $\mathrm{H}$ \\
\hline
\end{tabular}

Data collected in May and June 2002. H=herbaceous, $S=$ shrub, V=vine and $G=$ ground. Plots were centered on the points. Plots containing shrubs or vines may add up to more than $100 \%$ cover (visually estimated) due to the presence of both herbaceous and shrub canopies. 


\begin{tabular}{|c|c|c|c|c|}
\hline SITE & COMMON NAME & SCIENTIFIC NAME & COVER & STRATUM \\
\hline 20 & Sweet Cicely & Osmorhiza longistylis & 45 & $\mathrm{H}$ \\
\hline 20 & Virginia Wild Rye & Elymus virginicus & 15 & $\mathrm{H}$ \\
\hline 20 & Henbit & Lamium amplexicaule & 3 & $\mathrm{H}$ \\
\hline 20 & Chickweed & Stellaria $s p$ & 2 & $\mathrm{H}$ \\
\hline 20 & Coralberry & Symphoricarpos orbiculatus & 3 & $\mathrm{~S}$ \\
\hline 20 & Box Elder & Acer negundo & 10 & $S$ \\
\hline 20 & Shellbark Hickory & Carya laciniosa & 10 & $S$ \\
\hline 20 & Pin Oak & Quercus palustris & 5 & $S$ \\
\hline 20 & Grapevine & Vitis sp. & 2 & V \\
\hline 26 & Smooth Brome & Bromus inermus & 85 & $\mathrm{H}$ \\
\hline 26 & Residue Covered Ground & $N A$ & 15 & G \\
\hline 28 & Sweet Cicely & Osmorhiza longistylis & 70 & $\mathrm{H}$ \\
\hline 28 & Virginia Wild Rye & Elymus virginicus & 35 & $\mathrm{H}$ \\
\hline 28 & Virginia Bluebell & Mertensia virginica & 5 & $\mathrm{H}$ \\
\hline 28 & Bedstraw & Galium sp. & 25 & $\mathrm{H}$ \\
\hline 28 & Grapevine & Vitis sp. & 2 & $\mathrm{~V}$ \\
\hline 28 & Blue-Eyed Mary & Collinsia verna & 5 & $\mathrm{H}$ \\
\hline 28 & Yellow Violet & Viola pensylvanica & 5 & $\mathrm{H}$ \\
\hline 28 & Swamp White Oak & Quercus bicolor & 2 & S \\
\hline 28 & Poison Ivy & Rhus radicans & 5 & $\mathrm{H}$ \\
\hline 28 & Hackberry & Celtis occidentalis & 2 & S \\
\hline 28 & Coralberry & Symphoricarpos orbiculatus & 1 & $S$ \\
\hline 29 & Virginia Wild Rye & Elymus virginicus & 50 & $\mathrm{H}$ \\
\hline 29 & Stout Woodreed & Cinna arundinacea & 20 & $\mathrm{H}$ \\
\hline 29 & Solomon's Seal & Smilacina sp. & 2 & $\mathrm{H}$ \\
\hline 29 & Sweet Cicely & Osmorhiza longistylis & 10 & $\mathrm{H}$ \\
\hline 29 & Spring Beauty & Claytonia virginica & 25 & $\mathrm{H}$ \\
\hline 29 & Phlox & Phlox sp. & 5 & $\mathrm{H}$ \\
\hline 29 & Poison Ivy & Rhus radicans & 5 & $\mathrm{H}$ \\
\hline 29 & Poison Ivy & Rhus radicans & 2 & V \\
\hline 29 & Trumpet Creeper & Campsis radicans & 2 & V \\
\hline 29 & Black Raspberry & Rubus occidentalis & 2 & S \\
\hline 29 & Coralberry & Symphoricarpos orbiculatus & 5 & S \\
\hline 29 & Shellbark Hickory & Carya laciniosa & 2 & S \\
\hline 29 & Hackberry & Celtis occidentalis & 5 & $\mathrm{~S}$ \\
\hline 29 & Dwarf Larkspur & Delphinium tricorne & 2 & $\mathrm{H}$ \\
\hline 29 & Yellow Violet & Viola pensylvanica & 5 & $\mathrm{H}$ \\
\hline 30 & Soybeans & Glycine max & 100 & $\mathrm{H}$ \\
\hline 31 & Ag. Field & $N A$ & 100 & $\mathrm{H}$ \\
\hline 32 & Ag. Field & $N A$ & 100 & $\mathrm{H}$ \\
\hline 35 & Smooth Brome & Bromus inermus & 85 & $\mathrm{H}$ \\
\hline 35 & Sweet White Clover & Melilotus alba & 5 & $\mathrm{H}$ \\
\hline 35 & Goldenrod & Solidago sp. & 5 & $\mathrm{H}$ \\
\hline 35 & Yarrow & Achillea millefolium & 5 & $\mathrm{H}$ \\
\hline
\end{tabular}




\begin{tabular}{|c|c|c|c|c|}
\hline SITE & COMMON NAME & SCIENTIFIC NAME & COVER & STRATUM \\
\hline 36 & Smooth Brome & Bromus inermus & 100 & $\mathrm{H}$ \\
\hline 36 & Honey Locust & Gleditsia triacanthos & 30 & $S$ \\
\hline 39 & Rush & Juncus sp. & 40 & $\mathrm{H}$ \\
\hline 39 & Fox Sedge & Carex vulpinoidea & 25 & $\mathrm{H}$ \\
\hline 39 & Smartweed & Polygonum pensylvanicum & 5 & $\mathrm{H}$ \\
\hline 39 & Late-Flowering Thoroughwort & Eupatorium serotinum & 5 & $\mathrm{H}$ \\
\hline 39 & Goldenrod & Solidago sp. & 25 & $\mathrm{H}$ \\
\hline 39 & Green Ash & Fraxinus pennsylvanica & 20 & S \\
\hline 39 & Silver Maple & Acer saccharinum & 5 & S \\
\hline 40 & Sweet Cicely & Osmorhiza longistylis & 60 & $\mathrm{H}$ \\
\hline 40 & Black Snakeroot & Sanicula marilandica & 40 & $\mathrm{H}$ \\
\hline 40 & Spring Beauty & Claytonia virginica & 20 & $\mathrm{H}$ \\
\hline 40 & Virginia Wild Rye & Elymus virginicus & 20 & $\mathrm{H}$ \\
\hline 40 & Yellow Violet & Viola pensylvanica & 10 & $\mathrm{H}$ \\
\hline 40 & Blue-Eyed Mary & Collinsia verna & 2 & $\mathrm{H}$ \\
\hline 40 & Poison Ivy & Rhus radicans & 1 & $\mathrm{H}$ \\
\hline 40 & Bitternut Hickory & Carya cordiformis & 2 & S \\
\hline 40 & Shellbark Hickory & Carya laciniosa & 2 & S \\
\hline 40 & Hawthorn & Crataegus sp. & 2 & s \\
\hline 40 & Hackberry & Celtis occidentalis & 2 & S \\
\hline 40 & Grapevine & Vitis sp. & 1 & V \\
\hline 40 & May Apple & Podophyllum peltatum & 1 & $\mathrm{H}$ \\
\hline 41 & Aster & Aster sp. & 10 & $\mathrm{H}$ \\
\hline 41 & Goldenrod & Solidago sp. & 10 & $\mathrm{H}$ \\
\hline 41 & Broomsedge & Andropogon virginicus & 10 & $\mathrm{H}$ \\
\hline 41 & Trumpet Creeper & Campsis radicans & 10 & $\mathrm{H}$ \\
\hline 41 & Bare Ground & $N A$ & 60 & $G$ \\
\hline 42 & Goldenrod & Solidago sp. & 30 & $\mathrm{H}$ \\
\hline 42 & Sedge & Carex sp. & 60 & $\mathrm{H}$ \\
\hline 42 & Wild Onion & Allium canadense & 10 & $\mathrm{H}$ \\
\hline 42 & Sycamore & Platanus occidentalis & 80 & S \\
\hline 42 & Box Elder & Acer negundo & 10 & s \\
\hline 42 & Persimmon & Diospyros virginiana & 10 & S \\
\hline 43 & Soybeans & Glycine max & 15 & $\mathrm{H}$ \\
\hline 43 & Wild Onion & Allium canadense & 10 & $\mathrm{H}$ \\
\hline 43 & Wheat & Triticum aestivum & 10 & $\mathrm{H}$ \\
\hline 43 & Bare Ground & $N A$ & 65 & $G$ \\
\hline 44 & Soybeans & Glycine max & 15 & $\mathrm{H}$ \\
\hline 44 & Wild Onion & Allium canadense & 10 & $\mathrm{H}$ \\
\hline 44 & Wheat & Triticum aestivum & 10 & $\mathrm{H}$ \\
\hline 44 & Bare Ground & $N A$ & 65 & G \\
\hline 45 & Soybeans & Glycine max & 15 & $\mathrm{H}$ \\
\hline 45 & Wild Onion & Allium canadense & 10 & $\mathrm{H}$ \\
\hline 45 & Wheat & Triticum aestivum & 10 & $\mathrm{H}$ \\
\hline
\end{tabular}




\begin{tabular}{|c|c|c|c|c|}
\hline SITE & COMMON NAME & SCIENTIFIC NAME & COVER & STRATUM \\
\hline 45 & Bare Ground & $N A$ & 65 & G \\
\hline 46 & Smooth Brome & Bromus inermus & 70 & $\mathrm{H}$ \\
\hline 46 & Broomsedge & Andropogon virginicus & 10 & $\mathrm{H}$ \\
\hline 46 & Late-Flowering Thoroughwort & Eupatorium serotinum & 10 & $\mathrm{H}$ \\
\hline 46 & Goldenrod & Solidago $s p$ & 5 & $\mathrm{H}$ \\
\hline 47 & Narrow-leaved Cattail & Typha angustifolia & 25 & $\mathrm{H}$ \\
\hline 47 & Slender Rush & Juncus sp. & 50 & $\mathrm{H}$ \\
\hline 47 & Fox Sedge & Carex vulpinoidea & 20 & $\mathrm{H}$ \\
\hline 47 & Broomsedge & Andropogon virginicus & 5 & $\mathrm{H}$ \\
\hline 48 & Smooth Brome & Bromus inermus & 90 & $\mathrm{H}$ \\
\hline 48 & Goldenrod & Solidago sp. & 10 & $\mathrm{H}$ \\
\hline 49 & Smooth Brome & Bromus inermus & 94 & $\mathrm{H}$ \\
\hline 49 & Common Milkweed & Asclepias syriaca & 5 & $\mathrm{H}$ \\
\hline 49 & Yarrow & Achillea millefolium & 1 & $\mathrm{H}$ \\
\hline 51 & Goldenrod & Solidago sp. & 30 & $\mathrm{H}$ \\
\hline 51 & Sericea & Sericea lespedeza & 65 & $\mathrm{H}$ \\
\hline 51 & Curled Dock & Rumex crispus & 2 & $\mathrm{H}$ \\
\hline 51 & Fox Sedge & Carex vulpinoidea & 3 & $\mathrm{H}$ \\
\hline 51 & Sycamore & Platanus occidentalis & 5 & $S$ \\
\hline 51 & American Elm & Ulmus americana & 2 & $S$ \\
\hline 53 & Oats & Avena sativa & 80 & $\mathrm{H}$ \\
\hline 53 & Ladino Clover & Trifolium repens latum & 20 & $\mathrm{H}$ \\
\hline 53 & Green Ash & Fraxinus pennsylvanica & 20 & $S$ \\
\hline 53 & Pin Oak & Quercus palustris & 5 & $\mathrm{~S}$ \\
\hline 53 & Box Elder & Acer negundo & 5 & $S$ \\
\hline 55 & Oats & Avena sativa & 50 & $\mathrm{H}$ \\
\hline 55 & Goldenrod & Solidago sp. & 50 & $\mathrm{H}$ \\
\hline 56 & Soybeans & Glycine max & 15 & $\mathrm{H}$ \\
\hline 56 & Bare Ground & $N A$ & 85 & G \\
\hline 57 & Smooth Brome & Bromus inermus & 100 & $\mathrm{H}$ \\
\hline 58 & Wild Carrot & Daucus carota & 65 & $\mathrm{H}$ \\
\hline 58 & Canada Wild Rye & Elymus canadensis & 20 & $\mathrm{H}$ \\
\hline 58 & Common Milkweed & Asclepias syriaca & 5 & $\mathrm{H}$ \\
\hline 58 & Rip-Rap & $N A$ & 10 & G \\
\hline 62 & Virginia Wild Rye & Elymus virginicus & 75 & $\mathrm{H}$ \\
\hline 62 & Black Snakeroot & Sanicula marilandica & 15 & $\mathrm{H}$ \\
\hline 62 & Blue-Eyed Mary & Collinsia verna & 5 & $\mathrm{H}$ \\
\hline 62 & Phlox & Phlox sp. & 2 & $\mathrm{H}$ \\
\hline 62 & Chickweed & Stellaria sp. & 2 & $\mathrm{H}$ \\
\hline 62 & Virginia Bluebell & Mertensia virginica & 2 & $\mathrm{H}$ \\
\hline 62 & Spring Beauty & Claytonia virginica & 1 & $\mathrm{H}$ \\
\hline 62 & American Elm & Ulmus americana & 2 & $S$ \\
\hline 62 & Sugarberry & Celtis laevigata & 5 & $S$ \\
\hline 62 & Shellbark Hickory & Carya laciniosa & 5 & $S$ \\
\hline
\end{tabular}




\begin{tabular}{|c|c|c|c|c|}
\hline SITE & COMMON NAME & SCIENTIFIC NAME & COVER & STRATUM \\
\hline 62 & Swamp White Oak & Quercus bicolor & 1 & $S$ \\
\hline 62 & Poison Ivy & Rhus radicans & 1 & V \\
\hline 62 & Sweet Cicely & Osmorhiza longistylis & 5 & $\mathrm{H}$ \\
\hline 62 & Cleavers & Galium aparine & 5 & $\mathrm{H}$ \\
\hline 62 & Coralberry & Symphoricarpos orbiculatus & 5 & S \\
\hline 63 & Fescue & Festuca arundinacea & 70 & $\mathrm{H}$ \\
\hline 63 & Smooth Brome & Bromus inermus & 20 & $\mathrm{H}$ \\
\hline 63 & Residue Covered Ground & $N A$ & 10 & G \\
\hline 64 & Red Clover & Trifolium pratense & 65 & $\mathrm{H}$ \\
\hline 64 & Fescue & Festuca arundinacea & 25 & $\mathrm{H}$ \\
\hline 64 & Residue Covered Ground & $N A$ & 10 & $G$ \\
\hline 67 & Smooth Brome & Bromus inermus & 85 & $\mathrm{H}$ \\
\hline 67 & Musk Thistle & Carduus nutans & 5 & $\mathrm{H}$ \\
\hline 67 & Sweet Clover & Melilotus sp. & 10 & $\mathrm{H}$ \\
\hline 68 & Fescue & Festuca arundinacea & 95 & $\mathrm{H}$ \\
\hline 68 & Goldenrod & Solidago sp. & 5 & $\mathrm{H}$ \\
\hline 69 & Smooth Brome & Bromus inermus & 95 & $\mathrm{H}$ \\
\hline 69 & Musk Thistle & Carduus nutans & 5 & $\mathrm{H}$ \\
\hline 69 & Honey Locust & Gleditsia triacanthos & 85 & $S$ \\
\hline 72 & Oats & Avena sativa & 30 & $\mathrm{H}$ \\
\hline 72 & Goldenrod & Solidago sp. & 70 & $\mathrm{H}$ \\
\hline 72 & Green Ash & Fraxinus pennsylvanica & 5 & S \\
\hline 72 & American Elm & Ulmus americana & 2 & s \\
\hline 72 & Honey Locust & Gleditsia triacanthos & 5 & s \\
\hline 73 & Fescue & Festuca arundinacea & 100 & $\mathrm{H}$ \\
\hline 74 & Fescue & Festuca arundinacea & 88 & $\mathrm{H}$ \\
\hline 74 & Timothy & Phleum pratense & 2 & $\mathrm{H}$ \\
\hline 74 & Residue Covered Ground & $N A$ & 10 & G \\
\hline 75 & Fescue & Festuca arundinacea & 80 & $\mathrm{H}$ \\
\hline 75 & Red Clover & Trifolium pratense & 10 & $\mathrm{H}$ \\
\hline 75 & Smooth Brome & Bromus inermus & 10 & $\mathrm{H}$ \\
\hline 77 & Orchardgrass & Dactylis glomerata & 45 & $\mathrm{H}$ \\
\hline 77 & Goldenrod & Solidago sp. & 30 & $\mathrm{H}$ \\
\hline 77 & Smooth Brome & Bromus inermus & 10 & $\mathrm{H}$ \\
\hline 77 & Residue Covered Ground & $N A$ & 15 & G \\
\hline 77 & Eastern Red Cedar & Juniperus virginiana & 5 & $S$ \\
\hline 77 & Dogwood Shrub & Cornus sp. & 5 & $S$ \\
\hline 78 & Ag. Field & $N A$ & 100 & NA \\
\hline 79 & Ag. Field & $N A$ & 100 & NA \\
\hline 82 & Goldenrod & Solidago sp. & 75 & $\mathrm{H}$ \\
\hline 82 & Red Clover & Trifolium pratense & 20 & $\mathrm{H}$ \\
\hline 82 & Sweet White Clover & Melilotus alba & 5 & $\mathrm{H}$ \\
\hline 84 & Green Ash & Fraxinus pennsylvanica & 20 & $S$ \\
\hline 84 & Box Elder & Acer negundo & 20 & $S$ \\
\hline
\end{tabular}


SITE COMMON NAME

\section{4}

\section{4}

84

84

84

84

84

84

84

84

84

84

84

85
Silver Maple

American EIm

Prickly Brambles

Japanese Honeysuckle

Grapevine

Trumpet Creeper

Fox Sedge

Sedge

Poison Ivy

Common Ragweed

Canada Wild Rye

Rush

Smartweed

Fescue

Curled Dock

Sweet Yellow Clover

Smooth Brome

Fescue

Smooth Brome

Residue Covered Ground

Fescue

White Clover

Residue Covered Ground

Fescue

Orchardgrass

Smooth Brome

Residue Covered Ground

Smooth Brome

Orchardgrass

Fescue

Goldenrod

American Elm

Dogwood Shrub

Ag. Field

Ag. Field

Fescue

Goldenrod

Orchardgrass

Sumac

Willow

Grapevine

Black Snakeroot

Virginia Wild Rye

Virginia Creeper
SCIENTIFIC NAME

COVER STRATUM

Acer saccharinum

20 S

Ulmus americana

Rubus sp.

Lonicera japonica

Vitis sp.

Campsis radicans

Carex vulpinoidea

Carex sp.

Rhus radicans

Ambrosia artemisiifolia

Elymus canadensis

Juncus sp.

Polygonum pensylvanicum

Festuca arundinacea

Rumex crispus

Melilotus officinalis

Bromus inermus

Festuca arundinacea

Bromus inermus

NA

Festuca arundinacea

Trifolium repens

NA

Festuca arundinacea

Dactylis glomerata

Bromus inermus

NA

Bromus inermus

Dactylis glomerata

Festuca arundinacea

Solidago $s p$.

Ulmus americana

Cornus sp.

NA

NA

Festuca arundinacea

Solidago $s p$.

Dactylis glomerata

Rhus sp.

Salix $s p$.

Vitis sp.

Sanicula marilandica

Elymus virginicus

Parthenosensis quinqifolia

20 S

$10 \mathrm{~S}$

$30 \mathrm{~V}$

$10 \mathrm{~V}$

$20 \quad \mathrm{H}$

10

20

10

$S$

S

V

H

$\mathrm{H}$

$\mathrm{H}$

$\mathrm{H}$

$\mathrm{H}$

$\mathrm{H}$

$\mathrm{H}$

$\mathrm{H}$

$\mathrm{H}$

$\mathrm{H}$

$\mathrm{H}$

$20 \mathrm{H}$

$5 \quad \mathrm{H}$

$80 \mathrm{H}$

$10 \mathrm{H}$

$10 \quad G$

$60 \mathrm{H}$

$10 \mathrm{H}$

30 G

$60 \mathrm{H}$

$5 \quad \mathrm{H}$

$10 \quad \mathrm{H}$

$25 \quad G$

$70 \quad \mathrm{H}$

$10 \mathrm{H}$

$10 \mathrm{H}$

$10 \mathrm{H}$

$5 \mathrm{~S}$

10 S

100 NA

100 NA

$80 \mathrm{H}$

$10 \mathrm{H}$

$10 \mathrm{H}$

$20 \mathrm{~S}$

$60 \quad \mathrm{~T}$

$5 \mathrm{~V}$

$40 \quad \mathrm{H}$

$10 \mathrm{H}$

$5 \quad \mathrm{H} / \mathrm{V}$




\begin{tabular}{|c|c|c|c|c|}
\hline SITE & COMMON NAME & SCIENTIFIC NAME & COVER & STRATUM \\
\hline 93 & Yellow Violet & Viola pensylvanica & 5 & $\mathrm{H}$ \\
\hline 93 & Phlox & Phlox sp. & 2 & $\mathrm{H}$ \\
\hline 93 & Jewelweed & Impatiens capensis & 2 & $\mathrm{H}$ \\
\hline 93 & Pennsylvania Bittercress & Cardamine pensylvanica & 1 & $\mathrm{H}$ \\
\hline 93 & Catbrier & Smilax sp. & 1 & V \\
\hline 93 & Trumpet Creeper & Campsis radicans & 10 & V \\
\hline 93 & Shellbark Hickory & Carya laciniosa & 10 & $\mathrm{~S}$ \\
\hline 93 & Sugarberry & Celtis laevigata & 10 & $\mathrm{~S}$ \\
\hline 93 & May Apple & Podophyllum peltatum & 2 & $\mathrm{H}$ \\
\hline 93 & Red Trillium & Trillium erectum & 2 & $\mathrm{H}$ \\
\hline 93 & Solomon's Seal & Smilacina sp. & 1 & $\mathrm{H}$ \\
\hline 93 & Poison Ivy & Rhus radicans & 5 & $\mathrm{H}$ \\
\hline 93 & Honewort & Cryptotaenia canadensis & 2 & $\mathrm{H}$ \\
\hline 93 & Grapevine & Vitis sp. & 1 & V \\
\hline 93 & Trout Lily & Erythronium americanum & 1 & $\mathrm{H}$ \\
\hline 95 & Fescue & Festuca arundinacea & 100 & $\mathrm{H}$ \\
\hline 96 & Goldenrod & Solidago sp. & 20 & $\mathrm{H}$ \\
\hline 96 & Sweet Yellow Clover & Melilotus officinalis & 20 & $\mathrm{H}$ \\
\hline 96 & Orchardgrass & Dactylis glomerata & 40 & $\mathrm{H}$ \\
\hline 96 & Smooth Brome & Bromus inermus & 20 & $\mathrm{H}$ \\
\hline 97 & Fescue & Festuca arundinacea & 100 & $\mathrm{H}$ \\
\hline 101 & Fescue & Festuca arundinacea & 20 & $\mathrm{H}$ \\
\hline 101 & Orchardgrass & Dactylis glomerata & 60 & $\mathrm{H}$ \\
\hline 101 & Bare Ground & $N A$ & 20 & G \\
\hline 102 & Ag. Field & $N A$ & 100 & NA \\
\hline 103 & Ag. Field & $N A$ & 100 & NA \\
\hline 104 & Sweet Cicely & Osmorhiza longistylis & 10 & $\mathrm{H}$ \\
\hline 104 & Virginia Wild Rye & Elymus virginicus & 30 & $\mathrm{H}$ \\
\hline 104 & Cleavers & Galium aparine & 10 & $\mathrm{H}$ \\
\hline 104 & Black Snakeroot & Sanicula marilandica & 5 & $\mathrm{H}$ \\
\hline 104 & Honewort & Cryptotaenia canadensis & 5 & $\mathrm{H}$ \\
\hline 104 & Stinging Nettle & Urtica dioica & 1 & $\mathrm{H}$ \\
\hline 104 & Trumpet Creeper & Campsis radicans & 5 & V \\
\hline 104 & Bitternut Hickory & Carya cordiformis & 2 & $S$ \\
\hline 104 & American Elm & Ulmus americana & 5 & $\mathrm{~S}$ \\
\hline 104 & Silver Maple & Acer saccharinum & 5 & $\mathrm{~S}$ \\
\hline 104 & Box Elder & Acer negundo & 5 & $S$ \\
\hline 106 & Fescue & Festuca arundinacea & 75 & $\mathrm{H}$ \\
\hline 106 & Fox Sedge & Carex vulpinoidea & 20 & $\mathrm{H}$ \\
\hline 106 & Rush & Juncus sp. & 5 & $\mathrm{H}$ \\
\hline 107 & Fescue & Festuca arundinacea & 90 & $\mathrm{H}$ \\
\hline 107 & Smooth Brome & Bromus inermus & 10 & $\mathrm{H}$ \\
\hline 108 & Fescue & Festuca arundinacea & 50 & $\mathrm{H}$ \\
\hline 108 & Orchardgrass & Dactylis glomerata & 30 & $\mathrm{H}$ \\
\hline
\end{tabular}


SITE 108

110

110

111

111

112

112

113

113

113

113

114

115

116

116

116

116

116

116

116

\section{6}

116
COMMON NAME

Smooth Brome

Fescue

Residue Covered Ground

Smooth Brome

Residue Covered Ground

Smooth Brome

Orchardgrass

Smooth Brome

Goldenrod

Prickly Brambles

Residue Covered Ground

Ag. Field

Ag. Field

Black Snakeroot

Stinging Nettle

Virginia Wild Rye

Buttercup

Jewelweed

Catbrier

Box Elder

Pin Oak

Spring Beauty

Yellow Violet

Poison Ivy

Bedstraw

Sugarberry

Multiflora Rose

Chickweed

Sweet Cicely

Smooth Brome

Fescue

White Clover

Late-Flowering Thoroughwort

Curled Dock

Fescue

Fescue

Timothy

Fox Sedge

Smooth Brome

Sweet Yellow Clover

Residue Covered Ground

Fescue

Goldenrod

Residue Covered Ground
SCIENTIFIC NAME

COVER STRATUM

Bromus inermus

$20 \mathrm{H}$

Festuca arundinacea

$80 \quad \mathrm{H}$

NA

$20 \quad \mathrm{G}$

Bromus inermus

$90 \quad \mathrm{H}$

NA

Bromus inermus

$10 \quad$ G

Dactylis glomerata

$70 \quad \mathrm{H}$

Bromus inermus

Solidago sp.

Rubus sp.

NA

$N A$

NA

Sanicula marilandica

Urtica dioica

Elymus virginicus

Ranunculus $s p$.

Impatiens capensis

Smilax sp.

Acer negundo

Quercus palustris

Claytonia virginica

Viola pensylvanica

Rhus radicans

Galium sp.

Celtis laevigata

Rosa multiflora

Stellaria sp.

Osmorhiza longistylis

Bromus inermus

Festuca arundinacea

Trifolium repens

Eupatorium serotinum

Rumex crispus

Festuca arundinacea

Festuca arundinacea

Phleum pratense

Carex vulpinoidea

Bromus inermus

Melilotus officinalis

NA

Festuca arundinacea

Solidago $s p$.

NA

$30 \quad \mathrm{H}$

$70 \quad \mathrm{H}$

$20 \quad \mathrm{H}$

$1 \mathrm{H}$

$9 \quad \mathrm{G}$

100 NA

100 NA

$30 \quad \mathrm{H}$

$20 \quad \mathrm{H}$

$5 \quad \mathrm{H}$

$2 \mathrm{H}$

$10 \mathrm{H}$

$2 \mathrm{H}$

$2 \mathrm{~S}$

$2 \mathrm{~S}$

$10 \mathrm{H}$

$1 \mathrm{H}$

$5 \quad \mathrm{H}$

$10 \mathrm{H}$

2 S

$1 \mathrm{~S}$

$2 \mathrm{H}$

$10 \mathrm{H}$

$10 \mathrm{H}$

$70 \quad \mathrm{H}$

$10 \quad \mathrm{H}$

$5 \quad \mathrm{H}$

$5 \quad \mathrm{H}$

$100 \quad \mathrm{H}$

$90 \quad \mathrm{H}$

$5 \quad \mathrm{H}$

$5 \quad \mathrm{H}$

$85 \mathrm{H}$

$5 \quad \mathrm{H}$

10 G

$30 \quad \mathrm{H}$

$10 \quad \mathrm{G}$

$60 \quad \mathrm{H}$




\begin{tabular}{|c|c|c|c|c|}
\hline SITE & COMMON NAME & SCIENTIFIC NAME & COVER & STRATUM \\
\hline 123 & Fescue & Festuca arundinacea & 60 & $\mathrm{H}$ \\
\hline 123 & Goldenrod & Solidago sp. & 30 & $\mathrm{H}$ \\
\hline 123 & Residue Covered Ground & $N A$ & 10 & G \\
\hline 124 & Fescue & Festuca arundinacea & 85 & $\mathrm{H}$ \\
\hline 124 & Broomsedge & Andropogon virginicus & 5 & $\mathrm{H}$ \\
\hline 124 & Goldenrod & Solidago sp. & 10 & $\mathrm{H}$ \\
\hline 125 & Sedge & Carex sp. & 20 & $\mathrm{H}$ \\
\hline 125 & Horseweed & Erigeron canadensis & 5 & $\mathrm{H}$ \\
\hline 125 & Pigweed & Amaranthus sp. & 10 & $\mathrm{H}$ \\
\hline 125 & Wild Onion & Allium canadense & 2 & $\mathrm{H}$ \\
\hline 125 & Winter Wheat & Triticum aestivum & 2 & $\mathrm{H}$ \\
\hline \multirow[t]{2}{*}{125} & Late-Flowering Thoroughwort & Eupatorium serotinum & 2 & $\mathrm{H}$ \\
\hline & Residue Covered Ground and Bare & & & \\
\hline 125 & Ground & $N A$ & 59 & G \\
\hline 126 & Sedge & Carex sp. & 5 & $\mathrm{H}$ \\
\hline 126 & Horseweed & Erigeron canadensis & 5 & $\mathrm{H}$ \\
\hline 126 & Butterweed & Senecio aureus & 2 & $\mathrm{H}$ \\
\hline 126 & Pigweed & Amaranthus sp. & 5 & $\mathrm{H}$ \\
\hline \multirow[t]{2}{*}{126} & Buttercup & Ranunculus sp. & 2 & $\mathrm{H}$ \\
\hline & Residue Covered Ground and Bare & & & \\
\hline 126 & Ground & $N A$ & 81 & G \\
\hline 127 & Chickweed & Stellaria sp. & 70 & $\mathrm{H}$ \\
\hline 127 & Horseweed & Erigeron canadensis & 5 & $\mathrm{H}$ \\
\hline 127 & Pokeweed & Phytolacca americana & 5 & $\mathrm{H}$ \\
\hline 127 & Jimsonweed & Datura stramonium & 2 & $\mathrm{H}$ \\
\hline 127 & Pigweed & Amaranthus sp. & 5 & $\mathrm{H}$ \\
\hline 127 & Residue Covered Ground & $N A$ & 13 & $\mathrm{H}$ \\
\hline 128 & Virginia Wild Rye & Elymus virginicus & 85 & $\mathrm{H}$ \\
\hline 128 & Honewort & Cryptotaenia canadensis & 5 & $\mathrm{H}$ \\
\hline 128 & Black Snakeroot & Sanicula marilandica & 10 & $\mathrm{H}$ \\
\hline 128 & Stinging Nettle & Urtica dioica & 10 & $\mathrm{H}$ \\
\hline 128 & Phlox & Phlox sp. & 2 & $\mathrm{H}$ \\
\hline 128 & Grapevine & Vitis sp. & 2 & V \\
\hline 128 & American Elm & Ulmus americana & 2 & S \\
\hline 128 & Sugarberry & Celtis laevigata & 2 & $S$ \\
\hline 128 & Virginia Creeper & Parthenosensis quinqifolia & 1 & V \\
\hline 128 & Jewelweed & Impatiens capensis & 2 & $\mathrm{H}$ \\
\hline 128 & Yellow Violet & Viola pensylvanica & 2 & $\mathrm{H}$ \\
\hline 128 & Spring Beauty & Claytonia virginica & 2 & $\mathrm{H}$ \\
\hline 128 & Blue-Eyed Mary & Collinsia verna & 1 & $\mathrm{H}$ \\
\hline 129 & Fescue & Festuca arundinacea & 60 & $\mathrm{H}$ \\
\hline 129 & Goldenrod & Solidago sp. & 10 & $\mathrm{H}$ \\
\hline 129 & Bare Ground & $N A$ & 30 & $\mathrm{H}$ \\
\hline 130 & Smooth Brome & Bromus inermus & 70 & $\mathrm{H}$ \\
\hline 130 & Residue Covered Ground & $N A$ & 30 & $\mathrm{H}$ \\
\hline
\end{tabular}


SITE

131

131

133

133

133

133

133

133

136

136

136

137

137

137

137

137

137

138

138

138

138

138

138

138

138

138

139

139

139

140

140

140

141

142

142

143

143

144

144

144

144

144

146
COMMON NAME

Smooth Brome

Residue Covered Ground and Bare

Ground

Fescue

Goldenrod

Ladino Clover

Smooth Brome

Curled Dock

Bare Ground

Fescue

Sweet Yellow Clover

Red Clover

Sedge

Pigweed

Horseweed

Butterweed

Aster

Residue Covered Ground and Bare

Ground

Virginia Wild Rye

Black Snakeroot

Cleavers

Yellow Violet

Jewelweed

Stinging Nettle

Sweet William

Hackberry

Box Elder

Fescue

Sweet Yellow Clover

Bare Ground

Smooth Brome

Aster

Residue Covered Ground

Smooth Brome

Smooth Brome

Orchardgrass

Smooth Brome

Orchardgrass

Kentucky Bluegrass

Fescue

Fox Sedge

Red Clover

Residue Covered Ground

Fescue
SCIENTIFIC NAME

Bromus inermus

COVER STRATUM

$60 \mathrm{H}$

NA

Festuca arundinacea

Solidago sp.

Trifolium repens latum

Bromus inermus

Rumex crispus

$N A$

Festuca arundinacea

Melilotus officinalis

Trifolium pratense

Carex sp.

Amaranthus sp.

Erigeron canadensis

Senecio aureus

Aster sp.

$N A$

Elymus virginicus

Sanicula marilandica

Galium aparine

Viola pensylvanica

Impatiens capensis

Urtica dioica

Phlox maculata

Celtis occidentalis

Acer negundo

Festuca arundinacea

Melilotus officinalis

$N A$

Bromus inermus

Aster sp.

NA

Bromus inermus

Bromus inermus

Dactylis glomerata

Bromus inermus

Dactylis glomerata

Poa pratensis

Festuca arundinacea

Carex vulpinoidea

Trifolium pratense

$N A$

Festuca arundinacea
$40 \quad \mathrm{H}$

$60 \quad \mathrm{H}$

\section{H}

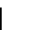

H

$10 \mathrm{H}$

$5 \quad \mathrm{H}$

$2 \quad \mathrm{H}$

13 G

$90 \quad \mathrm{H}$

$5 \mathrm{H}$

$5 \quad \mathrm{H}$

$10 \quad \mathrm{H}$

$5 \mathrm{H}$

$5 \quad \mathrm{H}$

$5 \quad \mathrm{H}$

$5 \mathrm{H}$

$70 \quad$ G

$70 \quad \mathrm{H}$

$20 \quad \mathrm{H}$

$5 \quad \mathrm{H}$

$2 \mathrm{H}$

$5 \quad \mathrm{H}$

$5 \quad \mathrm{H}$

$2 \mathrm{H}$

$5 \mathrm{~S}$

$2 S$

$50 \quad \mathrm{H}$

$40 \quad \mathrm{H}$

$10 \quad$ G

$80 \quad \mathrm{H}$

$1 \mathrm{H}$

19 G

$100 \quad \mathrm{H}$

$70 \quad \mathrm{H}$

$30 \quad \mathrm{H}$

$80 \mathrm{H}$

$20 \mathrm{H}$

$60 \mathrm{H}$

$10 \mathrm{H}$

$5 \mathrm{H}$

$1 \mathrm{H}$

$24 \quad G$

$95 \mathrm{H}$

$10 \quad \mathrm{H}$




\begin{tabular}{|c|c|c|c|c|}
\hline SITE & COMMON NAME & SCIENTIFIC NAME & COVER & STRATUM \\
\hline 146 & Sweet Yellow Clover & Melilotus officinalis & 5 & $\mathrm{H}$ \\
\hline 148 & Smooth Brome & Bromus inermus & 90 & $\mathrm{H}$ \\
\hline 148 & Residue Covered Ground & $N A$ & 10 & G \\
\hline 149 & Smooth Brome & Bromus inermus & 65 & $\mathrm{H}$ \\
\hline 149 & Goldenrod & Solidago sp. & 10 & $\mathrm{H}$ \\
\hline 149 & Red Clover & Trifolium pratense & 5 & $\mathrm{H}$ \\
\hline 149 & Residue Covered Ground & $N A$ & 20 & G \\
\hline 150 & Phragmites & Phragmites australis & 100 & $\mathrm{H}$ \\
\hline 151 & Smooth Brome & Bromus inermus & 50 & $\mathrm{H}$ \\
\hline 151 & Orchardgrass & Dactylis glomerata & 50 & $\mathrm{H}$ \\
\hline 152 & Smooth Brome & Bromus inermus & 75 & $\mathrm{H}$ \\
\hline 152 & Orchardgrass & Dactylis glomerata & 10 & $\mathrm{H}$ \\
\hline 152 & White Clover & Trifolium repens & 5 & $\mathrm{H}$ \\
\hline 152 & Residue Covered Ground & $N A$ & 10 & G \\
\hline 153 & Orchardgrass & Dactylis glomerata & 90 & $\mathrm{H}$ \\
\hline 153 & Smooth Brome & Bromus inermus & 10 & $\mathrm{H}$ \\
\hline 154 & Smooth Brome & Bromus inermus & 50 & $\mathrm{H}$ \\
\hline 154 & Fescue & Festuca arundinacea & 25 & $\mathrm{H}$ \\
\hline 154 & Green Bulrush & Scirpus atrovirens & 10 & $\mathrm{H}$ \\
\hline 154 & Wet bare ground & $N A$ & 15 & G \\
\hline 155 & Smooth Brome & Bromus inermus & 65 & $\mathrm{H}$ \\
\hline 155 & Orchardgrass & Dactylis glomerata & 10 & $\mathrm{H}$ \\
\hline 155 & Alfalfa & Medicago sativa & 15 & $\mathrm{H}$ \\
\hline 155 & Residue Covered Ground & $N A$ & 10 & G \\
\hline 156 & Fescue & Festuca arundinacea & 45 & $\mathrm{H}$ \\
\hline 156 & Sweet Yellow Clover & Melilotus officinalis & 15 & $\mathrm{H}$ \\
\hline 156 & Red Clover & Trifolium pratense & 30 & $\mathrm{H}$ \\
\hline 156 & Goldenrod & Solidago sp. & 10 & $\mathrm{H}$ \\
\hline 157 & Sweet Yellow Clover & Melilotus officinalis & 30 & $\mathrm{H}$ \\
\hline 157 & Fescue & Festuca arundinacea & 5 & $\mathrm{H}$ \\
\hline 157 & Goldenrod & Solidago sp. & 5 & $\mathrm{H}$ \\
\hline 157 & Bare Ground & $N A$ & 60 & G \\
\hline 158 & Barley & Hordeum vulgare & 5 & $\mathrm{H}$ \\
\hline 158 & Gravel & $N A$ & 95 & G \\
\hline 159 & Sweet Yellow Clover & Melilotus officinalis & 75 & $\mathrm{H}$ \\
\hline 159 & Red Clover & Trifolium pratense & 5 & $\mathrm{H}$ \\
\hline 159 & Smooth Brome & Bromus inermus & 10 & $\mathrm{H}$ \\
\hline 159 & Fescue & Festuca arundinacea & 5 & $\mathrm{H}$ \\
\hline 159 & Gravel & $N A$ & 5 & G \\
\hline 160 & Smooth Brome & Bromus inermus & 85 & $\mathrm{H}$ \\
\hline 160 & Residue Covered Ground & $N A$ & 15 & G \\
\hline 161 & Smooth Brome & Bromus inermus & 80 & $\mathrm{H}$ \\
\hline 161 & Orchardgrass & Dactylis glomerata & 20 & $\mathrm{H}$ \\
\hline 162 & Smooth Brome & Bromus inermus & 40 & $\mathrm{H}$ \\
\hline
\end{tabular}




\begin{tabular}{|c|c|c|c|c|}
\hline SITE & COMMON NAME & SCIENTIFIC NAME & COVER & STRATUM \\
\hline 162 & Broomsedge & Andropogon virginicus & 20 & $\mathrm{H}$ \\
\hline 162 & Goldenrod & Solidago sp. & 35 & $\mathrm{H}$ \\
\hline 162 & Panicgrass & Dicanthelium sp. & 5 & $\mathrm{H}$ \\
\hline 162 & Prickly Brambles & Rubus sp. & 2 & $\mathrm{H}$ \\
\hline 162 & Trumpet Creeper & Campsis radicans & 2 & $\mathrm{H}$ \\
\hline 162 & Sedge & Carex sp. & 1 & $\mathrm{H}$ \\
\hline 163 & Broomsedge & Andropogon virginicus & 30 & $\mathrm{H}$ \\
\hline 163 & Smooth Brome & Bromus inermus & 60 & $\mathrm{H}$ \\
\hline 163 & Common Milkweed & Asclepias syriaca & 2 & $\mathrm{H}$ \\
\hline 163 & Goldenrod & Solidago sp. & 3 & $\mathrm{H}$ \\
\hline 163 & Musk Thistle & Carduus nutans & 5 & $\mathrm{H}$ \\
\hline 164 & Smooth Brome & Bromus inermus & 80 & $\mathrm{H}$ \\
\hline 164 & Residue Covered Ground & $N A$ & 20 & G \\
\hline 165 & Orchardgrass & Dactylis glomerata & 35 & $\mathrm{H}$ \\
\hline 165 & Fescue & Festuca arundinacea & 20 & $\mathrm{H}$ \\
\hline 165 & Ladino Clover & Trifolium repens latum & 10 & $\mathrm{H}$ \\
\hline 165 & Red Clover & Trifolium pratense & 5 & $\mathrm{H}$ \\
\hline 165 & Aster & Aster sp. & 2 & $\mathrm{H}$ \\
\hline 165 & Bare Ground & $N A$ & 28 & G \\
\hline 166 & Stinging Nettle & Urtica dioica & 25 & $\mathrm{H}$ \\
\hline 166 & Black Snakeroot & Sanicula marilandica & 20 & $\mathrm{H}$ \\
\hline 166 & May Apple & Podophyllum peltatum & 10 & $\mathrm{H}$ \\
\hline 166 & Hackberry & Celtis occidentalis & 5 & $S$ \\
\hline 166 & Grapevine & Vitis $s p$ & 1 & V \\
\hline 166 & Poison Ivy & Rhus radicans & 2 & $\mathrm{H}$ \\
\hline 166 & Stout Woodreed & Cinna arundinacea & 2 & $\mathrm{H}$ \\
\hline 166 & Bare Ground & $N A$ & 35 & G \\
\hline 167 & Virginia Wild Rye & Elymus virginicus & 10 & $\mathrm{H}$ \\
\hline 167 & Stinging Nettle & Urtica dioica & 10 & $\mathrm{H}$ \\
\hline 167 & Cleavers & Galium aparine & 5 & $\mathrm{H}$ \\
\hline 167 & Moneywort & Lysimachia nummularia & 5 & $\mathrm{H}$ \\
\hline 167 & Box Elder & Acer negundo & 2 & $\mathrm{~S}$ \\
\hline 167 & Silver Maple & Acer saccharinum & 5 & $S$ \\
\hline 167 & Poison Ivy & Rhus radicans & 2 & $\mathrm{H}$ \\
\hline 167 & Bare Ground & $N A$ & 61 & G \\
\hline 168 & Virginia Wild Rye & Elymus virginicus & 20 & $\mathrm{H}$ \\
\hline 168 & Chickweed & Stellaria sp. & 20 & $\mathrm{H}$ \\
\hline 168 & Henbit & Lamium amplexicaule & 15 & $\mathrm{H}$ \\
\hline 168 & Sweet William & Phlox maculata & 5 & $\mathrm{H}$ \\
\hline 168 & Tumble mustard & Sisymbrium altissimum & 2 & $\mathrm{H}$ \\
\hline 168 & Box Elder & Acer negundo & 5 & $S$ \\
\hline 168 & River Birch & Betula nigra & 5 & $S$ \\
\hline 168 & Poison Ivy & Rhus radicans & 2 & V \\
\hline 168 & Green Ash & Fraxinus pennsylvanica & 2 & $S$ \\
\hline
\end{tabular}




\begin{tabular}{|c|c|c|c|c|}
\hline SITE & COMMON NAME & SCIENTIFIC NAME & COVER & STRATUM \\
\hline 168 & Hackberry & Celtis occidentalis & 1 & $S$ \\
\hline 168 & Bare Ground & $N A$ & 23 & G \\
\hline 169 & Wheat & Triticum aestivum & 7 & $\mathrm{H}$ \\
\hline 169 & Goldenrod & Solidago sp. & 5 & $\mathrm{H}$ \\
\hline 169 & Oats & Avena sativa & 1 & $\mathrm{H}$ \\
\hline 169 & Fescue & Festuca arundinacea & 2 & $\mathrm{H}$ \\
\hline 169 & Bare Ground & $N A$ & 85 & G \\
\hline 170 & Honey Locust & Gleditsia triacanthos & 60 & $\mathrm{H} / \mathrm{S}$ \\
\hline 170 & American Elm & Ulmus americana & 35 & $\mathrm{H} / \mathrm{S}$ \\
\hline 170 & Virginia Wild Rye & Elymus virginicus & 40 & $\mathrm{H}$ \\
\hline 170 & Pokeweed & Phytolacca americana & 5 & $\mathrm{H}$ \\
\hline 170 & Poison Ivy & Rhus radicans & 10 & $\mathrm{H}$ \\
\hline 170 & Virginia Creeper & Parthenosensis quinqifolia & 5 & $\mathrm{H}$ \\
\hline 170 & Jewelweed & Impatiens capensis & 5 & $\mathrm{H}$ \\
\hline 171 & Smooth Brome & Bromus inermus & 90 & $\mathrm{H}$ \\
\hline 171 & Fescue & Festuca arundinacea & 5 & $\mathrm{H}$ \\
\hline 171 & Aster & Aster sp. & 5 & $\mathrm{H}$ \\
\hline 172 & Smooth Brome & Bromus inermus & 60 & $\mathrm{H}$ \\
\hline 172 & Rush & Juncus sp. & 5 & $\mathrm{H}$ \\
\hline 172 & Orchardgrass & Dactylis glomerata & 2 & $\mathrm{H}$ \\
\hline 172 & Blue Vervain & Verbena hastata & 5 & $\mathrm{H}$ \\
\hline \multirow[t]{2}{*}{172} & Fescue & Festuca arundinacea & 3 & $\mathrm{H}$ \\
\hline & Residue Covered Ground and Bare & & & \\
\hline 172 & Ground & $N A$ & 25 & G \\
\hline 173 & Smooth Brome & Bromus inermus & 75 & $\mathrm{H}$ \\
\hline 173 & Orchardgrass & Dactylis glomerata & 2 & $\mathrm{H}$ \\
\hline 173 & Residue Covered Ground & $N A$ & 23 & G \\
\hline 174 & Smooth Brome & Bromus inermus & 65 & $\mathrm{H}$ \\
\hline \multirow[t]{2}{*}{174} & Aster & Aster sp. & 5 & $\mathrm{H}$ \\
\hline & Residue Covered Ground and Bare & & & \\
\hline 174 & Ground & $N A$ & 30 & G \\
\hline 175 & Smooth Brome & Bromus inermus & 85 & $\mathrm{H}$ \\
\hline 175 & Orchardgrass & Dactylis glomerata & 5 & $\mathrm{H}$ \\
\hline 175 & Trumpet Creeper & Campsis radicans & 10 & $\mathrm{H}$ \\
\hline 176 & Smooth Brome & Bromus inermus & 70 & $\mathrm{H}$ \\
\hline 176 & Orchardgrass & Dactylis glomerata & 10 & $\mathrm{H}$ \\
\hline 176 & Oats & Avena sativa & 10 & $\mathrm{H}$ \\
\hline 176 & Residue Covered Ground & $N A$ & 10 & G \\
\hline 177 & Smooth Brome & Bromus inermus & 55 & $\mathrm{H}$ \\
\hline 177 & Residue Covered Ground & $N A$ & 45 & G \\
\hline 178 & Fescue & Festuca arundinacea & 70 & $\mathrm{H}$ \\
\hline 178 & Alfalfa & Medicago sativa & 15 & $\mathrm{H}$ \\
\hline 178 & Orchardgrass & Dactylis glomerata & 5 & $\mathrm{H}$ \\
\hline 178 & Aster & Aster sp. & 2 & $\mathrm{H}$ \\
\hline 178 & Bare Ground & $N A$ & 8 & G \\
\hline
\end{tabular}


SITE

179

\section{0}

180

180

181

183

183

183

183

183

183

184

185

185

185

185

185

186

186

187

187

187

187

188

188

188

189

189

189

189

189

189

190

190

191

191

191

195

195

195

195

196

196
COMMON NAME

Winter Wheat

Goldenrod

Wheat

Wild Carrot

Fescue

White Clover

Sweet Yellow Clover

Aster

Smooth Brome

Goldenrod

Common Milkweed

Smooth Brome

Smooth Brome

Rush

Late-Flowering Thouroughwort

Chickweed

Residue Covered Ground and Bare

Ground

Smooth Brome

Residue Covered Ground

Fescue

Orchardgrass

Residue Covered Ground

Trumpet Creeper

Goldenrod

Smooth Brome

Honey Locust

Fescue

Smooth Brome

Orchardgrass

Late-Flowering Thoroughwort

Rush

Residue Covered Ground

Smooth Brome

Residue Covered Ground

Fescue

Kentucky Bluegrass

Residue Covered Ground and Bare Ground

Perennial Rye

Chickweed

Downy Brome

Bare Ground

Red Clover

Smooth Brome
SCIENTIFIC NAME

COVER STRATUM

Triticum aestivum

$100 \quad \mathrm{H}$

Solidago sp.

$40 \quad \mathrm{H}$

Triticum aestivum

$50 \quad \mathrm{H}$

Daucus carota

$10 \quad \mathrm{H}$

Festuca arundinacea

$100 \quad \mathrm{H}$

Trifolium repens

Melilotus officinalis

$75 \quad \mathrm{H}$

Aster sp.

Bromus inermus

Solidago sp.

Asclepias syriaca

Bromus inermus

Bromus inermus

Juncus sp.

Eupatorium serotinum

Stellaria sp.

(

(n)

H

H

H

H

$8 \quad \mathrm{H}$

$5 \quad \mathrm{H}$

$5 \quad \mathrm{H}$

$5 \mathrm{H}$

$2 \mathrm{H}$

$100 \quad \mathrm{H}$

$70 \quad \mathrm{H}$

$1 \mathrm{H}$

$2 \mathrm{H}$

$2 \mathrm{H}$

NA

$25 \quad$ G

Bromus inermus $\quad 90 \quad \mathrm{H}$

$\begin{array}{lll}N A & 10 \quad \mathrm{G}\end{array}$

Festuca arundinacea $\quad 40 \quad \mathrm{H}$

Dactylis glomerata $\quad 40 \quad \mathrm{H}$

NA $15 \quad \mathrm{H}$

Campsis radicans $\quad 5 \quad \mathrm{H}$

$\begin{array}{llll}\text { Solidago sp. } & 30 & \text { H }\end{array}$

Bromus inermus $\quad 70 \quad \mathrm{H}$

Gleditsia triacanthos $\quad 70 \quad \mathrm{~S}$

Festuca arundinacea $\quad 60 \quad \mathrm{H}$

Bromus inermus $\quad 10 \quad \mathrm{H}$

Dactylis glomerata $\quad 5 \quad \mathrm{H}$

Eupatorium serotinum $\quad 5 \quad \mathrm{H}$

Juncus sp. $10 \quad \mathrm{H}$

NA $10 \quad$ G

Bromus inermus $\quad 85 \quad \mathrm{H}$

$\begin{array}{lll}N A & 15 & G\end{array}$

Festuca arundinacea $\quad 80 \quad \mathrm{H}$

$\begin{array}{lll}\text { Poa pratensis } & 5 & \mathrm{H}\end{array}$

$\begin{array}{lll}N A & 15 \quad \mathrm{G}\end{array}$

Lolium perenne $\quad 50 \quad \mathrm{H}$

$\begin{array}{lll}\text { Stellaria sp. } & 20 \quad \mathrm{H}\end{array}$

Bromus tectorum $2 \mathrm{H}$

$\begin{array}{lll}N A & 28 & \text { G }\end{array}$

Trifolium pratense $\quad 75 \quad \mathrm{H}$

Bromus inermus $\quad 25 \quad \mathrm{H}$ 


\begin{tabular}{|c|c|c|c|c|}
\hline SITE & COMMON NAME & SCIENTIFIC NAME & COVER & STRATUM \\
\hline 196 & Orchardgrass & Dactylis glomerata & 2 & $\mathrm{H}$ \\
\hline 196 & Sweet Clover & Melilotus sp. & 5 & $\mathrm{H}$ \\
\hline 196 & Aster & Aster sp. & 5 & $\mathrm{H}$ \\
\hline 196 & Lespedeza & Lespedeza sp. & 2 & $\mathrm{H}$ \\
\hline 198 & Fescue & Festuca arundinacea & 85 & $\mathrm{H}$ \\
\hline 198 & Broomsedge & Andropogon virginicus & 2 & $\mathrm{H}$ \\
\hline 198 & Aster & Aster sp. & 8 & $\mathrm{H}$ \\
\hline 198 & Red Clover & Trifolium pratense & 5 & $\mathrm{H}$ \\
\hline 199 & Smooth Brome & Bromus inermus & 85 & $\mathrm{H}$ \\
\hline 199 & Orchardgrass & Dactylis glomerata & 10 & $\mathrm{H}$ \\
\hline 199 & Blue Vervain & Verbena hastata & 5 & $\mathrm{H}$ \\
\hline 200 & Goldenrod & Solidago sp. & 40 & $\mathrm{H}$ \\
\hline 200 & Orchardgrass & Dactylis glomerata & 10 & $\mathrm{H}$ \\
\hline 200 & Rush & Juncus sp. & 15 & $\mathrm{H}$ \\
\hline 200 & Bare Ground & $N A$ & 25 & G \\
\hline 200 & Aster & Aster sp. & 5 & $\mathrm{H}$ \\
\hline 200 & Residue Covered Ground & $N A$ & 5 & G \\
\hline 201 & Smooth Brome & Bromus inermus & 20 & $\mathrm{H}$ \\
\hline 201 & Goldenrod & Solidago sp. & 5 & $\mathrm{H}$ \\
\hline 201 & Mixed Weeds & $N A$ & 25 & $\mathrm{H}$ \\
\hline 201 & Residue Covered Ground & $N A$ & 50 & G \\
\hline 202 & Orchardgrass & Dactylis glomerata & 20 & $\mathrm{H}$ \\
\hline 202 & Goldenrod & Solidago sp. & 5 & $\mathrm{H}$ \\
\hline 202 & Mixed Weeds & $N A$ & 25 & $\mathrm{H}$ \\
\hline & Residue Covered Ground and Bare & & & \\
\hline 202 & Ground & $N A$ & 50 & G \\
\hline 203 & Smooth Brome & Bromus inermus & 70 & $\mathrm{H}$ \\
\hline 203 & Orchardgrass & Dactylis glomerata & 10 & $\mathrm{H}$ \\
\hline 203 & Residue Covered Ground & $N A$ & 20 & G \\
\hline 205 & Fescue & Festuca arundinacea & 75 & $\mathrm{H}$ \\
\hline 205 & Timothy & Phleum pratense & 15 & $\mathrm{H}$ \\
\hline 206 & Perennial Rye & Lolium perenne & 100 & $\mathrm{H}$ \\
\hline 207 & Perennial Rye & Lolium perenne & 100 & $\mathrm{H}$ \\
\hline 208 & Perennial Rye & Lolium perenne & 97 & $\mathrm{H}$ \\
\hline 208 & Oats & Avena sativa & 3 & $\mathrm{H}$ \\
\hline 209 & Smooth Brome & Bromus inermus & 80 & $\mathrm{H}$ \\
\hline 209 & Orchardgrass & Dactylis glomerata & 15 & $\mathrm{H}$ \\
\hline 209 & Goldenrod & Solidago sp. & 15 & $\mathrm{H}$ \\
\hline 209 & Common Milkweed & Asclepias syriaca & 5 & $\mathrm{H}$ \\
\hline 209 & Autumn Olive & Elaeagnus umbellata & 10 & S \\
\hline 210 & Smooth Brome & Bromus inermus & 90 & $\mathrm{H}$ \\
\hline 210 & Goldenrod & Solidago sp. & 2 & $\mathrm{H}$ \\
\hline 210 & Curled Dock & Rumex crispus & 1 & $\mathrm{H}$ \\
\hline 210 & Residue Covered Ground & $N A$ & 7 & G \\
\hline 211 & Fescue & Festuca arundinacea & 80 & $\mathrm{H}$ \\
\hline
\end{tabular}


SITE

\section{1}

211

212

212

212

212

212

212

213

214

214

214

214

217

217

218

218

218

219

219

219

220

220

220

221
COMMON NAME

Smooth Brome

Red Clover

Smooth Brome

Red Clover

Goldenrod

Aster

Smartweed

Residue Covered Ground

Smooth Brome

Goldenrod

Fox Sedge

Oats

Fescue

Fescue

Smooth Brome

Fescue

Orchardgrass

Fox Sedge

Fescue

Smooth Brome

Bare Ground

White Clover

Fescue

Yarrow

Fescue

Fox Sedge

Goldenrod

Lespedeza

Fescue

Goldenrod

Orchardgrass

Lespedeza

Red Clover

Curled Dock

Fox Sedge

White Clover

Sweet Clover

Fescue

Bare Ground

Kentucky Bluegrass

Broomsedge

Yarrow

Goldenrod

Fescue
SCIENTIFIC NAME

Bromus inermus

Trifolium pratense

Bromus inermus

Trifolium pratense

Solidago $s p$.

Aster sp.

Polygonum pensylvanicum

NA

Bromus inermus

Solidago $s p$.

Carex vulpinoidea

Avena sativa

Festuca arundinacea

Festuca arundinacea

Bromus inermus

Festuca arundinacea

Dactylis glomerata

Carex vulpinoidea

Festuca arundinacea

Bromus inermus

$N A$

Trifolium repens

Festuca arundinacea

Achillea millefolium

Festuca arundinacea

Carex vulpinoidea

Solidago $s p$.

Lespedeza sp.

Festuca arundinacea

Solidago $s p$.

Dactylis glomerata

Lespedeza sp.

Trifolium pratense

Rumex crispus

Carex vulpinoidea

Trifolium repens

Melilotus sp.

Festuca arundinacea

NA

Poa pratensis

Andropogon virginicus

Achillea millefolium

Solidago sp.

Festuca arundinacea
COVER STRATUM

\begin{tabular}{|c|c|}
\hline COVER & STRATUM \\
\hline 15 & $\mathrm{H}$ \\
\hline 5 & $\mathrm{H}$ \\
\hline 70 & $\mathrm{H}$ \\
\hline 15 & $\mathrm{H}$ \\
\hline 5 & $\mathrm{H}$ \\
\hline 3 & $\mathrm{H}$ \\
\hline 2 & $\mathrm{H}$ \\
\hline 5 & $\mathrm{G}$ \\
\hline 100 & $\mathrm{H}$ \\
\hline 65 & $\mathrm{H}$ \\
\hline 10 & $\mathrm{H}$ \\
\hline 15 & $\mathrm{H}$ \\
\hline 10 & $\mathrm{H}$ \\
\hline 65 & $\mathrm{H}$ \\
\hline 35 & $\mathrm{H}$ \\
\hline 85 & $\mathrm{H}$ \\
\hline 10 & $\mathrm{H}$ \\
\hline 5 & $\mathrm{H}$ \\
\hline 50 & $\mathrm{H}$ \\
\hline 35 & $\mathrm{H}$ \\
\hline 15 & G \\
\hline 55 & $\mathrm{H}$ \\
\hline 30 & $\mathrm{H}$ \\
\hline 15 & $\mathrm{H}$ \\
\hline 85 & $\mathrm{H}$ \\
\hline 5 & $\mathrm{H}$ \\
\hline 5 & $\mathrm{H}$ \\
\hline 5 & $\mathrm{H}$ \\
\hline 35 & $\mathrm{H}$ \\
\hline 45 & $\mathrm{H}$ \\
\hline 5 & $\mathrm{H}$ \\
\hline 2 & $\mathrm{H}$ \\
\hline 3 & $\mathrm{H}$ \\
\hline 2 & $\mathrm{H}$ \\
\hline 3 & $\mathrm{H}$ \\
\hline 5 & $\mathrm{H}$ \\
\hline 30 & $\mathrm{H}$ \\
\hline 20 & $\mathrm{H}$ \\
\hline 50 & $G$ \\
\hline 40 & $\mathrm{H}$ \\
\hline 20 & $\mathrm{H}$ \\
\hline
\end{tabular}

\begin{tabular}{|c|c|}
\hline COVER & STRATUM \\
\hline 15 & $\mathrm{H}$ \\
\hline 5 & $\mathrm{H}$ \\
\hline 70 & $\mathrm{H}$ \\
\hline 15 & $\mathrm{H}$ \\
\hline 5 & $\mathrm{H}$ \\
\hline 3 & $\mathrm{H}$ \\
\hline 2 & $\mathrm{H}$ \\
\hline 5 & $\mathrm{G}$ \\
\hline 100 & $\mathrm{H}$ \\
\hline 65 & $\mathrm{H}$ \\
\hline 10 & $\mathrm{H}$ \\
\hline 15 & $\mathrm{H}$ \\
\hline 10 & $\mathrm{H}$ \\
\hline 65 & $\mathrm{H}$ \\
\hline 35 & $\mathrm{H}$ \\
\hline 85 & $\mathrm{H}$ \\
\hline 10 & $\mathrm{H}$ \\
\hline 5 & $\mathrm{H}$ \\
\hline 50 & $\mathrm{H}$ \\
\hline 35 & $\mathrm{H}$ \\
\hline 15 & G \\
\hline 55 & $\mathrm{H}$ \\
\hline 30 & $\mathrm{H}$ \\
\hline 15 & $\mathrm{H}$ \\
\hline 85 & $\mathrm{H}$ \\
\hline 5 & $\mathrm{H}$ \\
\hline 5 & $\mathrm{H}$ \\
\hline 5 & $\mathrm{H}$ \\
\hline 35 & $\mathrm{H}$ \\
\hline 45 & $\mathrm{H}$ \\
\hline 5 & $\mathrm{H}$ \\
\hline 2 & $\mathrm{H}$ \\
\hline 3 & $\mathrm{H}$ \\
\hline 2 & $\mathrm{H}$ \\
\hline 3 & $\mathrm{H}$ \\
\hline 5 & $\mathrm{H}$ \\
\hline 30 & $\mathrm{H}$ \\
\hline 20 & $\mathrm{H}$ \\
\hline 50 & $G$ \\
\hline 40 & $\mathrm{H}$ \\
\hline 20 & $\mathrm{H}$ \\
\hline
\end{tabular}

\begin{tabular}{|c|c|}
\hline COVER & STRATUM \\
\hline 15 & $\mathrm{H}$ \\
\hline 5 & $\mathrm{H}$ \\
\hline 70 & $\mathrm{H}$ \\
\hline 15 & $\mathrm{H}$ \\
\hline 5 & $\mathrm{H}$ \\
\hline 3 & $\mathrm{H}$ \\
\hline 2 & $\mathrm{H}$ \\
\hline 5 & $\mathrm{G}$ \\
\hline 100 & $\mathrm{H}$ \\
\hline 65 & $\mathrm{H}$ \\
\hline 10 & $\mathrm{H}$ \\
\hline 15 & $\mathrm{H}$ \\
\hline 10 & $\mathrm{H}$ \\
\hline 65 & $\mathrm{H}$ \\
\hline 35 & $\mathrm{H}$ \\
\hline 85 & $\mathrm{H}$ \\
\hline 10 & $\mathrm{H}$ \\
\hline 5 & $\mathrm{H}$ \\
\hline 50 & $\mathrm{H}$ \\
\hline 35 & $\mathrm{H}$ \\
\hline 15 & G \\
\hline 55 & $\mathrm{H}$ \\
\hline 30 & $\mathrm{H}$ \\
\hline 15 & $\mathrm{H}$ \\
\hline 85 & $\mathrm{H}$ \\
\hline 5 & $\mathrm{H}$ \\
\hline 5 & $\mathrm{H}$ \\
\hline 5 & $\mathrm{H}$ \\
\hline 35 & $\mathrm{H}$ \\
\hline 45 & $\mathrm{H}$ \\
\hline 5 & $\mathrm{H}$ \\
\hline 2 & $\mathrm{H}$ \\
\hline 3 & $\mathrm{H}$ \\
\hline 2 & $\mathrm{H}$ \\
\hline 3 & $\mathrm{H}$ \\
\hline 5 & $\mathrm{H}$ \\
\hline 30 & $\mathrm{H}$ \\
\hline 20 & $\mathrm{H}$ \\
\hline 50 & $G$ \\
\hline 40 & $\mathrm{H}$ \\
\hline 20 & $\mathrm{H}$ \\
\hline
\end{tabular}

\begin{tabular}{|c|c|}
\hline COVER & STRATUM \\
\hline 15 & $\mathrm{H}$ \\
\hline 5 & $\mathrm{H}$ \\
\hline 70 & $\mathrm{H}$ \\
\hline 15 & $\mathrm{H}$ \\
\hline 5 & $\mathrm{H}$ \\
\hline 3 & $\mathrm{H}$ \\
\hline 2 & $\mathrm{H}$ \\
\hline 5 & $\mathrm{G}$ \\
\hline 100 & $\mathrm{H}$ \\
\hline 65 & $\mathrm{H}$ \\
\hline 10 & $\mathrm{H}$ \\
\hline 15 & $\mathrm{H}$ \\
\hline 10 & $\mathrm{H}$ \\
\hline 65 & $\mathrm{H}$ \\
\hline 35 & $\mathrm{H}$ \\
\hline 85 & $\mathrm{H}$ \\
\hline 10 & $\mathrm{H}$ \\
\hline 5 & $\mathrm{H}$ \\
\hline 50 & $\mathrm{H}$ \\
\hline 35 & $\mathrm{H}$ \\
\hline 15 & G \\
\hline 55 & $\mathrm{H}$ \\
\hline 30 & $\mathrm{H}$ \\
\hline 15 & $\mathrm{H}$ \\
\hline 85 & $\mathrm{H}$ \\
\hline 5 & $\mathrm{H}$ \\
\hline 5 & $\mathrm{H}$ \\
\hline 5 & $\mathrm{H}$ \\
\hline 35 & $\mathrm{H}$ \\
\hline 45 & $\mathrm{H}$ \\
\hline 5 & $\mathrm{H}$ \\
\hline 2 & $\mathrm{H}$ \\
\hline 3 & $\mathrm{H}$ \\
\hline 2 & $\mathrm{H}$ \\
\hline 3 & $\mathrm{H}$ \\
\hline 5 & $\mathrm{H}$ \\
\hline 30 & $\mathrm{H}$ \\
\hline 20 & $\mathrm{H}$ \\
\hline 50 & $G$ \\
\hline 40 & $\mathrm{H}$ \\
\hline 20 & $\mathrm{H}$ \\
\hline
\end{tabular}

H

$\mathrm{H}$

(

$\mathrm{H}$

G

\begin{tabular}{|c|c|}
\hline COVER & STRATUM \\
\hline 15 & $\mathrm{H}$ \\
\hline 5 & $\mathrm{H}$ \\
\hline 70 & $\mathrm{H}$ \\
\hline 15 & $\mathrm{H}$ \\
\hline 5 & $\mathrm{H}$ \\
\hline 3 & $\mathrm{H}$ \\
\hline 2 & $\mathrm{H}$ \\
\hline 5 & $\mathrm{G}$ \\
\hline 100 & $\mathrm{H}$ \\
\hline 65 & $\mathrm{H}$ \\
\hline 10 & $\mathrm{H}$ \\
\hline 15 & $\mathrm{H}$ \\
\hline 10 & $\mathrm{H}$ \\
\hline 65 & $\mathrm{H}$ \\
\hline 35 & $\mathrm{H}$ \\
\hline 85 & $\mathrm{H}$ \\
\hline 10 & $\mathrm{H}$ \\
\hline 5 & $\mathrm{H}$ \\
\hline 50 & $\mathrm{H}$ \\
\hline 35 & $\mathrm{H}$ \\
\hline 15 & G \\
\hline 55 & $\mathrm{H}$ \\
\hline 30 & $\mathrm{H}$ \\
\hline 15 & $\mathrm{H}$ \\
\hline 85 & $\mathrm{H}$ \\
\hline 5 & $\mathrm{H}$ \\
\hline 5 & $\mathrm{H}$ \\
\hline 5 & $\mathrm{H}$ \\
\hline 35 & $\mathrm{H}$ \\
\hline 45 & $\mathrm{H}$ \\
\hline 5 & $\mathrm{H}$ \\
\hline 2 & $\mathrm{H}$ \\
\hline 3 & $\mathrm{H}$ \\
\hline 2 & $\mathrm{H}$ \\
\hline 3 & $\mathrm{H}$ \\
\hline 5 & $\mathrm{H}$ \\
\hline 30 & $\mathrm{H}$ \\
\hline 20 & $\mathrm{H}$ \\
\hline 50 & $G$ \\
\hline 40 & $\mathrm{H}$ \\
\hline 20 & $\mathrm{H}$ \\
\hline
\end{tabular}

\begin{tabular}{|c|c|}
\hline COVER & STRATUM \\
\hline 15 & $\mathrm{H}$ \\
\hline 5 & $\mathrm{H}$ \\
\hline 70 & $\mathrm{H}$ \\
\hline 15 & $\mathrm{H}$ \\
\hline 5 & $\mathrm{H}$ \\
\hline 3 & $\mathrm{H}$ \\
\hline 2 & $\mathrm{H}$ \\
\hline 5 & $\mathrm{G}$ \\
\hline 100 & $\mathrm{H}$ \\
\hline 65 & $\mathrm{H}$ \\
\hline 10 & $\mathrm{H}$ \\
\hline 15 & $\mathrm{H}$ \\
\hline 10 & $\mathrm{H}$ \\
\hline 65 & $\mathrm{H}$ \\
\hline 35 & $\mathrm{H}$ \\
\hline 85 & $\mathrm{H}$ \\
\hline 10 & $\mathrm{H}$ \\
\hline 5 & $\mathrm{H}$ \\
\hline 50 & $\mathrm{H}$ \\
\hline 35 & $\mathrm{H}$ \\
\hline 15 & G \\
\hline 55 & $\mathrm{H}$ \\
\hline 30 & $\mathrm{H}$ \\
\hline 15 & $\mathrm{H}$ \\
\hline 85 & $\mathrm{H}$ \\
\hline 5 & $\mathrm{H}$ \\
\hline 5 & $\mathrm{H}$ \\
\hline 5 & $\mathrm{H}$ \\
\hline 35 & $\mathrm{H}$ \\
\hline 45 & $\mathrm{H}$ \\
\hline 5 & $\mathrm{H}$ \\
\hline 2 & $\mathrm{H}$ \\
\hline 3 & $\mathrm{H}$ \\
\hline 2 & $\mathrm{H}$ \\
\hline 3 & $\mathrm{H}$ \\
\hline 5 & $\mathrm{H}$ \\
\hline 30 & $\mathrm{H}$ \\
\hline 20 & $\mathrm{H}$ \\
\hline 50 & $G$ \\
\hline 40 & $\mathrm{H}$ \\
\hline 20 & $\mathrm{H}$ \\
\hline
\end{tabular}

$10 \mathrm{H}$

\begin{tabular}{|c|c|}
\hline COVER & STRATUM \\
\hline 15 & $\mathrm{H}$ \\
\hline 5 & $\mathrm{H}$ \\
\hline 70 & $\mathrm{H}$ \\
\hline 15 & $\mathrm{H}$ \\
\hline 5 & $\mathrm{H}$ \\
\hline 3 & $\mathrm{H}$ \\
\hline 2 & $\mathrm{H}$ \\
\hline 5 & $\mathrm{G}$ \\
\hline 100 & $\mathrm{H}$ \\
\hline 65 & $\mathrm{H}$ \\
\hline 10 & $\mathrm{H}$ \\
\hline 15 & $\mathrm{H}$ \\
\hline 10 & $\mathrm{H}$ \\
\hline 65 & $\mathrm{H}$ \\
\hline 35 & $\mathrm{H}$ \\
\hline 85 & $\mathrm{H}$ \\
\hline 10 & $\mathrm{H}$ \\
\hline 5 & $\mathrm{H}$ \\
\hline 50 & $\mathrm{H}$ \\
\hline 35 & $\mathrm{H}$ \\
\hline 15 & G \\
\hline 55 & $\mathrm{H}$ \\
\hline 30 & $\mathrm{H}$ \\
\hline 15 & $\mathrm{H}$ \\
\hline 85 & $\mathrm{H}$ \\
\hline 5 & $\mathrm{H}$ \\
\hline 5 & $\mathrm{H}$ \\
\hline 5 & $\mathrm{H}$ \\
\hline 35 & $\mathrm{H}$ \\
\hline 45 & $\mathrm{H}$ \\
\hline 5 & $\mathrm{H}$ \\
\hline 2 & $\mathrm{H}$ \\
\hline 3 & $\mathrm{H}$ \\
\hline 2 & $\mathrm{H}$ \\
\hline 3 & $\mathrm{H}$ \\
\hline 5 & $\mathrm{H}$ \\
\hline 30 & $\mathrm{H}$ \\
\hline 20 & $\mathrm{H}$ \\
\hline 50 & $G$ \\
\hline 40 & $\mathrm{H}$ \\
\hline 20 & $\mathrm{H}$ \\
\hline
\end{tabular}

\begin{tabular}{|c|c|}
\hline COVER & STRATUM \\
\hline 15 & $\mathrm{H}$ \\
\hline 5 & $\mathrm{H}$ \\
\hline 70 & $\mathrm{H}$ \\
\hline 15 & $\mathrm{H}$ \\
\hline 5 & $\mathrm{H}$ \\
\hline 3 & $\mathrm{H}$ \\
\hline 2 & $\mathrm{H}$ \\
\hline 5 & $\mathrm{G}$ \\
\hline 100 & $\mathrm{H}$ \\
\hline 65 & $\mathrm{H}$ \\
\hline 10 & $\mathrm{H}$ \\
\hline 15 & $\mathrm{H}$ \\
\hline 10 & $\mathrm{H}$ \\
\hline 65 & $\mathrm{H}$ \\
\hline 35 & $\mathrm{H}$ \\
\hline 85 & $\mathrm{H}$ \\
\hline 10 & $\mathrm{H}$ \\
\hline 5 & $\mathrm{H}$ \\
\hline 50 & $\mathrm{H}$ \\
\hline 35 & $\mathrm{H}$ \\
\hline 15 & G \\
\hline 55 & $\mathrm{H}$ \\
\hline 30 & $\mathrm{H}$ \\
\hline 15 & $\mathrm{H}$ \\
\hline 85 & $\mathrm{H}$ \\
\hline 5 & $\mathrm{H}$ \\
\hline 5 & $\mathrm{H}$ \\
\hline 5 & $\mathrm{H}$ \\
\hline 35 & $\mathrm{H}$ \\
\hline 45 & $\mathrm{H}$ \\
\hline 5 & $\mathrm{H}$ \\
\hline 2 & $\mathrm{H}$ \\
\hline 3 & $\mathrm{H}$ \\
\hline 2 & $\mathrm{H}$ \\
\hline 3 & $\mathrm{H}$ \\
\hline 5 & $\mathrm{H}$ \\
\hline 30 & $\mathrm{H}$ \\
\hline 20 & $\mathrm{H}$ \\
\hline 50 & $G$ \\
\hline 40 & $\mathrm{H}$ \\
\hline 20 & $\mathrm{H}$ \\
\hline
\end{tabular}

\begin{tabular}{|c|c|}
\hline COVER & STRATUM \\
\hline 15 & $\mathrm{H}$ \\
\hline 5 & $\mathrm{H}$ \\
\hline 70 & $\mathrm{H}$ \\
\hline 15 & $\mathrm{H}$ \\
\hline 5 & $\mathrm{H}$ \\
\hline 3 & $\mathrm{H}$ \\
\hline 2 & $\mathrm{H}$ \\
\hline 5 & $\mathrm{G}$ \\
\hline 100 & $\mathrm{H}$ \\
\hline 65 & $\mathrm{H}$ \\
\hline 10 & $\mathrm{H}$ \\
\hline 15 & $\mathrm{H}$ \\
\hline 10 & $\mathrm{H}$ \\
\hline 65 & $\mathrm{H}$ \\
\hline 35 & $\mathrm{H}$ \\
\hline 85 & $\mathrm{H}$ \\
\hline 10 & $\mathrm{H}$ \\
\hline 5 & $\mathrm{H}$ \\
\hline 50 & $\mathrm{H}$ \\
\hline 35 & $\mathrm{H}$ \\
\hline 15 & G \\
\hline 55 & $\mathrm{H}$ \\
\hline 30 & $\mathrm{H}$ \\
\hline 15 & $\mathrm{H}$ \\
\hline 85 & $\mathrm{H}$ \\
\hline 5 & $\mathrm{H}$ \\
\hline 5 & $\mathrm{H}$ \\
\hline 5 & $\mathrm{H}$ \\
\hline 35 & $\mathrm{H}$ \\
\hline 45 & $\mathrm{H}$ \\
\hline 5 & $\mathrm{H}$ \\
\hline 2 & $\mathrm{H}$ \\
\hline 3 & $\mathrm{H}$ \\
\hline 2 & $\mathrm{H}$ \\
\hline 3 & $\mathrm{H}$ \\
\hline 5 & $\mathrm{H}$ \\
\hline 30 & $\mathrm{H}$ \\
\hline 20 & $\mathrm{H}$ \\
\hline 50 & $G$ \\
\hline 40 & $\mathrm{H}$ \\
\hline 20 & $\mathrm{H}$ \\
\hline
\end{tabular}

\begin{tabular}{|c|c|}
\hline COVER & STRATUM \\
\hline 15 & $\mathrm{H}$ \\
\hline 5 & $\mathrm{H}$ \\
\hline 70 & $\mathrm{H}$ \\
\hline 15 & $\mathrm{H}$ \\
\hline 5 & $\mathrm{H}$ \\
\hline 3 & $\mathrm{H}$ \\
\hline 2 & $\mathrm{H}$ \\
\hline 5 & $\mathrm{G}$ \\
\hline 100 & $\mathrm{H}$ \\
\hline 65 & $\mathrm{H}$ \\
\hline 10 & $\mathrm{H}$ \\
\hline 15 & $\mathrm{H}$ \\
\hline 10 & $\mathrm{H}$ \\
\hline 65 & $\mathrm{H}$ \\
\hline 35 & $\mathrm{H}$ \\
\hline 85 & $\mathrm{H}$ \\
\hline 10 & $\mathrm{H}$ \\
\hline 5 & $\mathrm{H}$ \\
\hline 50 & $\mathrm{H}$ \\
\hline 35 & $\mathrm{H}$ \\
\hline 15 & G \\
\hline 55 & $\mathrm{H}$ \\
\hline 30 & $\mathrm{H}$ \\
\hline 15 & $\mathrm{H}$ \\
\hline 85 & $\mathrm{H}$ \\
\hline 5 & $\mathrm{H}$ \\
\hline 5 & $\mathrm{H}$ \\
\hline 5 & $\mathrm{H}$ \\
\hline 35 & $\mathrm{H}$ \\
\hline 45 & $\mathrm{H}$ \\
\hline 5 & $\mathrm{H}$ \\
\hline 2 & $\mathrm{H}$ \\
\hline 3 & $\mathrm{H}$ \\
\hline 2 & $\mathrm{H}$ \\
\hline 3 & $\mathrm{H}$ \\
\hline 5 & $\mathrm{H}$ \\
\hline 30 & $\mathrm{H}$ \\
\hline 20 & $\mathrm{H}$ \\
\hline 50 & $G$ \\
\hline 40 & $\mathrm{H}$ \\
\hline 20 & $\mathrm{H}$ \\
\hline
\end{tabular}

\begin{tabular}{|c|c|}
\hline COVER & STRATUM \\
\hline 15 & $\mathrm{H}$ \\
\hline 5 & $\mathrm{H}$ \\
\hline 70 & $\mathrm{H}$ \\
\hline 15 & $\mathrm{H}$ \\
\hline 5 & $\mathrm{H}$ \\
\hline 3 & $\mathrm{H}$ \\
\hline 2 & $\mathrm{H}$ \\
\hline 5 & $\mathrm{G}$ \\
\hline 100 & $\mathrm{H}$ \\
\hline 65 & $\mathrm{H}$ \\
\hline 10 & $\mathrm{H}$ \\
\hline 15 & $\mathrm{H}$ \\
\hline 10 & $\mathrm{H}$ \\
\hline 65 & $\mathrm{H}$ \\
\hline 35 & $\mathrm{H}$ \\
\hline 85 & $\mathrm{H}$ \\
\hline 10 & $\mathrm{H}$ \\
\hline 5 & $\mathrm{H}$ \\
\hline 50 & $\mathrm{H}$ \\
\hline 35 & $\mathrm{H}$ \\
\hline 15 & G \\
\hline 55 & $\mathrm{H}$ \\
\hline 30 & $\mathrm{H}$ \\
\hline 15 & $\mathrm{H}$ \\
\hline 85 & $\mathrm{H}$ \\
\hline 5 & $\mathrm{H}$ \\
\hline 5 & $\mathrm{H}$ \\
\hline 5 & $\mathrm{H}$ \\
\hline 35 & $\mathrm{H}$ \\
\hline 45 & $\mathrm{H}$ \\
\hline 5 & $\mathrm{H}$ \\
\hline 2 & $\mathrm{H}$ \\
\hline 3 & $\mathrm{H}$ \\
\hline 2 & $\mathrm{H}$ \\
\hline 3 & $\mathrm{H}$ \\
\hline 5 & $\mathrm{H}$ \\
\hline 30 & $\mathrm{H}$ \\
\hline 20 & $\mathrm{H}$ \\
\hline 50 & $G$ \\
\hline 40 & $\mathrm{H}$ \\
\hline 20 & $\mathrm{H}$ \\
\hline
\end{tabular}

\begin{tabular}{|c|c|}
\hline COVER & STRATUM \\
\hline 15 & $\mathrm{H}$ \\
\hline 5 & $\mathrm{H}$ \\
\hline 70 & $\mathrm{H}$ \\
\hline 15 & $\mathrm{H}$ \\
\hline 5 & $\mathrm{H}$ \\
\hline 3 & $\mathrm{H}$ \\
\hline 2 & $\mathrm{H}$ \\
\hline 5 & $\mathrm{G}$ \\
\hline 100 & $\mathrm{H}$ \\
\hline 65 & $\mathrm{H}$ \\
\hline 10 & $\mathrm{H}$ \\
\hline 15 & $\mathrm{H}$ \\
\hline 10 & $\mathrm{H}$ \\
\hline 65 & $\mathrm{H}$ \\
\hline 35 & $\mathrm{H}$ \\
\hline 85 & $\mathrm{H}$ \\
\hline 10 & $\mathrm{H}$ \\
\hline 5 & $\mathrm{H}$ \\
\hline 50 & $\mathrm{H}$ \\
\hline 35 & $\mathrm{H}$ \\
\hline 15 & G \\
\hline 55 & $\mathrm{H}$ \\
\hline 30 & $\mathrm{H}$ \\
\hline 15 & $\mathrm{H}$ \\
\hline 85 & $\mathrm{H}$ \\
\hline 5 & $\mathrm{H}$ \\
\hline 5 & $\mathrm{H}$ \\
\hline 5 & $\mathrm{H}$ \\
\hline 35 & $\mathrm{H}$ \\
\hline 45 & $\mathrm{H}$ \\
\hline 5 & $\mathrm{H}$ \\
\hline 2 & $\mathrm{H}$ \\
\hline 3 & $\mathrm{H}$ \\
\hline 2 & $\mathrm{H}$ \\
\hline 3 & $\mathrm{H}$ \\
\hline 5 & $\mathrm{H}$ \\
\hline 30 & $\mathrm{H}$ \\
\hline 20 & $\mathrm{H}$ \\
\hline 50 & $G$ \\
\hline 40 & $\mathrm{H}$ \\
\hline 20 & $\mathrm{H}$ \\
\hline
\end{tabular}

\begin{tabular}{|c|c|}
\hline COVER & STRATUM \\
\hline 15 & $\mathrm{H}$ \\
\hline 5 & $\mathrm{H}$ \\
\hline 70 & $\mathrm{H}$ \\
\hline 15 & $\mathrm{H}$ \\
\hline 5 & $\mathrm{H}$ \\
\hline 3 & $\mathrm{H}$ \\
\hline 2 & $\mathrm{H}$ \\
\hline 5 & $\mathrm{G}$ \\
\hline 100 & $\mathrm{H}$ \\
\hline 65 & $\mathrm{H}$ \\
\hline 10 & $\mathrm{H}$ \\
\hline 15 & $\mathrm{H}$ \\
\hline 10 & $\mathrm{H}$ \\
\hline 65 & $\mathrm{H}$ \\
\hline 35 & $\mathrm{H}$ \\
\hline 85 & $\mathrm{H}$ \\
\hline 10 & $\mathrm{H}$ \\
\hline 5 & $\mathrm{H}$ \\
\hline 50 & $\mathrm{H}$ \\
\hline 35 & $\mathrm{H}$ \\
\hline 15 & G \\
\hline 55 & $\mathrm{H}$ \\
\hline 30 & $\mathrm{H}$ \\
\hline 15 & $\mathrm{H}$ \\
\hline 85 & $\mathrm{H}$ \\
\hline 5 & $\mathrm{H}$ \\
\hline 5 & $\mathrm{H}$ \\
\hline 5 & $\mathrm{H}$ \\
\hline 35 & $\mathrm{H}$ \\
\hline 45 & $\mathrm{H}$ \\
\hline 5 & $\mathrm{H}$ \\
\hline 2 & $\mathrm{H}$ \\
\hline 3 & $\mathrm{H}$ \\
\hline 2 & $\mathrm{H}$ \\
\hline 3 & $\mathrm{H}$ \\
\hline 5 & $\mathrm{H}$ \\
\hline 30 & $\mathrm{H}$ \\
\hline 20 & $\mathrm{H}$ \\
\hline 50 & $G$ \\
\hline 40 & $\mathrm{H}$ \\
\hline 20 & $\mathrm{H}$ \\
\hline
\end{tabular}

\begin{tabular}{|c|c|}
\hline COVER & STRATUM \\
\hline 15 & $\mathrm{H}$ \\
\hline 5 & $\mathrm{H}$ \\
\hline 70 & $\mathrm{H}$ \\
\hline 15 & $\mathrm{H}$ \\
\hline 5 & $\mathrm{H}$ \\
\hline 3 & $\mathrm{H}$ \\
\hline 2 & $\mathrm{H}$ \\
\hline 5 & $\mathrm{G}$ \\
\hline 100 & $\mathrm{H}$ \\
\hline 65 & $\mathrm{H}$ \\
\hline 10 & $\mathrm{H}$ \\
\hline 15 & $\mathrm{H}$ \\
\hline 10 & $\mathrm{H}$ \\
\hline 65 & $\mathrm{H}$ \\
\hline 35 & $\mathrm{H}$ \\
\hline 85 & $\mathrm{H}$ \\
\hline 10 & $\mathrm{H}$ \\
\hline 5 & $\mathrm{H}$ \\
\hline 50 & $\mathrm{H}$ \\
\hline 35 & $\mathrm{H}$ \\
\hline 15 & G \\
\hline 55 & $\mathrm{H}$ \\
\hline 30 & $\mathrm{H}$ \\
\hline 15 & $\mathrm{H}$ \\
\hline 85 & $\mathrm{H}$ \\
\hline 5 & $\mathrm{H}$ \\
\hline 5 & $\mathrm{H}$ \\
\hline 5 & $\mathrm{H}$ \\
\hline 35 & $\mathrm{H}$ \\
\hline 45 & $\mathrm{H}$ \\
\hline 5 & $\mathrm{H}$ \\
\hline 2 & $\mathrm{H}$ \\
\hline 3 & $\mathrm{H}$ \\
\hline 2 & $\mathrm{H}$ \\
\hline 3 & $\mathrm{H}$ \\
\hline 5 & $\mathrm{H}$ \\
\hline 30 & $\mathrm{H}$ \\
\hline 20 & $\mathrm{H}$ \\
\hline 50 & $G$ \\
\hline 40 & $\mathrm{H}$ \\
\hline 20 & $\mathrm{H}$ \\
\hline
\end{tabular}

\begin{tabular}{|c|c|}
\hline COVER & STRATUM \\
\hline 15 & $\mathrm{H}$ \\
\hline 5 & $\mathrm{H}$ \\
\hline 70 & $\mathrm{H}$ \\
\hline 15 & $\mathrm{H}$ \\
\hline 5 & $\mathrm{H}$ \\
\hline 3 & $\mathrm{H}$ \\
\hline 2 & $\mathrm{H}$ \\
\hline 5 & $\mathrm{G}$ \\
\hline 100 & $\mathrm{H}$ \\
\hline 65 & $\mathrm{H}$ \\
\hline 10 & $\mathrm{H}$ \\
\hline 15 & $\mathrm{H}$ \\
\hline 10 & $\mathrm{H}$ \\
\hline 65 & $\mathrm{H}$ \\
\hline 35 & $\mathrm{H}$ \\
\hline 85 & $\mathrm{H}$ \\
\hline 10 & $\mathrm{H}$ \\
\hline 5 & $\mathrm{H}$ \\
\hline 50 & $\mathrm{H}$ \\
\hline 35 & $\mathrm{H}$ \\
\hline 15 & G \\
\hline 55 & $\mathrm{H}$ \\
\hline 30 & $\mathrm{H}$ \\
\hline 15 & $\mathrm{H}$ \\
\hline 85 & $\mathrm{H}$ \\
\hline 5 & $\mathrm{H}$ \\
\hline 5 & $\mathrm{H}$ \\
\hline 5 & $\mathrm{H}$ \\
\hline 35 & $\mathrm{H}$ \\
\hline 45 & $\mathrm{H}$ \\
\hline 5 & $\mathrm{H}$ \\
\hline 2 & $\mathrm{H}$ \\
\hline 3 & $\mathrm{H}$ \\
\hline 2 & $\mathrm{H}$ \\
\hline 3 & $\mathrm{H}$ \\
\hline 5 & $\mathrm{H}$ \\
\hline 30 & $\mathrm{H}$ \\
\hline 20 & $\mathrm{H}$ \\
\hline 50 & $G$ \\
\hline 40 & $\mathrm{H}$ \\
\hline 20 & $\mathrm{H}$ \\
\hline
\end{tabular}

\begin{tabular}{|c|c|}
\hline COVER & STRATUM \\
\hline 15 & $\mathrm{H}$ \\
\hline 5 & $\mathrm{H}$ \\
\hline 70 & $\mathrm{H}$ \\
\hline 15 & $\mathrm{H}$ \\
\hline 5 & $\mathrm{H}$ \\
\hline 3 & $\mathrm{H}$ \\
\hline 2 & $\mathrm{H}$ \\
\hline 5 & $\mathrm{G}$ \\
\hline 100 & $\mathrm{H}$ \\
\hline 65 & $\mathrm{H}$ \\
\hline 10 & $\mathrm{H}$ \\
\hline 15 & $\mathrm{H}$ \\
\hline 10 & $\mathrm{H}$ \\
\hline 65 & $\mathrm{H}$ \\
\hline 35 & $\mathrm{H}$ \\
\hline 85 & $\mathrm{H}$ \\
\hline 10 & $\mathrm{H}$ \\
\hline 5 & $\mathrm{H}$ \\
\hline 50 & $\mathrm{H}$ \\
\hline 35 & $\mathrm{H}$ \\
\hline 15 & G \\
\hline 55 & $\mathrm{H}$ \\
\hline 30 & $\mathrm{H}$ \\
\hline 15 & $\mathrm{H}$ \\
\hline 85 & $\mathrm{H}$ \\
\hline 5 & $\mathrm{H}$ \\
\hline 5 & $\mathrm{H}$ \\
\hline 5 & $\mathrm{H}$ \\
\hline 35 & $\mathrm{H}$ \\
\hline 45 & $\mathrm{H}$ \\
\hline 5 & $\mathrm{H}$ \\
\hline 2 & $\mathrm{H}$ \\
\hline 3 & $\mathrm{H}$ \\
\hline 2 & $\mathrm{H}$ \\
\hline 3 & $\mathrm{H}$ \\
\hline 5 & $\mathrm{H}$ \\
\hline 30 & $\mathrm{H}$ \\
\hline 20 & $\mathrm{H}$ \\
\hline 50 & $G$ \\
\hline 40 & $\mathrm{H}$ \\
\hline 20 & $\mathrm{H}$ \\
\hline
\end{tabular}

\begin{tabular}{|c|c|}
\hline COVER & STRATUM \\
\hline 15 & $\mathrm{H}$ \\
\hline 5 & $\mathrm{H}$ \\
\hline 70 & $\mathrm{H}$ \\
\hline 15 & $\mathrm{H}$ \\
\hline 5 & $\mathrm{H}$ \\
\hline 3 & $\mathrm{H}$ \\
\hline 2 & $\mathrm{H}$ \\
\hline 5 & $\mathrm{G}$ \\
\hline 100 & $\mathrm{H}$ \\
\hline 65 & $\mathrm{H}$ \\
\hline 10 & $\mathrm{H}$ \\
\hline 15 & $\mathrm{H}$ \\
\hline 10 & $\mathrm{H}$ \\
\hline 65 & $\mathrm{H}$ \\
\hline 35 & $\mathrm{H}$ \\
\hline 85 & $\mathrm{H}$ \\
\hline 10 & $\mathrm{H}$ \\
\hline 5 & $\mathrm{H}$ \\
\hline 50 & $\mathrm{H}$ \\
\hline 35 & $\mathrm{H}$ \\
\hline 15 & G \\
\hline 55 & $\mathrm{H}$ \\
\hline 30 & $\mathrm{H}$ \\
\hline 15 & $\mathrm{H}$ \\
\hline 85 & $\mathrm{H}$ \\
\hline 5 & $\mathrm{H}$ \\
\hline 5 & $\mathrm{H}$ \\
\hline 5 & $\mathrm{H}$ \\
\hline 35 & $\mathrm{H}$ \\
\hline 45 & $\mathrm{H}$ \\
\hline 5 & $\mathrm{H}$ \\
\hline 2 & $\mathrm{H}$ \\
\hline 3 & $\mathrm{H}$ \\
\hline 2 & $\mathrm{H}$ \\
\hline 3 & $\mathrm{H}$ \\
\hline 5 & $\mathrm{H}$ \\
\hline 30 & $\mathrm{H}$ \\
\hline 20 & $\mathrm{H}$ \\
\hline 50 & $G$ \\
\hline 40 & $\mathrm{H}$ \\
\hline 20 & $\mathrm{H}$ \\
\hline
\end{tabular}

\begin{tabular}{|c|c|}
\hline COVER & STRATUM \\
\hline 15 & $\mathrm{H}$ \\
\hline 5 & $\mathrm{H}$ \\
\hline 70 & $\mathrm{H}$ \\
\hline 15 & $\mathrm{H}$ \\
\hline 5 & $\mathrm{H}$ \\
\hline 3 & $\mathrm{H}$ \\
\hline 2 & $\mathrm{H}$ \\
\hline 5 & $\mathrm{G}$ \\
\hline 100 & $\mathrm{H}$ \\
\hline 65 & $\mathrm{H}$ \\
\hline 10 & $\mathrm{H}$ \\
\hline 15 & $\mathrm{H}$ \\
\hline 10 & $\mathrm{H}$ \\
\hline 65 & $\mathrm{H}$ \\
\hline 35 & $\mathrm{H}$ \\
\hline 85 & $\mathrm{H}$ \\
\hline 10 & $\mathrm{H}$ \\
\hline 5 & $\mathrm{H}$ \\
\hline 50 & $\mathrm{H}$ \\
\hline 35 & $\mathrm{H}$ \\
\hline 15 & G \\
\hline 55 & $\mathrm{H}$ \\
\hline 30 & $\mathrm{H}$ \\
\hline 15 & $\mathrm{H}$ \\
\hline 85 & $\mathrm{H}$ \\
\hline 5 & $\mathrm{H}$ \\
\hline 5 & $\mathrm{H}$ \\
\hline 5 & $\mathrm{H}$ \\
\hline 35 & $\mathrm{H}$ \\
\hline 45 & $\mathrm{H}$ \\
\hline 5 & $\mathrm{H}$ \\
\hline 2 & $\mathrm{H}$ \\
\hline 3 & $\mathrm{H}$ \\
\hline 2 & $\mathrm{H}$ \\
\hline 3 & $\mathrm{H}$ \\
\hline 5 & $\mathrm{H}$ \\
\hline 30 & $\mathrm{H}$ \\
\hline 20 & $\mathrm{H}$ \\
\hline 50 & $G$ \\
\hline 40 & $\mathrm{H}$ \\
\hline 20 & $\mathrm{H}$ \\
\hline
\end{tabular}

\begin{tabular}{|c|c|}
\hline COVER & STRATUM \\
\hline 15 & $\mathrm{H}$ \\
\hline 5 & $\mathrm{H}$ \\
\hline 70 & $\mathrm{H}$ \\
\hline 15 & $\mathrm{H}$ \\
\hline 5 & $\mathrm{H}$ \\
\hline 3 & $\mathrm{H}$ \\
\hline 2 & $\mathrm{H}$ \\
\hline 5 & $\mathrm{G}$ \\
\hline 100 & $\mathrm{H}$ \\
\hline 65 & $\mathrm{H}$ \\
\hline 10 & $\mathrm{H}$ \\
\hline 15 & $\mathrm{H}$ \\
\hline 10 & $\mathrm{H}$ \\
\hline 65 & $\mathrm{H}$ \\
\hline 35 & $\mathrm{H}$ \\
\hline 85 & $\mathrm{H}$ \\
\hline 10 & $\mathrm{H}$ \\
\hline 5 & $\mathrm{H}$ \\
\hline 50 & $\mathrm{H}$ \\
\hline 35 & $\mathrm{H}$ \\
\hline 15 & G \\
\hline 55 & $\mathrm{H}$ \\
\hline 30 & $\mathrm{H}$ \\
\hline 15 & $\mathrm{H}$ \\
\hline 85 & $\mathrm{H}$ \\
\hline 5 & $\mathrm{H}$ \\
\hline 5 & $\mathrm{H}$ \\
\hline 5 & $\mathrm{H}$ \\
\hline 35 & $\mathrm{H}$ \\
\hline 45 & $\mathrm{H}$ \\
\hline 5 & $\mathrm{H}$ \\
\hline 2 & $\mathrm{H}$ \\
\hline 3 & $\mathrm{H}$ \\
\hline 2 & $\mathrm{H}$ \\
\hline 3 & $\mathrm{H}$ \\
\hline 5 & $\mathrm{H}$ \\
\hline 30 & $\mathrm{H}$ \\
\hline 20 & $\mathrm{H}$ \\
\hline 50 & $G$ \\
\hline 40 & $\mathrm{H}$ \\
\hline 20 & $\mathrm{H}$ \\
\hline
\end{tabular}

\begin{tabular}{|c|c|}
\hline COVER & STRATUM \\
\hline 15 & $\mathrm{H}$ \\
\hline 5 & $\mathrm{H}$ \\
\hline 70 & $\mathrm{H}$ \\
\hline 15 & $\mathrm{H}$ \\
\hline 5 & $\mathrm{H}$ \\
\hline 3 & $\mathrm{H}$ \\
\hline 2 & $\mathrm{H}$ \\
\hline 5 & $\mathrm{G}$ \\
\hline 100 & $\mathrm{H}$ \\
\hline 65 & $\mathrm{H}$ \\
\hline 10 & $\mathrm{H}$ \\
\hline 15 & $\mathrm{H}$ \\
\hline 10 & $\mathrm{H}$ \\
\hline 65 & $\mathrm{H}$ \\
\hline 35 & $\mathrm{H}$ \\
\hline 85 & $\mathrm{H}$ \\
\hline 10 & $\mathrm{H}$ \\
\hline 5 & $\mathrm{H}$ \\
\hline 50 & $\mathrm{H}$ \\
\hline 35 & $\mathrm{H}$ \\
\hline 15 & G \\
\hline 55 & $\mathrm{H}$ \\
\hline 30 & $\mathrm{H}$ \\
\hline 15 & $\mathrm{H}$ \\
\hline 85 & $\mathrm{H}$ \\
\hline 5 & $\mathrm{H}$ \\
\hline 5 & $\mathrm{H}$ \\
\hline 5 & $\mathrm{H}$ \\
\hline 35 & $\mathrm{H}$ \\
\hline 45 & $\mathrm{H}$ \\
\hline 5 & $\mathrm{H}$ \\
\hline 2 & $\mathrm{H}$ \\
\hline 3 & $\mathrm{H}$ \\
\hline 2 & $\mathrm{H}$ \\
\hline 3 & $\mathrm{H}$ \\
\hline 5 & $\mathrm{H}$ \\
\hline 30 & $\mathrm{H}$ \\
\hline 20 & $\mathrm{H}$ \\
\hline 50 & $G$ \\
\hline 40 & $\mathrm{H}$ \\
\hline 20 & $\mathrm{H}$ \\
\hline
\end{tabular}

\begin{tabular}{|c|c|}
\hline COVER & STRATUM \\
\hline 15 & $\mathrm{H}$ \\
\hline 5 & $\mathrm{H}$ \\
\hline 70 & $\mathrm{H}$ \\
\hline 15 & $\mathrm{H}$ \\
\hline 5 & $\mathrm{H}$ \\
\hline 3 & $\mathrm{H}$ \\
\hline 2 & $\mathrm{H}$ \\
\hline 5 & $\mathrm{G}$ \\
\hline 100 & $\mathrm{H}$ \\
\hline 65 & $\mathrm{H}$ \\
\hline 10 & $\mathrm{H}$ \\
\hline 15 & $\mathrm{H}$ \\
\hline 10 & $\mathrm{H}$ \\
\hline 65 & $\mathrm{H}$ \\
\hline 35 & $\mathrm{H}$ \\
\hline 85 & $\mathrm{H}$ \\
\hline 10 & $\mathrm{H}$ \\
\hline 5 & $\mathrm{H}$ \\
\hline 50 & $\mathrm{H}$ \\
\hline 35 & $\mathrm{H}$ \\
\hline 15 & G \\
\hline 55 & $\mathrm{H}$ \\
\hline 30 & $\mathrm{H}$ \\
\hline 15 & $\mathrm{H}$ \\
\hline 85 & $\mathrm{H}$ \\
\hline 5 & $\mathrm{H}$ \\
\hline 5 & $\mathrm{H}$ \\
\hline 5 & $\mathrm{H}$ \\
\hline 35 & $\mathrm{H}$ \\
\hline 45 & $\mathrm{H}$ \\
\hline 5 & $\mathrm{H}$ \\
\hline 2 & $\mathrm{H}$ \\
\hline 3 & $\mathrm{H}$ \\
\hline 2 & $\mathrm{H}$ \\
\hline 3 & $\mathrm{H}$ \\
\hline 5 & $\mathrm{H}$ \\
\hline 30 & $\mathrm{H}$ \\
\hline 20 & $\mathrm{H}$ \\
\hline 50 & $G$ \\
\hline 40 & $\mathrm{H}$ \\
\hline 20 & $\mathrm{H}$ \\
\hline
\end{tabular}

\begin{tabular}{|c|c|}
\hline COVER & STRATUM \\
\hline 15 & $\mathrm{H}$ \\
\hline 5 & $\mathrm{H}$ \\
\hline 70 & $\mathrm{H}$ \\
\hline 15 & $\mathrm{H}$ \\
\hline 5 & $\mathrm{H}$ \\
\hline 3 & $\mathrm{H}$ \\
\hline 2 & $\mathrm{H}$ \\
\hline 5 & $\mathrm{G}$ \\
\hline 100 & $\mathrm{H}$ \\
\hline 65 & $\mathrm{H}$ \\
\hline 10 & $\mathrm{H}$ \\
\hline 15 & $\mathrm{H}$ \\
\hline 10 & $\mathrm{H}$ \\
\hline 65 & $\mathrm{H}$ \\
\hline 35 & $\mathrm{H}$ \\
\hline 85 & $\mathrm{H}$ \\
\hline 10 & $\mathrm{H}$ \\
\hline 5 & $\mathrm{H}$ \\
\hline 50 & $\mathrm{H}$ \\
\hline 35 & $\mathrm{H}$ \\
\hline 15 & G \\
\hline 55 & $\mathrm{H}$ \\
\hline 30 & $\mathrm{H}$ \\
\hline 15 & $\mathrm{H}$ \\
\hline 85 & $\mathrm{H}$ \\
\hline 5 & $\mathrm{H}$ \\
\hline 5 & $\mathrm{H}$ \\
\hline 5 & $\mathrm{H}$ \\
\hline 35 & $\mathrm{H}$ \\
\hline 45 & $\mathrm{H}$ \\
\hline 5 & $\mathrm{H}$ \\
\hline 2 & $\mathrm{H}$ \\
\hline 3 & $\mathrm{H}$ \\
\hline 2 & $\mathrm{H}$ \\
\hline 3 & $\mathrm{H}$ \\
\hline 5 & $\mathrm{H}$ \\
\hline 30 & $\mathrm{H}$ \\
\hline 20 & $\mathrm{H}$ \\
\hline 50 & $G$ \\
\hline 40 & $\mathrm{H}$ \\
\hline 20 & $\mathrm{H}$ \\
\hline
\end{tabular}

\begin{tabular}{|c|c|}
\hline COVER & STRATUM \\
\hline 15 & $\mathrm{H}$ \\
\hline 5 & $\mathrm{H}$ \\
\hline 70 & $\mathrm{H}$ \\
\hline 15 & $\mathrm{H}$ \\
\hline 5 & $\mathrm{H}$ \\
\hline 3 & $\mathrm{H}$ \\
\hline 2 & $\mathrm{H}$ \\
\hline 5 & $\mathrm{G}$ \\
\hline 100 & $\mathrm{H}$ \\
\hline 65 & $\mathrm{H}$ \\
\hline 10 & $\mathrm{H}$ \\
\hline 15 & $\mathrm{H}$ \\
\hline 10 & $\mathrm{H}$ \\
\hline 65 & $\mathrm{H}$ \\
\hline 35 & $\mathrm{H}$ \\
\hline 85 & $\mathrm{H}$ \\
\hline 10 & $\mathrm{H}$ \\
\hline 5 & $\mathrm{H}$ \\
\hline 50 & $\mathrm{H}$ \\
\hline 35 & $\mathrm{H}$ \\
\hline 15 & G \\
\hline 55 & $\mathrm{H}$ \\
\hline 30 & $\mathrm{H}$ \\
\hline 15 & $\mathrm{H}$ \\
\hline 85 & $\mathrm{H}$ \\
\hline 5 & $\mathrm{H}$ \\
\hline 5 & $\mathrm{H}$ \\
\hline 5 & $\mathrm{H}$ \\
\hline 35 & $\mathrm{H}$ \\
\hline 45 & $\mathrm{H}$ \\
\hline 5 & $\mathrm{H}$ \\
\hline 2 & $\mathrm{H}$ \\
\hline 3 & $\mathrm{H}$ \\
\hline 2 & $\mathrm{H}$ \\
\hline 3 & $\mathrm{H}$ \\
\hline 5 & $\mathrm{H}$ \\
\hline 30 & $\mathrm{H}$ \\
\hline 20 & $\mathrm{H}$ \\
\hline 50 & $G$ \\
\hline 40 & $\mathrm{H}$ \\
\hline 20 & $\mathrm{H}$ \\
\hline
\end{tabular}

\begin{tabular}{|c|c|}
\hline COVER & STRATUM \\
\hline 15 & $\mathrm{H}$ \\
\hline 5 & $\mathrm{H}$ \\
\hline 70 & $\mathrm{H}$ \\
\hline 15 & $\mathrm{H}$ \\
\hline 5 & $\mathrm{H}$ \\
\hline 3 & $\mathrm{H}$ \\
\hline 2 & $\mathrm{H}$ \\
\hline 5 & $\mathrm{G}$ \\
\hline 100 & $\mathrm{H}$ \\
\hline 65 & $\mathrm{H}$ \\
\hline 10 & $\mathrm{H}$ \\
\hline 15 & $\mathrm{H}$ \\
\hline 10 & $\mathrm{H}$ \\
\hline 65 & $\mathrm{H}$ \\
\hline 35 & $\mathrm{H}$ \\
\hline 85 & $\mathrm{H}$ \\
\hline 10 & $\mathrm{H}$ \\
\hline 5 & $\mathrm{H}$ \\
\hline 50 & $\mathrm{H}$ \\
\hline 35 & $\mathrm{H}$ \\
\hline 15 & G \\
\hline 55 & $\mathrm{H}$ \\
\hline 30 & $\mathrm{H}$ \\
\hline 15 & $\mathrm{H}$ \\
\hline 85 & $\mathrm{H}$ \\
\hline 5 & $\mathrm{H}$ \\
\hline 5 & $\mathrm{H}$ \\
\hline 5 & $\mathrm{H}$ \\
\hline 35 & $\mathrm{H}$ \\
\hline 45 & $\mathrm{H}$ \\
\hline 5 & $\mathrm{H}$ \\
\hline 2 & $\mathrm{H}$ \\
\hline 3 & $\mathrm{H}$ \\
\hline 2 & $\mathrm{H}$ \\
\hline 3 & $\mathrm{H}$ \\
\hline 5 & $\mathrm{H}$ \\
\hline 30 & $\mathrm{H}$ \\
\hline 20 & $\mathrm{H}$ \\
\hline 50 & $G$ \\
\hline 40 & $\mathrm{H}$ \\
\hline 20 & $\mathrm{H}$ \\
\hline
\end{tabular}

\begin{tabular}{|c|c|}
\hline COVER & STRATUM \\
\hline 15 & $\mathrm{H}$ \\
\hline 5 & $\mathrm{H}$ \\
\hline 70 & $\mathrm{H}$ \\
\hline 15 & $\mathrm{H}$ \\
\hline 5 & $\mathrm{H}$ \\
\hline 3 & $\mathrm{H}$ \\
\hline 2 & $\mathrm{H}$ \\
\hline 5 & $\mathrm{G}$ \\
\hline 100 & $\mathrm{H}$ \\
\hline 65 & $\mathrm{H}$ \\
\hline 10 & $\mathrm{H}$ \\
\hline 15 & $\mathrm{H}$ \\
\hline 10 & $\mathrm{H}$ \\
\hline 65 & $\mathrm{H}$ \\
\hline 35 & $\mathrm{H}$ \\
\hline 85 & $\mathrm{H}$ \\
\hline 10 & $\mathrm{H}$ \\
\hline 5 & $\mathrm{H}$ \\
\hline 50 & $\mathrm{H}$ \\
\hline 35 & $\mathrm{H}$ \\
\hline 15 & G \\
\hline 55 & $\mathrm{H}$ \\
\hline 30 & $\mathrm{H}$ \\
\hline 15 & $\mathrm{H}$ \\
\hline 85 & $\mathrm{H}$ \\
\hline 5 & $\mathrm{H}$ \\
\hline 5 & $\mathrm{H}$ \\
\hline 5 & $\mathrm{H}$ \\
\hline 35 & $\mathrm{H}$ \\
\hline 45 & $\mathrm{H}$ \\
\hline 5 & $\mathrm{H}$ \\
\hline 2 & $\mathrm{H}$ \\
\hline 3 & $\mathrm{H}$ \\
\hline 2 & $\mathrm{H}$ \\
\hline 3 & $\mathrm{H}$ \\
\hline 5 & $\mathrm{H}$ \\
\hline 30 & $\mathrm{H}$ \\
\hline 20 & $\mathrm{H}$ \\
\hline 50 & $G$ \\
\hline 40 & $\mathrm{H}$ \\
\hline 20 & $\mathrm{H}$ \\
\hline
\end{tabular}

5

2

3

\begin{tabular}{|c|c|}
\hline COVER & STRATUM \\
\hline 15 & $\mathrm{H}$ \\
\hline 5 & $\mathrm{H}$ \\
\hline 70 & $\mathrm{H}$ \\
\hline 15 & $\mathrm{H}$ \\
\hline 5 & $\mathrm{H}$ \\
\hline 3 & $\mathrm{H}$ \\
\hline 2 & $\mathrm{H}$ \\
\hline 5 & $\mathrm{G}$ \\
\hline 100 & $\mathrm{H}$ \\
\hline 65 & $\mathrm{H}$ \\
\hline 10 & $\mathrm{H}$ \\
\hline 15 & $\mathrm{H}$ \\
\hline 10 & $\mathrm{H}$ \\
\hline 65 & $\mathrm{H}$ \\
\hline 35 & $\mathrm{H}$ \\
\hline 85 & $\mathrm{H}$ \\
\hline 10 & $\mathrm{H}$ \\
\hline 5 & $\mathrm{H}$ \\
\hline 50 & $\mathrm{H}$ \\
\hline 35 & $\mathrm{H}$ \\
\hline 15 & $\mathrm{G}$ \\
\hline 55 & $\mathrm{H}$ \\
\hline 30 & $\mathrm{H}$ \\
\hline 15 & $\mathrm{H}$ \\
\hline 85 & $\mathrm{H}$ \\
\hline 5 & $\mathrm{H}$ \\
\hline 5 & $\mathrm{H}$ \\
\hline 5 & $\mathrm{H}$ \\
\hline 35 & $\mathrm{H}$ \\
\hline 45 & $\mathrm{H}$ \\
\hline 5 & $\mathrm{H}$ \\
\hline 2 & $\mathrm{H}$ \\
\hline 3 & $\mathrm{H}$ \\
\hline 2 & $\mathrm{H}$ \\
\hline 3 & $\mathrm{H}$ \\
\hline 5 & $\mathrm{H}$ \\
\hline 30 & $\mathrm{H}$ \\
\hline 20 & $\mathrm{H}$ \\
\hline 50 & $G$ \\
\hline 40 & $\mathrm{H}$ \\
\hline 20 & $\mathrm{H}$ \\
\hline
\end{tabular}

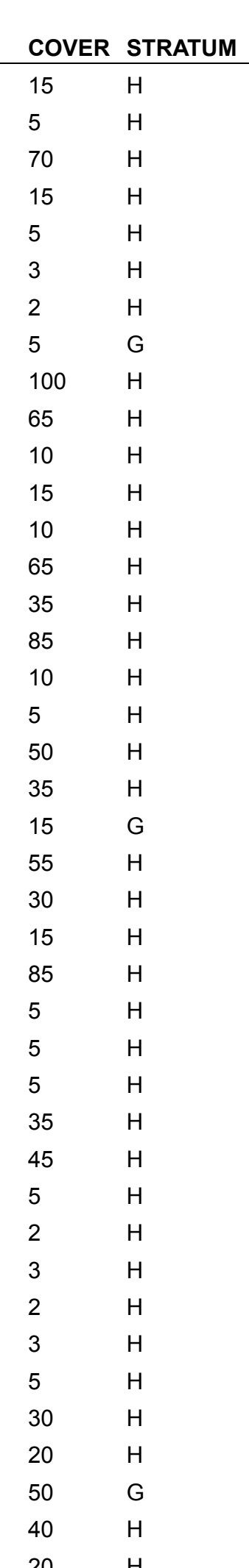

\begin{tabular}{|c|c|}
\hline COVER & STRATUM \\
\hline 15 & $\mathrm{H}$ \\
\hline 5 & $\mathrm{H}$ \\
\hline 70 & $\mathrm{H}$ \\
\hline 15 & $\mathrm{H}$ \\
\hline 5 & $\mathrm{H}$ \\
\hline 3 & $\mathrm{H}$ \\
\hline 2 & $\mathrm{H}$ \\
\hline 5 & G \\
\hline 100 & $\mathrm{H}$ \\
\hline 65 & $\mathrm{H}$ \\
\hline 10 & $\mathrm{H}$ \\
\hline 15 & $\mathrm{H}$ \\
\hline 10 & $\mathrm{H}$ \\
\hline 65 & $\mathrm{H}$ \\
\hline 35 & $\mathrm{H}$ \\
\hline 85 & $\mathrm{H}$ \\
\hline 10 & $\mathrm{H}$ \\
\hline 5 & $\mathrm{H}$ \\
\hline 50 & $\mathrm{H}$ \\
\hline 35 & $\mathrm{H}$ \\
\hline 15 & G \\
\hline 55 & $\mathrm{H}$ \\
\hline 30 & $\mathrm{H}$ \\
\hline 15 & $\mathrm{H}$ \\
\hline 85 & $\mathrm{H}$ \\
\hline 5 & $\mathrm{H}$ \\
\hline 5 & $\mathrm{H}$ \\
\hline 5 & $\mathrm{H}$ \\
\hline 35 & $\mathrm{H}$ \\
\hline 45 & $\mathrm{H}$ \\
\hline 5 & $\mathrm{H}$ \\
\hline 2 & $\mathrm{H}$ \\
\hline 3 & $\mathrm{H}$ \\
\hline 2 & $\mathrm{H}$ \\
\hline 3 & $\mathrm{H}$ \\
\hline 5 & $\mathrm{H}$ \\
\hline 30 & $\mathrm{H}$ \\
\hline 20 & $\mathrm{H}$ \\
\hline 50 & $\mathrm{G}$ \\
\hline 40 & $\mathrm{H}$ \\
\hline
\end{tabular}

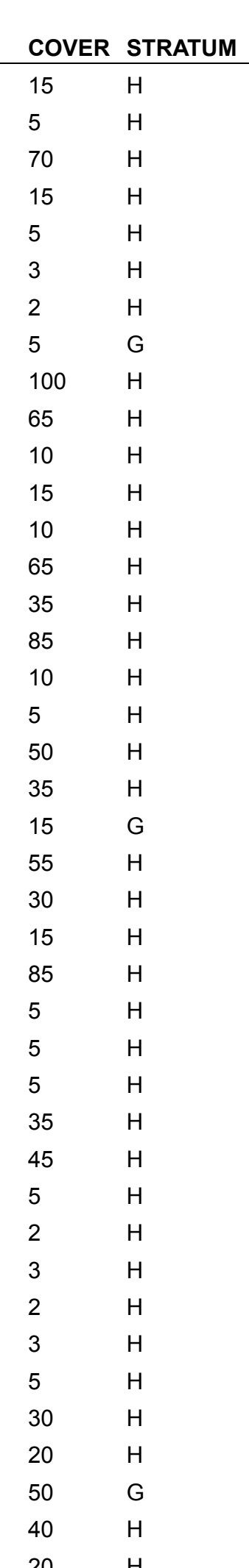

\begin{tabular}{|c|c|}
\hline COVER & STRATUM \\
\hline 15 & $\mathrm{H}$ \\
\hline 5 & $\mathrm{H}$ \\
\hline 70 & $\mathrm{H}$ \\
\hline 15 & $\mathrm{H}$ \\
\hline 5 & $\mathrm{H}$ \\
\hline 3 & $\mathrm{H}$ \\
\hline 2 & $\mathrm{H}$ \\
\hline 5 & G \\
\hline 100 & $\mathrm{H}$ \\
\hline 65 & $\mathrm{H}$ \\
\hline 10 & $\mathrm{H}$ \\
\hline 15 & $\mathrm{H}$ \\
\hline 10 & $\mathrm{H}$ \\
\hline 65 & $\mathrm{H}$ \\
\hline 35 & $\mathrm{H}$ \\
\hline 85 & $\mathrm{H}$ \\
\hline 10 & $\mathrm{H}$ \\
\hline 5 & $\mathrm{H}$ \\
\hline 50 & $\mathrm{H}$ \\
\hline 35 & $\mathrm{H}$ \\
\hline 15 & $\mathrm{G}$ \\
\hline 55 & $\mathrm{H}$ \\
\hline 30 & $\mathrm{H}$ \\
\hline 15 & $\mathrm{H}$ \\
\hline 85 & $\mathrm{H}$ \\
\hline 5 & $\mathrm{H}$ \\
\hline 5 & $\mathrm{H}$ \\
\hline 5 & $\mathrm{H}$ \\
\hline 35 & $\mathrm{H}$ \\
\hline 45 & $\mathrm{H}$ \\
\hline 5 & $\mathrm{H}$ \\
\hline 2 & $\mathrm{H}$ \\
\hline 3 & $\mathrm{H}$ \\
\hline 2 & $\mathrm{H}$ \\
\hline 3 & $\mathrm{H}$ \\
\hline 5 & $\mathrm{H}$ \\
\hline 30 & $\mathrm{H}$ \\
\hline 20 & $\mathrm{H}$ \\
\hline 50 & $\mathrm{G}$ \\
\hline 40 & $\mathrm{H}$ \\
\hline
\end{tabular}

\begin{tabular}{ll} 
COVER & STRATUM \\
\hline 15 & $\mathrm{H}$ \\
5 & $\mathrm{H}$ \\
70 & $\mathrm{H}$ \\
15 & $\mathrm{H}$ \\
5 & $\mathrm{H}$ \\
3 & $\mathrm{H}$ \\
2 & $\mathrm{H}$ \\
5 & $\mathrm{G}$ \\
100 & $\mathrm{H}$ \\
65 & $\mathrm{H}$ \\
10 & $\mathrm{H}$ \\
15 & $\mathrm{H}$ \\
10 & $\mathrm{H}$ \\
65 & $\mathrm{H}$ \\
35 & $\mathrm{H}$ \\
85 & $\mathrm{H}$ \\
10 & $\mathrm{H}$ \\
5 & $\mathrm{H}$ \\
50 & $\mathrm{H}$ \\
35 & $\mathrm{H}$ \\
15 & $\mathrm{G}$ \\
55 & $\mathrm{H}$ \\
30 & $\mathrm{H}$ \\
20 & $\mathrm{H}$ \\
15 & $\mathrm{H}$ \\
85 & $\mathrm{H}$ \\
5 & $\mathrm{H}$ \\
5 & $\mathrm{H}$ \\
5 & $\mathrm{H}$ \\
35 & $\mathrm{H}$ \\
45 & $\mathrm{H}$ \\
5 & $\mathrm{H}$ \\
2 & $\mathrm{H}$ \\
3 & $\mathrm{H}$ \\
2 & $\mathrm{H}$ \\
3 & $\mathrm{H}$ \\
5 & $\mathrm{H}$ \\
5 \\
5
\end{tabular}

\begin{tabular}{|c|c|}
\hline COVER & STRATUM \\
\hline 15 & $\mathrm{H}$ \\
\hline 5 & $\mathrm{H}$ \\
\hline 70 & $\mathrm{H}$ \\
\hline 15 & $\mathrm{H}$ \\
\hline 5 & $\mathrm{H}$ \\
\hline 3 & $\mathrm{H}$ \\
\hline 2 & $\mathrm{H}$ \\
\hline 5 & G \\
\hline 100 & $\mathrm{H}$ \\
\hline 65 & $\mathrm{H}$ \\
\hline 10 & $\mathrm{H}$ \\
\hline 15 & $\mathrm{H}$ \\
\hline 10 & $\mathrm{H}$ \\
\hline 65 & $\mathrm{H}$ \\
\hline 35 & $\mathrm{H}$ \\
\hline 85 & $\mathrm{H}$ \\
\hline 10 & $\mathrm{H}$ \\
\hline 5 & $\mathrm{H}$ \\
\hline 50 & $\mathrm{H}$ \\
\hline 35 & $\mathrm{H}$ \\
\hline 15 & $\mathrm{G}$ \\
\hline 55 & $\mathrm{H}$ \\
\hline 30 & $\mathrm{H}$ \\
\hline 15 & $\mathrm{H}$ \\
\hline 85 & $\mathrm{H}$ \\
\hline 5 & $\mathrm{H}$ \\
\hline 5 & $\mathrm{H}$ \\
\hline 5 & $\mathrm{H}$ \\
\hline 35 & $\mathrm{H}$ \\
\hline 45 & $\mathrm{H}$ \\
\hline 5 & $\mathrm{H}$ \\
\hline 2 & $\mathrm{H}$ \\
\hline 3 & $\mathrm{H}$ \\
\hline 2 & $\mathrm{H}$ \\
\hline 3 & $\mathrm{H}$ \\
\hline 5 & $\mathrm{H}$ \\
\hline 30 & $\mathrm{H}$ \\
\hline 20 & $\mathrm{H}$ \\
\hline 50 & $\mathrm{G}$ \\
\hline 40 & $\mathrm{H}$ \\
\hline
\end{tabular}

\begin{tabular}{ll} 
COVER & STRATUM \\
\hline 15 & $\mathrm{H}$ \\
5 & $\mathrm{H}$ \\
70 & $\mathrm{H}$ \\
15 & $\mathrm{H}$ \\
5 & $\mathrm{H}$ \\
3 & $\mathrm{H}$ \\
2 & $\mathrm{H}$ \\
5 & $\mathrm{G}$ \\
100 & $\mathrm{H}$ \\
65 & $\mathrm{H}$ \\
10 & $\mathrm{H}$ \\
15 & $\mathrm{H}$ \\
10 & $\mathrm{H}$ \\
65 & $\mathrm{H}$ \\
35 & $\mathrm{H}$ \\
85 & $\mathrm{H}$ \\
10 & $\mathrm{H}$ \\
5 & $\mathrm{H}$ \\
50 & $\mathrm{H}$ \\
35 & $\mathrm{H}$ \\
15 & $\mathrm{G}$ \\
55 & $\mathrm{H}$ \\
30 & $\mathrm{H}$ \\
20 & $\mathrm{H}$ \\
15 & $\mathrm{H}$ \\
85 & $\mathrm{H}$ \\
5 & $\mathrm{H}$ \\
5 & $\mathrm{H}$ \\
5 & $\mathrm{H}$ \\
35 & $\mathrm{H}$ \\
45 & $\mathrm{H}$ \\
5 & $\mathrm{H}$ \\
2 & $\mathrm{H}$ \\
3 & $\mathrm{H}$ \\
2 & $\mathrm{H}$ \\
3 & $\mathrm{H}$ \\
5 & $\mathrm{H}$ \\
5 \\
5
\end{tabular}

$20 \mathrm{H}$

$\begin{array}{ll}15 & \mathrm{H} \\ 2 & \mathrm{H}\end{array}$




\begin{tabular}{|c|c|c|c|c|}
\hline SITE & COMMON NAME & SCIENTIFIC NAME & COVER & STRATUM \\
\hline 224 & Residue Covered Ground & $N A$ & 18 & G \\
\hline 225 & Smooth Brome & Bromus inermus & 65 & $\mathrm{H}$ \\
\hline 225 & Common Milkweed & Asclepias syriaca & 1 & $\mathrm{H}$ \\
\hline 225 & Chickweed & Stellaria $s p$ & 2 & $\mathrm{H}$ \\
\hline 225 & Residue Covered Ground & $N A$ & 32 & G \\
\hline 226 & Green Ash & Fraxinus pennsylvanica & 30 & $\mathrm{~T} / \mathrm{S}$ \\
\hline 226 & Honey Locust & Gleditsia triacanthos & 20 & $\mathrm{~s}$ \\
\hline 226 & Fescue & Festuca arundinacea & 60 & $\mathrm{H}$ \\
\hline 226 & Smooth Brome & Bromus inermus & 40 & $\mathrm{H}$ \\
\hline 226 & Goldenrod & Solidago sp. & 2 & $\mathrm{H}$ \\
\hline 226 & Wild Carrot & Daucus carota & 20 & $\mathrm{H}$ \\
\hline 229 & Smooth Brome & Bromus inermus & 70 & $\mathrm{H}$ \\
\hline 229 & Residue Covered Ground & $N A$ & 30 & G \\
\hline 230 & Smooth Brome & Bromus inermus & 70 & $\mathrm{H}$ \\
\hline 230 & Residue Covered Ground & $N A$ & 30 & G \\
\hline 231 & Fescue & Festuca arundinacea & 10 & $\mathrm{H}$ \\
\hline 231 & Timothy & Phleum pratense & 55 & $\mathrm{H}$ \\
\hline 231 & Fox Sedge & Carex vulpinoidea & 15 & $\mathrm{H}$ \\
\hline 231 & Rush & Juncus sp. & 10 & $\mathrm{H}$ \\
\hline 231 & Residue Covered Ground & $N A$ & 10 & G \\
\hline 232 & Oats & Avena sativa & 85 & $\mathrm{H}$ \\
\hline 232 & Fescue & Festuca arundinacea & 15 & $\mathrm{H}$ \\
\hline 233 & Fescue & Festuca arundinacea & 80 & $\mathrm{H}$ \\
\hline 233 & Residue Covered Ground & $N A$ & 20 & G \\
\hline 235 & Lespedeza & Lespedeza sp. & 45 & $\mathrm{H}$ \\
\hline 235 & Fescue & Festuca arundinacea & 30 & $\mathrm{H}$ \\
\hline 235 & Red Clover & Trifolium pratense & 15 & $\mathrm{H}$ \\
\hline 235 & Yarrow & Achillea millefolium & 5 & $\mathrm{H}$ \\
\hline 235 & Orchardgrass & Dactylis glomerata & 5 & $\mathrm{H}$ \\
\hline 237 & Fescue & Festuca arundinacea & 94 & $\mathrm{H}$ \\
\hline 237 & Orchardgrass & Dactylis glomerata & 5 & $\mathrm{H}$ \\
\hline 237 & Korean Lespedeza & Lespedeza stipulacea & 1 & $\mathrm{H}$ \\
\hline 238 & Smooth Brome & Bromus inermus & 65 & $\mathrm{H}$ \\
\hline 238 & Wild Carrot & Daucus carota & 5 & $\mathrm{H}$ \\
\hline 238 & Goldenrod & Solidago $s p$ & 1 & $\mathrm{H}$ \\
\hline 238 & Cleavers & Galium aparine & 1 & $\mathrm{H}$ \\
\hline 238 & Wild Onion & Allium canadense & 1 & $\mathrm{H}$ \\
\hline 238 & Yarrow & Achillea millefolium & 1 & $\mathrm{H}$ \\
\hline 238 & Musk Thistle & Carduus nutans & 2 & $\mathrm{H}$ \\
\hline 238 & Bare Ground & $N A$ & 24 & G \\
\hline 239 & Smooth Brome & Bromus inermus & 98 & $\mathrm{H}$ \\
\hline 239 & Residue Covered Ground & $N A$ & 2 & G \\
\hline 240 & Smooth Brome & Bromus inermus & 85 & $\mathrm{H}$ \\
\hline 240 & Kentucky Bluegrass & Poa pratensis & 10 & $\mathrm{H}$ \\
\hline
\end{tabular}




\begin{tabular}{|c|c|c|c|c|}
\hline SITE & COMMON NAME & SCIENTIFIC NAME & COVER & STRATUM \\
\hline 240 & Residue Covered Ground & $N A$ & 5 & G \\
\hline 241 & Fescue & Festuca arundinacea & 90 & $\mathrm{H}$ \\
\hline 241 & Common Milkweed & Asclepias syriaca & 7 & $\mathrm{H}$ \\
\hline 241 & Multiflora Rose & Rosa multiflora & 5 & $S$ \\
\hline 241 & Late-Flowering Thoroughwort & Eupatorium serotinum & 3 & $\mathrm{H}$ \\
\hline 242 & Smooth Brome & Bromus inermus & 100 & $\mathrm{H}$ \\
\hline 243 & Smooth Brome & Bromus inermus & 80 & $\mathrm{H}$ \\
\hline 243 & Residue Covered Ground & $N A$ & 20 & G \\
\hline 246 & Fescue & Festuca arundinacea & 75 & $\mathrm{H}$ \\
\hline 246 & Autumn Olive & Elaeagnus umbellata & 1 & $S$ \\
\hline 246 & Residue Covered Ground & $N A$ & 24 & G \\
\hline 247 & Fescue & Festuca arundinacea & 80 & $\mathrm{H}$ \\
\hline 247 & Wood-Sorrel & Oxalis sp. & 1 & $\mathrm{H}$ \\
\hline 247 & Blackberry & Rubus allegheniensis & 2 & $\mathrm{H}$ \\
\hline 247 & Autumn Olive & Elaeagnus umbellata & 1 & $S$ \\
\hline 247 & Residue Covered Ground & $N A$ & 16 & G \\
\hline 248 & Fescue & Festuca arundinacea & 95 & $\mathrm{H}$ \\
\hline 248 & Orchardgrass & Dactylis glomerata & 3 & $\mathrm{H}$ \\
\hline 248 & Goldenrod & Solidago sp. & 2 & $\mathrm{H}$ \\
\hline 249 & Orchardgrass & Dactylis glomerata & 10 & $\mathrm{H}$ \\
\hline 249 & Kentucky Bluegrass & Poa pratensis & 40 & $\mathrm{H}$ \\
\hline 249 & Fescue & Festuca arundinacea & 5 & $\mathrm{H}$ \\
\hline 249 & Lespedeza & Lespedeza sp. & 25 & $\mathrm{H}$ \\
\hline 249 & Red Clover & Trifolium pratense & 10 & $\mathrm{H}$ \\
\hline 250 & Fescue & Festuca arundinacea & 40 & $\mathrm{H}$ \\
\hline 250 & Orchardgrass & Dactylis glomerata & 10 & $\mathrm{H}$ \\
\hline 250 & Lespedeza & Lespedeza sp. & 40 & $\mathrm{H}$ \\
\hline 250 & Kentucky Bluegrass & Poa pratensis & 8 & $\mathrm{H}$ \\
\hline 250 & Musk Thistle & Carduus nutans & 1 & $\mathrm{H}$ \\
\hline 251 & Orchardgrass & Dactylis glomerata & 40 & $\mathrm{H}$ \\
\hline 251 & Fescue & Festuca arundinacea & 20 & $\mathrm{H}$ \\
\hline 251 & Goldenrod & Solidago sp. & 5 & $\mathrm{H}$ \\
\hline 251 & Kentucky Bluegrass & Poa pratensis & 20 & $\mathrm{H}$ \\
\hline 251 & Lespedeza & Lespedeza sp. & 2 & $\mathrm{H}$ \\
\hline 251 & Red Clover & Trifolium pratense & 2 & $\mathrm{H}$ \\
\hline 251 & Bare Ground & $N A$ & 11 & G \\
\hline 252 & Smooth Brome & Bromus inermus & 60 & $\mathrm{H}$ \\
\hline 252 & Broomsedge & Andropogon virginicus & 1 & $\mathrm{H}$ \\
\hline 252 & Alfalfa & Medicago sativa & 1 & $\mathrm{H}$ \\
\hline 252 & Autumn Olive & Elaeagnus umbellata & 5 & $S$ \\
\hline 252 & Residue Covered Ground & $N A$ & 38 & G \\
\hline 253 & Smooth Brome & Bromus inermus & 85 & $\mathrm{H}$ \\
\hline 253 & Residue Covered Ground & $N A$ & 15 & G \\
\hline 254 & Fescue & Festuca arundinacea & 80 & $\mathrm{H}$ \\
\hline
\end{tabular}




\begin{tabular}{|c|c|c|c|c|}
\hline SITE & COMMON NAME & SCIENTIFIC NAME & COVER & STRATUM \\
\hline 254 & Kentucky Bluegrass & Poa pratensis & 20 & $\mathrm{H}$ \\
\hline 256 & Smooth Brome & Bromus inermus & 60 & $\mathrm{H}$ \\
\hline 256 & Orchardgrass & Dactylis glomerata & 40 & $\mathrm{H}$ \\
\hline 257 & Smooth Brome & Bromus inermus & 85 & $\mathrm{H}$ \\
\hline 257 & Honey Locust & Gleditsia triacanthos & 25 & S \\
\hline 257 & Residue Covered Ground & $N A$ & 15 & G \\
\hline 258 & Smooth Brome & Bromus inermus & 50 & $\mathrm{H}$ \\
\hline 258 & Kentucky Bluegrass & Poa pratensis & 50 & $\mathrm{H}$ \\
\hline 258 & Goldenrod & Solidago sp. & 20 & $\mathrm{H}$ \\
\hline 258 & Honey Locust & Gleditsia triacanthos & 50 & S \\
\hline 259 & Smooth Brome & Bromus inermus & 75 & $\mathrm{H}$ \\
\hline 259 & Ladino Clover & Trifolium repens latum & 10 & $\mathrm{H}$ \\
\hline 259 & Goldenrod & Solidago sp. & 5 & $\mathrm{H}$ \\
\hline 259 & Broomsedge & Andropogon virginicus & 1 & $\mathrm{H}$ \\
\hline 259 & Residue Covered Ground & $N A$ & 9 & G \\
\hline 260 & Smooth Brome & Bromus inermus & 90 & $\mathrm{H}$ \\
\hline 260 & Goldenrod & Solidago sp. & 5 & $\mathrm{H}$ \\
\hline 260 & Bare Ground & $N A$ & 5 & G \\
\hline 261 & Kentucky Bluegrass & Poa pratensis & 65 & $\mathrm{H}$ \\
\hline 261 & Blue Vervain & Verbena hastata & 20 & $\mathrm{H}$ \\
\hline 261 & Goldenrod & Solidago $s p$ & 2 & $\mathrm{H}$ \\
\hline 261 & Alfalfa & Medicago sativa & 1 & $\mathrm{H}$ \\
\hline 261 & Yarrow & Achillea millefolium & 1 & $\mathrm{H}$ \\
\hline 261 & Smooth Brome & Bromus inermus & 5 & $\mathrm{H}$ \\
\hline 261 & Autumn Olive & Elaeagnus umbellata & 1 & $S$ \\
\hline 261 & Bare Ground & $N A$ & 5 & G \\
\hline 262 & Oats & Avena sativa & 55 & $\mathrm{H}$ \\
\hline 262 & Fox Sedge & Carex vulpinoidea & 5 & $\mathrm{H}$ \\
\hline 262 & Goldenrod & Solidago $s p$ & 15 & $\mathrm{H}$ \\
\hline 262 & Foxtail & Setaria sp. & 2 & $\mathrm{H}$ \\
\hline 262 & Sedge & Carex sp. & 5 & $\mathrm{H}$ \\
\hline 262 & Alfalfa & Medicago sativa & 3 & $\mathrm{H}$ \\
\hline 262 & Kentucky Bluegrass & Poa pratensis & 3 & $\mathrm{H}$ \\
\hline 262 & Bare Ground & $N A$ & 12 & G \\
\hline 262 & Smooth Smooth Brome & Bromus inermus & 65 & $\mathrm{H}$ \\
\hline 263 & Aster & Aster sp. & 10 & $\mathrm{H}$ \\
\hline 263 & Goldenrod & Solidago sp. & 2 & $\mathrm{H}$ \\
\hline 263 & Foxglove Beardtongue & Penstemon digitalis & 1 & $\mathrm{H}$ \\
\hline 263 & Common Ragweed & Ambrosia artemisiifolia & 1 & $\mathrm{H}$ \\
\hline 263 & Sedge & Carex sp. & 1 & $\mathrm{H}$ \\
\hline & Residue Covered Ground and Bare & & & \\
\hline 263 & Ground & NA & 20 & G \\
\hline 264 & Smooth Brome & Bromus inermus & 60 & $\mathrm{H}$ \\
\hline 264 & Fox Sedge & Carex vulpinoidea & 10 & $\mathrm{H}$ \\
\hline 264 & Aster & Aster sp. & 5 & $\mathrm{H}$ \\
\hline
\end{tabular}




\begin{tabular}{|c|c|c|c|c|}
\hline SITE & COMMON NAME & SCIENTIFIC NAME & COVER & STRATUM \\
\hline 264 & Goldenrod & Solidago sp. & 10 & $\mathrm{H}$ \\
\hline 264 & Sedge & Carex sp. & 2 & $\mathrm{H}$ \\
\hline 264 & Bare Ground & $N A$ & 13 & G \\
\hline 265 & Smooth Brome & Bromus inermus & 75 & $\mathrm{H}$ \\
\hline 265 & Aster & Aster sp. & 3 & $\mathrm{H}$ \\
\hline 265 & Chickweed & Stellaria sp. & 1 & $\mathrm{H}$ \\
\hline 265 & St. John's Wort & Hypericum sp. & 2 & $\mathrm{H}$ \\
\hline 265 & Autumn Olive & Elaeagnus umbellata & 1 & $S$ \\
\hline 265 & Wild Geranium & Geranium maculatum & 1 & $\mathrm{H}$ \\
\hline 265 & Wood-Sorrel & Oxalis sp. & 1 & $\mathrm{H}$ \\
\hline 265 & Bare Ground & $N A$ & 16 & G \\
\hline 266 & Woolgrass & Scirpus cyperinus & 25 & $\mathrm{H}$ \\
\hline 266 & Fox Sedge & Carex vulpinoidea & 25 & $\mathrm{H}$ \\
\hline 266 & Goldenrod & Solidago sp. & 40 & $\mathrm{H}$ \\
\hline 266 & Smooth Brome & Bromus inermus & 10 & $\mathrm{H}$ \\
\hline 267 & American Elm & Ulmus americana & 30 & S \\
\hline 267 & Flowering Dogwood & Cornus florida & 40 & S \\
\hline 267 & Eastern Red Cedar & Juniperus virginiana & 5 & S \\
\hline 267 & Bush Honeysuckle & Lonicera sp. & 15 & S \\
\hline 267 & Japanese Honeysuckle & Lonicera japonica & 15 & $\mathrm{~V}$ \\
\hline 267 & Poison Ivy & Rhus radicans & 1 & $\mathrm{H}$ \\
\hline 267 & Agrimony & Agrimonia sp. & 20 & $\mathrm{H}$ \\
\hline 267 & Goldenrod & Solidago sp. & 3 & $\mathrm{H}$ \\
\hline 267 & Multiflora Rose & Rosa multiflora & 5 & $S$ \\
\hline 267 & Exposed leaf litter & $N A$ & 30 & G \\
\hline 269 & Wild Geranium & Geranium maculatum & 5 & $\mathrm{H}$ \\
\hline 269 & Wild Onion & Allium canadense & 5 & $\mathrm{H}$ \\
\hline 269 & Chickweed & Stellaria sp. & 5 & $\mathrm{H}$ \\
\hline 269 & Common Ragweed & Ambrosia artemisiifolia & 2 & $\mathrm{H}$ \\
\hline 269 & Shepard's-purse & Capsella bursa-pastoris & 53 & $\mathrm{H}$ \\
\hline 270 & Orchardgrass & Dactylis glomerata & 50 & $\mathrm{H}$ \\
\hline 270 & Fescue & Festuca arundinacea & 50 & $\mathrm{H}$ \\
\hline 270 & Honey Locust & Gleditsia triacanthos & 20 & $S$ \\
\hline 270 & Bush Honeysuckle & Lonicera sp. & 5 & S \\
\hline 270 & Hackberry & Celtis occidentalis & 2 & $S$ \\
\hline 270 & Sumac & Rhus sp. & 2 & S \\
\hline 271 & Orchardgrass & Dactylis glomerata & 65 & $\mathrm{H}$ \\
\hline 271 & Fescue & Festuca arundinacea & 35 & $\mathrm{H}$ \\
\hline
\end{tabular}




\section{Appendix C: Tree Field Data}

\begin{tabular}{|c|c|c|c|}
\hline SITE & COMMON NAME & SCIENTIFIC NAME & DBH (IN) \\
\hline 20 & Green Ash & Fraxinus pennsylvanica & 4 \\
\hline 20 & Green Ash & Fraxinus pennsylvanica & 6 \\
\hline 20 & Green Ash & Fraxinus pennsylvanica & 8 \\
\hline 20 & Honey Locust & Gleditsia triacanthos & 16 \\
\hline 20 & Honey Locust & Gleditsia triacanthos & 10 \\
\hline 20 & Osage Orange & Maclura pomifera & 6 \\
\hline 28 & Box Elder & Acer negundo & 14 \\
\hline 28 & Green Ash & Fraxinus pennsylvanica & 16 \\
\hline 28 & Hackberry & Celtis occidentalis & 12 \\
\hline 28 & Hackberry & Celtis occidentalis & 8 \\
\hline 28 & Red Elm & Ulmus rubra & 16 \\
\hline 28 & Red Elm & Ulmus rubra & 8 \\
\hline 28 & Red Elm & Ulmus rubra & 22 \\
\hline 28 & Red Elm & Ulmus rubra & 8 \\
\hline 28 & Red EIm & Ulmus rubra & 8 \\
\hline 28 & Red Elm & Ulmus rubra & 12 \\
\hline 28 & Silver Maple & Acer saccharinum & 20 \\
\hline 29 & Red Elm & Ulmus rubra & 6 \\
\hline 29 & Pin Oak & Quercus palustris & 22 \\
\hline 29 & Red Elm & Ulmus rubra & 14 \\
\hline 29 & Pin Oak & Quercus palustris & 18 \\
\hline 29 & Shingle Oak & Quercus imbricaria & 10 \\
\hline 29 & Red Elm & Ulmus rubra & 4 \\
\hline 29 & Green Ash & Fraxinus pennsylvanica & 8 \\
\hline 29 & Red Elm & Ulmus rubra & 8 \\
\hline 29 & Shingle Oak & Quercus imbricaria & 4 \\
\hline 29 & Hackberry & Celtis occidentalis & 22 \\
\hline 40 & Hawthorn & Crataegus sp. & 2 \\
\hline 40 & Osage Orange & Maclura pomifera & 16 \\
\hline 40 & Honey Locust & Gleditsia triacanthos & 18 \\
\hline 40 & Osage Orange & Maclura pomifera & 8 \\
\hline 40 & Hackberry & Celtis occidentalis & 10 \\
\hline 40 & Bitternut Hickory & Carya cordiformis & 12 \\
\hline 40 & American Elm & Ulmus americana & 18 \\
\hline 52 & Box Elder & Acer negundo & 4 \\
\hline 52 & Osage Orange & Maclura pomifera & 6 \\
\hline 52 & Ash & Fraxinus sp. & 8 \\
\hline 52 & Ash & Fraxinus sp. & 8 \\
\hline
\end{tabular}




\begin{tabular}{|c|c|c|c|}
\hline SITE & COMMON NAME & SCIENTIFIC NAME & DBH (IN) \\
\hline 52 & Sugarberry & Celtis laevigata & 8 \\
\hline 52 & Bitternut Hickory & Carya cordiformis & 8 \\
\hline 52 & Sugarberry & Celtis laevigata & 8 \\
\hline 52 & Ash & Fraxinus sp. & 8 \\
\hline 52 & Sugarberry & Celtis laevigata & 8 \\
\hline 62 & Silver Maple & Acer saccharinum & 26 \\
\hline 62 & Silver Maple & Acer saccharinum & 48 \\
\hline 62 & Shellbark Hickory & Carya laciniosa & 2 \\
\hline 62 & Shellbark Hickory & Carya laciniosa & 2 \\
\hline 62 & Osage Orange & Maclura pomifera & 6 \\
\hline 62 & Honey Locust & Gleditsia triacanthos & 18 \\
\hline 62 & Osage Orange & Maclura pomifera & 16 \\
\hline 62 & Osage Orange & Maclura pomifera & 24 \\
\hline 62 & Hackberry & Celtis occidentalis & 2 \\
\hline 93 & American Elm & Ulmus americana & 14 \\
\hline 93 & Green Ash & Fraxinus pennsylvanica & 8 \\
\hline 93 & Shellbark Hickory & Carya laciniosa & 8 \\
\hline 93 & Shellbark Hickory & Carya laciniosa & 10 \\
\hline 93 & Pin Oak & Quercus palustris & 18 \\
\hline 93 & Hackberry & Celtis occidentalis & 6 \\
\hline 93 & Green Ash & Fraxinus pennsylvanica & 10 \\
\hline 93 & Pin Oak & Quercus palustris & 24 \\
\hline 93 & Green Ash & Fraxinus pennsylvanica & 8 \\
\hline 93 & Pin Oak & Quercus palustris & 22 \\
\hline 93 & Pin Oak & Quercus palustris & 20 \\
\hline 104 & Green Ash & Fraxinus pennsylvanica & 8 \\
\hline 104 & Green Ash & Fraxinus pennsylvanica & 8 \\
\hline 104 & Box Elder & Acer negundo & 10 \\
\hline 104 & Green Ash & Fraxinus pennsylvanica & 24 \\
\hline 104 & Green Ash & Fraxinus pennsylvanica & 24 \\
\hline 104 & Silver Maple & Acer saccharinum & 6 \\
\hline 104 & Green Ash & Fraxinus pennsylvanica & 8 \\
\hline 104 & Silver Maple & Acer saccharinum & 6 \\
\hline 104 & Hackberry & Celtis occidentalis & 20 \\
\hline 104 & Green Ash & Fraxinus pennsylvanica & 14 \\
\hline 104 & Silver Maple & Acer saccharinum & 26 \\
\hline 104 & Silver Maple & Acer saccharinum & 22 \\
\hline 104 & Silver Maple & Acer saccharinum & 26 \\
\hline 116 & Silver Maple & Acer saccharinum & 5 \\
\hline 116 & River Birch & Betula nigra & 24 \\
\hline 116 & River Birch & Betula nigra & 18 \\
\hline 116 & River Birch & Betula nigra & 12 \\
\hline 116 & Green Ash & Fraxinus pennsylvanica & 12 \\
\hline 116 & Green Ash & Fraxinus pennsylvanica & 16 \\
\hline
\end{tabular}




\begin{tabular}{|c|c|c|c|}
\hline SITE & COMMON NAME & SCIENTIFIC NAME & DBH (IN) \\
\hline 116 & Elm & Ulmus sp. & 4 \\
\hline 116 & Silver Maple & Acer saccharinum & 22 \\
\hline 116 & Pin Oak & Quercus palustris & 20 \\
\hline 116 & Pin Oak & Quercus palustris & 20 \\
\hline 116 & Green Ash & Fraxinus pennsylvanica & 14 \\
\hline 116 & River Birch & Betula nigra & 16 \\
\hline 116 & River Birch & Betula nigra & 14 \\
\hline 128 & American Elm & Ulmus americana & 10 \\
\hline 128 & Osage Orange & Maclura pomifera & 8 \\
\hline 128 & Osage Orange & Maclura pomifera & 18 \\
\hline 128 & American Elm & Ulmus americana & 8 \\
\hline 128 & Osage Orange & Maclura pomifera & 10 \\
\hline 128 & American Elm & Ulmus americana & 6 \\
\hline 128 & Sugarberry & Celtis laevigata & 4 \\
\hline 128 & Honey Locust & Gleditsia triacanthos & 24 \\
\hline 128 & Osage Orange & Maclura pomifera & 20 \\
\hline 128 & Osage Orange & Maclura pomifera & 8 \\
\hline 138 & Sycamore & Platanus occidentalis & 18 \\
\hline 138 & Sycamore & Platanus occidentalis & 8 \\
\hline 138 & Sycamore & Platanus occidentalis & 16 \\
\hline 138 & Hackberry & Celtis occidentalis & 4 \\
\hline 138 & American Elm & Ulmus americana & 6 \\
\hline 138 & Osage Orange & Maclura pomifera & 14 \\
\hline 138 & Mulberry & Morus sp. & 12 \\
\hline 138 & Sycamore & Platanus occidentalis & 30 \\
\hline 138 & Osage Orange & Maclura pomifera & 10 \\
\hline 138 & Osage Orange & Maclura pomifera & 14 \\
\hline 138 & Box Elder & Acer negundo & 10 \\
\hline 166 & Silver Maple & Acer saccharinum & 14 \\
\hline 166 & Silver Maple & Acer saccharinum & 8 \\
\hline 166 & Silver Maple & Acer saccharinum & 16 \\
\hline 166 & Pin Oak & Quercus palustris & 16 \\
\hline 166 & Silver Maple & Acer saccharinum & 18 \\
\hline 166 & Silver Maple & Acer saccharinum & 16 \\
\hline 166 & Silver Maple & Acer saccharinum & 8 \\
\hline 166 & Green Ash & Fraxinus pennsylvanica & 8 \\
\hline 166 & Green Ash & Fraxinus pennsylvanica & 4 \\
\hline 166 & Hackberry & Celtis occidentalis & 2 \\
\hline 166 & Silver Maple & Acer saccharinum & 18 \\
\hline 167 & Silver Maple & Acer saccharinum & 8 \\
\hline 167 & Pin Oak & Quercus palustris & 18 \\
\hline 167 & Silver Maple & Acer saccharinum & 12 \\
\hline 167 & Green Ash & Fraxinus pennsylvanica & 2 \\
\hline 167 & Silver Maple & Acer saccharinum & 10 \\
\hline
\end{tabular}




\begin{tabular}{llll} 
SITE & COMMON NAME & SCIENTIFIC NAME & DBH (IN) \\
\hline 167 & Silver Maple & Acer saccharinum & 8 \\
167 & Silver Maple & Acer saccharinum & 10 \\
167 & Silver Maple & Acer saccharinum & 10 \\
167 & Silver Maple & Acer saccharinum & 6 \\
167 & Silver Maple & Acer saccharinum & 12 \\
167 & Silver Maple & Acer saccharinum & 8 \\
167 & Silver Maple & Acer saccharinum & 8 \\
167 & Pin Oak & Quercus palustris & 22 \\
168 & Black Walnut & Juglans nigra & 8 \\
168 & Siberian Elm & Ulmus pumila & 2 \\
168 & Siberian Elm & Ulmus pumila & 8 \\
168 & Siberian Elm & Ulmus pumila & 2 \\
168 & Siberian Elm & Ulmus pumila & 8 \\
168 & Siberian Elm & Ulmus pumila & 6 \\
168 & Siberian Elm & Ulmus pumila & 6
\end{tabular}




\title{
Appendix D: Field Notes
}

\author{
SITE NOTES \\ $9 \quad$ Yellow Billed Cuckoo sighted \\ 19 Very dense shrubs, herbaceous layer covered with Japanese Honeysuckle (Lonicera japonica) \\ Trees are in the surrounding area, approximate location, engineer could not get exact location \\ 19 because of dense shrubs \\ 30 agricultural field, treated with roundup and planted to Soybeans (Glycine max) \\ 31 agricultural field, planted to Soybeans (Glycine max) \\ 32 agricultural field, planted to Soybeans (Glycine max) \\ Hawthorn (Crataegus sp.) 2", Osage Orange 16" (Maclura pomifera), Honey Locust (Gleditsia \\ triacanthos) 18", Osage Orange 8", \\ Hackberry (Celtis occidentalis) 10", Bitternut Hickory (Carya cordiformis) 12", American Elm (UI- \\ $40 \quad$ mus americana) 18" \\ $41 \quad$ Sparse Vegetation at this site \\ Saplings: Sycamore (Platanus occidentalis), Greed Ash (Fraxinus sp.) and River Birch (Betula \\ nigra) \\ Shrub: Multiflora rose (Rosa multiflora) and Common Milkweed (Asclepias syriaca) \\ No Till Beans \\ No Till Beans \\ No Till Beans \\ Narrow-leaved Cattails (Typha angustifolia) and Slender Rush (Juncus tenuis) next to site \\ A lot of saplings in this area: Burr Oak (Quercus macrocarpa), Green Ash (Fraxinus pennsyl- \\ vanica) \\ Pin Oak (Quercus palustris), Box Elder (Acer negundo) \\ Ag. Field sprayed with Roundup \\ Honey Locust (Gleditsia triacanthos) 50\% cover, Persimmon (Diospyros virginiana) 20\% cover \\ both over plot, not in plot \\ 84 Shrubby, dense, grown-up vegetation next to woods \\ Site is adjacent to a small wetland dominated by Narrow-leaved Cattail (Typha angustifolia) $50 \%$ \\ and Common Reed (Phragmites australis), 50\% \\ $95 \quad$ Used GPS to locate, not staked or flagged \\ 96 GPS used to locate site (not staked) \\ 97 GPS used to locate site (not staked) \\ 104 In a depressional spot-bare soil.
}


SITE

106

113

116

\section{NOTES}

Site near a wetland.

Site is next to drain ravine into strip pit.

Plot adjacent to a wet slough.

Willow (Salix sp.) is next to strip pit adjacent to site.

Agricultural field, no-till (Corn last year) "Ag. Weeds" could not find stake, used GPS to find the site.

Agriculture field - No Lath, so used GPS to find the site. Corn stubble and agricultural weeds Agricultural field, no lath stake, located site with the GPS.

Site next to road.

Sparsely vegetated site.

No lath, so used GPS to locate site.

No lath, so used GPS to locate site, agricultural field (no-till) corn stubble.

Site next to road

Site on slope of a strip pit.

No exposed ground, all covered with leaf litter, sparse stand of vegetation.

Site is on a berm next to strip pit slope. Berm impounds water to form a small wetland.

Site next to limestone gravel road-gravelly site

Site in gravel road.

Gravelly site-next to limestone gravel road.

Common Milkweed (Asclepias syriaca)

Trumpet Creeper (Campsis radicans) on strip pit slope

1 Aster (Aster sp.), 1 Eastern Red Cedar (Juniperus virginiana), 1 Autumn Olive (Elaeagnus umbellata)

Sparse Vegetation at this site.

Site adjacent to road, cracked mud with vary sparse vegetation

Site located at the edge of the woods.

Autumn Olive (Elaeagnus umbellata) shrubs adjacent to site.

Autumn Olive (Elaeagnus umbellata) shrubs near site scattered about $25 \%$.

Sparse vegetation at this site.

Sparse vegetation at this site.

Field planted to Winter Wheat (Triticum aestivum).

Dense stand of autumn olive shrubs (Eleagnus umbellata) alder (Alnus sp.) and cypress trees (Taxodium distichum) next to strip pit.

Island of Fescue (Festuca arundinacea) surrounded by Perennial Rye (Lolium perenne) and Oats (Avena sativa).

Honey Locust (Gleditsia triacanthos), fairly dense, scattered along slope of ravine. Common Reed (Phragmites australis) near site, 
SITE

188

189

189

191

195

196

202

202

202

203

210

210

218 NOTES

1 Sycamore (Platanus occidentalis) and 1 Cottonwood (Populus deltoides).

Foxglove Beardtongue (Penstemon digitalis) and Mountain Mint (Pycanthemum tenuifolium) near plot,

also some Broomsedge (Andropogon virginicus).

Lots of grass residue. Early in season will be 100\% Fescue (Festuca arundinacea)

Sparse stand: planted in Winter Wheat (Triticum aestivum).

Dense vegetation cover, at least 2 quail calling.

Sparsely vegetated site.

*Mixed Weeds: Shepherd's Purse (Capsella bursa-pastoris), Yellow Wood-Sorrel (Oxalis sp.), Wild Onion (Allium sp.),

Aster (Aster sp.), Plantain sp., Rush (Juncus sp.), Common Ragweed (Ambrosia artemisiifolia). Somewhat sparse stand of vegetation.

Honey Locust (Gleditsia triacanthos) trees living edge of strip pit. Scattered Autumn Olive shrubs (Eleagnus umbellata) and Honey Locust trees

throughout the area surrounding the site.

One Musk Thistle (Carduus nutans) in plot.

By "Road Side" Gravelly-Limestone.

Autumn Olive (Elaeagnus umbellata) shrubs scattered thoughout area, Common Milkweed (Asclepias syriaca)

Goldedrod (Solidago sp.). Smooth Brome (Bromus inermus) not very dense

Steep slope of strip pit. Dense Vegetation over 100\% cover (species overlap).

Flushed 3 Mallards, Little green Heron and 2 Redwing Blackbirds from a wetland adjacent to the site.

Area around site mix of Fescue (Festuca arundinacea), Oats (Avena sativa) interspersed with volunteer Goldenrod (Solidago sp.),

Moth Mullein, Late-Flowering Thoroughwort (Eupatorium serotinum), Yarrow (Achillea millefolium), sparse Autumn Olive

(Elaeagnus umbellata) near top slope to strip pit $\sim 2$ yr old and a few Willow (Salix sp.) on the opposite shore with a lot of Meadowlarks and 2 Quail

Site next to road, near fence.

Smooth Brome (Bromus inermus) will fill in later in the season.

Multiflora Rose, (Rosa multiflora) is adjacent to plot but is hanging over this plot.

Couldn't find stake, used the GPS plugger to locate site coordinates.

Grass stunted at this site.

Honey Locust (Gleditsia triacanthos) trees next to plot on banks of strip pit (very steep banks). Autumn Olive (Eleagnus umbellata) 


\section{SITE NOTES}

and Honey Locust scattered around area, lots of Goldenrod (Solidago sp.), some Broomsedge

254 (Andropogon virginicus), Late-flowering

Late-Flowering Thoroughwort (Eupatorium serotinum), Blue Vervain (Verbena hastata) and Yar-

254 row (Achillea millefolium).

257 Site is adjacent to a ravine covered with Honey Locust (Gleditsia triacanthos)

258 Lots of Honey Locust (Gleditsia triacanthos) saplings, dense vegetation.

Common Ragweed is just coming up. Some exposed ground with leaf litter. Site is inundated 263 with about $1 / 2$ inch of water.

264 Half of site inundated $\sim 1-3 "$ ponded water

267 Lots of bush honeysuckle adjacent to plot. Understory sparse because of shade

270 Riparian Zone: 4 Canadian Geese in a low spot in open area between sites 269 and 270

These notes were taken during site visits during May and June 2002. 


\section{Appendix E: Soils Field Data}




\begin{tabular}{|c|c|c|c|c|c|c|c|c|c|c|c|}
\hline Site & Series & Slope & Aspect & Horizon & Depth & Moist Color & Texture & Structure & Consistency & $\mathbf{P h}$ & Boundary \\
\hline 7 & LENZBURG & 10 & NE & $A$ & $0-6$ & Mixed 10YR5/4\&5/1 & $\mathrm{CL}$ & $\mathrm{CP}$ & Vfirm & 6 & As \\
\hline 7 & LENZBURG & 10 & NE & $\mathrm{B} / \mathrm{C} 1$ & $6-18$ & Mixed 5/6,5/2\& 5/1 & CL 5\% & $\mathrm{CP}$ & Vfirm & $7.5 \mathrm{fc}$ & Cs \\
\hline 7 & LENZBURG & 10 & NE & $\mathrm{B} / \mathrm{C} 2$ & $18-60$ & Mixed 2.5Y6/2Y\& 10YR5/6,5/2 & CL 5\% & $\mathrm{CP}$ & Vfirm & $7.5 \mathrm{fc}$ & NA \\
\hline 8 & MORRISTOWN & 3 & W & Ap & $0-6$ & Mixed 5/6\&5/2 \& CFD5/8 & $\mathrm{CL}$ & $\mathrm{CP}$ & Vfirm & 5 & As \\
\hline 8 & MORRISTOWN & 3 & W & $\mathrm{B} / \mathrm{C} 1$ & $6-20$ & Mixed 5/2\&5/6 \& MFD5/8 & $\mathrm{CL}$ & $\mathrm{CP}$ & Vfirm & 5 & As \\
\hline 8 & MORRISTOWN & 3 & W & $\mathrm{B} / \mathrm{C} 2$ & $20-35$ & Mixed 5/2,5/1\&5/6 & $\mathrm{CL}$ & $\mathrm{CP}$ & Vfirm & 5 & As \\
\hline 9 & HOSMER & 2 & NA & Ap & $0-7$ & $5 / 3$ & SIL & $2 \mathrm{Fgr}$ & FR & 6 & As \\
\hline 9 & HOSMER & 2 & NA & $\mathrm{Bt} 1$ & $0-16$ & $5 / 4$ & SIL & 2Msbk & FR & 5.5 & Cs \\
\hline 9 & HOSMER & 2 & NA & $\mathrm{Bt} 2$ & $16-30$ & 5/4. CFD5/2\&5/8 & SICL & $2 \mathrm{Msbk}$ & FR & 5.5 & Cs \\
\hline 9 & HOSMER & 2 & NA & $\mathrm{Bt} 3$ & $30-40$ & 5/4. M5/2Y5/8 Cf5/3 & SICL & $2 \mathrm{Msbk}$ & FR & 5.5 & Cs \\
\hline 9 & HOSMER & 2 & NA & Bt4 & $40-52$ & 10YR5/3 \& CFF5/2,5/8 FEW CF5/2 & SICL & $2 \mathrm{Msbk}$ & FR & 5.5 & Cs \\
\hline 9 & HOSMER & 2 & NA & $\mathrm{BC}$ & $52-60$ & 10YR5/3 \& CFF5/2,5/8 & SICL & $2 \mathrm{Msbk}$ & FR & 6 & NA \\
\hline 10 & BIRDS & 0 & NA & Ap & $0-8$ & 10YR5/2 \& MMF4R, CFD5/8 & SIL & $2 F G R$ & FR & 5.5 & AS \\
\hline 10 & BIRDS & 0 & NA & $\mathrm{Bw}$ & $8-13$. & 10YR5/2 \& FMD10YR4/2,CFD5/8 & SIL & $2 \mathrm{FSBK}$ & FR & 5.5 & CS \\
\hline 10 & BIRDS & 0 & NA & $\mathrm{Cg} 1$ & $13-20$ & 10YR5/1 \& CFD5/8\&5/4 & SIL & $2 \mathrm{MSBK}$ & FR & 5 & CS \\
\hline 10 & BIRDS & 0 & NA & $\mathrm{Cg} 2$ & $20-39$ & 10YR6/2 \& CFD5/8\&5/2 & SIL & massive & FR & 5.5 & CS \\
\hline 10 & BIRDS & 0 & NA & $\mathrm{Cg} 2$ & $39-60$ & 10YR5/1 \& CMF2.5R6/2,CFD5/8 & SIL & massive & FR & 6 & CS \\
\hline 18 & LENZBURG & 2 & W & Ap & $0-8$ & Mixed 5/0 5/6,5/3 & $\mathrm{HvyCL}$ & $\mathrm{CP}$ & Vfirm & 5.5 & As \\
\hline
\end{tabular}




\begin{tabular}{|c|c|c|c|c|c|c|c|c|c|c|c|}
\hline Site & Series & Slope & Aspect & Horizon & Depth & Moist Color & Texture & Structure & Consistency & $\mathbf{P h}$ & Boundary \\
\hline 18 & LENZBURG & 2 & W & $\mathrm{B} / \mathrm{C} 1$ & $8-27$. & Mixed 5/6,5/1, 5/2 CFD 5/8 & $C L$ & $\mathrm{CP}$ & Vfirm & 6.5 & As \\
\hline 18 & LENZBURG & 2 & W & $\mathrm{B} / \mathrm{C} 2$ & $27-40$ & Mixed5/2,5/4,5/6 & $C L$ & $\mathrm{CP}$ & Vfirm & 6.5 & As \\
\hline 18 & LENZBURG & 2 & W & $\mathrm{B} / \mathrm{C} 3$ & $40-60$ & Mixed 5/4,5/1,5/6 & $\mathrm{CL}$ & $\mathrm{CP}$ & Vfirm & 7.5,FC & As \\
\hline 19 & HOSMER & 3 & $\mathrm{~S}$ & Ap & $0-8$ & 10YR4/3 & SIL & $2 \mathrm{Mgr}$ & FR & 6 & NA \\
\hline 19 & HOSMER & 3 & $S$ & Bt1 & $8-17$. & 10YR5/4 C CF4/4 & SICL & $2 \mathrm{Msbk}$ & $\mathrm{Fr}$ & 6 & NA \\
\hline 19 & HOSMER & 3 & $\mathrm{~S}$ & $B / E$ & $17-25$ & 10yr5/4 CFD5/8 MSC7/3 & SICL & $2 \mathrm{Msbk}$ & firm & 6 & NA \\
\hline 19 & HOSMER & 3 & $\mathrm{~S}$ & $B^{\prime} X$ & $25-36$ & 10YR5/4,MFD5/8,SC7/3,6/3 & SICL & $2 \mathrm{Msbk}$ & firm & 6 & NA \\
\hline 19 & HOSMER & 3 & $\mathrm{~S}$ & BTX & $36-50$ & NA & SICL & $2 \mathrm{Msbk}$ & firm & 6 & NA \\
\hline 19 & HOSMER & 3 & $\mathrm{~S}$ & C & $50-60$ & 10yr5/3,MFD5/8, 5/2 & SIL & $2 \mathrm{Msbk}$ & FR & 6 & NA \\
\hline 20 & WAKELAND & 0 & NA & A & $0-7$ & 10YR4/2 & SIL & $2 \mathrm{Fgr}$ & FR & $\mathrm{N}$ & CS \\
\hline 20 & WAKELAND & 0 & NA & Bw1 & $7-13$ & 10YR4/3,2F10 4/2 & SIL & 1Fsbk & FR & $\mathrm{N}$ & NA \\
\hline 20 & WAKELAND & 0 & NA & Bw2 & $13-30$ & 10YR5/2,10YR4/6,RCYM & SIL & $1 \mathrm{Msbk}$ & FR & $\mathrm{N}$ & NA \\
\hline 20 & WAKELAND & 0 & NA & C & $30-60$ & 10YR5/1, 4/6, 4/8 & SIL & $1 \mathrm{Msbk}$ & FR & $\mathrm{N}$ & NA \\
\hline 26 & LENZBURG & 10 & NE & A & $0-6$ & mixed $10 y r 5 / 4,5 / 1$ & $\mathrm{CL}$ & $\mathrm{cp}$ & Vfirm & 6 & As \\
\hline 26 & LENZBURG & 10 & NE & $\mathrm{B} / \mathrm{C} 1$ & $006-18$ & mixed $5 / 6,5 / 2,5 / 1$ & CL 5\% & $\mathrm{cp}$ & Vfirm & 7.5FC & Cs \\
\hline 26 & LENZBURG & 10 & NE & $\mathrm{B} / \mathrm{C} 2$ & $18-60$ & mixed 2.5 yr 6/2yr 10yr 5/6, 5/2 & CL 5\% & $\mathrm{cp}$ & Vfirm & 7.5FC & NA \\
\hline 28 & WAKELAND & 0 & NA & A & $0-5$ & $4 / 2$ & SIL & 2Fsbk & $\mathrm{Fr}$ & $\mathrm{N}$ & Cs \\
\hline 28 & WAKELAND & 0 & NA & Bw1 & $5-13$ & 10YR4/3 & SIL & 1Msbk & $\mathrm{Fr}$ & $\mathrm{N}$ & Cs \\
\hline
\end{tabular}




\begin{tabular}{|c|c|c|c|c|c|c|c|c|c|c|c|}
\hline Site & Series & Slope & Aspect & Horizon & Depth & Moist Color & Texture & Structure & Consistency & $\mathbf{P h}$ & Boundary \\
\hline 28 & WAKELAND & 0 & NA & BW2 & $13-35$ & $10 Y R 4 / 3,5 / 2 f 4 / 6$ & SIL & $1 \mathrm{Msbk}$ & $\mathrm{Fr}$ & $\mathrm{N}$ & Cs \\
\hline 28 & WAKELAND & 0 & NA & $\mathrm{C} 1$ & $35-50$ & 10YR4/3, M5/2\&4/6 & SIL & M & $\mathrm{Fr}$ & $\mathrm{N}$ & Cs \\
\hline 28 & WAKELAND & 0 & NA & $\mathrm{C} 2$ & $50-60$ & 10YR5/2,C4/3\&6/8 & SIL & M & $\mathrm{Fr}$ & $\mathrm{N}$ & Cs \\
\hline 29 & WILBUR & 0 & NA & Ap & $0-8$ & 10YR4/2 & SIL & $2 \mathrm{Fgr}$ & $\mathrm{F}$ & $\mathrm{N}$ & Cs \\
\hline 29 & WILBUR & 0 & NA & Bw1 & 8-17. & 10YR4/3 & SIL & 1Fsbk & $\mathrm{F}$ & $\mathrm{N}$ & Cs \\
\hline 29 & WILBUR & 0 & NA & Bw2 & $17-30$ & $10 \mathrm{yr} 4 / 3, \mathrm{w} 4 / 2 \mathrm{y} 4 / 6 \mathrm{RC}$ & SIL & $1 \mathrm{Msbk}$ & $\mathrm{F}$ & $\mathrm{N}$ & Cs \\
\hline 29 & WILBUR & 0 & NA & C & $30-42$ & $10 \mathrm{yr} 5 / 1, \mathrm{M} 4 / 2,6 / 8 \mathrm{RC}$ & SIL & Massive & $\mathrm{F}$ & $\mathrm{N}$ & Cs \\
\hline 29 & WILBUR & 0 & NA & C & $42-60$ & $10 \mathrm{yr} 6 / 1,6 / 4,5 / 8,4 / 3$ & SIL & Massive & $\mathrm{F}$ & $\mathrm{N}$ & NA \\
\hline 30 & STOY & 0 & NA & Ap & $0-7$ & 10YR4/3 & SIL & $2 \mathrm{Fgr}$ & $\mathrm{Fr}$ & 6 & As \\
\hline 30 & STOY & 0 & NA & $E$ & $7-13$ & $10 y r 5 / 3$ & sil & 1 fpty & $\mathrm{fr}$ & 6 & cs \\
\hline 30 & STOY & 0 & NA & Bt1 & $13-22$ & 10YR5/3,ffd 5/6,5/2 & sicl & $2 \mathrm{msbk}$ & FR & 6 & CS \\
\hline 30 & STOY & 0 & NA & bt2 & $22-30$ & $10 \mathrm{yr} 5 / 2$, cfd $5 / 8,5 / 4$ & sicl & $2 m s b k$ & FIRM & 6 & CS \\
\hline 30 & STOY & 0 & NA & $\operatorname{Btg} 1$ & $30-45$ & $10 y r 5 / 1$, cfd $5 / 8,5 / 4$ & sicl & $2 \mathrm{msbk}$ & $f$ & 6 & CS \\
\hline 30 & STOY & 0 & NA & Btg2 & $45-60$ & $10 y r 5 / 1$, cfd $5 / 8,5 / 4$ & sil & $2 \mathrm{fmsbk}$ & $\mathrm{fr}$ & 6 & CS \\
\hline 31 & SCHULINE & 2 & $\mathrm{n} / \mathrm{nw}$ & Ap & $0-4$ & $10 Y R 4 / 2 \& 4 / 3$ & SIL & Wfsbk & $\mathrm{Fr}$ & 6 & As \\
\hline 31 & SCHULINE & 2 & $\mathrm{n} / \mathrm{nw}$ & $\mathrm{B} / \mathrm{C} 1$ & $4-11$ & 10YR5/1, 5/4, \&4/3 & SIL\&SICL & Mass.CP & Firm & 6.5 & As \\
\hline 31 & SCHULINE & 2 & $\mathrm{n} / \mathrm{nw}$ & $\mathrm{B} / \mathrm{C} 2$ & $11-18$ & 10YR4/2 \& 5/1 & SIL\&SICL & Mass.CP & Firm & 7 & As \\
\hline 31 & SCHULINE & 2 & $\mathrm{n} / \mathrm{nw}$ & $\mathrm{B} / \mathrm{C} 3$ & $18-38$ & 10YR5/6, 5/2,\& 5/1 & CL\&SICL & Mass.CP & Firm & 7 & As \\
\hline
\end{tabular}




\begin{tabular}{|c|c|c|c|c|c|c|c|c|c|c|c|}
\hline Site & Series & Slope & Aspect & Horizon & Depth & Moist Color & Texture & Structure & Consistency & $\mathbf{P h}$ & Boundary \\
\hline 31 & SCHULINE & 2 & $\mathrm{n} / \mathrm{nw}$ & $\mathrm{B} / \mathrm{C} 4$ & $38-60$ & 10YR5/6, 4/1, \& 5/1 & $\mathrm{CL}$ & Mass.CP & Firm & 7 & NA \\
\hline 32 & MORRISTOWN & 6 & NE & Ap & $0-5$ & 10yr5/1, 5/6,CFD5/8 & C Loam & $\mathrm{CP}, \mathrm{M}$ & Vfirm & 5 & As \\
\hline 32 & MORRISTOWN & 6 & NE & $\mathrm{B} / \mathrm{C} 1$ & $5-25$ & $5 Y 5 / 1,5 / 6, \& 5 / 3$ & C\&CL & $\mathrm{CP}, \mathrm{M}$ & Vfirm & 5 & NA \\
\hline 32 & MORRISTOWN & 6 & NE & $\mathrm{B} / \mathrm{C} 2$ & $25-60$ & 5Y4/1, 10YR5/1 & Clayshale & $\mathrm{CP}$ & Vfirm & $7.5, \mathrm{FC}$ & As \\
\hline 35 & MORRISTOWN & 2 & SW & Ap & $0-5$ & Mixed 10YR3/1\&4/2 & SIL & 1FGR & $\mathrm{Fr}$ & 6 & As \\
\hline 35 & MORRISTOWN & 2 & SW & $\mathrm{B} / \mathrm{C} 1$ & $5-12$ & Mixed 10YR4/2\&5/3 & SIL & $1 \mathrm{Msbk}$ & $\mathrm{Fr}$ & $7.5 \mathrm{FC}$ & As \\
\hline 35 & MORRISTOWN & 2 & SW & $\mathrm{B} / \mathrm{C} 2$ & $12-32$ & Mixed 10yr6/1,5/2,5/6 & CL X, & $\mathrm{Cp}$ & Vfirm & $7.5, \mathrm{FC}$ & As \\
\hline 35 & MORRISTOWN & 2 & SW & $\mathrm{B} / \mathrm{C} 3$ & $32-60$ & Mixed 10YR4/1, & Shale & $\mathrm{CP}$ & Vfirm & $7.5, \mathrm{FC}$ & NA \\
\hline 36 & MORRISTOWN & 13 & $\mathrm{~N}$ & Ap & $0-6$ & Mixed 10YR4/2\&5/6 & SIL\&SICL & 1Fsbk & $\mathrm{Fr}$ & 6 & As \\
\hline 36 & MORRISTOWN & 13 & $\mathrm{~N}$ & $\mathrm{~B} / \mathrm{C}$ & $006-30$ & Mixed 10YR5/1\&5/6 & CL\&5\% & $\mathrm{CP}$ & Vfirm & $7.5, \mathrm{FC}$ & NA \\
\hline 39 & HURST & 0 & NA & Ap & $0-8$ & 10YR5/2,Ffd 5/8 & SIL & $2 F G R$ & $\mathrm{Fr}$ & 5.5 & Cs \\
\hline 39 & HURST & 0 & NA & $\mathrm{Bt} 1$ & $8-12$ & 10YR5/4, Cfd5/8\&5/2 & $\mathrm{HSICL}$ & $2 \mathrm{Msbk}$ & Firm & 5.5 & Cs \\
\hline 39 & HURST & 0 & NA & Btg1 & $12-28$ & 10yr5/2,Cmd5/8,CF5/1 5/4 & $\mathrm{HSICL}$ & $2 \mathrm{Msbk}$ & Firm & 6 & Cs \\
\hline 39 & HURST & 0 & NA & Btg2 & $28-40$ & $10 y r 5 / 1, C m d 5 / 8,4 / 6$ & $\mathrm{HSICL}$ & $2 \mathrm{Msbk}$ & Firm & 6 & Cs \\
\hline 39 & HURST & 0 & NA & Btg3 & $40-60$ & 2.5y6/2 CMD5/8,4/6,CF5/1 & SICL & $2 \mathrm{Msbk}$ & Firm & $7.5 \mathrm{FC}$ & Cs \\
\hline 40 & WAKELAND & 0 & NA & $A$ & $0-8$ & 10YR4/2 & SIL & $2 \mathrm{Fgr}$ & $\mathrm{Fr}$ & $\mathrm{N}$ & Cs \\
\hline 40 & WAKELAND & 0 & NA & Bw1 & $8-16$. & 10YR4/3 5/2\&5/8 & SIL & $1 \mathrm{Msbk}$ & $\mathrm{Fr}$ & $\mathrm{N}$ & Cs \\
\hline 40 & WAKELAND & 0 & NA & Bw2g & $16-30$ & 10YR5/2 M5/3\&5/8 & SIL & $1 \mathrm{Msbk}$ & $\mathrm{Fr}$ & $\mathrm{N}$ & Cs \\
\hline
\end{tabular}




\begin{tabular}{|c|c|c|c|c|c|c|c|c|c|c|c|}
\hline Site & Series & Slope & Aspect & Horizon & Depth & Moist Color & Texture & Structure & Consistency & $\mathbf{P h}$ & Boundary \\
\hline 40 & WAKELAND & 0 & NA & $\mathrm{C}$ & $30-50$ & 10YR6/2 M 5/2\&4/6 & SIL & M & $\mathrm{Fr}$ & $\mathrm{N}$ & Cs \\
\hline 40 & WAKELAND & 0 & NA & C & $50-60$ & 10YR6/1 M 5/2\&4/6 & SIL & M & $\mathrm{Fr}$ & $\mathrm{N}$ & Cs \\
\hline 41 & STOY & 0 & NA & $\mathrm{AP}$ & $0-8$ & 10YR4/2 & SIL & $2 f g r$ & friable & 5.5 & AS \\
\hline 41 & STOY & 0 & NA & $E$ & 8-13. & 10YR5/3 & SIL & 2 fpty & friable & 5.5 & CS \\
\hline 41 & STOY & 0 & NA & BT1 & $13-22$ & $10 \mathrm{yr} 5 / 4, \mathrm{ffd} 5 / 8, \mathrm{ccf} 5 / 3$ & $\mathrm{SICL}$ & $2 m s b k$ & firm & 5 & CS \\
\hline 41 & STOY & 0 & NA & BT2 & $22-30$ & $10 \mathrm{yr} 514, \mathrm{ffd} 5 / 2,5 / 8$ ccf5/1 & $\mathrm{SICL}$ & $2 m s b k$ & firm & 5.5 & CS \\
\hline 41 & STOY & 0 & NA & $\mathrm{BTg} 1$ & $30-42$ & $10 \mathrm{yr} 5 / 2, \mathrm{cfd} 5 / 8,5 / 4 \mathrm{ccf} 5 / 1$ & $\mathrm{SICL}$ & $2 m s b k$ & firm & 6 & CS \\
\hline 41 & STOY & 0 & NA & BTg2 & $42-60$ & $5 / 1 \operatorname{cfd} 5 / 8,5 / 2, \operatorname{ccf} 5 / 1$ & $\mathrm{SICL}$ & $2 m s b k$ & firm & 6 & NA \\
\hline 42 & STOY & 1 & NA & AP & $0-6$ & $10 \mathrm{yr} 5 / 6 \mathrm{cfd} 5 / 1,5 / 8, \mathrm{c} 10 \mathrm{yr} 5 / 2 \mathrm{cf}$ & $\mathrm{SICL}$ & $2 \mathrm{msbk}$ & firm & 5 & AS \\
\hline 42 & STOY & 1 & NA & Bt1 & $6-18$ & $10 \mathrm{yr} 5 / 4, \mathrm{cfd} 5 / 8,5 / 1 \mathrm{cf} 5 / 2$ & $\mathrm{SICL}$ & $2 \mathrm{msbk}$ & firm & 5 & Cs \\
\hline 42 & STOY & 1 & NA & Bt2 & $18-30$ & $10 \mathrm{yr} 5 / 3, \mathrm{cfd} 5 / 8,5 / 1$ c cf $5 / 1$ & $\mathrm{SICL}$ & $2 m s b k$ & firm & 5.5 & Cs \\
\hline 42 & STOY & 1 & NA & $\operatorname{Btg} 1 \mathrm{x}$ & $30-43$ & $10 \mathrm{yr} 5 / 2 \mathrm{cfd} 5 / 8,5 / 4 \mathrm{cf} 5 / 1$ & $\mathrm{SICL}$ & $2 \mathrm{msbk}$ & firm & 6 & Cs \\
\hline 42 & STOY & 1 & NA & Btg2 & $43-60$ & 10yr5/2cfd 10YR5/8\&5/4 dis $5 / 1 \mathrm{cf}$ & NA & NA & NA & NA & NA \\
\hline 43 & $\begin{array}{l}\text { NON-CALCAREOUS } \\
\text { SCHULINE }\end{array}$ & 2 & NW & Ap & $0-8$ & Mixed 10YR4/2\&3/2 & SIL & $1 \mathrm{fgr}$ & $\mathrm{Fr}$ & 6 & As \\
\hline 43 & $\begin{array}{l}\text { NON-CALCAREOUS } \\
\text { SCHULINE }\end{array}$ & 2 & NW & $\mathrm{B} / \mathrm{C} 1$ & 8-19. & mixed10yr5/6,5/1,4/2 cfd 5/8 & SIL\&SICL & $1 \mathrm{msbk}$ & firm & 6 & As \\
\hline 43 & $\begin{array}{l}\text { NON-CALCAREOUS } \\
\text { SCHULINE }\end{array}$ & 2 & NW & $\mathrm{B} / \mathrm{C} 2$ & $19-24$ & Mixed $10 y r 3 / 1,4 / 2$ cfd $5 / 8$ & SIL & $1 \mathrm{fgr}$ & $\mathrm{Fr}$ & 6.5 & As \\
\hline 43 & $\begin{array}{l}\text { NON-CALCAREOUS } \\
\text { SCHULINE }\end{array}$ & 2 & NW & $\mathrm{B} / \mathrm{C} 3$ & 24-39 & mixed $10 y r 5 / 2,5 / 6$ cfd $5 / 8$ & $\mathrm{SICL}$ & CP\&Mass & Vfirm & 7 & As \\
\hline
\end{tabular}




\begin{tabular}{|c|c|c|c|c|c|c|c|c|c|c|c|}
\hline Site & Series & Slope & Aspect & Horizon & Depth & Moist Color & Texture & Structure & Consistency & $\mathbf{P h}$ & Boundary \\
\hline 43 & $\begin{array}{l}\text { NON-CALCAREOUS } \\
\text { SCHULINE }\end{array}$ & 2 & NW & $\mathrm{B} / \mathrm{C} 3$ & $39-60$ & $\operatorname{mix} 10 y r 5 / 2,5 / 6,5 / 1 \mathrm{cfd} 5 / 8$ & CI\&SICL & CP\&Mass & Vfirm & 7 & NA \\
\hline 44 & $\begin{array}{l}\text { NON-CALCAREOUS } \\
\text { SCHULINE }\end{array}$ & 1 & $\mathrm{~N}$ & Ap & $0-11$ & Mixed 10YR3/2\&5/2 & SIL & $1 \mathrm{mgr}$ & $\mathrm{Fr}$ & 5.5 & As \\
\hline 44 & $\begin{array}{l}\text { NON-CALCAREOUS } \\
\text { SCHULINE }\end{array}$ & 1 & $\mathrm{~N}$ & $\mathrm{~B} / \mathrm{C} 1$ & $11-20$ & mixed $10 y r 5 / 6,5 / 1$ and $6 / 2$ & SICL & Mass\&Cp & Vfirm & 5.5 & As \\
\hline 44 & $\begin{array}{l}\text { NON-CALCAREOUS } \\
\text { SCHULINE }\end{array}$ & 1 & $\mathrm{~N}$ & $\mathrm{~B} / \mathrm{C} 2$ & $20-60$ & $\operatorname{mix} 10 y r 4 / 3,5 / 6$ and $5 / 1$ & CL\&SICL & Mass\&Cp & Vfirm & 6.5 & NA \\
\hline 45 & SCHULINE & 2 & $\mathrm{~S}$ & Ap & $0-8$ & $10 \mathrm{yr} 4 / 2 \mathrm{ccf5} / 2, \mathrm{ffd} 10 \mathrm{yr} 5 / 8$ & SIL & $2 \mathrm{fgr}$ & $\mathrm{Fr}$ & 6 & As \\
\hline 45 & SCHULINE & 2 & $S$ & $\mathrm{~B} / \mathrm{C} 1$ & 8-16. & $10 \mathrm{yr} 4 / 2 \mathrm{mmf} 5 / 2$, cfa $5 / 8$ & SIL & $2 m s b k$ & $\mathrm{Fr}$ & 6 & As \\
\hline 45 & SCHULINE & 2 & $S$ & $\mathrm{~B} / \mathrm{C} 2$ & $16-24$ & Mixed 10YR5/1\&5/6 & SIL\&SICL & $\mathrm{CP}$ & Vfirm & 7 & As \\
\hline 45 & SCHULINE & 2 & $\mathrm{~S}$ & $\mathrm{~B} / \mathrm{C} 3$ & $24-50$ & mixed $10 y r 5 / 1,5 / 2,5 / 6$ cfd $5 / 8$ & $\mathrm{CL}$ & $\mathrm{CP}$ & Vfirm & $7.5, \mathrm{FC}$ & As \\
\hline 45 & SCHULINE & 2 & $\mathrm{~S}$ & $\mathrm{~B} / \mathrm{C} 4$ & $50-60$ & Mixed 10YR5/6y & NA & NA & NA & NA & NA \\
\hline 46 & SCHULINE & 2 & $S$ & Ap & $0-4$ & mixed $10 y r 5 / 1,5 / 3$, cfd $5 / 8$ & SIL & $\mathrm{CP}$ & $\mathrm{Fr}$ & 5.5 & As \\
\hline 46 & SCHULINE & 2 & $S$ & $\mathrm{~B} / \mathrm{C} 1$ & $4-10$ & $\operatorname{mix} 10 y r 5 / 1,5 / 6, \operatorname{cfd} 5 / 8$ & SIL & $\mathrm{CP}$ & $\mathrm{Fr}$ & 6 & As \\
\hline 46 & SCHULINE & 2 & $\mathrm{~S}$ & $\mathrm{~B} / \mathrm{C} 2$ & $10-30$ & mixed $10 y r 5 / 4,5 / 2$, cfd $5 / 8$ & $\mathrm{CL}$ & $\mathrm{CP}$ & Vfrim & $7.5 \mathrm{FC}$ & As \\
\hline 46 & SCHULINE & 2 & $\mathrm{~S}$ & $\mathrm{~B} / \mathrm{C} 3$ & $30-60$ & mixed $10 y r 5 / 2,5 / 4 \circ 5 / 6$, cfa $5 / 8$ & $\mathrm{CL}$ & $\mathrm{CP}$ & Vfirm & $7.5, \mathrm{FC}$ & NA \\
\hline 47 & SCHULINE & 0 & NA & Ap & $0-7$ & $10 y r 5 / 1$ cfd $5 / 8$ 4/6 & SIL & $1 \mathrm{Msbk}$ & $\mathrm{Fr}$ & 5.5 & AS \\
\hline 47 & SCHULINE & 0 & NA & $\mathrm{B} / \mathrm{C} 1$ & $7-14$ & mixed 5/1 5/6 5/4 Cmd 5/8 & $\mathrm{CL}$ & $\mathrm{CP}$ & Vfirm & 5.5 & AS \\
\hline 47 & SCHULINE & 0 & NA & $\mathrm{B} / \mathrm{C} 2$ & $14-30$ & Mixed 5/1 6/2 5/4 & CL \& clay & $\mathrm{CP}$ & Vfirm & 7 & AS \\
\hline 47 & SCHULINE & 0 & NA & $\mathrm{B} / \mathrm{C} 3$ & $30-60$ & Mixed 5/6 5/1 5/3 & $\mathrm{CL}$ & $\mathrm{CP}$ & Vfirm & 8 & NA \\
\hline
\end{tabular}




\begin{tabular}{|c|c|c|c|c|c|c|c|c|c|c|c|}
\hline Site & Series & Slope & Aspect & Horizon & Depth & Moist Color & Texture & Structure & Consistency & $\mathbf{P h}$ & Boundary \\
\hline 48 & LENZBURG & 1 & W & AP & $0-7$ & Mixed 5/2 5/4 & SIL \& SICL & $\mathrm{CP}$ & firm & 5.5 & AS \\
\hline 48 & LENZBURG & 1 & W & $\mathrm{B} / \mathrm{C} 1$ & $7-18$ & Mixed 5/2 5//1 5/4 & SICL & $\mathrm{CP}$ & firm & 6.5 & AS \\
\hline 48 & LENZBURG & 1 & W & $\mathrm{B} / \mathrm{C} 2$ & $18-30$ & Mixed 5/2 5/1 5/6 & $\mathrm{CL}$ & $\mathrm{CP}$ & Vfirm & $7.5, \mathrm{FC}$ & AS \\
\hline 49 & LENZBURG & 2 & $E$ & AP & $0-6$ & Mixed 10YR 4/2 5/4 & SIL SILC & $1 \mathrm{Msbk}$ & $\mathrm{fr}$ & 5.5 & AS \\
\hline 49 & LENZBURG & 2 & $E$ & $\mathrm{~B} / \mathrm{C} 1$ & $6-20$ & Mixed ftd 5/6 5/1 5/2 10yr 5r & $\mathrm{CL}$ & $\mathrm{CP}$ & Vfirm & 6.5 & AS \\
\hline 49 & LENZBURG & 2 & $E$ & $\mathrm{~B} / \mathrm{C} 2$ & $20-40$ & Mixed 5/6 5/1 5/8 & $\mathrm{CL}$ & $\mathrm{CP}$ & Vfirm & 7 & AS \\
\hline 51 & BIRDS & 2 & W & AP & $0-7$ & $10 Y R 5 / 2 \mathrm{cfd} 5 / 8$ & SIL & $2 \mathrm{fgr}$ & fr & 5.5 & AS \\
\hline 51 & BIRDS & 2 & W & $\mathrm{Cg} 1$ & $7-14$ & 10YR5/1 cfd 5/8 5/12 & SIL & $\mathrm{M}$ & $\mathrm{fr}$ & 5.5 & CS \\
\hline 51 & BIRDS & 2 & W & $\mathrm{Cg} 2$ & $14-30$ & 10YB6/1 cfd 5/8 5/12 & SIL & $M$ & $\mathrm{fr}$ & 6 & CS \\
\hline 51 & BIRDS & 2 & W & $\mathrm{Cg} 3$ & $30-42$ & 10YR5/1 cfd 5/8 6/1 & SIL & $M$ & $\mathrm{fr}$ & $7.5, \mathrm{FC}$ & CS \\
\hline 51 & BIRDS & 2 & W & $\mathrm{Cg} 4$ & $42-60$ & $2.5 Y 6 / 2 \mathrm{cfd} 5 / 15 / 8$ & SIL & $M$ & $\mathrm{fr}$ & $7.5, \mathrm{FC}$ & CS \\
\hline 53 & SCHULINE & 15 & $E$ & $A$ & $0-7$ & $\operatorname{mix} 10 y r 5 / 2,4 / 2$ & SIL & Ifsbk & $\mathrm{fr}$ & 5.5 & As \\
\hline 53 & SCHULINE & 15 & $E$ & $\mathrm{~B} / \mathrm{C} 1$ & $007-15$ & mixed $10 y r 5 / 2,5 / 6$ & Sil, Sicl & $\mathrm{cp}$ & $\mathrm{fr}$ & 6 & As \\
\hline 53 & SCHULINE & 15 & $E$ & $\mathrm{~B} / \mathrm{C} 2$ & $15-31$ & mixed $10 y r 5 / 6,5 / 3$, cfd $5 / 8$ & $\mathrm{cl} 5 \%$ & $\mathrm{cp}$ & firm & $7.5 \mathrm{FC}$ & As \\
\hline 53 & SCHULINE & 15 & $\mathrm{E}$ & $\mathrm{B} / \mathrm{C} 3$ & $31-60$ & mixed $10 y r 5 / 1,5 / 6,5 / 3$ & $\mathrm{cl} 5 \%$ & $\mathrm{cp}$ & firm & $7.5 \mathrm{FC}$ & NA \\
\hline 55 & LENZBURG & 7 & W & A & $0-11$ & $\operatorname{mix} 5 / 6,5 / 2 \mathrm{cmd} 5 / 8$ & SICL \& CL & $\mathrm{CP}$ & firm & 5.5 & AS \\
\hline 55 & LENZBURG & 7 & W & $\mathrm{B} / \mathrm{C}$ & $11-20$. & Mixed 10YR 5/2 4/1 \& 5/4 cmd 5/8 & CL\&L & $\mathrm{CP}$ & vfirm & 6.5 & AS \\
\hline 55 & LENZBURG & 7 & W & $\mathrm{Cg} 1$ & $20-40$ & $5 Y 5 / 1 \mathrm{cmd} 5 / 8$ 5/4 & $\mathrm{CL}$ & $\mathrm{CP}$ & vfirm & 7.3 & AS \\
\hline
\end{tabular}




\begin{tabular}{|c|c|c|c|c|c|c|c|c|c|c|c|}
\hline Site & Series & Slope & Aspect & Horizon & Depth & Moist Color & Texture & Structure & Consistency & $\mathbf{P h}$ & Boundary \\
\hline 55 & LENZBURG & 7 & W & $\mathrm{Cg} 2$ & $40-60$ & 10YR $6 / 2 \mathrm{cmd} \mathrm{5/8} \mathrm{5/4}$ & $\mathrm{CL}$ & $\mathrm{CP}$ & vfirm & $7.5 \mathrm{FC}$ & NA \\
\hline 56 & SCHULINE & 3 & $E$ & AP & $0-4$ & 10YR 4/2 & SIL & $2 \mathrm{fgr}$ & fr. & 5.5 & AS \\
\hline 56 & SCHULINE & 3 & E & $\mathrm{B} / \mathrm{C} 1$ & $4-7$. & Mixed $10 Y 5$ 5/4 5/6 5/2 & $C L$ & $\mathrm{CP}$ & firm & 5.5 & AS \\
\hline 56 & SCHULINE & 3 & $E$ & $\mathrm{~B} / \mathrm{C} 2$ & $7-22$ & Mixed 10 YR 5/2 5/3 \& 5/4 cfd 5/4 & SIL\&CL & $\mathrm{CP}$ & firm & 6.5 & AS \\
\hline 56 & SCHULINE & 3 & $E$ & $\mathrm{~B} / \mathrm{C} 3$ & $22-40$ & Mixed 10YR 6/2 5/2 \&5/6 & $C L$ & $\mathrm{CP}$ & vfirm & $7.5, \mathrm{FC}$ & AS \\
\hline 56 & SCHULINE & 3 & $E$ & $\mathrm{~B} / \mathrm{C} 4$ & $40-60$ & Mixed 10YR 5/2 6/1 \& 5/6 & $\mathrm{CL}$ & $\mathrm{CP}$ & vfirm & $7.5 \mathrm{FC}$ & NA \\
\hline 57 & SCHULINE & 3 & $E$ & AP & $0-8$ & Mixed 10YR 5/2 5/1, 5/6 & SIL \& SICL & $1 \mathrm{fsbk}$ & $\mathrm{fr}$ & 5.5 & AS \\
\hline 57 & SCHULINE & 3 & $E$ & $\mathrm{~B} / \mathrm{C} 1$ & $8-17$. & Mixed 10YR 5/6 5/2 5/1cfd 5/8 & SICL \& CL & $\mathrm{CP}$ & firm & 6.0 & AS \\
\hline 57 & SCHULINE & 3 & $E$ & $\mathrm{~B} / \mathrm{C} 2$ & $17-30$ & Mixed 10YR 5/6 4/3 5/2cfd 5/8 & SICL \& CL & $\mathrm{CP}$ & firm & 7.5,FC & CS \\
\hline 57 & SCHULINE & 3 & $E$ & $\mathrm{~B} / \mathrm{C} 3$ & $30-60$ & Mixed 10YR 5/6 5/2 5/1cfd 5/8 & $C L$ & $\mathrm{CP}$ & firm & $7.5, \mathrm{FC}$ & $B$ \\
\hline 62 & WAKELAND & 1 & $\mathrm{~N}$ & $A$ & $0-7$ & 10YR 4/3 S & Silt Loam & $2 \mathrm{fgr}$ & $\mathrm{fr}$ & 6.0. & AS \\
\hline 62 & WAKELAND & 1 & $\mathrm{~N}$ & C & 37453 & 10YR 5/3 cff 5/2 5/6 & SIL & M & $\mathrm{fr}$ & 6.0 & CS \\
\hline 62 & WAKELAND & 1 & $\mathrm{~N}$ & C & $16-38$ & 10YR 5/2 cff 5/6 6/4 & SIL & M & $\mathrm{fr}$ & 6.0 & CS \\
\hline 62 & WAKELAND & 1 & $\mathrm{~N}$ & C & $38-60$ & 10YR 5/2 mfd 10yr 5/6 6/1 & SIL & M & $\mathrm{fr}$ & 6.0 . & CS \\
\hline 63 & SCHULINE & 1 & $\mathrm{~N}$ & A & $0-5$ & 10YR41/3 & SIL & Ifq2 & $\mathrm{fr}$ & 5 & AS \\
\hline 63 & SCHULINE & 1 & $\mathrm{~N}$ & $\mathrm{~B} / \mathrm{C}$ & $005-17$ & 10YR414 60YR4/2 & SICL & VCP & EXFI & 6 & ES \\
\hline 63 & SCHULINE & 1 & $\mathrm{~N}$ & C & $17-60$ & N 2/0 10YR4/2 2.5YR4/2 & $\mathrm{CL}$ & $\mathrm{m}$ & vfi & $8, \mathrm{FC}$ & NA \\
\hline 64 & SCHULINE & 1 & $\mathrm{~N}$ & A & $0-3$ & 10YR5/3 & SIL & Ifgr & $\mathrm{fr}$ & 5.5 & AS \\
\hline
\end{tabular}




\begin{tabular}{|c|c|c|c|c|c|c|c|c|c|c|c|}
\hline Site & Series & Slope & Aspect & Horizon & Depth & Moist Color & Texture & Structure & Consistency & $\mathbf{P h}$ & Boundary \\
\hline 64 & SCHULINE & 1 & $\mathrm{~N}$ & $\mathrm{AE}$ & $003-13$ & 10YR5/2 10YR4/6 10YR4/3 & SIL & Ifsbk & $\mathrm{fr}$ & 5.5 & AS \\
\hline 64 & SCHULINE & 1 & $\mathrm{~N}$ & $\mathrm{C} 1$ & $13-33$ & 10YR4/4 5Y511 2.5YR4/2 & SICL & vcp & vfi & 7 & AS \\
\hline 64 & SCHULINE & 1 & $\mathrm{~N}$ & $\mathrm{C} 2$ & $33-60$ & $\mathrm{~N} 2 / 05 \mathrm{Y} 5112.5 \mathrm{Y} 4 / 4$ & $\mathrm{CL}$ & $\mathrm{m}$ & exfi & $8, \mathrm{FC}$ & NA \\
\hline 67 & SCHULINE & 1 & $\mathrm{~N}$ & $A$ & $0-7$ & Mixed 5/2 $1412 \& 516$ & SIL \& CL & $\mathrm{cp}$ & firm & 7 & AS \\
\hline 67 & SCHULINE & 1 & $\mathrm{~N}$ & $\mathrm{~B} / \mathrm{C} 1$ & $007-20$ & mixed $10 Y R 5 / 6 \& 5 / 2$ cfd5/8 & $\mathrm{CL}$ & $\mathrm{cp}$ & firm & 7 & AS \\
\hline 67 & SCHULINE & 1 & $\mathrm{~N}$ & $\mathrm{~B} / \mathrm{C} 2$ & $20-35$ & mixed $5 / 615 / 8 \& 5 / 2$ & SCL\& CL & $\mathrm{cp}$ & firm & 7.5FC & AS \\
\hline 67 & SCHULINE & 1 & $\mathrm{~N}$ & $\mathrm{~B} / \mathrm{C} 2$ & $35-60$ & mixed $5 / 2,5 / 18,5 / 6$ cfd $5 / 18$ & $\mathrm{CL}$ & $\mathrm{cp}$ & firm & $7.5, \mathrm{FC}$ & NA \\
\hline 68 & LENZBURG & 0 & NA & $A$ & $0-10$ & Mixed 5/6,5/2,5/1 cfd. 518 & sil \& cl \% or gr & $\mathrm{cp}$ & firm & 6.6 & AS \\
\hline 68 & LENZBURG & 0 & NA & $\mathrm{B} / \mathrm{C} 1$ & 010-15 & mixed $5 / 2,5 / 6 \& 5 / 1$ & cl 5\%gr & $\mathrm{cp}$ & vfirm & 6.5 & AS \\
\hline 68 & LENZBURG & 0 & NA & $\mathrm{B} / \mathrm{C} 2$ & $15-30$ & mixed $5 / 2,5 / 1 \& 5 / 6$ & $\mathrm{cl}$ & $\mathrm{cp}$ & vfirm & 7 & AS \\
\hline 68 & LENZBURG & 0 & NA & $\mathrm{B} / \mathrm{C} 3$ & $30-60$ & mixed $5 / 3,5 / 2,5 / 1 \& 5 / 6$ & $\mathrm{cl}$ & $\mathrm{cp}$ & vfirm & $7.5 \mathrm{FC}$ & NA \\
\hline 69 & SCHULINE & 15 & $E$ & A & $0-7$ & mixed 10YR 5/2\&4/2 & SIL & Ifsbk & fr & 5.5 & AS \\
\hline 69 & SCHULINE & 15 & $E$ & $\mathrm{~B} / \mathrm{C} 1$ & $007-15$ & mixed 10YR 5/2\&5/6 & Sil \& Sicl & $\mathrm{cp}$ & $\mathrm{fr}$ & 6 & AS \\
\hline 69 & SCHULINE & 15 & $\mathrm{E}$ & $\mathrm{B} / \mathrm{C} 2$ & $15-31$ & mixed $10 Y R 5 / 6 \& 5 / 3$ cfd $5 / 8$ & $\mathrm{cl} 5 \% \mathrm{gf}$ & $\mathrm{cp}$ & firm & $7.5, \mathrm{FC}$ & AS \\
\hline 69 & SCHULINE & 15 & $E$ & $\mathrm{~B} / \mathrm{C} 3$ & $31-60$ & mixed 10YR5/1,5/6,5/3 & $\mathrm{cl} 5 \% \mathrm{gf}$ & $\mathrm{cp}$ & firm & $7.5, \mathrm{FC}$ & NA \\
\hline 72 & BIRDS & 0 & NA & AP & $0-5$ & 10YR5/2 MMf4/2FLD10YR5/8 & Sil & $2 \mathrm{fsbk}$ & $\mathrm{fr}$ & 6.5 & AS \\
\hline 72 & BIRDS & 0 & NA & $\mathrm{Cg} 1$ & $005-18$ & 5YR5/1 cff 10YR6/2\&5/8 & Sil & $1 \mathrm{fsbk}$ & $\mathrm{fr}$ & 6.5 & CS \\
\hline 72 & BIRDS & 0 & NA & $\mathrm{Cg} 2$ & $18-30$ & $5 Y R 5 / 1 \mathrm{cfd} 6 / 1 \& 5 / 8$ & Sil & M & $\mathrm{fr}$ & 7 & CS \\
\hline
\end{tabular}




\begin{tabular}{|c|c|c|c|c|c|c|c|c|c|c|c|}
\hline Site & Series & Slope & Aspect & Horizon & Depth & Moist Color & Texture & Structure & Consistency & $\mathbf{P h}$ & Boundary \\
\hline 72 & BIRDS & 0 & NA & Cg3 & $30-60$ & 2.5YR6/2 cmd 10YR5/2,5/8 & SIL & M & fr & 7 & NA \\
\hline 73 & MORRISTOWN & 0 & NA & $A$ & $0-4$ & 10YR 5/3 & SIL & $1 \mathrm{fgr}$ & fr & 7 & AS \\
\hline 73 & MORRISTOWN & 0 & NA & $\mathrm{B} / \mathrm{C}$ & $004-12$ & mixed 10YR 5/6\&5/2 & CL5\% & M & vfirm & 7 & NA \\
\hline 74 & SCHULINE & 0 & NA & $A$ & $0-3$ & 10YR4/6 10YR 5/4 & SiL & Ifgr & fr & 6 & AS \\
\hline 74 & SCHULINE & 0 & NA & $\mathrm{B} / \mathrm{C}$ & $3-20$. & 10YR4/6 10YR511 10YR4/3 & Sicl & vcp & vfi & 7 & CS \\
\hline 74 & SCHULINE & 0 & NA & $\mathrm{C}$ & $20-60$ & 10YR4/4 M510 2.5Y4/2 & $\mathrm{CL}$ & $\mathrm{m}$ & vfi & $8, \mathrm{FC}$ & NA \\
\hline 75 & SCHULINE & 15 & W & $A B$ & $0-3$ & 10YR4/3 10YR 4/6 & sicl & Ifsbk & fi & 6 & AS \\
\hline 75 & SCHULINE & 15 & W & $\mathrm{BC}$ & 003-12 & 10YR4/3 5Y511 7.5YR 4/6 & sicl & vcp & vfi & $8, \mathrm{FC}$ & AS \\
\hline 75 & SCHULINE & 15 & W & $\mathrm{C}$ & 22251 & $\mathrm{~N} 2 / 0$ N5/0 5Y4/1 7.5YR4/6 & $\mathrm{cl}$ & vcp & vfi & $8, \mathrm{FC}$ & NA \\
\hline 77 & LENZBURG & 15 & W & AP & $0-6$ & mixed 10YR5/6\&5/2 & SICL & $\mathrm{CP}$ & firm & 5.5 & AS \\
\hline 77 & LENZBURG & 15 & W & $\mathrm{B} / \mathrm{C} 1$ & $006-20$ & mixed10YR 5/1\&5/6 cf 5/8 & CL 5\%gr & $\mathrm{CP}$ & vfirm & 6 & AS \\
\hline 77 & LENZBURG & 15 & W & $\mathrm{B} / \mathrm{C} 2$ & $20-42$ & mixed 10YR 5/6 \& 5/1 & cl\&slcl & $\mathrm{CP}$ & firm & 5.5 & AS \\
\hline 77 & LENZBURG & 15 & W & $\mathrm{B} / \mathrm{C} 3$ & $42-60$ & 10YR5/1 cmd 5/8,4/6 & $\mathrm{cl}$ & $\mathrm{CP}$ & firm & $7.5, \mathrm{FC}$ & NA \\
\hline 78 & STOY & 7 & SE & AP & $0-8$ & ff conc. $5 / 2$ & SIL & sil2fgr & fr & 6 & AS \\
\hline 78 & STOY & 7 & SE & Bti & $008-16$ & ffconc.5yr5/4,mfdM5/2cf5/2, 5/8 & SILC & $2 f g b k$ & $\mathrm{fr}$ & 6 & CS \\
\hline 78 & STOY & 7 & SE & Bt2 & $16-30$ & ff.conc. 10YR MMD 5/2 & NA & NA & NA & NA & NA \\
\hline 78 & STOY & 7 & SE & NA & NA & $\& 5 / 8 \mathrm{M} 5 / 2 \mathrm{cf}$ & SICL & $2 f s b k$ & firm & 5.5 & CS \\
\hline 78 & STOY & 7 & SE & Btg1 & $30-40$ & ff.conc. $10 \mathrm{yr} 5 / 2 \mathrm{MMD} 5 / 4,5 / 8 \mathrm{M} / 1 \mathrm{cf}$ & SICL & $2 \mathrm{msbk}$ & firm & 5.5 & CS \\
\hline
\end{tabular}




\begin{tabular}{|c|c|c|c|c|c|c|c|c|c|c|c|}
\hline Site & Series & Slope & Aspect & Horizon & Depth & Moist Color & Texture & Structure & Consistency & $\mathbf{P h}$ & Boundary \\
\hline 78 & STOY & 7 & SE & Btg2 & $40-52$ & ff.conc. $10 \mathrm{yr} 5 / 2 \mathrm{mmd} 5 / 4,5 / 8 \mathrm{~m} 5 / 1 \mathrm{cf}$ & SICL & $2 m s b k$ & firm & 5.5 & CS \\
\hline 78 & STOY & 7 & SE & Btg3 & $52-60$ & ff.conc. $10 \mathrm{yr} 5 / 1 \mathrm{mmd} 5 / 4,5 / 8 \mathrm{~m} 5 / 1 \mathrm{cf}$ & SICL & $2 m s b k$ & firm & 5.5 & CS \\
\hline 79 & STOY & 4 & $S$ & AP & $0-8$ & ff.conc 10YR 4/2 & SIL & $2 \mathrm{fgr}$ & $\mathrm{fr}$ & 6 & AS \\
\hline 79 & STOY & 4 & S & $E$ & 008-14 & ff.conc $5 / 2$ ffd $5 / 8$ & SIL & 2 fpty & $\mathrm{fr}$ & 6 & CS \\
\hline 79 & STOY & 4 & $S$ & $\mathrm{Bt} 1$ & $14-20$ & ff.conc. $10 \mathrm{yr} 5 / 4 \mathrm{cfd} 5 / 2,5 / 8 \mathrm{~m} 5 / 1 \mathrm{cf}$ & SICL & $2 \mathrm{fsbk}$ & firm & 5.5 & CS \\
\hline 79 & STOY & 4 & $\mathrm{~S}$ & Bt2 & $20-32$ & ff.conc. $10 \mathrm{yr}$ mfd5/2,5/8 m 5/2 cf & SICL & $2 \mathrm{fsbk}$ & firm & 5.5 & CS \\
\hline 79 & STOY & 4 & $S$ & $\operatorname{Btg} 1$ & $32-43$ & ff.conc $5 / 2$ mfd $5 / 8 \& 5 / 4 \mathrm{M} 5 / 1 \mathrm{cf}$ & SICL & $2 \mathrm{fsbk}$ & firm & 5.5 & CS \\
\hline 79 & STOY & 4 & $S$ & Btg2 & $43-60$ & ff.conc. 5/1 mfd 5/6\&5/2 M 5/1 CP & Hvy SIL & $2 m s b k$ & $\mathrm{fr}$ & 5.5 & NA \\
\hline 82 & BIRDS & 1 & W & AP & $0-5$ & mised $4 / 3 \& 5 / 2$ & SIL & $1 \mathrm{msbk}$ & $\mathrm{fr}$ & 6 & AS \\
\hline 82 & BIRDS & 1 & W & $\mathrm{B} / \mathrm{C}$ & $005-13$ & mixed 10YR 5/1\&4/3\&5/6 & Slcl 3\% & $\mathrm{cp}$ & firm & 6 & AS \\
\hline 82 & BIRDS & 1 & W & $\mathrm{B} / \mathrm{C}$ & $13-22$ & conc. Mixed 5/1 \&6/2 cpd 5/8 & sil & M & $\mathrm{fr}$ & 6 & AS \\
\hline 82 & BIRDS & 1 & W & $\mathrm{Cg} 1$ & $22-40$ & conc 10YR 5/1 MMD 5\& & sil & M & $\mathrm{fr}$ & 6 & CS \\
\hline 82 & BIRDS & 1 & W & $\mathrm{Cg} 2$ & $40-60$ & 10YR 6/2 MMD 5/8 \& 5/4 & sil & M & $\mathrm{fr}$ & 6 & NA \\
\hline 84 & BIRDS & 0 & NA & $A$ & $0-5$ & 10YR 5/2 & Sil & Mfgz & $\mathrm{fr}$ & 5.5 & AS \\
\hline 84 & BIRDS & 0 & NA & $\mathrm{Bg} 1$ & $005-13$ & 10YR 4/2 & Sil & $2 \mathrm{MPL}$ & $\mathrm{fr}$ & 6 & CS \\
\hline 84 & BIRDS & 0 & NA & $\mathrm{Bg} 2$ & $13-20$ & ED 10YR4/2 CD 10YR5/2 & Sil & $1 \mathrm{msbk}$ & $\mathrm{fr}$ & 6 & CS \\
\hline 84 & BIRDS & 0 & NA & $\mathrm{Bg} 3$ & $20-32$ & 10YR4/6CD 10YA 5/2 & Sil & $1 \mathrm{msbk}$ & $\mathrm{fr}$ & 6.5 & CS \\
\hline 84 & BIRDS & 0 & NA & $\mathrm{Bg} 4$ & $32-60$ & 10YR5/4CD 10YR 5/2 & Sil & $1 \mathrm{msbk}$ & $\mathrm{fr}$ & 7.2 & CS \\
\hline
\end{tabular}




\begin{tabular}{|c|c|c|c|c|c|c|c|c|c|c|c|}
\hline Site & Series & Slope & Aspect & Horizon & Depth & Moist Color & Texture & Structure & Consistency & $\mathbf{P h}$ & Boundary \\
\hline 85 & SCHULINE & 0 & NA & $A B$ & $0-4$ & 10YR4/610YR4/3 & Sicl & Ifsbk & $\mathrm{fi}$ & $7.5, \mathrm{FC}$ & AS \\
\hline 85 & SCHULINE & 0 & NA & $\mathrm{BC}$ & 004-19 & 10YR5/6 10YR4/2 & sicl & vep & $\mathrm{fi}$ & $7.5, \mathrm{FC}$ & CS \\
\hline 85 & SCHULINE & 0 & NA & C & $19-60$ & 5y 5/1 10YR 4/4 2.5 y $4 / 2$ & $\mathrm{CL}$ & M & exfi & $8, \mathrm{FC}$ & NA \\
\hline 86 & SCHULINE & 0 & NA & $A$ & $0-4$ & 10YR4/3 & Sil & Ifsbk & $\mathrm{fr}$ & 6 & AS \\
\hline 86 & SCHULINE & 0 & NA & $A B$ & $004-17$ & 10YR4/3 10YR4/6 & Sicl & $1 \mathrm{msbk}$ & $\mathrm{fi}$ & 6 & AS \\
\hline 86 & SCHULINE & 0 & NA & C & $17-60$ & 5Y5/1 10YR 4/6 & sicl & $\mathrm{vcp}$ & exfi & $8, \mathrm{FC}$ & NA \\
\hline 87 & SCHULINE & 0 & NA & $A B$ & $0-3$ & 10YR 4/4 & Sicl & Imsbk & $\mathrm{fi}$ & 6 & AS \\
\hline 87 & SCHULINE & 0 & NA & $\mathrm{B} / \mathrm{C}$ & $003-18$ & 5YR 5/8 N5/10 7.5YR 5/6 & Sicl & $\mathrm{vcp}$ & vfi & $8, \mathrm{FC}$ & AS \\
\hline 87 & SCHULINE & 0 & NA & C & $18-60$ & 7.5YR5/6 10YR4/2 5Y 4/1 & $\mathrm{CL}$ & $\mathrm{m}$ & exfi & $8, \mathrm{FC}$ & NA \\
\hline 88 & SCHULINE & 0 & NA & A & $0-5$ & 10YR 5/3 & SIL & Ifgv & fv & 6.5 & AS \\
\hline 88 & SCHULINE & 0 & NA & $\mathrm{B} / \mathrm{C} 1$ & $005-17$ & 10YR 4/6 10YR5/4 10YR4/2 & Sicl & $2 \mathrm{mpl} / \mathrm{vco}$ & $\mathrm{FI}$ & $7.5, \mathrm{FC}$ & CS \\
\hline 88 & SCHULINE & 0 & NA & $\mathrm{BC} 2$ & $17-36$ & 7.5YR5/6 10YR4/6 10YR4/2 & sicl & vcp & $\mathrm{FI}$ & 5.5 & CS \\
\hline 88 & SCHULINE & 0 & NA & C & $36-60$ & N 2/0 5 YR5/8 5YR 4/1 & $\mathrm{CL}$ & vcp & $\mathrm{FI}$ & $8, \mathrm{FC}$ & NA \\
\hline 89 & SCHULINE & 0 & NA & A & $0-3$ & conc mixed 10YR 5/6,5/2 & sil \&SICL & $\mathrm{cp}$ & $\mathrm{fr}$ & 5.5 & AS \\
\hline 89 & SCHULINE & 0 & NA & $\mathrm{B} / \mathrm{C} 1$ & 003-11 & conc mixed 10YR 5/6,5/2,5/1 & sil & $\mathrm{cp}$ & firm & 6.5 & AS \\
\hline 89 & SCHULINE & 0 & NA & $\mathrm{B} / \mathrm{C} 2$ & $16-60$ & conc mixed $10 Y R$ 5/6 \& 5/2 & cl $5 \%$ & $\mathrm{cp}$ & firm & 6.5 & AS \\
\hline 90 & MARINE & 1 & $S$ & AP & $0-9$ & f conc. 10YR 4/2 & sil & $2 \mathrm{fgr}$ & $\mathrm{fr}$ & 6 & AS \\
\hline 90 & MARINE & 1 & $\mathrm{~S}$ & $\mathrm{E}$ & 009-16 & f. conc. $10 y r$ r $5 / 2$ ffd $5 / 8$ & sil & $2 \mathrm{fptk}$ & $\mathrm{fr}$ & 6 & CS \\
\hline
\end{tabular}




\begin{tabular}{|c|c|c|c|c|c|c|c|c|c|c|c|c|c|c|}
\hline Site & Series & Slope & Aspect & Horizon & Depth & \multicolumn{4}{|l|}{ Moist Color } & Texture & Structure & Consistency & $\mathbf{P h}$ & Boundary \\
\hline 90 & MARINE & 1 & $S$ & $\mathrm{Bt} 1$ & $16-24$ & \multicolumn{4}{|c|}{ f.conc. $10 Y R 5 / 4 \mathrm{cfd} 5 / 8 \& 5 / 2 \mathrm{~m} 5 / 2 \mathrm{cf}$} & sil & $2 m s b k$ & firm & 6 & CS \\
\hline 90 & MARINE & 1 & $S$ & Bt2 & $24-35$ & \multicolumn{4}{|c|}{$10 \mathrm{yr} 5 / 4 \mathrm{cfd} 5 / 8 \& 5 / 1 \mathrm{M} \mathrm{5/1} \mathrm{cf}$} & sicl & $2 m s b k$ & firm & 5.5 & CS \\
\hline 90 & MARINE & 1 & $S$ & $\mathrm{BTg} 1$ & $35-45$ & \multicolumn{4}{|c|}{ f.conc. $10 \mathrm{yr} 5 / 2$ cfd $5 / 8 \& 5 / 1 \mathrm{~m} 5 / 1 \mathrm{cf}$} & sicl & $2 m s b k$ & firm & 5.5 & CS \\
\hline 90 & MARINE & 1 & $S$ & BTg2 & $45-60$ & \multicolumn{4}{|c|}{ 10yr5/1, mfd 5/8\&5/4 m slict } & sicl & $2 m s b k$ & firm & 5.5 & CS \\
\hline 91 & MARINE & $001 / 2$ & $E$ & AP & $0-8$ & \multicolumn{4}{|c|}{ cf. Conc. 10YR 5/2 } & Sil & 2 fpty & $\mathrm{fr}$ & 6 & AS \\
\hline 91 & MARINE & $001 / 2$ & $E$ & Eg1 & $008-18$ & \multicolumn{4}{|c|}{ cf. Conc. $10 Y$ R5/1 cfd 5/8 } & Sil & 2 fpty & $\mathrm{fr}$ & 6 & CS \\
\hline 91 & MARINE & $001 / 2$ & $E$ & Eg2 & $18-24$ & \multicolumn{4}{|l|}{$\begin{array}{l}\text { cf. Conc. } \\
2.5 \mathrm{yr} 6 / 2\end{array}$} & Sil & 2 fpty & $\mathrm{fr}$ & 6 & CS \\
\hline 91 & MARINE & $001 / 2$ & $E$ & $\operatorname{Btg} 1$ & $24-32$ & \multicolumn{4}{|l|}{$\begin{array}{l}\text { cf. Conc. } \\
5 / 4 \mathrm{~m} 5 / 2 \mathrm{cf}\end{array}$} & Sicl & $2 \mathrm{msbk}$ & firm & 5.5 & CS \\
\hline 91 & MARINE & $001 / 2$ & $E$ & Btg2 & $32-42$ & \multicolumn{4}{|l|}{$\begin{array}{l}\text { cf. Conc. } \\
\text { 5/4M5/1cf }\end{array}$} & Sicl & $2 m s b k$ & firm & 5.5 & CS \\
\hline 91 & MARINE & $001 / 2$ & $E$ & Btg3 & $42-60$ & \multicolumn{4}{|l|}{$\begin{array}{l}\text { cf. Conc. } \\
5 / 2 \mathrm{~m} 5 / 1 \mathrm{cf}\end{array}$} & Sicl & $2 m s b k$ & firm & 5.9 & NA \\
\hline 92 & LENZBURG & 3 & NA & $A$ & $0-4$ & \multicolumn{4}{|c|}{ Mixed $10 Y R 4 / 2 \& 5 / 1$ cfd $10 y r$ 5/8 } & Sil \& Sicl & massive $\mathrm{cp}$ & firm & 6.5 & AS \\
\hline 92 & LENZBURG & 3 & NA & $\mathrm{B} / \mathrm{C} 1$ & $004-15$ & \multicolumn{4}{|c|}{ mixed 10YR5/2 \& 5/6 CFD 10yr5/8 } & $\mathrm{CL}$ & massive $\mathrm{cp}$ & $v$ firm & 7 & AS \\
\hline 92 & LENZBURG & 3 & NA & $\mathrm{B} / \mathrm{C} 2$ & $15-48$ & \multicolumn{4}{|c|}{ mixed $10 Y R 5 / 2,5 / 1 \& 4 / 3$} & sil \& cl & massive $\mathrm{cp}$ & firm & 7 & AS \\
\hline 92 & LENZBURG & 3 & NA & $\mathrm{B} / \mathrm{C} 3$ & $48-60$ & \multicolumn{4}{|c|}{ mixed 5YR5/1\&5/4 cfd 5/8\&4/6 } & cl \& sil & massive $\mathrm{cp}$ & $v$ firm & $7.5, \mathrm{FC}$ & NA \\
\hline 93 & BIRDS & $001 / 3$ & W & AP & $0-7$ & \multicolumn{4}{|l|}{ 10YR5/3 } & sil & $\mathrm{mf} g \mathrm{k}$ & $\mathrm{fr}$ & 5.5 & AS \\
\hline 93 & BIRDS & $001 / 3$ & W & $\mathrm{C} 1$ & $007-16$ & \multicolumn{4}{|c|}{$10 Y R 5 / 2$ cfd $5 / 6 \& 4 / 3$} & sil & M & $\mathrm{fr}$ & 6 & CS \\
\hline 93 & BIRDS & $001 / 3$ & W & $\mathrm{C} 2$ & $16-38$ & \multicolumn{4}{|c|}{ 10YR5/2 mmd 5/6, 4/3 } & sil & M & $\mathrm{fr}$ & 6 & CS \\
\hline
\end{tabular}




\begin{tabular}{|c|c|c|c|c|c|c|c|c|c|c|c|}
\hline Site & Series & Slope & Aspect & Horizon & Depth & Moist Color & Texture & Structure & Consistency & $\mathbf{P h}$ & Boundary \\
\hline 93 & BIRDS & $001 / 3$ & W & $\mathrm{Cg}$ & $38-60$ & 10YR $5 / 1$ mmd 5/6,4/3 & sil & M & $\mathrm{fr}$ & 6 & NA \\
\hline 94 & BIRDS & 0 & NA & $A$ & $0-7$ & conc. 10YR 4/3 & sil & $2 \mathrm{fgr}$ & $\mathrm{fr}$ & 5.5 & CS \\
\hline 94 & BIRDS & 0 & NA & $\mathrm{C}$ & $007-15$ & conc. 10 YR $5 / 3$ cfd $5 / 6,5 / 2$ & sil & M & $\mathrm{fr}$ & 5.5 & CS \\
\hline 94 & BIRDS & 0 & NA & $\mathrm{Cg} 1$ & $15-35$ & conc. 10YR5/2 cmd 5/6,5/4 & sil & M & $\mathrm{fr}$ & 6 & CS \\
\hline 94 & BIRDS & 0 & NA & $\mathrm{Bg} 2$ & $35-60$ & conc. 10YR5/2 mmd 5/6,6/1 & sil & M & $\mathrm{fr}$ & 6 & NA \\
\hline 95 & SCHULINE & 0 & NA & $0-7$ & A & 10YR 5/3 & Sil & $\mathrm{fcp}$ & FR & 7.5,FC & AS \\
\hline 95 & SCHULINE & 0 & NA & $007-34$ & $\mathrm{~B} / \mathrm{C}$ & 10YR4/4, 7/5YR5/6 5YR5/1 & Sicl & $\mathrm{vcp}$ & efi & $8, \mathrm{FC}$ & CS \\
\hline 95 & SCHULINE & 0 & NA & $34-60$ & $\mathrm{C}$ & N2/0 10YR4/1,5/1,5/1 & $\mathrm{CL}$ & $\mathrm{m}$ & efi & $8, \mathrm{FC}$ & NA \\
\hline 96 & SCHULINE & 0 & NA & A & $0-6$ & 10YR 5/3 & sil & $1 \mathrm{fgr}$ & $\mathrm{fr}$ & 7 & AS \\
\hline 96 & SCHULINE & 0 & NA & $\mathrm{BC}$ & $006-15$ & 10YR 4/6 10YR 4/2 & sil & $1 \mathrm{sbk}$ & $\mathrm{fi}$ & $7.5, \mathrm{FC}$ & AS \\
\hline 96 & SCHULINE & 0 & NA & $\mathrm{C} 1$ & $15-30$ & 10YR4/4 n6/0 10YR 4/2 & sicl & $\mathrm{vcp}$ & exfi & $8, \mathrm{FC}$ & GS \\
\hline 96 & SCHULINE & 0 & NA & $\mathrm{C} 2$ & $30-60$ & N2/0 N 5/0 10YR 4/4 & $\mathrm{cl}$ & $\mathrm{vcp}$ & exfi & $8, \mathrm{FC}$ & NA \\
\hline 97 & SCHULINE & 0 & NA & A & $0-5$ & 10YR 5/3 & sil & $1 \mathrm{fgr}$ & $\mathrm{fr}$ & 6.5 & AS \\
\hline 97 & SCHULINE & 0 & NA & $\mathrm{B} / \mathrm{C} 1$ & $005-13$ & 10YR4/4 5YR 5/1 & sicl & $\mathrm{vcp}$ & exfi & $8, \mathrm{FC}$ & CS \\
\hline 97 & SCHULINE & 0 & NA & $\mathrm{B} / \mathrm{C} 2$ & $13-28$ & 10YR 6/ 5YR 5/1 & sicl & $\mathrm{vcp}$ & exfi & 3.5 & CS \\
\hline 97 & SCHULINE & 0 & NA & $\mathrm{B} / \mathrm{C} 3$ & $28-42$ & 5YR 5/8 5YR 5/1 & sicl & $\mathrm{vcp}$ & exfi & $8, \mathrm{FC}$ & CS \\
\hline 97 & SCHULINE & 0 & NA & $\mathrm{B} / \mathrm{C} 4$ & $42-60$ & 10YR4/4 5YR 5/1 & $\mathrm{cl}$ & $\mathrm{m}$ & exfi & $8, \mathrm{FC}$ & NA \\
\hline 101 & LENZBURG & 15 & NA & C & $0-60$ & calc 10 YR $5 / 6,5 / 2$ & loam,cl till & $\mathrm{cp}$ & vfirm & 7.5,FC & NA \\
\hline
\end{tabular}




\begin{tabular}{|c|c|c|c|c|c|c|c|c|c|c|c|}
\hline Site & Series & Slope & Aspect & Horizon & Depth & Moist Color & Texture & Structure & Consistency & $\mathbf{P h}$ & Boundary \\
\hline 102 & MARINE & 0 & NA & AP & $0-8$ & f. conc. $5 / 2$ & sil & $2 \mathrm{fgr}$ & $\mathrm{fr}$ & 6 & AS \\
\hline 102 & MARINE & 0 & NA & AP & $008-13$ & f. conc. $5 / 2$ cf $5 / 8$ & sil & 2fpty & $\mathrm{fr}$ & 6 & CS \\
\hline 102 & MARINE & 0 & NA & AP & $13-20$ & f. conc. $5 / 6$ cf $5 / 8 \& 5 / 2 \mathrm{~m} 5 / 2 \mathrm{cf}$ & sicl & $2 \mathrm{fpsbk}$ & firm & 6 & CS \\
\hline 102 & MARINE & 0 & NA & AP & $20-32$ & f. conc. $5 / 6 \mathrm{cf} 5 / 8,5 / 2 \mathrm{~m} \mathrm{5/2} \mathrm{cf}$ & sicl & $2 \mathrm{fsbk}$ & firm & 6 & CS \\
\hline 102 & MARINE & 0 & NA & AP & $32-45$ & f. conc. $5 / 2$ cf $5 / 8,5 / 3 \mathrm{~m} 5 / 1 \mathrm{cf}$ & sicl & $2 \mathrm{fsbk}$ & firm & 5.5 & CS \\
\hline 102 & MARINE & 0 & NA & AP & $45-60$ & f. conc. $5 / 1 \mathrm{cf} 5 / 8,5 / 3 \mathrm{~m} \mathrm{5/1} \mathrm{cf}$ & sil & $2 \mathrm{fsbk}$ & fria & 5.5 & NA \\
\hline 103 & MARINE & 0 & NA & AP & $0-8$ & f.conc. $5 / 2$ & sil & 2 fgy & $\mathrm{fr}$ & 6 & AS \\
\hline 103 & MARINE & 0 & NA & $E$ & $008-12$ & f.conc. Crd 5/6 & sil & 2 fgty & $\mathrm{fr}$ & 6 & AS \\
\hline 103 & MARINE & 0 & NA & Bt1 & 37620 & f. conc.10YR5/6 ffd5/8, 5/2 m5/2cf & sicl & $2 \mathrm{fsbk}$ & $\mathrm{fr}$ & 5.5 & cs \\
\hline 103 & MARINE & 0 & NA & $\mathrm{Bt} 2$ & $30-39$ & f. conc.10YR5/4 ffd 5/2,5/6 m5/2cf & sicl & $2 \mathrm{msbk}$ & firm & 5.5 & CS \\
\hline 103 & MARINE & 0 & NA & $\mathrm{Btg} 1$ & $39-50$ & $10 \mathrm{yr} 5 / 2 \mathrm{cfd} 5 / 8,3 / 7 \mathrm{Md} 5 / 6 \mathrm{ct}$ & sicl & $2 \mathrm{msbk}$ & firm & 5.5 & NA \\
\hline 103 & MARINE & 0 & NA & $\mathrm{B} / \mathrm{C}$ & $50-60$ & 10YR 5/1 cfd 5/8 MD3/1 cfd & NA & NA & NA & NA & NA \\
\hline 104 & BIRDS & 0 & NA & $A$ & $0-8$ & $10 Y R 5 / 2 \mathrm{ffd} 5 / 8$ & Sil & mmgr & $\mathrm{fr}$ & 6 & NA \\
\hline 104 & BIRDS & 0 & NA & $\mathrm{Cg} 1$ & $008-16$ & 10YR5/2mmd 5/8\&4/6 & sil & $\mathrm{m}$ & $\mathrm{fr}$ & 6 & NA \\
\hline 104 & BIRDS & 0 & NA & $\mathrm{Cg} 2$ & $16-30$ & 10YR5/1 mmd 5/8,4/6 & sil & $\mathrm{m}$ & $\mathrm{fr}$ & 6 & NA \\
\hline 104 & BIRDS & 0 & NA & Cg3 & $30-60$ & $2.5 \mathrm{YR} 6 / 2 \mathrm{mmd} 5 / 8,4 / 6$ & sil & $\mathrm{m}$ & $\mathrm{fr}$ & 6 & NA \\
\hline 105 & WAKELAND & 0 & NA & $A$ & $0-8$ & conc. $10 \mathrm{YR} 4 / 3$ & sil & $2 \mathrm{fgr}$ & $\mathrm{fr}$ & 5.5 & AS \\
\hline 105 & WAKELAND & 0 & NA & $\mathrm{C} 1$ & 008-17 & conc. $5 / 3$ fff $5 / 2$ & sil & $\mathrm{n}$ & $\mathrm{fr}$ & 5.5 & CS \\
\hline
\end{tabular}




\begin{tabular}{|c|c|c|c|c|c|c|c|c|c|c|c|}
\hline Site & Series & Slope & Aspect & Horizon & Depth & Moist Color & Texture & Structure & Consistency & $\mathbf{P h}$ & Boundary \\
\hline 105 & WAKELAND & 0 & NA & $\mathrm{Cg} 1$ & $17-29$ & conc. $5 / 2$ cmd 5/6\&6/1 & sil & $\mathrm{n}$ & $\mathrm{fr}$ & 5.5 & CS \\
\hline 105 & WAKELAND & 0 & NA & $\mathrm{Cg} 2$ & $29-60$ & conc. $5 / 1$ cmd 5/6, 5/2, 6/1 & sil & $\mathrm{n}$ & $\mathrm{fr}$ & 5.5 & NA \\
\hline 106 & LENZBURG & 0 & NA & $A$ & $0-6$ & 10YR 4/6 10YR 5/3 & sil & $1 \mathrm{fgr}$ & $\mathrm{fr}$ & 6 & AS \\
\hline 106 & LENZBURG & 0 & NA & $\mathrm{BC}$ & 006-18 & 10YR4/3 5YR5/8 2.5YR4/2 & sicl & vcp & vfl & $8, \mathrm{FC}$ & AS \\
\hline 106 & LENZBURG & 0 & NA & & $18-60$ & N2/0 5YR5/1 10YR4/3 & $\mathrm{cl}$ & $\mathrm{m}$ & exfi & $8, \mathrm{FC}$ & NA \\
\hline 107 & SCHULINE & 0 & NA & $A$ & $0-5$ & 10YR 3/3 & sil & $1 \mathrm{fgr}$ & $\mathrm{fr}$ & 6 & AS \\
\hline 107 & SCHULINE & 0 & NA & $A / E$ & $005-13$ & 10YR 5/4 10YR 5/2 & sil & $1 \mathrm{fgr}$ & $\mathrm{fr}$ & 7 & CS \\
\hline 107 & SCHULINE & 0 & NA & $\mathrm{B} / \mathrm{C}$ & $13-24$ & 10YR5/1,10YR5/4, 2.5YR 4/2 & sicl & vcp & vfi & $8, \mathrm{FC}$ & CS \\
\hline 107 & SCHULINE & 0 & NA & C & $24-60$ & 7.5YR4/6 2.5YR4/2 5YR 5/1 & $\mathrm{cl}$ & $\mathrm{m}$ & VFI & $8, \mathrm{FC}$ & NA \\
\hline 108 & SCHULINE & 0 & NA & $A$ & $0-4$ & 10YR 5/3 & sil & $1 \mathrm{fgr}$ & $\mathrm{fr}$ & 6 & AS \\
\hline 108 & SCHULINE & 0 & NA & $A / B$ & 004-14 & 10YR 4/6 10YR 5/3 & sil & $1 \mathrm{fsbk}, 1 \mathrm{fgv}$ & $\mathrm{fr}$ & 6.5 & AS \\
\hline 108 & SCHULINE & 0 & NA & $B / C$ & $14-28$ & 5YR5/1 5YR5/6 2.5YR 4/2 & sicl & vcp & vfi & $8, \mathrm{FC}$ & GS \\
\hline 108 & SCHULINE & 0 & NA & C & $28-60$ & N2/0 10YR5/8 5YR 5/1 & $\mathrm{cl}$ & vcp & esfi & $8, \mathrm{FC}$ & NA \\
\hline 110 & SCHULINE & 0 & NA & $A$ & $0-4$ & 10YR 5/3 & sil & $2 f g v$ & $\mathrm{fv}$ & 6 & CS \\
\hline 110 & SCHULINE & 0 & NA & $\mathrm{A} / \mathrm{E}$ & 004-17 & $10 \mathrm{yr} 4 / 6,10 \mathrm{yr} 5 / 6,10 \mathrm{yr} 5 / 3$ & sicl & $1 \mathrm{fg} v$ & $\mathrm{fv}$ & 7 & AS \\
\hline 110 & SCHULINE & 0 & NA & $B / C$ & $17-29$ & $10 \mathrm{yr} 4 / 4,10 \mathrm{yr} 4 / 1,10 \mathrm{yr} 5 / 6$ & sicl & vcp & vfi & $8, \mathrm{FC}$ & AS \\
\hline 110 & SCHULINE & 0 & NA & C & $29-45$ & $\mathrm{~N} 2 / 07.5 \mathrm{yr} 4 / 6, \mathrm{~N} 5 / 1 \mathrm{coal}$ & sicl & $\mathrm{m}$ & exfi & $8, \mathrm{FC}$ & NA \\
\hline 111 & SCHULINE & 0 & NA & A & $0-8$ & 10YR 5/3 & sil & $1 \mathrm{fgr}$ & $\mathrm{fr}$ & 6.5 & AS \\
\hline
\end{tabular}




\begin{tabular}{|c|c|c|c|c|c|c|c|c|c|c|c|}
\hline Site & Series & Slope & Aspect & Horizon & Depth & Moist Color & Texture & Structure & Consistency & $\mathbf{P h}$ & Boundary \\
\hline 111 & SCHULINE & 0 & NA & $A / B$ & $008-25$ & $10 \mathrm{yr} 4 / 4,7.5 \mathrm{yr} 4 / 6,10 \mathrm{yr} 5 / 2$ & sil & $1 \mathrm{mpl} / \mathrm{cp}$ & $f v$ & 7 & CS \\
\hline 111 & SCHULINE & 0 & NA & $\mathrm{B} / \mathrm{C}$ & $25-46$ & $10 \mathrm{yr} 5 / 6,10 \mathrm{yr} 4 / 4,10 \mathrm{yr} 4 / 2$ & sicl & vcp & exfi & $8, \mathrm{FC}$ & AS \\
\hline 111 & SCHULINE & 0 & NA & C & $46-50$ & $5 G 5 / 17.5 y r 4 / 6$ & sicl & $\mathrm{vcp} / \mathrm{m}$ & exfi & $8, \mathrm{FC}$ & NA \\
\hline 112 & SCHULINE & 0 & NA & A & $0-9$ & $10 y r 5 / 2 \quad 10 y r 5 / 3$ & sil & $1 \mathrm{fgr}$ & $\mathrm{fr}$ & 6 & AS \\
\hline 112 & SCHULINE & 0 & NA & $\mathrm{A} / \mathrm{E}$ & $009-14$ & $10 y r 4 / 6 \quad 10 y r 4 / 3$ & sil & $1 \mathrm{fgr}$ & $\mathrm{fr}$ & 6 & AS \\
\hline 112 & SCHULINE & 0 & NA & $\mathrm{B} / \mathrm{C} 1$ & $14-48$ & $10 y r 5 / 17.5 y r 5 / 110 y r 5 / 4$ & sicl & $\mathrm{vcp}$ & vfi & $8, \mathrm{FC}$ & CS \\
\hline 112 & SCHULINE & 0 & NA & $\mathrm{B} / \mathrm{C} 2$ & $48-60$ & 7.5yr4/6, 10yr5/4 5B 5/1 & $\mathrm{cl}$ & $\mathrm{vcp}$ & exfi & $8, \mathrm{FC}$ & \\
\hline 113 & SCHULINE & 0 & NA & A & $0-7$ & mixed $10 y r 5 / 6 \& 5 / 2$ & $\mathrm{cl} 5 \%$ & $1 \mathrm{msbk}$ & firm & 7 & AS \\
\hline 113 & SCHULINE & 0 & NA & $\mathrm{B} / \mathrm{C} 1$ & $007-18$ & mixed $10 y r 5 / 2 \& 5 / 6$ & $\mathrm{cl} 5 \%$ & $\mathrm{cp}$ & firm & $7.5, \mathrm{FC}$ & AS \\
\hline 113 & LENZBURG & 0 & NA & $\mathrm{B} / \mathrm{C} 2$ & $18-60$ & mixed $10 y r 5 / 6,5 / 8,5 / 2$ & cl $5 \%$ & $\mathrm{cp}$ & $v$ firm & $7.5, \mathrm{FC}$ & NA \\
\hline 114 & STOY & 0 & NA & AP & $0-8$ & cfconc. $5 / 2$ fififout $5 / 3$ & SIL & $2 \mathrm{fgr}$ & $\mathrm{fr}$ & 6 & AS \\
\hline 114 & STOY & 0 & NA & $E$ & $008-13$ & cfconc. $5 / 2$ cff $6 / 2 \& 5 / 4$ & SIL & 2fpty & $\mathrm{fr}$ & 5.5 & CS \\
\hline 114 & STOY & 0 & NA & Bt1 & $13-22$ & $\begin{array}{l}\text { cfconc. } 10 y r \text { 5/4, cfd } 5 / 2 \& 5 / 8 \mathrm{M} 10 y r \\
5 / 2 \text { cf \& } 7 / 2 \text { silt coats }\end{array}$ & SIL & $2 m s b k$ & firm & 5.5 & CS \\
\hline 114 & STOY & 0 & NA & Bt2 & $22-40$ & $\begin{array}{l}10 y r 3 / 4 \text { cfd } 5 / 2 \quad \& 5 / 8 \quad M 5 / 2 c f \quad f 7 / 2 \\
\text { s.c. }\end{array}$ & hvy SICL & $2 m s b k$ & firm & 5.5 & CS \\
\hline 114 & STOY & 0 & NA & Btg1 & $40-48$ & $10 y r 5 / 2$ cfd $5 / 2$ cf $5 / 8 \& 5 / 1$ & SICL & $2 m s b k$ & firm & 5.5 & CS \\
\hline 114 & STOY & 0 & NA & Btg2 & $48-60$ & $10 \mathrm{yr} 5 / 1 \mathrm{cfd} 6 / 8 \& 5 / 4$ f $5 / 2 \mathrm{cf}$ & SICL & $1 \mathrm{msbk}$ & firm & 6 & NA \\
\hline 115 & STOY & 1 & NA & AP & $0-8$ & f. conc. $10 Y R 5 / 2$ & sil & $1 \mathrm{fgr}$ & $\mathrm{fr}$ & 6 & AS \\
\hline 115 & STOY & 1 & NA & $E$ & $008-12$ & $10 y r 5 / 3$ cff $5 / 2$ & sil & 2 fpty & $\mathrm{fr}$ & 6 & CS \\
\hline
\end{tabular}




\begin{tabular}{|c|c|c|c|c|c|c|c|c|c|c|c|}
\hline Site & Series & Slope & Aspect & Horizon & Depth & Moist Color & Texture & Structure & Consistency & $\mathrm{Ph}$ & Boundary \\
\hline 115 & STOY & 1 & NA & Bt1 & $012-20$ & f.conc. $10 \mathrm{yr} 5 / 4 \mathrm{cff} 5 / 8 \mathrm{M} 5 / 2,5 / 3 \mathrm{cf}$ & sicl & $2 f s b k$ & fr & 5.5 & CS \\
\hline 115 & STOY & 1 & NA & Bt2 & $20-35$ & 10yr5/4 Mff5/8 \& 5/1 M5/1 cf & sicl & $2 \mathrm{msbk}$ & firm & 5.5 & CS \\
\hline 115 & STOY & 1 & NA & Btg1 & $35-49$ & f. conc. $2.5 \mathrm{yr} 6 / 2 \mathrm{cfd} 5 / 8 \& 5 / 45 / 1 \mathrm{cf}$ & sicl & $2 \mathrm{msbk}$ & frim & 5.5 & CS \\
\hline 115 & STOY & 1 & NA & Btg2 & $49-60$ & $5 Y r 11$ Cfd5/8\&5/3 C 5/1 cf & sil & $2 \mathrm{msbk}$ & $\mathrm{fr}$ & 5.5 & CS \\
\hline 116 & BIRDS & 0 & NA & A & $0-7$ & f. conc. $5 \mathrm{Yr} 5 / 1 \mathrm{cfd} 5 / 8 \quad 3 / 1$ & sil & $1 \mathrm{fsbk}$ & fr & 6 & CS \\
\hline 116 & BIRDS & 0 & NA & $\mathrm{C} 1$ & $007-17$ & f.conc. $5 y r 5 / 1, \operatorname{mfd} 5 / 8,4 / 2$ & sil & $\mathrm{m}$ & $\mathrm{fr}$ & 6 & CS \\
\hline 116 & BIRDS & 0 & NA & $\mathrm{C} 2$ & $17-35$ & f.conc. $2.5 \mathrm{yr} 6 / 2 \mathrm{mfd} 5 / 8 \& 5 / 2$ & sil & $\mathrm{m}$ & $\mathrm{fr}$ & 6 & CS \\
\hline 116 & BIRDS & 0 & NA & C3 & $35-40$ & f.conc. $2.5 \mathrm{yr} 6 / 2 \mathrm{mmd} 5 / 8$ & sil & $\mathrm{m}$ & fr & 6 & NA \\
\hline 117 & SCHULINE & 0 & NA & A & $0-10$ & $10 \mathrm{Yr} 4 / 3$ & sil & $1 \mathrm{fgr}$ & $\mathrm{fr}$ & 6 & AS \\
\hline 117 & SCHULINE & 0 & NA & $\mathrm{B} / \mathrm{C} 1$ & 010-15 & $10 y r 7 / 410 y r 5 / 6$ & $\mathrm{sl}$ & $1 \mathrm{fsbk}$ & fr & 7 & CS \\
\hline 117 & SCHULINE & 0 & NA & $\mathrm{B} / \mathrm{C} 2$ & $15-24$ & $10 y r 5 / 4,10 y r 5 / 6,10 y r 4 / 2$ & $\mathrm{cl}$ & vcp & exfi & $8, \mathrm{FC}$ & CS \\
\hline 117 & SCHULINE & 0 & NA & $\mathrm{B} / \mathrm{C} 3$ & $24-60$ & $2.5 y r 4 / 110 y r 4 / 2$ & $\mathrm{cl}$ & $\mathrm{m}$ & exfi & $8, \mathrm{FC}$ & NA \\
\hline 118 & SCHULINE & 0 & NA & $A$ & $0-4$ & $10 y r 5 / 3$ & sil & $1 \mathrm{fgl}$ & $\mathrm{fr}$ & 6 & AS \\
\hline 118 & SCHULINE & 0 & NA & $\mathrm{BC}$ & $004-15$ & $7.5 y r$ 4/4 10yr 4/2 & sicl & vcp & vfi & $8, \mathrm{FC}$ & AS \\
\hline 118 & SCHULINE & 0 & NA & C & $15-60$ & $5 y r 5 / 17.5 y r 4 / 6,10 y r 5 / 6$ & $\mathrm{cl}$ & $\mathrm{m}$ & vfi & $8, \mathrm{FC}$ & NA \\
\hline 119 & SCHULINE & 0 & NA & $A$ & $0-10$ & $10 y r 5 / 3$ & sil & $1 \mathrm{fgr}$ & $\mathrm{fr}$ & 6 & AS \\
\hline 119 & SCHULINE & 0 & NA & $\mathrm{B} / \mathrm{C} 1$ & 010-15 & $10 y r 5 / 410 y r 5 / 2$ & sicl & vcp & $\mathrm{fi}$ & $8, \mathrm{FC}$ & CS \\
\hline 119 & SCHULINE & 0 & NA & $\mathrm{B} / \mathrm{C} 2$ & $15-25$ & $10 \mathrm{yr} 5 / 8,10 \mathrm{yr} 5 / 1,10 \mathrm{yr} 5 / 6$ & sicl & vcp & vfi & $8, \mathrm{FC}$ & CS \\
\hline
\end{tabular}




\begin{tabular}{|c|c|c|c|c|c|c|c|c|c|c|c|}
\hline Site & Series & Slope & Aspect & Horizon & Depth & Moist Color & Texture & Structure & Consistency & $\mathbf{P h}$ & Boundary \\
\hline 119 & SCHULINE & 0 & NA & $\mathrm{B} / \mathrm{C} 3$ & $25-29$ & $7.5 \mathrm{yr} 4 / 6,10 \mathrm{yr} 5 / 6,5 \mathrm{yr} 5 / 1$ & I & vcp & $\mathrm{fi}$ & 3.5 & CS \\
\hline 119 & SCHULINE & 0 & NA & $\mathrm{B} / \mathrm{C} 4$ & $29-60$ & 7.5yr4/6 10yr5/4 10yr5/1 & $\mathrm{cl}$ & vcp & exfi & $8, \mathrm{FC}$ & NA \\
\hline 121 & LENZBURG & 10 & NA & A & $0-6$ & mixed $10 y r$ 5/6, 5/2 & $\mathrm{cl} 5 \%$ & $1 \mathrm{msbk}$ & firm & 7 & AS \\
\hline 121 & LENZBURG & 10 & NA & $\mathrm{B} / \mathrm{C} 1$ & $007-18$ & mixed $10 y r 5 / 2,5 / 6$ & $\mathrm{cl} 5 \%$ & NA & firm & $7.5, \mathrm{FC}$ & AS \\
\hline 121 & LENZBURG & 10 & NA & $\mathrm{B} / \mathrm{C} 2$ & $18-60$ & mixed $10 y r 5 / 6,5 / 8,5 / 2$ & $\mathrm{cl} 5 \%$ & NA & $v$ firm & $7.5, \mathrm{FC}$ & NA \\
\hline 122 & LENZBURG & 1 & NA & A & $0-8$ & mixed $10 y r 5 / 6,4 / 2,5 / 1,5 \%$ & cl, sicl, sil & $1 \mathrm{msbk}$ & firm & 6 & AS \\
\hline 122 & LENZBURG & 1 & NA & $\mathrm{B} / \mathrm{C} 1$ & $008-23$ & mixed $5 y r 5 / 1,10 y r 5 / 6,6 / 35 \%$ & cl\&sicl & $\mathrm{cp}$ & firm & $7.5, \mathrm{FC}$ & AS \\
\hline 122 & LENZBURG & 1 & NA & $\mathrm{B} / \mathrm{C} 2$ & $23-49$ & mixed $5 y r 5 / 1,10 y r 5 / 3,5 / 65 \%$ & $\mathrm{cl}$ & $\mathrm{cp}$ & $v$ firm & $7.5, \mathrm{FC}$ & AS \\
\hline 122 & LENZBURG & 1 & NA & $\mathrm{B} / \mathrm{C} 3$ & $49-60$ & mixed $5 y r 5 / 1,5 / 3,5 / 6,5-10 \%$ coal & $\mathrm{cl}$ & $\mathrm{cp}$ & $v$ firm & $7.5, \mathrm{FC}$ & NA \\
\hline 123 & na & NA & NA & NA & NA & $\begin{array}{l}\text { Middle of Rock Spill way } 2 \mathrm{ft} \text { of RR5 } \\
\text { rip rap }\end{array}$ & NA & NA & NA & NA & NA \\
\hline 124 & HOSMER & 3 & W & AP & $0-6$ & $10 y r 4 / 3$ & sil & $2 \mathrm{fsbk}$ & $\mathrm{fr}$ & 6.5 & AS \\
\hline 124 & HOSMER & 3 & W & Bt1 & $006-12$ & $10 \mathrm{yr} 5 / 6,4 / 4 \mathrm{cf}$ cfd $10 \mathrm{yr} 5 / 2$ & sicl & $2 m s b k$ & firm & 6 & CS \\
\hline 124 & HOSMER & 3 & W & Bt2 & $012-20$ & $10 \mathrm{yr} 5 / 4 \mathrm{c} 5 / 2 \mathrm{cf} \& 5 / 2,5 / 3$ & sicl & $2 m s b k$ & firm & 5.5 & CS \\
\hline 124 & HOSMER & 3 & W & Bt2 & $20-28$ & $10 \mathrm{yr} 5 / 4,10 \mathrm{yr} 5 / 2 \mathrm{cf} \mathrm{md} 5 / 8$ & sicl & $2 m s b k$ & firm & 5.5 & CS \\
\hline 124 & HOSMER & 3 & W & $\mathrm{Bt3}$ & $28-45$ & $10 y r 5 / 2$ mld. $5 / 8 \& 4 / 6$ & sicl & $2 m s b k$ & $\mathrm{fr}$ & 5.5 & CS \\
\hline 124 & HOSMER & 3 & W & $\mathrm{BC}$ & $45-60$ & $10 y r 5 / 2 m i d 10 y r 5 / 8,4 / 6$ & sil & $2 m s b k$ & fr & 5.5 & CS \\
\hline 125 & STOY & 0 & NA & AP & $0-7$ & $10 y r 4 / 7$ & sil & $2 \mathrm{fgr}$ & $\mathrm{fr}$ & 6 & As \\
\hline 125 & STOY & 0 & NA & Bt1 & $007-16$ & $10 \mathrm{yr} 5 / 4 \mathrm{cfd} 5 / 8 \& 5 / 25 / 3 \mathrm{cf}$ & sicl & $2 m s b k$ & $\mathrm{fr}$ & 6 & Cs \\
\hline
\end{tabular}




\begin{tabular}{|c|c|c|c|c|c|c|c|c|c|c|c|}
\hline Site & Series & Slope & Aspect & Horizon & Depth & Moist Color & Texture & Structure & Consistency & $\mathbf{P h}$ & Boundary \\
\hline 125 & STOY & 0 & NA & $\operatorname{Btg} 1$ & $16-26$ & $10 \mathrm{yr} 5 / 2 \mathrm{cfd} 5 / 8,5 / 4 \mathrm{C} 5 / 1 \mathrm{cf}$ & sicl & $2 m s b k$ & firm & 7 & Cs \\
\hline 125 & STOY & 0 & NA & Btg2 & $26-32$ & $10 \mathrm{yr} 5 / 1 \mathrm{cfd} 5 / 8 \& 5 / 4$ c 5/1 cf & sicl & $2 m s b k$ & firm & 7 & Cs \\
\hline 125 & STOY & 0 & NA & Btg3 & $32-47$ & f.corc. $5 / 1 \mathrm{ffd} 5 / 8 \& 4 / 6$ cf $5 / 1$ & sicl & $2 m s b k$ & firm & 7.5 & Cs \\
\hline 125 & STOY & 0 & NA & Btg 4 & $47-60$ & f.corc. $5 / 1 \mathrm{ffd} 5 / 8 \& 4 / 6 \mathrm{cf} 5 / 1$ & sil & $2 m s b k$ & firm & 7.5 & Cs \\
\hline 126 & STOY & 0 & NA & AP & $0-9$ & f. conc. $5 / 2$ & sil & $2 \mathrm{fgr}$ & $\mathrm{fr}$ & 6 & AS \\
\hline 126 & STOY & 0 & NA & $\mathrm{Bt} 1$ & $009-17$ & $10 y r 5 / 3$ cff $5 / 2,5 / 6$ CF $5 / 1$ & sicl & $2 m s b k$ & firm & 5.5 & CS \\
\hline 126 & STOY & 0 & NA & $\operatorname{Btg} 1$ & $17-28$ & $10 y r 5 / 2$, cfd $5 / 4,5 / 6$ cf $5 / 1$ & sicl & $2 m s b k$ & firm & 5.5 & CS \\
\hline 126 & STOY & 0 & NA & Btg2 & $28-40$ & $10 \mathrm{yr} 5 / 1$ cfd $5 / 8,4 / 6$ cf $5 / 1$ & sicl & $2 m s b k$ & firm & 5.5 & CS \\
\hline 126 & STOY & 0 & NA & Btg3 & $40-60$ & $10 y r 5 / 1 \mathrm{M} 5 / 8$ cf $5 / 1$ & sicl & $2 m s b k$ & firm & 6 & NA \\
\hline 127 & Good Oconee & $001 / 2$ & $S$ & $A$ & $0-9$ & con. 10yr 3/1 & sil & 2 for & $\mathrm{fr}$ & 6 & As \\
\hline 127 & Good Oconee & $001 / 2$ & $S$ & E1 & $009-13$ & con. $10 y r 5 / 2$ com $3 / 1$ oc & sil & 2 fpty & $\mathrm{fr}$ & 5.5 & Cs \\
\hline 127 & Good Oconee & $001 / 2$ & $S$ & E2 & $13-18$ & conc. $10 \mathrm{yr} 5 / 1 \mathrm{ffd} 10 \mathrm{yr} 5 / 8$ & sil & 2 fpty & firm & 5.5 & Cs \\
\hline 127 & Good Oconee & $001 / 2$ & $S$ & $\mathrm{Bt} 1$ & $18-27$ & $10 \mathrm{yr} 5 / 4 \mathrm{crd} 5 / 2,5 / 8 \quad 5 / 1 \mathrm{cf}$ conc & sicl & $2 m s b k$ & firm & 5.5 & Cs \\
\hline 127 & Good Oconee & $001 / 2$ & $S$ & Btg1 & $27-40$ & $10 y r 5 / 1$ m 10yr5/8,1/6 & sicl & $2 m s k k$ & firm & 5.5 & Cs \\
\hline 127 & Good Oconee & $001 / 2$ & $\mathrm{~S}$ & Btg2 & $40-60$ & $5 y r 5 / 1 \mathrm{ffd} 5 / 8 \& 4 / 6$ & sicl & $1 \mathrm{msbk}$ & firm & 6 & NA \\
\hline 128 & WAKELAND & 0 & NA & A & $0-6$ & $10 y r 4 / 3$ cff $10 y r 4 / 2$ & sil & $2 m s b k$ & $\mathrm{fr}$ & 6 & Cs \\
\hline 128 & WAKELAND & 0 & NA & Bw1 & $006-16$ & $10 y r 5 / 3$ cff $10 y r 5 / 2,4 / 2$ & sil & $2 m s b k$ & $\mathrm{fr}$ & 6 & Cs \\
\hline 128 & WAKELAND & 0 & NA & Bw2 & $16-25$ & $10 y r 5 / 2$ cff $5 / 3,5 / 8$ & sil & $2 m s b k$ & $\mathrm{fr}$ & 5.5 & Cs \\
\hline
\end{tabular}




\begin{tabular}{|c|c|c|c|c|c|c|c|c|c|c|c|}
\hline Site & Series & Slope & Aspect & Horizon & Depth & Moist Color & Texture & Structure & Consistency & $\mathbf{P h}$ & Boundary \\
\hline 128 & WAKELAND & 0 & NA & Bw3 & $25-40$ & $10 \mathrm{yr} 5 / 2 \mathrm{cff} 5 / 2,5 / 8$ & sil & $2 m s b k$ & $\mathrm{fr}$ & 6 & Cs \\
\hline 128 & WAKELAND & 0 & NA & C & $40-60$ & $10 y r 5 / 2$ cff $10 y r 4 / 2,5 / 8$ & sil & massive & $\mathrm{fr}$ & 6 & Cs \\
\hline 129 & LENZBURG & 0 & NA & $\mathrm{AP}$ & $0-5$ & $\operatorname{mix} 5 y \mathrm{r} 5 / 1 \mathrm{fl} 10 \mathrm{yr} 5 / 85 \%$ coal & cl $5 \%$ coal & $\mathrm{cp}$ & firm & $7.5, \mathrm{FC}$ & As \\
\hline 129 & LENZBURG & 0 & NA & $\mathrm{B} / \mathrm{C} 1$ & $005-16$ & $\operatorname{mix} 10 y r 4 / 2,4 / 3,5 / 6$ & sil \& sicl & $\mathrm{cp}$ & vfirm & 6 & As \\
\hline 129 & LENZBURG & 0 & NA & $\mathrm{B} / \mathrm{C} 2$ & $16-30$ & $\operatorname{mix} 10 y r 5 / 1,2.5 y r 6 / 1$ & hvy cl & $\mathrm{cp}$ & $v$ firm & 6 & As \\
\hline 129 & LENZBURG & 0 & NA & $\mathrm{B} / \mathrm{C} 3$ & $30-60$ & $\operatorname{mix} 10 y r 5 / 1 \& 5 / 6$ & hvy cl & $\mathrm{cp}$ & $v$ firm & $7.5, \mathrm{FC}$ & As \\
\hline 130 & LENZBURG & 1 & $\mathrm{~N}$ & $\mathrm{~B} / \mathrm{C} 1$ & $0-30$ & $\begin{array}{l}\text { mix } 5 y r 4 / 1410 y r 5 / 210 y r 5 / 65-10 \% \\
\text { shale fragel coal farm }\end{array}$ & hvy cl & $\mathrm{cp}$ & $v$ firm & $7.5, \mathrm{FC}$ & As \\
\hline 130 & LENZBURG & 1 & $\mathrm{~N}$ & $\mathrm{~B} / \mathrm{C} 2$ & $30-60$ & mixed $10 y r$ 4/11,5/6 & $\mathrm{cl}$ & $\mathrm{cp}$ & $v$ firm & $7.5, \mathrm{FC}$ & NA \\
\hline 131 & na & 8 & $E$ & AP & $0-8$ & $10 y r 4 / 2$ & sil & $1 \mathrm{fgr}$ & $\mathrm{fr}$ & 5.5 & As \\
\hline 131 & na & 8 & $E$ & $\mathrm{~B} / \mathrm{C} 1$ & $008-26$ & $2.5 y r 4 / 110 y r 6 / 2$ cfd $10 y r 5 / 6$ & hvy cl & $\mathrm{cp}$ & $\checkmark$ firm & $7.5, \mathrm{FC}$ & AS \\
\hline 131 & na & 8 & $E$ & $\mathrm{~B} / \mathrm{C} 2$ & $26-60$ & $\operatorname{mix} 10 y r 5 / 6,2.5$ yr $5 / 2$ & hvy cl & $\mathrm{cp}$ & $v$ firm & 7 & NA \\
\hline 132 & SCHULINE & 25 & $\mathrm{~N}$ & AP & $0-4$ & $10 y r 4 / 2$ & sil & $2 \mathrm{fgr}$ & $\mathrm{fr}$ & 6.5 & As \\
\hline 132 & SCHULINE & 25 & $\mathrm{~N}$ & $\mathrm{~B} / \mathrm{C} 1$ & $004-11$ & $\operatorname{mix} 10 y r 4 / 2,5 / 18,5 / 6$ & sil & $1 \mathrm{fsbk}$ & $\mathrm{fr}$ & 7 & As \\
\hline 132 & SCHULINE & 25 & $\mathrm{~N}$ & $\mathrm{~B} / \mathrm{C} 2$ & $011-30$ & $\operatorname{mix} 10 y r 5 / 1,10 y r 5 / 3,5 / 8$ & hvy cl & $\mathrm{cp}$ & firm & $7.5, \mathrm{FC}$ & As \\
\hline 132 & SCHULINE & 25 & $\mathrm{~N}$ & $\mathrm{~B} / \mathrm{C} 3$ & $30-60$ & $\operatorname{mix} 10 y r 5 / 85 / 65 / 8 \mathrm{cfd}$ & hvy cl & $\mathrm{cp}$ & $v$ firm & $7.5, \mathrm{FC}$ & As \\
\hline 133 & SCHULINE & 0 & NA & $A$ & $0-6$ & $\begin{array}{l}10 \mathrm{yr} 4 / 2 \text { some in mixing of subs } 5 / 6 \text {, } \\
5 \mathrm{lbs}\end{array}$ & sil & $1 \mathrm{fgr}$ & $\mathrm{fr}$ & 6 & As \\
\hline 133 & SCHULINE & 0 & NA & $\mathrm{B} / \mathrm{C} 1$ & 006-17 & $10 \mathrm{yr} 5 / 11 \mathrm{ft} 10 \mathrm{yr} 5 / 6$ & $\mathrm{cl} 5 \mathrm{~g}$ & $\mathrm{cp}$ & firm & $7.5, \mathrm{FC}$ & As \\
\hline 133 & SCHULINE & 0 & NA & $\mathrm{B} / \mathrm{C} 2$ & $17-32$ & $\operatorname{mix} 10 y r 5 / 1,2.56 / 2$ & $\mathrm{cl} \&$ sicl & $\mathrm{cp}$ & firm & $7.5, \mathrm{FC}$ & As \\
\hline
\end{tabular}




\begin{tabular}{|c|c|c|c|c|c|c|c|c|c|c|c|}
\hline Site & Series & Slope & Aspect & Horizon & Depth & Moist Color & Texture & Structure & Consistency & $\mathbf{P h}$ & Boundary \\
\hline 133 & SCHULINE & 0 & NA & $\mathrm{B} / \mathrm{C} 3$ & $32-60$ & $\operatorname{mix} 10 y r 5 / 6,5 / 8,5 / 1$ & cl $5 \%$ & c & $v$ firm & $7.5, \mathrm{FC}$ & AS \\
\hline 136 & na & 0 & NA & NA & NA & Old Road Bed Gravely Rock & NA & NA & NA & NA & NA \\
\hline 137 & HOSMER & 0 & NA & AP & $0-7$ & f.conc. $10 y r 4 / 2$ & sil & $2 \mathrm{fgr}$ & $\mathrm{fr}$ & 5.5 & As \\
\hline 137 & HOSMER & 0 & NA & Bt1 & $007-16$ & conc. $10 \mathrm{yr} 5 / 4 \mathrm{cfd} 5 / 2,4 / 6 \mathrm{c} 5 / 2$ & sicl & $2 \mathrm{fsbk}$ & $\mathrm{fr}$ & 5 & Cs \\
\hline 137 & HOSMER & 0 & NA & Btg1 & $16-30$ & conc. $10 \mathrm{yr} 5 / 2 \mathrm{cfd} 5 / 8,4 / 6$ & sicl & $2 f s b k$ & firm & 5 & Cs \\
\hline 137 & HOSMER & 0 & NA & Btg2 & $30-42$ & $5 / 1$ cfd $5 / 8,4 / 6$ c $5 / 1$ & sicl & $2 \mathrm{fsbk}$ & firm & 6.5 & Cs \\
\hline 137 & HOSMER & 0 & NA & $\operatorname{Btg} 3$ & $42-60$ & $5 / 1$ cfd $5 / 8,5 / 4,6 / 2$ c $5 / 1$ & sicl & $2 f s b k$ & firm & 6.5 & \\
\hline 138 & WAKELAND & 0 & NA & $A$ & $0-7$ & 10yr $4 / 310 y r 4 / 2 \mathrm{cmfaint}$ & sil & $1 \mathrm{msbk}$ & $\mathrm{fr}$ & 6 & Cs \\
\hline 138 & WAKELAND & 0 & NA & Bw1 & $007-16$ & $10 y r 5 / 3 \mathrm{cmf} 5 / 2,5 / 9$ & sil & $1 \mathrm{msbk}$ & $\mathrm{fr}$ & 6 & Cs \\
\hline 138 & WAKELAND & 0 & NA & Bw2 & $16-40$ & $10 \mathrm{yr} 5 / 2 \mathrm{mmf} 5 / 3 \mathrm{ffd} 5 / 8$ & sil & $1 \mathrm{msbk}$ & $\mathrm{fr}$ & 6 & Cs \\
\hline 138 & WAKELAND & 0 & NA & Bw3 & $40-60$ & $10 \mathrm{yr} 5 / 2 \mathrm{cfd} 5 / 3,5 / 8$ & sil & $1 \mathrm{msbk}$ & $\mathrm{fr}$ & 6 & Cs \\
\hline 139 & LENZBURG & 12 & $\mathrm{~N}$ & only & $0-60$ & $\operatorname{mix} 5 y r 5 / 110 y r 5 / 6$ & hvy cl & $\mathrm{cp}$ & $v$ firm & $7.5, \mathrm{FC}$ & NA \\
\hline 139 & LENZBURG & 12 & $\mathrm{~N}$ & NA & NA & $5-10 \%$ gravel coal \& shale frig. & NA & NA & NA & NA & NA \\
\hline 140 & $\begin{array}{l}\text { SCHULINE-NON } \\
\text { CALCAREOUS }\end{array}$ & 5 & NW & AP & $0-3$ & conc. $10 \mathrm{yr} 4 / 2$ & Sil & $1 \mathrm{fsbk}$ & $\mathrm{fr}$ & 6 & AS \\
\hline 140 & $\begin{array}{l}\text { SCHULINE-NON } \\
\text { CALCAREOUS }\end{array}$ & 5 & NW & $\mathrm{B} / \mathrm{C} 1$ & $003-10$ & $\operatorname{mix} 10 y r 5 / 4,5 / 2$ cf $10 y r 5 / 8$ & Sicl, sil & $\mathrm{cp} \& \mathrm{sbk}$ & $\mathrm{fr}$ & 6.5 & AS \\
\hline 140 & $\begin{array}{l}\text { SCHULINE-NON } \\
\text { CALCAREOUS }\end{array}$ & 5 & NW & $\mathrm{B} / \mathrm{C} 2$ & 010-24 & $\operatorname{mix} 10 y r 5 / 1,5 / 4$ cf $10 y r 5 / 8$ & Sicl & $c p \& s b k$ & $\mathrm{fr}$ & 6.5 & AS \\
\hline 140 & $\begin{array}{l}\text { SCHULINE-NON } \\
\text { CALCAREOUS }\end{array}$ & 5 & NW & $\mathrm{B} / \mathrm{C} 3$ & $24-60$ & $\operatorname{mix} 10 y r 6 / 2,5 / 6,4 / 1$ & cl. Till 5-10\% & $\mathrm{CP}$ & $\checkmark$ firm & 7 & NA \\
\hline
\end{tabular}




\begin{tabular}{|c|c|c|c|c|c|c|c|c|c|c|c|}
\hline Site & Series & Slope & Aspect & Horizon & Depth & Moist Color & Texture & Structure & Consistency & $\mathbf{P h}$ & Boundary \\
\hline 141 & $\begin{array}{l}\text { SCHULINE-NON } \\
\text { CALCAREOUS }\end{array}$ & 6 & NW & AP & $0-3$ & conc. $10 y r 4 / 2$ & sil & $1 \mathrm{fsbk}$ & $\mathrm{fr}$ & 6 & AS \\
\hline 141 & $\begin{array}{l}\text { SCHULINE-NON } \\
\text { CALCAREOUS }\end{array}$ & 6 & NW & $\mathrm{B} / \mathrm{C} 1$ & 003-10 & $\operatorname{mix} 10 y r 5 / 4,5 / 2$ cf $10 y r 5 / 8$ & sicl, sil & $\mathrm{cp} \& \mathrm{sbk}$ & $\mathrm{fr}$ & 6.5 & AS \\
\hline 141 & $\begin{array}{l}\text { SCHULINE-NON } \\
\text { CALCAREOUS }\end{array}$ & 6 & NW & $\mathrm{B} / \mathrm{C} 2$ & $010-24$ & $\operatorname{mix} 10 y r 5 / 1,5 / 4 /$ cf $10 y r 5 / 8$ & sil, sicl & $\mathrm{cp}, \mathrm{sbk}$ & $\mathrm{fr}$ & 6.5 & AS \\
\hline 141 & $\begin{array}{l}\text { SCHULINE-NON } \\
\text { CALCAREOUS }\end{array}$ & 6 & NW & $\mathrm{B} / \mathrm{C} 3$ & $24-60$ & $\operatorname{mix} 10 y r 6 / 2,5 / 6,4 / 1$ & cl.Till 5-10\% & $\mathrm{CP}$ & $v$ firm & 7 & NA \\
\hline 142 & SCHULINE & 1 & $E$ & A & $0-4$ & $m \times 10 y r 5 / 1,4 / 2$ cfd $5 / 8$ & sil & $1 \mathrm{fsbk}$ & $\mathrm{fr}$ & 6 & As \\
\hline 142 & SCHULINE & 1 & $E$ & $\mathrm{~B} / \mathrm{C} 1$ & $004-18$ & $\mathrm{mx} 10 \mathrm{yr} 5 / 1,5 / 6 \mathrm{cfd} 5 / 8$ & cl.till 5\% & $\mathrm{cp}$ & $v$ firm & $7.5, \mathrm{FC}$ & As \\
\hline 142 & SCHULINE & 1 & $E$ & $\mathrm{~B} / \mathrm{C} 2$ & $18-30$ & $m x 10 y r 5 / 6,5 / 2,5 / 1, \mathrm{~cd} 5 / 8$ & hvy cl 5\% & $\mathrm{cp}$ & $v$ firm & $7.5, \mathrm{FC}$ & As \\
\hline 142 & SCHULINE & 1 & $E$ & $\mathrm{~B} / \mathrm{C} 3$ & $30-60$ & $m \times 10 y r 5 / 1,6 / 8,5 / 6$ & cl $5 \%$ coal & $\mathrm{cp}$ & $v$ firm & $7.5, \mathrm{FC}$ & As \\
\hline 143 & LENZBURG & 3 & NNW & AP & $0-7$ & mixed $10 y r 5 / 2 \& 5 / 6$ com $5 / 8$ & SIL & $1 \mathrm{fsbk}$ & $\mathrm{fr}$ & 5.5 & As \\
\hline 143 & LENZBURG & 3 & NNW & CB1 & $007-17$ & mixed $10 y r 5 / 1 \& 2.5 \& 6 / 2$ & cl10\% coal & $\mathrm{CP}$ & vfirm & 6.5 & As \\
\hline 143 & LENZBURG & 3 & NNW & $\mathrm{B} / \mathrm{C} 2$ & $17-26$ & mixed $10 y r 5 / 4,5 / 2 \& 5 / 8$ & cl5-10\% co & CP \& Tills & vfirm & 7 & As \\
\hline 143 & LENZBURG & 3 & NNW & C/B2 & $26-60$ & mixed $10 y r 5 / 2,6 / 1 \& 5 / 6$ & cl5-10\%co & $\mathrm{CP}$ & vfirm & $9.5, \mathrm{FC}$ & NA \\
\hline 144 & SCHULINE & 6 & $E$ & AP & $0-4$ & Mixed10yr 4/3 \& 4/2 & SIL & 1 far & $\mathrm{fr}$ & 6 & As \\
\hline 144 & SCHULINE & 6 & $E$ & $\mathrm{~B} / \mathrm{C} 1$ & $004-12$ & mixed10yr 5/2, 4/2, 4/3 & SIL & $\mathrm{cp}$ & $\mathrm{fr}$ & 5.5 & As \\
\hline 144 & SCHULINE & 6 & $E$ & $\mathrm{~B} / \mathrm{C} 2$ & $012-27$ & mixed10yr $4 / 3,6 / 2,5 / 1$ cf $5 / 8$ & sil,sicl, cl & $\mathrm{cp}$ & vfirm & $7.5, \mathrm{FC}$ & As \\
\hline 144 & SCHULINE & 6 & $E$ & $\mathrm{~B} / \mathrm{C} 3$ & $27-60$ & mixed10yr $4 / 3,5 / 1$ cf $10 y r 5 / 8$ & hvycl5-10\% & $\mathrm{CP}$ & vfirm & $7.5, \mathrm{FC}$ & NA \\
\hline 146 & LENZBURG & 3 & W & $A$ & $0-5$ & mixed $10 y r 5 / 4,5 / 8,5 / 2$ & cl $5 \%$ & $1 \mathrm{msbk}$ & firm & 6 & As \\
\hline
\end{tabular}




\begin{tabular}{|c|c|c|c|c|c|c|c|c|c|c|c|}
\hline Site & Series & Slope & Aspect & Horizon & Depth & Moist Color & Texture & Structure & Consistency & $\mathbf{P h}$ & Boundary \\
\hline 146 & LENZBURG & 3 & W & $\mathrm{B} / \mathrm{C} 1$ & $005-10$ & mixed $10 y r$ 4/1, $10 y r 5 / 4$ & cl \& sicl 5\% & mass $\mathrm{cp}$ & vfirm & $7.5, \mathrm{FC}$ & As \\
\hline 146 & LENZBURG & 3 & W & $\mathrm{B} / \mathrm{C} 2$ & 010-22 & mixed $10 y r 4 / 1,10 y r 5 / 4$ & hvy cl & mass $\mathrm{cp}$ & vfirm & $7.5, \mathrm{FC}$ & As \\
\hline 146 & LENZBURG & 3 & W & $\mathrm{B} / \mathrm{C} 3$ & $22-60$ & mixed 5yr 5/1, 10yr 4/1 & clay shale & mass $\mathrm{cp}$ & vfirm & $7.5, \mathrm{FC}$ & NA \\
\hline 148 & SCHULINE & 4 & $\mathrm{~N}$ & $A$ & $0-4$ & mixed $10 y r$ 4/1, 5/2, 4/3 & SIL & $1 \mathrm{fsbk}$ & fr & 6 & As \\
\hline 148 & SCHULINE & 4 & $\mathrm{~N}$ & $\mathrm{~B} / \mathrm{C} 1$ & $004-16$ & mixed $10 y r 5 / 1,4 / 2,4 / 3$ & SIL & $\mathrm{cp}$ & $\mathrm{fr}$ & $7.5, \mathrm{FC}$ & As \\
\hline 148 & SCHULINE & 4 & $\mathrm{~N}$ & $\mathrm{~B} / \mathrm{C} 2$ & $16-38$ & mixed 2.5 yr $6 / 2$ 10yr $5 / 2$ cfd10yr5/8 & SIL (E) & $\mathrm{cp}$, block & $\mathrm{fr}$ & $7.5, \mathrm{FC}$ & As \\
\hline 148 & SCHULINE & 4 & $\mathrm{~N}$ & $\mathrm{~B} / \mathrm{C} 3$ & $38-60$ & mixed $10 y r 5 / 1,4 / 2,4 / 3$ & SIL & $\mathrm{cp}$, block & $\mathrm{fr}$ & $7.5, \mathrm{FC}$ & NA \\
\hline 149 & LENZBURG & 0 & NA & AP & $0-2$ & mixed $10 y r 5 / 1,4 / 3$ & SIL & $1 \mathrm{fsbk}$ & fr & 5.5 & AS \\
\hline 149 & LENZBURG & 0 & NA & $\mathrm{B} / \mathrm{C} 1$ & $002-7$ & $10 y r 5 / 2$ cfd $10 y r 5 / 1,5 / 8$ & SIL & $\mathrm{cp}$ & fr & 6.5 & AS \\
\hline 149 & LENZBURG & 0 & NA & $\mathrm{B} / \mathrm{C} 2$ & $007-22$ & mixed $10 y r 5 / 2,5 / 6$ cfd $5 / 8$ & sil \& sicl & $\mathrm{cp}$ & fr & 7 & AS \\
\hline 149 & LENZBURG & 0 & NA & $\mathrm{B} / \mathrm{C} 3$ & $22-30$ & mixed $10 y r 4 / 3,5 / 6,5 / 1$ & cl till & $\mathrm{cp}$ & vfirm & $7.5, \mathrm{FC}$ & NA \\
\hline 150 & RIPRAP & NA & NA & NA & NA & NA & NA & NA & NA & NA & NA \\
\hline 151 & LENZBURG,NC & 8 & NE & AP & $0-8$ & mixed $10 y r 4 / 2 \& 5 / 6$ & sil \& sicl & $1 \mathrm{fsbk}$ & fr & 7 & As \\
\hline 151 & LENZBURG,NC & 8 & NE & $\mathrm{B} / \mathrm{C} 1$ & $008-28$ & mixed $10 y r$ 45/6, 4/2, 5/1 & sicl, sil & $\mathrm{cp}$ & fr & 6.5 & As \\
\hline 151 & LENZBURG,NC & 8 & NE & $\mathrm{B} / \mathrm{C} 2$ & $28-37$ & mixed $10 y r 5 / 4,5 / 6,5 / 2$ & sil & $\mathrm{cp}$ & firm & 7 & As \\
\hline 151 & LENZBURG,NC & 8 & NE & $\mathrm{B} / \mathrm{C} 3$ & $37-60$ & mixed $10 y r$ 4/1, 5/1, 5/6 & cl $5 \%$ coal & $\mathrm{Cp}$ & vfirm & 7 & NA \\
\hline 152 & SCHULINE & 1 & NE & AP & $0-7$ & mixed $10 y r$ 4/3, 4/2, ffd $10 y r 5 / 6$ & SIL & $1 \mathrm{fsbk}$ & $\mathrm{fr}$ & 6 & AS \\
\hline 152 & SCHULINE & 1 & NE & $\mathrm{B} / \mathrm{C} 1$ & $007-15$ & mixed $10 y r$ 5/1,10yr5/6 fff10yr5/8 & SIL \& SICL & $\mathrm{CP}$ & fr & 7 & AS \\
\hline
\end{tabular}




\begin{tabular}{|c|c|c|c|c|c|c|c|c|c|c|c|}
\hline Site & Series & Slope & Aspect & Horizon & Depth & Moist Color & Texture & Structure & Consistency & $\mathbf{P h}$ & Boundary \\
\hline 152 & SCHULINE & 1 & $\mathrm{NE}$ & $\mathrm{B} / \mathrm{C} 2$ & $15-60$ & mixed $5 y r 5 / 1,10 y r 4 / 6,5 / 8$ & CL10\%coal & $\mathrm{CP}$ & vfirm & $7.5, \mathrm{FC}$ & NA \\
\hline 153 & LENZBURG & 0 & NA & AP & $0-13$ & mixed $10 y r 4 / 2,5 / 1$ cfd $10 y r 5 / 1$ & sil & $1 \mathrm{fsbk}$ & $f$ & 6 & AS \\
\hline 153 & LENZBURG & 0 & NA & $\mathrm{B} / \mathrm{C} 1$ & $13-34$ & mixed10yr5/2, 4/2, 5/6cfd10yr5/8 & sil \& sicl & $\mathrm{cp}$ & $\mathrm{fr}$ & 6 & AS \\
\hline 153 & LENZBURG & 0 & NA & $\mathrm{B} / \mathrm{C} 2$ & $34-40$ & mixed10yr5/1, 5/6, 5/8 & cl $5 \% \mathrm{gr}$ & $\mathrm{cp}$ & vfirm & $7.5, \mathrm{FC}$ & AS \\
\hline 153 & LENZBURG & 0 & NA & $\mathrm{B} / \mathrm{C} 3$ & $40-60$ & $5 y r 5 / 1$ & shale resid & & vfirm & $7.5, \mathrm{FC}$ & NA \\
\hline 154 & LENZBURG & 30 & $\mathrm{~N}$ & AP & $0-12$ & mixed10yr4/2, 5/4 CFD 5/8 & sil \& sicl & $1 \mathrm{fgr}$ & $\mathrm{fr}$ & 6.5 & As \\
\hline 154 & LENZBURG & 30 & $\mathrm{~N}$ & $\mathrm{C} 1$ & $012-35$ & mixed10yr5/1, 4/2,2.5y 6/2,CFD 5/8 & sil & $\mathrm{cp}$ & $\mathrm{fr}$ & 6 & As \\
\hline 154 & LENZBURG & 30 & $\mathrm{~N}$ & $\mathrm{C} 2$ & $35-45$ & mixed10yr $4 / 1,4 / 3 ;$ CFD 5/8,4/3 & cl till & $\mathrm{cp}$ & vfirm & $7.5, \mathrm{FC}$ & As \\
\hline 154 & LENZBURG & 30 & $\mathrm{~N}$ & C3 & $45-60$ & mixed $10 y r 5 / 6,5 / 1 \mathrm{cmd} 5 / 8,4 / 3$ & cl till shale & $\mathrm{cp}$ & vfirm & $7.5, \mathrm{FC}$ & NA \\
\hline 155 & LENZBURG & 25 & W & $\mathrm{B} / \mathrm{C} 1$ & $0-10$ & $10 y r 5 / 4$ & CL 5\% & $\mathrm{cp}$ & vfirm & $7.5, \mathrm{FC}$ & As \\
\hline 155 & LENZBURG & 25 & W & $\mathrm{B} / \mathrm{C} 2$ & $010-30$ & mixed $10 y r 5 / 4,5 / 2,5 / 8$ & CL 5\% & $\mathrm{cp}$ & vfirm & $7.5, \mathrm{FC}$ & Cs \\
\hline 155 & LENZBURG & 25 & W & $\mathrm{C} / \mathrm{B}$ & $30-60$ & mixed $10 y r 5 / 6,5 / 2,5 / 8$ & cl $5 \%$ coal & $\mathrm{cp}$ & vfirm & 7 & NA \\
\hline 156 & LENZBURG & 10 & W & A & $0-7$ & mixed $10 y r 4 / 2,5 / 6$ few $6 / 2$ & sil \& sicl & $2 \mathrm{msbk}$ & $\mathrm{fr}$ & $7.5, \mathrm{FC}$ & As \\
\hline 156 & LENZBURG & 10 & W & $\mathrm{C} 1$ & $007-18$ & $5 y r 3 / 1$ cfd $10 y r 5 / 8$ & hvy cl $5 \%$ & $\mathrm{CP}$ & vfirm & $7.5, \mathrm{FC}$ & As \\
\hline 156 & LENZBURG & 10 & W & $\mathrm{C} 2$ & $18-60$ & mixed 5 yr 3/1, 10yr 5/6 & $\mathrm{cl} 5 \%$ & $\mathrm{cp}$ & vfirm & 7.5 & NA \\
\hline 157 & HAUL ROAD & NA & NA & NA & NA & COARSE GRAVEL & NA & NA & NA & NA & NA \\
\hline 158 & HAUL ROAD & NA & NA & NA & NA & COARSE GRAVEL & NA & NA & NA & NA & NA \\
\hline 159 & LENZBURG,NC & 4 & $\mathrm{~N}$ & AP & $0-5$ & mixed $10 y r$ 4/2, 5/1, 4/6 & cl $10 \%$ gr. & $1 \mathrm{fsbk}$ & $\mathrm{fr}$ & 6.5 & As \\
\hline
\end{tabular}




\begin{tabular}{|c|c|c|c|c|c|c|c|c|c|c|c|}
\hline Site & Series & Slope & Aspect & Horizon & Depth & Moist Color & Texture & Structure & Consistency & $\mathbf{P h}$ & Boundary \\
\hline 159 & LENZBURG,NC & 4 & $\mathrm{~N}$ & $\mathrm{C} 1$ & $005-11$ & mixed $5 y r 5 / 1,10 y r 5 / 6,5 / 2$ & $\mathrm{cl} 5 \% \mathrm{gr}$ & $1 \mathrm{fsbk}$ & $\mathrm{fr}$ & 5.5 & As \\
\hline 159 & LENZBURG,NC & 4 & $\mathrm{~N}$ & $\mathrm{C} 2$ & $011-27$ & mixed $5 y r 6 / 2,10 y r 5 / 2,5 / 8$ & cl shale res & massive & firm & 4.5 & As \\
\hline 159 & LENZBURG,NC & 4 & $\mathrm{~N}$ & C3 & $27-60$ & mixed $2.5 y r 4 / 1,10 y r 5 / 4,5 / 8$ & $10 \%$ shale & massive & vfirm & 5 & As \\
\hline 160 & SCHULINE & 40 & $\mathrm{~N}$ & AP & $0-3$ & $10 y r 5 / 2$ & SIL & $1 \mathrm{fsbk}$ & $\mathrm{fr}$ & 6 & As \\
\hline 160 & SCHULINE & 40 & $\mathrm{~N}$ & $\mathrm{C} 2$ & 003-13 & mixed $10 y r 5 / 6,5 / 2,5 / 1$ & SICL & $2 m s b k, c p$ & firm & 5.5 & As \\
\hline 160 & SCHULINE & 40 & $\mathrm{~N}$ & $\mathrm{C} 2$ & $13-23$ & mixed $10 y r 4 / 2,5 / 6,5 / 1$ & sil, cl 5\% & $\mathrm{cp}$ & vfirm & 5.5 & As \\
\hline 160 & SCHULINE & 40 & $\mathrm{~N}$ & $\mathrm{C} 3$ & $23-41$ & mixed $10 y r$ 4/1, 5/6 & cl $5 \%$ grale & $\mathrm{cp}$ & vfirm & 6.5 & As \\
\hline 160 & SCHULINE & 40 & $\mathrm{~N}$ & $\mathrm{C} 4$ & $41-60$ & mixed 5yr 4/1, 10yr 5/2 ;CFD 5/8 & cl till & $\mathrm{cp}$ & vfirm & $7.5, \mathrm{FC}$ & NA \\
\hline 161 & SCHULINE & 1 & NA & $A$ & $0-5$ & $10 y r 4 / 3$ & sil & $1 \mathrm{fgr}$ & $\mathrm{fr}$ & 5.5 & As \\
\hline 161 & SCHULINE & 1 & NA & $\mathrm{BA}$ & $005-24$ & $10 y r 4 / 3,10 y r 5 / 2,10 y r 4 / 6$ & sicl & 1tkpl & $\mathrm{fi}$ & 6 & As \\
\hline 161 & SCHULINE & 1 & NA & C & $24-60$ & $10 y r 4 / 6,5 y r 5 / 1, N 2 / 0$ & $\mathrm{cl}$ & M & efi & $8, \mathrm{FC}$ & NA \\
\hline 162 & LENZBURG & 7 & NA & $\mathrm{BC}$ & $0-5$ & $10 y r 5 / 6$ & sicl & $\mathrm{cp}$ & vfi & 6 & As \\
\hline 162 & LENZBURG & 7 & NA & $\mathrm{C}$ & $005-60$ & $5 \mathrm{Yr} 6 / 1, \mathrm{~N} 2 / 0,2.5 \mathrm{Yr} 4 / 2$ & $\mathrm{cl}$ & $\mathrm{m}$ & efi & $8, \mathrm{FC}$ & NA \\
\hline 163 & SCHULINE & 36 & NA & $A$ & $0-4$ & $10 y r 4 / 3$ & sil & $1 \mathrm{fgr}$ & $\mathrm{fr}$ & 6 & AS \\
\hline 163 & SCHULINE & 36 & NA & $\mathrm{BC}$ & $004-20$ & $10 \mathrm{yr} 4 / 3,10 \mathrm{yr} 4 / 6$ & sil & $1 \mathrm{fsbk}$ & $\mathrm{fi}$ & 5.5 & AS \\
\hline 163 & SCHULINE & 36 & NA & C & $20-60$ & 10 yr 4/6, N 2/0, 5yr 5/1 & $\mathrm{cl}$ & $\mathrm{m}$ & efi & $8, \mathrm{FC}$ & NA \\
\hline 164 & SCHULINE & 4 & NA & $A$ & $0-5$ & $10 y r 4 / 3$ & sil & $1 \mathrm{fgr}$ & $\mathrm{fr}$ & 5.5 & As \\
\hline 164 & SCHULINE & 4 & NA & $\mathrm{A} / \mathrm{B}$ & 005-20 & $10 y r 5 / 2,10 y r$ 4/3, 10 yr 4/6 mix & sil & $1 \mathrm{fsbk}$ & $\mathrm{fi}$ & 5.5 & As \\
\hline
\end{tabular}




\begin{tabular}{|c|c|c|c|c|c|c|c|c|c|c|c|}
\hline Site & Series & Slope & Aspect & Horizon & Depth & Moist Color & Texture & Structure & Consistency & $\mathbf{P h}$ & Boundary \\
\hline 164 & SCHULINE & 4 & NA & $\mathrm{B} / \mathrm{C}$ & $20-40$ & 2.5 yr 5/1, 10yr 4/6 & sicl & $\mathrm{cp}$ & fi & $7.5, \mathrm{FC}$ & Cs \\
\hline 164 & SCHULINE & 4 & NA & $\mathrm{C}$ & $40-60$ & 5YR 6/1 (Gray shale) & 1 & $\mathrm{~m}$ & efi & $8, \mathrm{FC}$ & NA \\
\hline 165 & LENZBURG,NC & 3 & SE & AP & $0-4$ & $\operatorname{mix} 10 y r 4 / 2,5 / 6$ & sil, cl & $1 \mathrm{fsbk}$ & firm & 6 & As \\
\hline 165 & LENZBURG,NC & 3 & SE & $\mathrm{B} / \mathrm{C} 1$ & $004-17$ & $\operatorname{mix} 10 y r 5 / 2,5 / 6$ wc $5 / 8,5 / 1$ & Ioam & $\mathrm{cp}$ & firm & 5.5 & Cs \\
\hline 165 & LENZBURG,NC & 3 & SE & $\mathrm{B} / \mathrm{C} 2$ & $17-30$ & $\operatorname{mix} 10 y r 5 / 6,5 / 2 \quad C 5 / 8$ & $\mathrm{cl}$ & $\mathrm{cp}$ & firm & 5.5 & As \\
\hline 165 & LENZBURG,NC & 3 & SE & $\mathrm{B} / \mathrm{C} 3$ & $30-60$ & $\operatorname{mix} 10 y r 5 / 2,5 / 6,4 / 6$ & cl \& sil & $\mathrm{cp}$ & firm & 6 & NA \\
\hline 166 & BIRDS & 0 & NA & $A$ & $0-4$ & $10 \mathrm{YR} 4 / 2$ & sil & $2 \mathrm{fgr}$ & $\mathrm{fr}$ & 6 & As \\
\hline 166 & BIRDS & 0 & NA & Bwg1 & $004-11$ & $10 \mathrm{yr} 4 / 2$, cf $10 \mathrm{yr} 5 / 8$ & sil & $1 \mathrm{fsbk}$ & $\mathrm{fr}$ & 6.5 & Cs \\
\hline 166 & BIRDS & 0 & NA & Bwg2 & $011-20$ & $10 y r 5 / 2$ cf $10 y r 5 / 8$ & sil & $1 \mathrm{fsbk}$ & $\mathrm{fr}$ & 6.5 & Cs \\
\hline 166 & BIRDS & 0 & NA & $\mathrm{Cg} 1$ & $20-40$ & $2.5 \mathrm{yr} 6 / 2 \mathrm{cf} \& \mathrm{~m} 5 / 8,4 / 010 \mathrm{yr}$ & sil & massive & $\mathrm{fr}$ & 6.5 & Cs \\
\hline 166 & BIRDS & 0 & NA & $\mathrm{Cg} 2$ & $40-60$ & $2.5 \mathrm{yr} 6 / 2 \mathrm{~m} \mathrm{10yr} 5 / 8,4 / 6$ & sil & massive & $\mathrm{fr}$ & 6.5 & Cs \\
\hline 167 & BIRDS & 0 & NA & $A$ & $0-3$ & $10 y r 4 / 2$ & sil & $2 \mathrm{fgr}$ & fr & 6.5 & AS \\
\hline 167 & BIRDS & 0 & NA & Bwg1 & $003-8$ & $10 y r 5 / 2$ ff $10 y r 5 / 8$ & sil & $1 \mathrm{fsbk}$ & $\mathrm{fr}$ & 6.5 & CS \\
\hline 167 & BIRDS & 0 & NA & Bwg2 & $008-16$ & $10 y r 5 / 2$ cfd $10 y r 5 / 8$ & sil & $1 \mathrm{fsbk}$ & $\mathrm{fr}$ & 6.5 & CS \\
\hline 167 & BIRDS & 0 & NA & Bwg3 & $16-30$ & $2.5 \mathrm{yr} 6 / 2 \mathrm{cfd} 10 \mathrm{yr} 4 / 6,5 / 8$ & sil & $1 \mathrm{fsbk}$ & $\mathrm{fr}$ & 6.5 & CS \\
\hline 167 & BIRDS & 0 & NA & $\mathrm{Cg}$ & $30-60$ & $2.5 \mathrm{yr} 6 / 2 \mathrm{~m} \mathrm{10yr} 4 / 6,5 / 8$ & sil & massive & $\mathrm{fr}$ & 6.5 & NA \\
\hline 168 & BIRDS & 0 & NA & $A$ & $0-6$ & $10 y r 4 / 2$ fom $5 / 2 \& 10 y r 5 / 5$ & sil & $2 \mathrm{fgr}$ & $\mathrm{fr}$ & 6.2 & Cs \\
\hline 168 & BIRDS & 0 & NA & Bw1 & $006-20$ & $10 y r 6 / 1$ cfd $10 y r$ r $4 / 6,5 / 8$ & sil & $1 \mathrm{msbk}$ & $\mathrm{fr}$ & 6 & Cs \\
\hline
\end{tabular}




\begin{tabular}{|c|c|c|c|c|c|c|c|c|c|c|c|}
\hline Site & Series & Slope & Aspect & Horizon & Depth & Moist Color & Texture & Structure & Consistency & $\mathbf{P h}$ & Boundary \\
\hline 168 & BIRDS & 0 & NA & Bw2 & $20-36$ & $2.5 \mathrm{yr} 6 / 2 \mathrm{~m} 10 \mathrm{yr} 5 / 8,5 / 2$ & sil & $1 \mathrm{msbk}$ & $\mathrm{fr}$ & 6 & Cs \\
\hline 168 & BIRDS & 0 & NA & C & $36-80$ & $2.5 \mathrm{yr} 6 / 2 \mathrm{md} 5 / 8,4 / 6$ & sil & massive & $\mathrm{fr}$ & 6.5 & Cs \\
\hline 169 & HAUL ROAD & NA & NA & NA & NA & NA & NA & NA & NA & NA & NA \\
\hline 170 & SCHULINE & 0 & NA & $A$ & $0-5$ & $10 \mathrm{yr} 4 / 3$ & sil & $1 \mathrm{fgl}$ & $\mathrm{fr}$ & 5.5 & As \\
\hline 170 & SCHULINE & 0 & NA & $\mathrm{B} / \mathrm{C} 1$ & $005-20$ & $10 \mathrm{yr} 4 / 2,2.5 \mathrm{yr} 5 / 110 \mathrm{yr} 4 / 6$ & sicl & $\mathrm{cp}$ & vfi & $7.5, \mathrm{FC}$ & Cs \\
\hline 170 & SCHULINE & 0 & NA & $\mathrm{B} / \mathrm{C} 2$ & $20-38$ & $2.5 \mathrm{yr} 5 / 1,10 \mathrm{yr} 4 / 6$ & sicl & $\mathrm{cp}$ & vfi & $8, F C$ & Cs \\
\hline 170 & SCHULINE & 0 & NA & C & $38-60$ & 5 yr $5 / 1$ shale & $\mathrm{cl}$ & $\mathrm{m}$ & efi & $8, \mathrm{FC}$ & NA \\
\hline 171 & WAKELAND & 0 & NA & $A$ & $0-5$ & $10 \mathrm{yr} 4 / 5$ & sil & $1 \mathrm{fgr}$ & $\mathrm{fr}$ & 6 & As \\
\hline 171 & WAKELAND & 0 & NA & $\mathrm{Bg} 1$ & 005-12 & $10 y r 5 / 6$ cp $10 y r 5 / 2$ & sil & $1 \mathrm{fsbk}$ & $\mathrm{fr}$ & 5.8 & Cs \\
\hline 171 & WAKELAND & 0 & NA & $\mathrm{Bg} 2$ & $012-20$ & 10yr 4/6 CP 10yr 5/1 & sil & $1 \mathrm{fsbk}$ & $\mathrm{fr}$ & 5.8 & Cs \\
\hline 171 & WAKELAND & 0 & NA & Bw1 & $20-28$ & 10 yr $5 / 2$ cr cd $10 y r 4 / 3$ & sil & $1 \mathrm{msbk}$ & $\mathrm{fr}$ & 6 & Cs \\
\hline 171 & WAKELAND & 0 & NA & Bw2 & $28-42$ & $10 y r 5 / 2$ cf $10 y r 5 / 3$ & sil & $1 \mathrm{msbk}$ & $\mathrm{fr}$ & 6.2 & Cs \\
\hline 171 & WAKELAND & 0 & NA & $\mathrm{Bg} 1$ & $42-60$ & $10 y r$ r $5 / 6$ cp $10 y r$ 5/1 & sil & $1 \mathrm{msbk}$ & $\mathrm{fr}$ & 6.2 & NA \\
\hline 172 & SCHULINE & 1 & NA & $A$ & $0-5$ & $10 y r 4 / 3$ & sil & $1 \mathrm{fgr}$ & $\mathrm{fr}$ & 6 & As \\
\hline 172 & SCHULINE & 1 & NA & $\mathrm{B} / \mathrm{C} 1$ & 005-15 & $10 y r 4 / 3,10 y r 4 / 6$ & sicl & $\mathrm{cp}$ & $\mathrm{fi}$ & 6.5 & Cs \\
\hline 172 & SCHULINE & 1 & NA & $\mathrm{B} / \mathrm{C} 2$ & $15-44$ & $10 y r 5 / 6,10 y r 4 / 2,2.5$ yr $5 / 1$ & sicl & $\mathrm{cp}$ & vfi & $8, \mathrm{FC}$ & Cs \\
\hline 172 & SCHULINE & 1 & NA & $\mathrm{B} / \mathrm{C} 3$ & $44-60$ & 2.5 yr $5 / 1, N 2 / 0$ & $\mathrm{~L}$ & M & efi & $8, \mathrm{FC}$ & NA \\
\hline 173 & SCHULINE & 1 & NA & A & $0-8$ & $10 y r 4 / 3$ & sil & $1 \mathrm{mgr}$ & $\mathrm{fr}$ & 6.8 & As \\
\hline
\end{tabular}




\begin{tabular}{|c|c|c|c|c|c|c|c|c|c|c|c|}
\hline Site & Series & Slope & Aspect & Horizon & Depth & Moist Color & Texture & Structure & Consistency & $\mathbf{P h}$ & Boundary \\
\hline 173 & SCHULINE & 1 & NA & $\mathrm{B} / \mathrm{C} 1$ & $008-27$ & $10 y r 5 / 4,10 y r 5 / 6$ & sicl & fvcp & $\mathrm{fi}$ & $8, \mathrm{FC}$ & Cs \\
\hline 173 & SCHULINE & 1 & NA & $\mathrm{B} / \mathrm{C} 2$ & $27-43$ & 2.5 yr 5/1 10yr 5/8 & sicl & fvcp & vfi & $8, \mathrm{FC}$ & Cs \\
\hline 173 & SCHULINE & 1 & NA & $\mathrm{B} / \mathrm{C} 3$ & $43-60$ & $10 y r 5 / 8,10 y r 5 / 1$ & $\mathrm{cl}$ & $\mathrm{m}$ & efi & $8, \mathrm{FC}$ & NA \\
\hline 174 & LENZBURG & 1 & NA & A & & $10 \mathrm{yr} 4 / 4$ & sil & $1 \mathrm{fgr}$ & $\mathrm{fr}$ & 6 & As \\
\hline 174 & LENZBURG & 1 & NA & C1 & $004-20$ & $10 y r 4 / 65$ yr 5/1, N 2/0 coal mix & $\mathrm{cl}$ & $\mathrm{m}$ & efi & 5 & Cs \\
\hline 174 & LENZBURG & 1 & NA & $\mathrm{C} 2$ & $20-60$ & $\mathrm{~N} 2 / 0,10 y r 4 / 6,5$ yr $5 / 1$ & $\mathrm{cl}$ & $\mathrm{m}$ & efi & $8, \mathrm{FC}$ & NA \\
\hline 175 & SCHULINE & 4 & NA & A & $0-5$ & $10 \mathrm{yr} 4 / 3$ & sil & $1 \mathrm{fgr}$ & $\mathrm{fr}$ & 5.5 & As \\
\hline 175 & SCHULINE & 4 & NA & $\mathrm{B} / \mathrm{C} 1$ & $005-11$ & $10 y r 4 / 310 y r 4 / 6$ & sil & $1 \mathrm{fsbk}$ & $\mathrm{fi}$ & 6 & As \\
\hline 175 & SCHULINE & 4 & NA & $\mathrm{B} / \mathrm{C} 2$ & $011-45$ & $7.5 \mathrm{yr} 4 / 6,5 \mathrm{yr} 2 / 1,10 \mathrm{yr} 4 / 4$ & sicl & $\mathrm{cp}$ & vfi & $8, \mathrm{FC}$ & Cs \\
\hline 175 & SCHULINE & 4 & NA & $\mathrm{B} / \mathrm{C} 3$ & $45-60$ & $7.5 \mathrm{yr} 4 / 8, \mathrm{~N} 2 / 0$ 10yr $4 / 4$ & $\mathrm{cl}$ & $\mathrm{m}$ & efi & $8, \mathrm{FC}$ & NA \\
\hline 176 & SCHULINE & 2 & NA & A & $0-5$ & $10 y r 4 / 3$ & sil & $1 \mathrm{fgr}$ & $\mathrm{fr}$ & 6 & As \\
\hline 176 & SCHULINE & 2 & NA & $A B$ & $005-12$ & $10 y r 4 / 6,10 y r$ 4/3 & sil & $1 \mathrm{fsbk}$ & fr & 6 & As \\
\hline 176 & SCHULINE & 2 & NA & C1 & $012-30$ & $\mathrm{~N} 2 / 0,10 y r 4 / 2,10 y r$ 4/6 mix coal & sicl & $\mathrm{m}$ & efi & $8, \mathrm{FC}$ & Cs \\
\hline 176 & SCHULINE & 2 & NA & $\mathrm{C} 2$ & $30-60$ & $\mathrm{~N} 2 / 0,10 \mathrm{yr} 4 / 2,10 \mathrm{yr} 4 / 6,2.5 \mathrm{y} 5 / 1$ & $\mathrm{cl}$ & $\mathrm{m}$ & efi & $8, \mathrm{FC}$ & NA \\
\hline 177 & LENZBURG & 19 & NA & A & $0-5$ & $10 y r 4 / 3$ & sil & $1 \mathrm{fgr}$ & $\mathrm{fr}$ & 6 & As \\
\hline 177 & LENZBURG & 19 & NA & $A / B$ & $005-13$ & $10 y r$ 4/3, 10yr 4/6 & sil & 1 fsbk & $\mathrm{fi}$ & 6 & As \\
\hline 177 & LENZBURG & 19 & NA & C & $13-20$ & gray shale 5 yr $6 / 1$ & shale silty & $2 \mathrm{tkpl}$ & efi & $7.5, \mathrm{FC}$ & NA \\
\hline 178 & SCHULINE,NC & 0 & NA & AP & $0-7$ & mixed 10yr 5/4, 5/6 & sil \& cl & $1 \mathrm{fsbk}$ & $\mathrm{fr}$ & 6.5 & As \\
\hline
\end{tabular}




\begin{tabular}{|c|c|c|c|c|c|c|c|c|c|c|c|}
\hline Site & Series & Slope & Aspect & Horizon & Depth & Moist Color & Texture & Structure & Consistency & $\mathbf{P h}$ & Boundary \\
\hline 178 & SCHULINE,NC & 0 & NA & $\mathrm{B} / \mathrm{C} 1$ & $007-16$ & mixed $10 y r 5 / 4,5 / 2$ & $\mathrm{cl}$ & $\mathrm{cp}$ & firm & 6.5 & As \\
\hline 178 & SCHULINE,NC & 0 & NA & $\mathrm{B} / \mathrm{C} 2$ & $16-30$ & mixed $10 y r 5 / 1,5 / 6,5 / 8$ & sil loess & $\mathrm{cp}$ & firm & 6 & As \\
\hline 178 & SCHULINE,NC & 0 & NA & $\mathrm{B} / \mathrm{C} 3$ & $30-60$ & mixed $10 y r 5 / 6,5 / 2,5 / 1,5 / 8$ not cf & sil, sicl, cl & $\mathrm{cp}$ & firm & 6 & \\
\hline 179 & SCHULINE,NC & 2 & $\mathrm{~N}$ & AP & $0-6$ & $\operatorname{mix} 10 y r 4 / 2,5 / 1$ cf $10 y r 5 / 8$ & sil & $1 \mathrm{fsbk}$ & $\mathrm{fr}$ & 6.5 & As \\
\hline 179 & SCHULINE,NC & 2 & $\mathrm{~N}$ & $\mathrm{C} 1$ & $006-22$ & $\operatorname{mix} 2.5 y r 6 / 2,10 y r$ 4/2, 5/1;CMD 5/8 & sil & mass $\mathrm{cp}$ & $\mathrm{fr}$ & 6.5 & As \\
\hline 179 & SCHULINE,NC & 2 & $\mathrm{~N}$ & $\mathrm{C} 2$ & $22-31$ & $10 y r 5 / 1$ many $10 y r 5 / 8$ & sil & mass $\mathrm{cp}$ & $\mathrm{fr}$ & 6 & As \\
\hline 179 & SCHULINE,NC & 2 & $\mathrm{~N}$ & C3 & $31-60$ & $\operatorname{mix} 10 y r 5 / 8,5 / 1,2.5$ yr 6/2 & sil/sicl & mass $\mathrm{cp}$ & $\mathrm{fr}$ & 5.5 & NA \\
\hline 180 & LENZBURG & 3 & NW & AP & $0-2$ & $10 y r 4 / 2$ & sil & $1 \mathrm{fgr}$ & $\mathrm{fr}$ & 7 & as \\
\hline 180 & LENZBURG & 3 & NW & $\mathrm{B} / \mathrm{C} 1$ & $002-13$ & $\operatorname{mix} 10 y r 5 / 4,5 / 8,5 / 1$ & sil, sicl & $\mathrm{cp}, 2 \mathrm{fsbk}$ & $\mathrm{fr}$ & 7 & as \\
\hline 180 & LENZBURG & 3 & NW & $\mathrm{B} / \mathrm{C} 2$ & $13-30$ & $\operatorname{mix} 2.5 y r 6 / 2,10 y r 5 / 4,5 / 8$ & sicl & $\mathrm{cp}$ & firm & 5.5 & as \\
\hline 180 & LENZBURG & 3 & NW & $\mathrm{B} / \mathrm{C} 3$ & $30-43$ & $\operatorname{mix} 10 y r 5 / 1,5 / 8,5 / 4,5 / 8$ & $\mathrm{cl}, \mathrm{sicl}$ & $\mathrm{cp}, 2 \mathrm{fsbk}$ & $2 \%$ grav. Vfirm & $7.5, \mathrm{FC}$ & as \\
\hline 180 & LENZBURG & 3 & NW & $\mathrm{B} / \mathrm{C} 4$ & $43-62$ & $\operatorname{mix} 10 y r 5 / 1,5 / 8$ & $\mathrm{cl}$, till $\mathrm{cp}$ & $\mathrm{cp}$ & $5 \%$ grav. Vfirm & $8, \mathrm{FC}$ & NA \\
\hline 181 & SCHULINE,NC & 1 & $\mathrm{~N}$ & AP & $0-7$ & $\operatorname{mix} 10 y r 4 / 2,5 / 1$ cd $10 y r 5 / 8$ & sil & $1 \mathrm{fsbk}$ & $\mathrm{fr}$ & 6.5 & As \\
\hline 181 & SCHULINE,NC & 1 & $\mathrm{~N}$ & $\mathrm{~B} / \mathrm{C} 1$ & $007-28$ & $\operatorname{mix} 10 y r 5 / 4,75$ yr $4 / 6,5 / 1$ & cl 5\%ag till & $\mathrm{cp}$ & firm & 5.5 & As \\
\hline 181 & SCHULINE,NC & 1 & $\mathrm{~N}$ & $\mathrm{~B} / \mathrm{C} 2$ & $28-46$ & $\operatorname{mix} 2.5 \operatorname{Yr} 6 / 2,10 y r 5 / 4,5 / 8$ & sil, sicl & $\mathrm{cp}$ & firm & 5.5 & As \\
\hline 181 & SCHULINE,NC & 1 & $\mathrm{~N}$ & $\mathrm{~B} / \mathrm{C} 3$ & $46-60$ & $\operatorname{mix} 10 y r 5 / 6,2.5 y r 6 / 2,5 / 8$ & $\mathrm{cl}, \mathrm{sicl}$ & cp 5\% gr. & firm & 7 & NA \\
\hline 183 & SCHULINE & 2 & NA & $A$ & $0-6$ & NA & sil & $1 \mathrm{fgr}$ & $\mathrm{fr}$ & $7.5, \mathrm{FC}$ & As \\
\hline 183 & SCHULINE & 2 & NA & $A / B$ & $006-12$ & $10 y r 4 / 3 \quad 10 y r 4 / 6$ & sil & $1 \mathrm{fsbk}$ & $\mathrm{fi}$ & $7.5, \mathrm{FC}$ & As \\
\hline
\end{tabular}




\begin{tabular}{|c|c|c|c|c|c|c|c|c|c|c|c|}
\hline Site & Series & Slope & Aspect & Horizon & Depth & Moist Color & Texture & Structure & Consistency & Ph & Boundary \\
\hline 183 & SCHULINE & 2 & NA & $\mathrm{B} / \mathrm{C} 1$ & $012-48$ & 10yr 4/4 10yr4/2 10yr 4/6 & sicl & $\mathrm{cp}$ & vfi & $8, \mathrm{FC}$ & Cs \\
\hline 183 & SCHULINE & 2 & NA & $\mathrm{B} / \mathrm{C} 2$ & $48-60$ & N 2/0 5 yr $5 / 1$ mix coal & $\mathrm{cl}$ & $\mathrm{m}$ & & $8, \mathrm{FC}$ & NA \\
\hline 184 & SCHULINE & 1 & NA & A & $0-8$ & $10 y r 4 / 3$ & sil & $1 \mathrm{mgn}$ & $\mathrm{fr}$ & 5.5 & As \\
\hline 184 & SCHULINE & 1 & NA & $\mathrm{B} / \mathrm{C} 1$ & $008-20$ & $10 y r 4 / 3 \mathrm{cp} 10 y r 4 / 6$ & sicl & $1 \mathrm{tkpl}$ & vfi & $8, \mathrm{FC}$ & Cs \\
\hline 184 & SCHULINE & 1 & NA & $\mathrm{B} / \mathrm{C} 2$ & $20-46$ & $10 \mathrm{yr} 4 / 1 \mathrm{cp} \mathrm{10yr} \mathrm{4/6}$ & sicl & fvcp & efi & $8, \mathrm{FC}$ & Cs \\
\hline 184 & SCHULINE & 1 & NA & $\mathrm{B} / \mathrm{C} 3$ & $46-60$ & $10 y r 5 / 6$ cp $2.5 y r 5 / 1$ & $\mathrm{cl}$ & $\mathrm{m}$ & efi & $8, F C$ & NA \\
\hline 185 & SCHULINE & 0 & NA & A & $0-7$ & 10yr 3/3 (med dark) & sil & $2 \mathrm{mgr}$ & $\mathrm{fr}$ & 6.6 & As \\
\hline 185 & SCHULINE & 0 & NA & $\mathrm{B} / \mathrm{C} 1$ & $007-24$ & $10 y r 5 / 3 c p \quad 10 y r 5 / 6$ & sicl & $1 \mathrm{tkpl}$ & fi & $8, \mathrm{FC}$ & Cs \\
\hline 185 & SCHULINE & 0 & NA & $\mathrm{B} / \mathrm{C} 2$ & $24-46$ & $10 y r 5 / 62.5 Y r 3 / 1$ & sicl & fvcp & vfi & $8, \mathrm{FC}$ & Cs \\
\hline 185 & SCHULINE & 0 & NA & $\mathrm{B} / \mathrm{C} 3$ & $46-60$ & $10 y r 5 / 12.5$ yr $5 / 1$ & I & mass & efi & $8, \mathrm{FC}$ & NA \\
\hline 186 & SCHULINE & 1 & NA & A & $0-8$ & $10 y r 4 / 3$ & sil & $1 \mathrm{mgr}$ & $\mathrm{fr}$ & $7.6, \mathrm{FC}$ & As \\
\hline 186 & SCHULINE & 1 & NA & $\mathrm{B} / \mathrm{C} 1$ & $008-22$ & $10 y r 5 / 1$ ср 10yr 4/6 & sil & $2 \mathrm{tkpl}$ & $\mathrm{fi}$ & $8, \mathrm{FC}$ & Cs \\
\hline 186 & SCHULINE & 1 & NA & $\mathrm{B} / \mathrm{C} 2$ & $22-44$ & $10 y r 5 / 8,10 y r 5 / 1$ & sicl & fvcp & $n f i$ & $8, \mathrm{FC}$ & Cs \\
\hline 186 & SCHULINE & 1 & NA & $\mathrm{B} / \mathrm{C} 3$ & $44-60$ & 10yr 5/8 10yr 5/1 & $\mathrm{cl}$ & mass & efi & $8, \mathrm{FC}$ & NA \\
\hline 187 & SCHULINE & 1 & NA & A & $0-12$ & 10 yr $5 / 3$ & sil & $1 \mathrm{mgr}$ & efi & 5.5 & As \\
\hline 187 & SCHULINE & 1 & NA & $\mathrm{B} / \mathrm{C} 1$ & $012-20$ & 10yr 5/6 CP 2.5 yr 6/2 & sicl & $2 \mathrm{tkpl}$ & epi & $8, \mathrm{FC}$ & Cs \\
\hline 187 & SCHULINE & 1 & NA & $\mathrm{B} / \mathrm{C} 2$ & $20-48$ & $10 y r 5 / 82.55 / 1$ & sicl & fvcp & efi & $8, \mathrm{FC}$ & Cs \\
\hline 187 & SCHULINE & 1 & NA & $\mathrm{B} / \mathrm{C} 3$ & $48-60$ & 7.5 yr $4 / 42.5$ yr $5 / 1$ w/coal & chcl & $\mathrm{m}$ & efi & $8, \mathrm{FC}$ & NA \\
\hline
\end{tabular}




\begin{tabular}{|c|c|c|c|c|c|c|c|c|c|c|c|}
\hline Site & Series & Slope & Aspect & Horizon & Depth & Moist Color & Texture & Structure & Consistency & $\mathbf{P h}$ & Boundary \\
\hline 188 & LENZBURG & 0 & NA & $A$ & $0-12$ & $10 y r 5 / 6$ & sicl & fvcp & vfi & $8, \mathrm{FC}$ & Cs \\
\hline 188 & LENZBURG & 0 & NA & $\mathrm{B} / \mathrm{C} 1$ & $012-36$ & $10 y r 5 / 410 y r 5 / 1 \quad 10 y r 5 / 6$ & sicl & fvcp & vfi & $8, F C$ & Cs \\
\hline 188 & LENZBURG & 0 & NA & $\mathrm{B} / \mathrm{C} 2$ & $36-60$ & $10 y r 5 / 1,10 y r 5 / 6$ some coal & chch & $\mathrm{m}$ & vfi & $8, \mathrm{FC}$ & NA \\
\hline 189 & SCHULINE & 2 & NA & Ap & $0-9$ & $10 Y r 5 / 4$ & sil & $2 \mathrm{mgr}$ & $\mathrm{fr}$ & 5.5 & As \\
\hline 189 & SCHULINE & 2 & NA & $\mathrm{B} / \mathrm{C} 1$ & 009-16 & $10 y r 5 / 6 \quad 10 y r 4 / 3$ & sicl & 1VCP & vfi & $8, \mathrm{FC}$ & As \\
\hline 189 & SCHULINE & 2 & NA & $\mathrm{B} / \mathrm{C} 2$ & $16-46$ & $10 y r 5 / 610 y r 4 / 3$ & sicl & $1 \mathrm{vcp}$ & efi & $8, \mathrm{FC}$ & Cs \\
\hline 189 & SCHULINE & 2 & NA & $\mathrm{B} / \mathrm{C} 3$ & $46-60$ & 10 yr $5 / 1$ & $\mathrm{ch} \mathrm{cl}$ & $\mathrm{m}$ & efi & $8, \mathrm{FC}$ & NA \\
\hline 190 & LENZBURG & 16 & NA & Ap & $0-8$ & $10 y r 5 / 3$ & sil & $2 \mathrm{mgr}$ & $\mathrm{fr}$ & $8, \mathrm{FC}$ & As \\
\hline 190 & LENZBURG & 16 & NA & $\mathrm{B} / \mathrm{C} 1$ & $008-36$ & $10 y r 5 / 6$ & sicl & fvcp & efi & $8, \mathrm{FC}$ & Cs \\
\hline 190 & LENZBURG & 16 & NA & $\mathrm{B} / \mathrm{C} 2$ & $36-60$ & 2.5 yr 5/1 10yr 5/4 mix coal & ch I & $\mathrm{m}$ & efi & $8, \mathrm{FC}$ & NA \\
\hline 191 & SCHULINE & 2 & $\mathrm{~N}$ & AP & $0-6$ & $\operatorname{mix} 10 y r 4 / 2,5 / 6(10 \%)$ & sil, sicl & $1 \mathrm{fgr}$ & $\mathrm{fr}$ & 7 & As \\
\hline 191 & SCHULINE & 2 & $\mathrm{~N}$ & $\mathrm{~B} / \mathrm{C} 1$ & 006-18 & $\operatorname{mix} 10 y r 5 / 4,5 / 8,5 / 2$ & cl $5 \%$ & $\mathrm{cp}$ & vfirm & 7 & As \\
\hline 191 & SCHULINE & 2 & $\mathrm{~N}$ & $\mathrm{~B} / \mathrm{C} 2$ & $18-34$ & $\operatorname{mix} 10 y r 5 / 1,5 / 8$ & cl 5\% & $\mathrm{cp}$ & vfirm & $7.5, \mathrm{FC}$ & As \\
\hline 191 & SCHULINE & 2 & $\mathrm{~N}$ & $\mathrm{~B} / \mathrm{C} 3$ & $34-55$ & $\operatorname{mix} 10 y r 6 / 2,10 y r 5 / 8,4 / 2$ & cl $5 \%$ & $\mathrm{cp}$ & vfirm & $7.5, \mathrm{FC}$ & As \\
\hline 191 & SCHULINE & 2 & $\mathrm{~N}$ & $\mathrm{~B} / \mathrm{C} 4$ & $55-60$ & $\operatorname{mix} 10 y r 6 / 2,10 y r 5 / 8,4 / 2$ & cl $5 \%$ & $\mathrm{cp}$ & vfirm & $7.5, \mathrm{FC}$ & As \\
\hline 192 & HOSMER & 4 & $\mathrm{~N}$ & $A$ & $0-6$ & $10 y r 4 / 2$ & sil & $2 \mathrm{fgr}$ & $\mathrm{fr}$ & 6 & As \\
\hline 192 & HOSMER & 4 & $\mathrm{~N}$ & $E$ & 006-11 & $10 y r 5 / 4$ & sil & $2 \mathrm{fsbk}$ & $\mathrm{fr}$ & 5.5 & Cs \\
\hline 192 & HOSMER & 4 & $\mathrm{~N}$ & $\mathrm{Bt} 1$ & $011-20$ & $10 \mathrm{yr} 5 / 6,4 / 4 \mathrm{cfompf}$ & sil & $2 m s b k$ & $\mathrm{fr}$ & 5.5 & Cs \\
\hline
\end{tabular}




\begin{tabular}{|c|c|c|c|c|c|c|c|c|c|c|c|}
\hline Site & Series & Slope & Aspect & Horizon & Depth & Moist Color & Texture & Structure & Consistency & $\mathrm{Ph}$ & Boundary \\
\hline 192 & HOSMER & 4 & $\mathrm{~N}$ & $\mathrm{Bt} 2 / \mathrm{E}$ & $20-30$ & B-10yr5/6 w 5/2, 5/8M 4/8; E,7/2 & sicl & $2 m s b k$ & firm & 5.5 & Cs \\
\hline 192 & HOSMER & 4 & $\mathrm{~N}$ & Bt1 & $30-50$ & 10yr $5 / 4$, few $5 / 2,5 / 8 ; E, 7 / 2$ & sicl & $2 \mathrm{msbk}$ & firm & 5.5 & Cs \\
\hline 192 & HOSMER & 4 & $\mathrm{~N}$ & Bt2 & $50-60$ & $10 \mathrm{yr} 5 / 6$ cfom $5 / 2,5 / 8,10 \mathrm{yr} 7 / 2 \mathrm{cf}$ & sil & $1 \mathrm{msk}$ & fri & 6 & NA \\
\hline 195 & SCHULINE & 3 & NA & AP & $0-4$ & $10 y r 4 / 2$ & sil & $1 \mathrm{fgr}$ & $\mathrm{fr}$ & 6.5 & As \\
\hline 195 & SCHULINE & 3 & NA & $\mathrm{B} / \mathrm{C} 1$ & $004-7$ & $\operatorname{mix} 2.5 \mathrm{YR} 6 / 2,5 / 8$ & CL 5\% & $\mathrm{cp}$ & firm & 5.5 & As \\
\hline 195 & SCHULINE & 3 & NA & $\mathrm{B} / \mathrm{C} 2$ & $007-30$ & $\operatorname{mix} 7.5 \mathrm{yr} 4 / 6,10 y r 5 / 4$ & CL 5\% & $\mathrm{cp}$ & firm & $7.5, \mathrm{FC}$ & As \\
\hline 195 & SCHULINE & 3 & NA & $\mathrm{B} / \mathrm{C} 3$ & $30-80$ & 7.5 yr. $5 / 8$ till & $5 \% \mathrm{cl}$ & $\mathrm{cp}$ compl & firm & $7.5, \mathrm{FC}$ & NA \\
\hline 196 & SWANWICK & 2 & NA & A & $0-5$ & $10 y r 5 / 3$ & sil & $2 \mathrm{mgr}$ & $\mathrm{fr}$ & 7.6,FC & As \\
\hline 196 & SWANWICK & 2 & NA & $\mathrm{B} / \mathrm{C} 1$ & $005-12$ & $10 y r 5 / 2$ cd $10 y r 5 / 6$ & sil & $2 \mathrm{msbk}$ & fr & $7.8, \mathrm{FC}$ & Cs \\
\hline 196 & SWANWICK & 2 & NA & $\mathrm{B} / \mathrm{C} 2$ & $012-24$ & $10 y r 5 / 210 y r 5 / 4$ & sicl & $2 \mathrm{mabk}$ & $\mathrm{fi}$ & $8, \mathrm{FC}$ & Cs \\
\hline 196 & SWANWICK & 2 & NA & $\mathrm{B} / \mathrm{C} 3$ & $24-44$ & $10 y r 5 / 6,10 y r 4 / 2$ & sicl & 3 mabk & vfi & $8, \mathrm{FC}$ & Cs \\
\hline 196 & SWANWICK & 2 & NA & $\mathrm{B} / \mathrm{C} 4$ & $44-60$ & 10yr 4/2 10yr5/6 mix with coal & sicl & $2 \mathrm{mabk}$ & efi & $7.8 \mathrm{FC}$ & NA \\
\hline 198 & SCHULINE & 1 & NA & A & $0-11$ & $10 y r 4 / 4,10 y r 5 / 1$ & sil & $1 \mathrm{fgr}$ & $\mathrm{fr}$ & $8, \mathrm{FC}$ & As \\
\hline 198 & SCHULINE & 1 & NA & $\mathrm{B} / \mathrm{C} 1$ & $011-20$ & $10 y r 4 / 2,10 y r 5 / 4$ & sil & few vcp & vfi & $8, \mathrm{FC}$ & As \\
\hline 198 & SCHULINE & 1 & NA & $\mathrm{B} / \mathrm{C} 2$ & $20-36$ & $10 y r 4 / 2,10 y r 5 / 6$ & sicl & few vcp & efi & $8, \mathrm{FC}$ & As \\
\hline 198 & SCHULINE & 1 & NA & $\mathrm{B} / \mathrm{C} 3$ & $36-56$ & $10 y r 5 / 6,10 y r 5 / 1,10 y r 5 / 4$ & sicl & few vcp & efi & $8, \mathrm{FC}$ & As \\
\hline 198 & SCHULINE & 1 & NA & $\mathrm{B} / \mathrm{C} 4$ & $56-60$ & $10 \mathrm{yr} 5 / 6,2.5 \mathrm{yr} /$ some coal & $\mathrm{ch} \mathrm{cl}$ & massive & efi & $8, \mathrm{FC}$ & NA \\
\hline 199 & SCHULINE & 4 & NA & Ap & $0-9$ & $10 y r 4 / 6$ & sil & $1 \mathrm{mgr}$ & vfi & $8, F C$ & As \\
\hline
\end{tabular}




\begin{tabular}{|c|c|c|c|c|c|c|c|c|c|c|c|}
\hline Site & Series & Slope & Aspect & Horizon & Depth & Moist Color & Texture & Structure & Consistency & $\mathbf{P h}$ & Boundary \\
\hline 199 & SCHULINE & 4 & NA & $\mathrm{B} / \mathrm{C} 1$ & $009-17$ & $10 y r 5 / 8,10 y r 5 / 6$ & sicl & fvcp & efi & 8.,FC2 & As \\
\hline 199 & SCHULINE & 4 & NA & $\mathrm{B} / \mathrm{C} 2$ & $17-32$ & $10 y r 5 / 4,10 y r 5 / 2$ & sicl & fvcp & efi & $8, \mathrm{FC}$ & Cs \\
\hline 199 & SCHULINE & 4 & NA & $\mathrm{B} / \mathrm{C} 3$ & $32-47$ & $10 \mathrm{yr} 5 / 4,10 \mathrm{yr} 5 / 1$ some coal & sicl & fvcp & efi & $8, \mathrm{FC}$ & Cs \\
\hline 199 & SCHULINE & 4 & NA & $\mathrm{B} / \mathrm{C} 4$ & $47-60$ & $10 y r 5 / 4,7.5 y r 5 / 6$ coal & $\mathrm{ch} \mathrm{cl}$ & massive & efi & $8, \mathrm{FC}$ & NA \\
\hline 200 & SWANWICK & 1 & NA & Ap & $0-8$ & $10 y r 5 / 4$ & Sil & $1 \mathrm{mgr}$ & $\mathrm{Fr}$ & 5.5 & As \\
\hline 200 & SWANWICK & 1 & NA & $\mathrm{B} / \mathrm{C} 1$ & $008-17$ & $10 y r 5 / 6$ & Sicl & fvcp & Vfi & 5.6 & Cs \\
\hline 200 & SWANWICK & 1 & NA & $\mathrm{B} / \mathrm{C} 2$ & $17-30$ & $10 y r 5 / 6,10 y r 5 / 4$ & Sicl & fvcp & Efi & 5.8 & Cs \\
\hline 200 & SWANWICK & 1 & NA & $\mathrm{B} / \mathrm{C} 3$ & $30-42$ & $10 y r 5 / 8,10 y r 5 / 6$ & Sicl & $f v c p$ & Efi & 6.4 & Cs \\
\hline 200 & SWANWICK & 1 & NA & $\mathrm{B} / \mathrm{C} 4$ & $42-60$ & $10 y r 5 / 8,2.5 y r 6 / 1$ coal mix & $\mathrm{Cl}, \mathrm{L}$ & $\mathrm{m}$ & Efi & $7.8, \mathrm{FC}$ & NA \\
\hline 201 & SCHULINE & 2 & NA & Ap & $0-7$ & $10 y r 5 / 4$ & Sil & $2 m g r$ & $\mathrm{Fr}$ & 5 & As \\
\hline 201 & SCHULINE & 2 & NA & $\mathrm{B} / \mathrm{C} 1$ & $007-15$ & $10 y r 5 / 6$ & Sicl & $2 \mathrm{tkpl}$ & Vfi & 6.6 & Cs \\
\hline 201 & SCHULINE & 2 & NA & $\mathrm{B} / \mathrm{C} 2$ & $15-24$ & $10 y r 5 / 3,10 y r 5 / 4$ & Sicl & 2tkpl & Vfi & 7.6,FC & Cs \\
\hline 201 & SCHULINE & 2 & NA & $\mathrm{B} / \mathrm{C} 3$ & $24-42$ & $10 y r 5 / 6,2.5 y r 4 / 4$ & Sicl & fvcp & Efi & 7.4 & Cs \\
\hline 201 & SCHULINE & 2 & NA & $\mathrm{B} / \mathrm{C} 3$ & $42-60$ & $10 y r 6 / 2,7.5 y r 4 / 4$ & $\mathrm{Cl}, \mathrm{L}$ & $\mathrm{m}$ & Efi & 7.6,FC & NA \\
\hline 202 & SCHULINE & 1 & NA & Ap & $0-9$ & $10 y r 5 / 3$ & Sil & $2 \mathrm{mgr}$ & $\mathrm{Fr}$ & 5.5 & As \\
\hline 202 & SCHULINE & 1 & NA & $\mathrm{B} / \mathrm{C} 1$ & $009-16$ & cf $10 y r 5 / 2,10 y r 5 / 1$ & Sicl & fvcp & Vfi & $8, \mathrm{FC}$ & Cs \\
\hline 202 & SCHULINE & 1 & NA & $\mathrm{B} / \mathrm{C} 2$ & $16-48$ & cf $10 y r 5 / 3,10 y r 5 / 4$ & Sicl & fvcp & Efi & $8, \mathrm{FC}$ & Cs \\
\hline 202 & SCHULINE & 1 & NA & $\mathrm{B} / \mathrm{C} 3$ & $48-60$ & $10 y r 5 / 3$ cf $10 y r 5 / 6$ some coal/sh & Ch, L & $\mathrm{m}$ & Efi & $8, \mathrm{FC}$ & NA \\
\hline
\end{tabular}




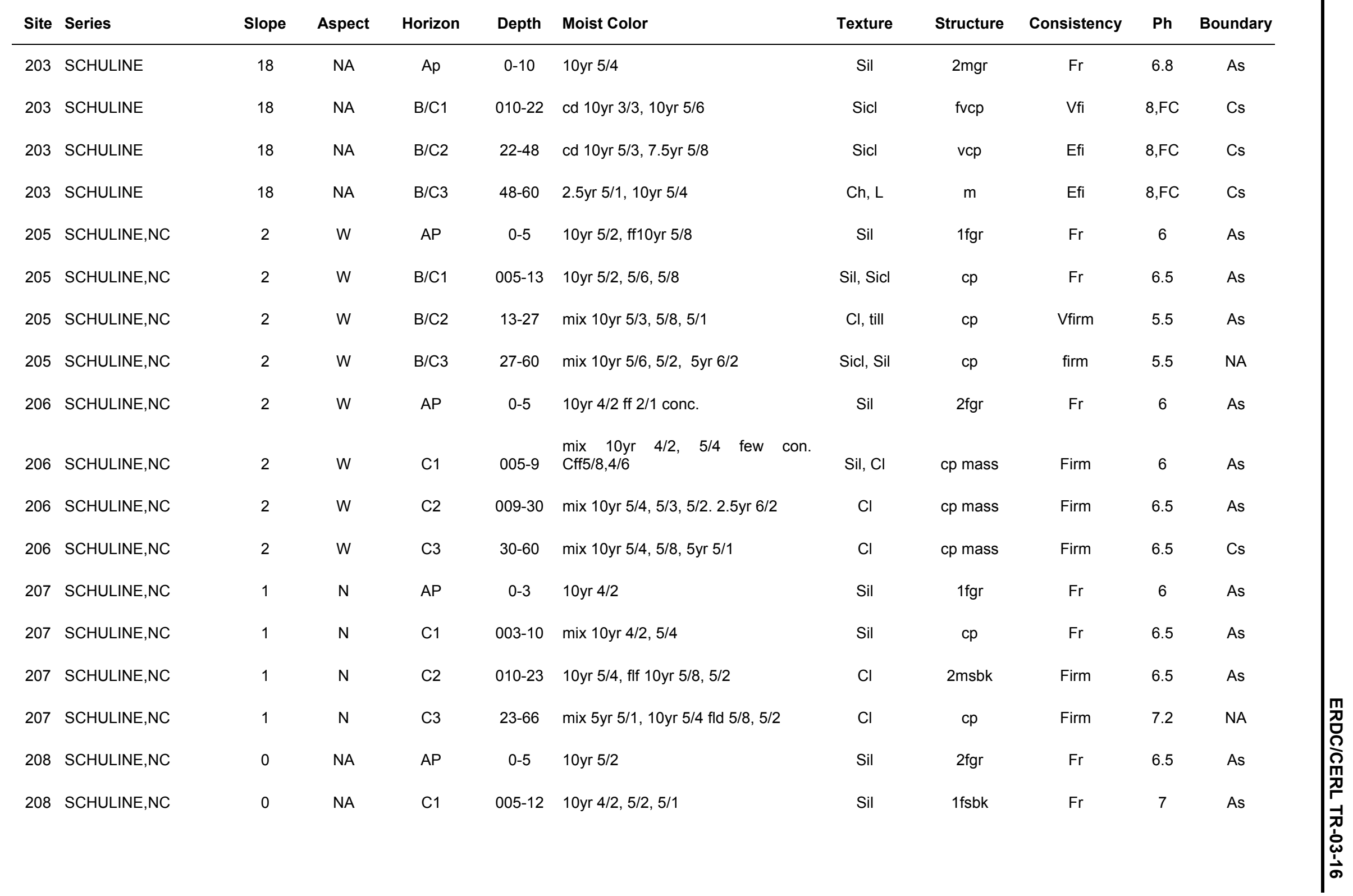




\begin{tabular}{|c|c|c|c|c|c|c|c|c|c|c|c|}
\hline Site & Series & Slope & Aspect & Horizon & Depth & Moist Color & Texture & Structure & Consistency & $\mathbf{P h}$ & Boundary \\
\hline 208 & SCHULINE,NC & 0 & NA & $\mathrm{C} 2$ & $012-23$ & 10yr 5/2, CMD 10yr 5/8, 2.5y 6/2 & Cl Till & $1 \mathrm{msbk}$ & Firm & 7 & As \\
\hline 208 & SCHULINE,NC & 0 & NA & C3 & $23-38$ & $2.5 \mathrm{yr} 6 / 2,10 \mathrm{yr} 5 / 4,4 / 1$ & Sil loess & cp mass & Firm & 5.5 & As \\
\hline 208 & SCHULINE,NC & 0 & NA & $\mathrm{C} 4$ & $38-60$ & $\operatorname{mix} 10 y r 5 / 4,5 / 8,2.5 y r 6 / 2$ & $\mathrm{Cl}$ Till & $\mathrm{cp}$ mass & Firm & 7 & NA \\
\hline 209 & SCHULINE & 12 & SW & $A$ & $0-7$ & $\operatorname{mix} 10 y r 4 / 2,5 / 6$ & Sil & $1 \mathrm{fgr}$ & $\mathrm{Fr}$ & 6 & As \\
\hline 209 & SCHULINE & 12 & sW & $\mathrm{C} 1$ & $007-16$ & $\operatorname{mix} 10 y r 5 / 6,4 / 2$ & Sil, Sicl & $\mathrm{cp}, 1 \mathrm{fsbk}$ & $\mathrm{Fr}$ & 5.5 & As \\
\hline 209 & SCHULINE & 12 & SW & $\mathrm{C} 2$ & $16-25$ & $\operatorname{mix} 10 y r 5 / 6,5 / 2,4 / 4,4 / 6$ & Sicl & $\mathrm{cp}, 2 \mathrm{msbk}$ & $\mathrm{Fr}$ & $7.5, \mathrm{FC}$ & As \\
\hline 209 & SCHULINE & 12 & SW & $\mathrm{C} 3$ & $25-42$ & $\operatorname{mix} 7.5 y r 4 / 4,10 y r 5 / 6$ & Sil & $1 \mathrm{msbk}$ & $\mathrm{Fr}$ & $7.5, \mathrm{FC}$ & As \\
\hline 209 & SCHULINE & 12 & SW & $\mathrm{C} 4$ & $42-62$ & $\operatorname{mix} 10 y r 5 / 6,5 / 1$, fow $4 / 6$ & $\mathrm{Cl}$ & $\mathrm{cp}$ till struc & Firm & $7.5, \mathrm{FC}$ & NA \\
\hline 207 & SCHULINE & 1 & NA & $A$ & $0-5$ & $10 y r 4 / 3$ & Sil & $1 \mathrm{fgr}$ & $\mathrm{Fr}$ & 7 & As \\
\hline 207 & SCHULINE & 1 & NA & $\mathrm{B} / \mathrm{C} 1$ & 005-19 & $10 y r 4 / 4,10 y r 4 / 6$ & Sicl & $1 \mathrm{fsbk}$ & $\mathrm{Fi}$ & $8, \mathrm{FC}$ & Cs \\
\hline 207 & SCHULINE & 1 & NA & $\mathrm{B} / \mathrm{C} 2$ & $19-50$ & $10 y r 5 / 1,10 y r 4 / 3,10 y r 4 / 6$ & Sicl & $\mathrm{cp}$ & Vfi & $8, \mathrm{FC}$ & Cs \\
\hline 207 & SCHULINE & 1 & NA & $\mathrm{B} / \mathrm{C} 3$ & $50-60$ & $10 y r 5 / 610 y r 5 / 1$ & $\mathrm{Cl}$ & $\mathrm{m}$ & Efi & $8, \mathrm{FC}$ & NA \\
\hline 210 & SCHULINE & 1 & NA & $A$ & $0-5$ & $10 y r 4 / 3$ & Sil & $1 \mathrm{fgr}$ & $\mathrm{Fr}$ & 7 & As \\
\hline 210 & SCHULINE & 1 & NA & $\mathrm{BC}$ & 005-19 & $10 y r 4 / 410 y r 4 / 6$ & Sicl & $1 \mathrm{fsbk}$ & $\mathrm{Fi}$ & 8 & Cs \\
\hline 210 & SCHULINE & 1 & NA & $\mathrm{BC} 2$ & $19-50$ & $10 y r 5 / 110 y r 4 / 310 y r$ 4/6 & Sicl & $\mathrm{Cp}$ & $V f i$ & 8 & Cs \\
\hline 210 & SCHULINE & 1 & NA & NA & $50-60$ & $10 y r 5 / 610 y r 5 / 1$ & $\mathrm{Cl}$ & M & Efi & 8 & NA \\
\hline 211 & LENZBURG & 11 & NA & $A$ & $0-12$ & $10 y r 5 / 3,10 y r 5 / 2$ & Sil & $1 \mathrm{fgr}$ & $\mathrm{Fr}$ & 6 & As \\
\hline 211 & LENZBURG & 11 & NA & $\mathrm{B} / \mathrm{C} 1$ & $012-20$ & $10 y r 4 / 1,10 y r 5 / 2,10 y r 5 / 4$ & Sicl & $\mathrm{cp}$ & $\mathrm{Fi}$ & $7.5, \mathrm{FC}$ & Cs \\
\hline
\end{tabular}




\begin{tabular}{|c|c|c|c|c|c|c|c|c|c|c|c|}
\hline Site & Series & Slope & Aspect & Horizon & Depth & Moist Color & Texture & Structure & Consistency & $\mathbf{P h}$ & Boundary \\
\hline 211 & LENZBURG & 11 & NA & $\mathrm{C}$ & $20-60$ & $10 y r 4 / 4$ coal, $10 y r 4 / 2$ & $\mathrm{Cl}$ & $\mathrm{m}$ & Efi & $8, \mathrm{FC}$ & NA \\
\hline 212 & SCHULINE & 2 & NA & Ap & $0-8$ & $10 y r 4 / 3$ & Sil & $2 \mathrm{mgr}$ & $\mathrm{Fr}$ & $8, \mathrm{FC}$ & As \\
\hline 212 & SCHULINE & 2 & NA & $\mathrm{B} / \mathrm{C} 1$ & $008-13$ & $10 y r 5 / 6,10 y r 5 / 2$ & Sil & 2 mabk & $\mathrm{Fr}$ & $8, \mathrm{FC}$ & As \\
\hline 212 & SCHULINE & 2 & NA & $\mathrm{B} / \mathrm{C} 2$ & $13-27$ & $10 y r 5 / 2$ cd $10 y r 5 / 4$ & Sicl & $2 \mathrm{mabk}$ & $\mathrm{Fi}$ & $8, \mathrm{FC}$ & Cs \\
\hline 212 & SCHULINE & 2 & NA & $\mathrm{B} / \mathrm{C} 3$ & $27-40$ & $10 y r 5 / 6,10 y r 5 / 2$ & Sicl & $2 m a b k$ & Vfi & $8, \mathrm{FC}$ & AS \\
\hline 212 & SCHULINE & 2 & NA & $\mathrm{B} / \mathrm{C} 4$ & $40-60$ & $10 y r 5 / 6,10 y r 5 / 1$ mix coal & Sicl & 2 mabk & Efi & $8 \mathrm{FC}$ & NA \\
\hline 213 & SCHULINE & 2 & NA & A & $0-3$ & $10 y r 4 / 3$ & Sil & $1 \mathrm{fgr}$ & $\mathrm{Fr}$ & $7.5, \mathrm{FC}$ & As \\
\hline 213 & SCHULINE & 2 & NA & $\mathrm{A} / \mathrm{E}$ & $003-9$ & $10 y r 4 / 6,10 y r 4 / 3$ & Sil & $1 \mathrm{fgr}$ & $\mathrm{Fr}$ & $7.8, \mathrm{FC}$ & As \\
\hline 213 & SCHULINE & 2 & NA & $\mathrm{B} / \mathrm{C} 1$ & $009-24$ & $10 y r 5 / 4,10 y r 4 / 6$ & Sicl & $\mathrm{cp}$ & Vfi & $8, \mathrm{FC}$ & Cs \\
\hline 213 & SCHULINE & 2 & NA & $\mathrm{B} / \mathrm{C} 2$ & $24-44$ & $5 \% 10 y r 5 / 1,10 y r 4 / 6,10 y r 5 / 4$ & Sicl & $\mathrm{cp}$ & Vfi & $8, \mathrm{FC}$ & AS \\
\hline 213 & SCHULINE & 2 & NA & $\mathrm{B} / \mathrm{C} 3$ & $44-60$ & coal mix $10 y r$ 4/2 & $\mathrm{Cl}$ & $\mathrm{m}$ & Efi & $8, \mathrm{FC}$ & NA \\
\hline 214 & SCHULINE & 1 & NA & Ap & $0-4$ & $10 y r 4 / 5$ & Sil & $1 \mathrm{fgr}$ & $\mathrm{Fr}$ & 7 & As \\
\hline 214 & SCHULINE & 1 & NA & $\mathrm{B} / \mathrm{C} 1$ & $004-9$ & $10 y r 4 / 4$ & Sil & $1 \mathrm{fgr}$ & $\mathrm{Fr}$ & $7.5, \mathrm{FC}$ & As \\
\hline 214 & SCHULINE & 1 & NA & $\mathrm{B} / \mathrm{C} 2$ & $009-16$ & $10 y r 5 / 3,10 y r$ 4/6 & Sicl & $\mathrm{cp}$ & $\mathrm{Fi}$ & $7.5, \mathrm{FC}$ & As \\
\hline 214 & SCHULINE & 1 & NA & $\mathrm{B} / \mathrm{C} 3$ & $16-60$ & $2.5 y r 5 / 1,10 y r$ 4/6 N2/0 mix coal & $\mathrm{Cl}$ & $\mathrm{m}$ & $\mathrm{Fi}$ & 4.5 & NA \\
\hline 216 & LENZBURG & 28 & NA & A & $0-5$ & NA & Sil & $1 \mathrm{fgr}$ & $\mathrm{Fr}$ & 6 & As \\
\hline 216 & LENZBURG & 28 & NA & $\mathrm{B} / \mathrm{C}$ & $005-60$ & $2.5 y r 2 / 1,10 y r 5 / 6 \quad N 2 / 0$ & $\mathrm{Cl}$ & $\mathrm{m}$ & Efi & 8,FC & NA \\
\hline 217 & SCHULINE & 3 & NA & Ap & $0-4$ & $10 y r 4 / 3$ & Sil & $1 \mathrm{fgr}$ & $\mathrm{Fr}$ & $8, F C$ & As \\
\hline
\end{tabular}




\begin{tabular}{|c|c|c|c|c|c|c|c|c|c|c|c|}
\hline Site & Series & Slope & Aspect & Horizon & Depth & Moist Color & Texture & Structure & Consistency & $\mathrm{Ph}$ & Boundary \\
\hline 217 & SCHULINE & 3 & NA & $\mathrm{B} / \mathrm{C} 1$ & $004-12$ & $10 y r 4 / 4,10 y r 4 / 6$ & Sil & $1 \mathrm{fsbk}$ & $\mathrm{Fi}$ & $8, \mathrm{FC}$ & As \\
\hline 217 & SCHULINE & 3 & NA & $\mathrm{B} / \mathrm{C} 2$ & $012-22$ & $10 y r 5 / 3,10 y r 4 / 6$ & Sicl & $\mathrm{cp}$ & Vfi & $8, \mathrm{FC}$ & Cs \\
\hline 217 & SCHULINE & 3 & NA & $\mathrm{B} / \mathrm{C} 3$ & $22-60$ & $2.5 y r 5 / 1,5 G Y 5 / 1, N 2 / 0,10 y r 5 / 6$ & $\mathrm{Cl}$ & $\mathrm{m}$ & Efi & $8 \mathrm{FC}$ & NA \\
\hline 218 & SCHULINE & 2 & NA & Ap & $0-8$ & $10 y r 4 / 3$ & Sil & $1 \mathrm{fgr}$ & $\mathrm{Fr}$ & 6 & As \\
\hline 218 & SCHULINE & 2 & NA & $\mathrm{B} / \mathrm{C} 1$ & $008-16$ & $2.5 y 5 / 1,10 y r 4 / 4,10 y r 4 / 6$ & Sicl & $\mathrm{cp}$ & $\mathrm{Fi}$ & 5.5 & As \\
\hline 218 & SCHULINE & 2 & NA & $\mathrm{B} / \mathrm{C} 2$ & $16-40$ & $2.5 y 5 / 1,10 y r 5 / 4,10 y r 5 / 6$ & Sicl & $\mathrm{cp}$ & Vfi & 7.,FC5 & Cs \\
\hline 218 & SCHULINE & 2 & NA & $\mathrm{B} / \mathrm{C} 3$ & $40-60$ & $5 \mathrm{yr} 5 / 1, \mathrm{~N} 2 / 0$ coal $2.5 \mathrm{yr} 4 / 2$ & $\mathrm{Cl}$ & $\mathrm{m}$ & Efi & 7.,FC5 & NA \\
\hline 219 & SCHULINE & 8 & NA & $\mathrm{AP}$ & $0-9$ & $\operatorname{mix} 10 y r 4 / 290 \%, 5 / 610 \%$ & Sil $5 \%$ & $1 \mathrm{fgr}$ & $\mathrm{Fr}$ & 6.5 & As \\
\hline 219 & SCHULINE & 8 & NA & $\mathrm{B} / \mathrm{C} 1$ & 009-19 & $\operatorname{mix} 10 y r 5 / 4,5 / 8,5 / 1$ & $\mathrm{Cl}$ & $\mathrm{cp}$ & Firm & 7.,FC5 & As \\
\hline 219 & SCHULINE & 8 & NA & $\mathrm{B} / \mathrm{C} 2$ & $19-38$ & $\operatorname{mix} 10 y r 5 / 6,5 / 1$ ff $10 y r 5 / 8$ & $\mathrm{Cl}$ & $\mathrm{cp}$ & Vfirm & 7.,FC5 & As \\
\hline 219 & SCHULINE & 8 & NA & $\mathrm{B} / \mathrm{C} 3$ & $38-60$ & $\operatorname{mix} 10 y r 5 / 4,5 y r 5 / 8,10 y r 5 / 8$ & $\mathrm{Cl}$ & $\mathrm{cp}$ & Vfirm & 7.,FC5 & NA \\
\hline 220 & SCHULINE & 1 & NA & AP & $0-6$ & $10 y r 4 / 2$ & Sil & $2 \mathrm{fsbk}$ & $\mathrm{Fr}$ & 6.5 & As \\
\hline 220 & SCHULINE & 1 & NA & $\mathrm{C} 1$ & $006-11$ & $10 y r 4 / 2,5 / 6$ & Sil, Cl & $1 \mathrm{msbk} \mathrm{cp}$ & $\mathrm{Fr}$ & 7 & As \\
\hline 220 & SCHULINE & 1 & NA & $\mathrm{C} 2$ & 011-18 & $10 y r 5 / 6$, w $10 y r$ r $4 / 4$ & Sil & $1 \mathrm{msbk} \mathrm{cp}$ & Firm & 7 & As \\
\hline 220 & SCHULINE & 1 & NA & C3 & $18-39$ & $10 y r 4 / 4,10 y r 6 / 2,5 / 8$ & $\mathrm{Cl}$ & $2 \mathrm{msbk}, \mathrm{cp}$ & Firm & 7 & As \\
\hline 220 & SCHULINE & 1 & NA & $\mathrm{C} 4$ & $39-60$ & $10 y r 5 / 6,5 / 1,4 / 4$ & $\mathrm{Cl}$ & mass $\mathrm{cp}$ & Firm & $7.5, \mathrm{FC}$ & NA \\
\hline 221 & SCHULINE & 0 & NA & AP & $0-5$ & $10 y r 5 / 2$ & Sil & $2 f s b k$ & $\mathrm{Fr}$ & 6.5 & As \\
\hline 221 & SCHULINE & 0 & NA & $\mathrm{C} 1$ & $005-12$ & $\operatorname{mix} 10 y r 5 / 6,5 / 2$ & $\mathrm{Cl}$, till & $2 \mathrm{msbk}$ & Firm & 7 & As \\
\hline
\end{tabular}




\begin{tabular}{|c|c|c|c|c|c|c|c|c|c|c|c|}
\hline Site & Series & Slope & Aspect & Horizon & Depth & Moist Color & Texture & Structure & Consistency & $\mathrm{Ph}$ & Boundary \\
\hline 225 & SCHULINE & 2 & NA & $\mathrm{B} / \mathrm{C} 1$ & $13-30$ & $10 \mathrm{yr} 4 / 4,10 \mathrm{yr} 4 / 6$ & Sicl & $\mathrm{cp}$ & Vfi & 5.5 & Cs \\
\hline 225 & SCHULINE & 2 & NA & $\mathrm{B} / \mathrm{C} 2$ & $30-44$ & $10 \mathrm{yr} 5 / 2,10 \mathrm{yr} 4 / 6,60 \mathrm{yr} 4 / 4$ & Sicl & $\mathrm{cp}$ & Vfi & 5.5 & Cs \\
\hline 225 & SCHULINE & 2 & NA & $\mathrm{B} / \mathrm{C} 3$ & $44-60$ & 10yr5/6, coal mix 2.5yr5/1 & L & $\mathrm{m}$ & Efi & 6 & Cs \\
\hline 226 & LENZBURG & 28 & NA & $A$ & $0-4$ & $10 y r 4 / 3$ & Sil & $1 \mathrm{fgr}$ & $\mathrm{Fr}$ & 7 & As \\
\hline 226 & LENZBURG & 28 & NA & $\mathrm{B} / \mathrm{C} 1$ & $004-19$ & $10 y r 5 / 2 m$ 10yr $4 / 3,10 y r 5 / 6$ & Sicl & $\mathrm{cp}$ & $\mathrm{Fi}$ & $7.8, \mathrm{FC}$ & Cs \\
\hline 226 & LENZBURG & 28 & NA & $\mathrm{B} / \mathrm{C} 2$ & $19-36$ & $10 \mathrm{yr} 5 / 6,10 \mathrm{yr} 4 / 4,2.5 \mathrm{yr} 5 / 2$ & Sicl & $\mathrm{cp}$ & Vfi & $8, \mathrm{FC}$ & Cs \\
\hline 226 & LENZBURG & 28 & NA & $\mathrm{B} / \mathrm{C} 3$ & $36-60$ & $2.5 y r 5 / 1$ mix coal $10 y r$ 5/6 & L & $\mathrm{m}$ & Efi & $8, \mathrm{FC}$ & NA \\
\hline 229 & SCHULINE & 1 & NA & $A$ & $0-4$ & $10 y r 4 / 3$ & Sil & $1 \mathrm{fgr}$ & $\mathrm{Fr}$ & 6 & As \\
\hline 229 & SCHULINE & 1 & NA & $\mathrm{A} / \mathrm{B}$ & $004-8$ & $10 y r 4 / 3,10 y r 4 / 6$ & Sil & $1 \mathrm{fsbk}$ & $\mathrm{Fr}$ & 6.5 & As \\
\hline 229 & SCHULINE & 1 & NA & $\mathrm{B} / \mathrm{C} 1$ & $008-30$ & $10 y r 5 / 3,5 y r 5 / 2,10 y r 4 / 4$ & Sicl & $\mathrm{cp}$ & $\mathrm{Fi}$ & $8, \mathrm{FC}$ & Cs \\
\hline 229 & SCHULINE & 1 & NA & $\mathrm{B} / \mathrm{C} 2$ & $30-46$ & $10 y r 4 / 410 y r 4 / 2$ & Sicl & $\mathrm{cp}$ & Vfi & $8, \mathrm{FC}$ & Cs \\
\hline 229 & SCHULINE & 1 & NA & $\mathrm{B} / \mathrm{C} 3$ & $46-60$ & $\mathrm{~N} \mathrm{2/0} \mathrm{10yr} \mathrm{4/4}$ & $\mathrm{Cl}$ & $\mathrm{m}$ & Efi & $8, \mathrm{FC}$ & NA \\
\hline 230 & SCHULINE & 1 & NA & $A$ & $0-9$ & $10 y r 4 / 3$ & Sil & $1 \mathrm{fgr}$ & $\mathrm{Fr}$ & 6 & As \\
\hline 230 & SCHULINE & 1 & NA & $\mathrm{B} / \mathrm{C} 1$ & $009-36$ & $10 \mathrm{yr} 4 / 3,2.5 \mathrm{yr} 5 / 1,10 \mathrm{yr} 4 / 6$ & Sicl & $\mathrm{cp}$ & Vfi & $8, \mathrm{FC}$ & As \\
\hline 230 & SCHULINE & 1 & NA & $\mathrm{B} / \mathrm{C} 2$ & $36-60$ & $10 y r 5 / 6, N 2 / 0,5 G 5 / 4$ & $\mathrm{cl}$ & $\mathrm{cp}$ & Efi & 5 & NA \\
\hline 231 & SCHULINE & 0 & NA & Ap & $0-4$ & $10 y r 2 / 2,10 y r 4 / 3$ & Sil & $1 \mathrm{fgr}$ & $\mathrm{Fr}$ & 6 & As \\
\hline 231 & SCHULINE & 0 & NA & $\mathrm{B} / \mathrm{C} 1$ & $004-9$ & $7.5 y r$ 5/6, $10 y r$ 5/2, $10 y r 4 / 6$ & Sil & $1 \mathrm{fgr}$ & $\mathrm{Fr}$ & 6 & As \\
\hline 231 & SCHULINE & 0 & NA & $\mathrm{B} / \mathrm{C} 2$ & $009-60$ & $7.5 \mathrm{yr} 5 / 6,10 \mathrm{yr} 4 / 4, \mathrm{~N} 2 / 05 \mathrm{yr} 6 / 1$ & $\mathrm{~L} / \mathrm{CL}$ & $\mathrm{m}$ & Efi & $8, \mathrm{FC}$ & NA \\
\hline
\end{tabular}




\begin{tabular}{|c|c|c|c|c|c|c|c|c|c|c|c|}
\hline Site & Series & Slope & Aspect & Horizon & Depth & Moist Color & Texture & Structure & Consistency & $\mathbf{P h}$ & Boundary \\
\hline 232 & SCHULINE & 0 & NA & $A$ & $0-5$ & $10 y r 4 / 3$ & Sil & $1 \mathrm{fgr}$ & $\mathrm{Fr}$ & 6 & As \\
\hline 232 & SCHULINE & 0 & NA & $\mathrm{C}$ & $005-60$ & $7.5 \mathrm{yr} 5 / 8, \mathrm{~N} 1 / 0,5 \mathrm{yr} 6 / 1$ & $\mathrm{~L} / \mathrm{CL}$ & $\mathrm{m}$ & Efi & 7,FC.5 & NA \\
\hline 233 & LENZBURG & 3 & $\mathrm{~N}$ & $\mathrm{AP}$ & $0-5$ & $10 y r 5 / 2$ & Sil & $1 \mathrm{fgr}$ & $\mathrm{Fr}$ & 6.5 & As \\
\hline 233 & LENZBURG & 3 & $\mathrm{~N}$ & $\mathrm{~B} / \mathrm{C} 1$ & $005-19$ & $10 y r 5 / 4,5 / 1,2.5 y r 6 / 2$ & $\mathrm{Cl}, \mathrm{Sicl}$ & $\mathrm{cp}$ & Firm & 6.5 & As \\
\hline 233 & LENZBURG & 3 & $\mathrm{~N}$ & $\mathrm{~B} / \mathrm{C} 2$ & $19-40$ & $\operatorname{mix} 10 y r 5 / 6,5 / 2,5 / 8$ & $\mathrm{Cl}$, till & $\mathrm{cp}$ mass & Firm & 7.,FC5 & As \\
\hline 235 & SCHULINE,NC & 3 & W & $\mathrm{AP}$ & $0-5$ & $10 \mathrm{yr} 4 / 2 \mathrm{~F} 2 /$ conc & Sil & $1 \mathrm{fgr}$ & $\mathrm{Fr}$ & 6 & As \\
\hline 235 & SCHULINE,NC & 3 & W & $\mathrm{C} 1$ & $005-12$ & $10 y r 5 / 2$, cfd $10 y r 5 / 8,4 / 0$ & Sil & mass $\mathrm{cp}$ & Firm & 5.5 & As \\
\hline 235 & SCHULINE,NC & 3 & W & $\mathrm{C} 2$ & $012-32$ & $10 \mathrm{yr} 5 / 4,4 / 14 \mathrm{cf}$, few $5 / 2$ mott. & Sicl & $\mathrm{cp}, 2 \mathrm{msbk}$ & Firm & 5.5 & As \\
\hline 235 & SCHULINE,NC & 3 & W & $\mathrm{C} 3$ & $32-60$ & $\operatorname{mix} 10 y r 5 / 4$, sil, $10 y r 4 / 4$ sicl & Sicl, Sil & mass $\mathrm{cp}$ & Firm & 5.5 & As \\
\hline 237 & SCHULINE,NC & 0 & NA & $A$ & $0-6$ & $\operatorname{mix} 10 y r 4 / 2,4 / 4$ & Sil & $1 \mathrm{fgr}$ & $\mathrm{Fr}$ & 6.4 & Cs \\
\hline 237 & SCHULINE,NC & 0 & NA & $\mathrm{B} / \mathrm{C} 1$ & $006-14$ & $10 y r 5 / 1,10 y r 6 / 8$ & Sil & $\mathrm{cp}$ & $\mathrm{Fr}$ & 6.8 & Cs \\
\hline 237 & SCHULINE,NC & 0 & NA & $\mathrm{B} / \mathrm{C} 2$ & $14-22$ & $\operatorname{mix} 10 y r 4 / 2,4 / 4,5 / 8$ & $\mathrm{Cl}$ & $\mathrm{cp}$ & Firm & 6.8 & Cs \\
\hline 237 & SCHULINE,NC & 0 & NA & $\mathrm{B} / \mathrm{C} 3$ & $22-38$ & $\operatorname{mix} 10 y r 5 / 1$ cfd $10 y r 5 / 4,5 / 8$ & Sicl & $\mathrm{cp}$ & Firm & 6.8 & Cs \\
\hline 237 & SCHULINE,NC & 0 & NA & $\mathrm{B} / \mathrm{C} 4$ & $38-70$ & $\operatorname{mix} 10 y r 5 / 4,5 / 1,5 / 8$ & $\mathrm{Cl}$ & $\mathrm{cp}$ & Firm & 7 & Cs \\
\hline 238 & SCHULINE & 2 & NA & Ap & $0-7$ & $10 y r 4 / 3$ & Sil & $1 \mathrm{mgr}$ & $\mathrm{Fr}$ & 6.8 & As \\
\hline 238 & SCHULINE & 2 & NA & $\mathrm{B} / \mathrm{C} 1$ & $007-15$ & $10 y r 4 / 3,10 y r 4 / 6$ & Sil & $1 \mathrm{mgr}$ & $\mathrm{Fr}$ & $7.5, \mathrm{FC}$ & As \\
\hline 238 & SCHULINE & 2 & NA & $\mathrm{B} / \mathrm{C} 2$ & $15-44$ & $10 y r 4 / 1,10 y r 5 / 8$ & Sicl & $1 \mathrm{vcp}$ & Vfi & $8, \mathrm{FC}$ & Cs \\
\hline 238 & SCHULINE & 2 & NA & $\mathrm{B} / \mathrm{C} 3$ & $44-60$ & $2.5 y r 5 / 1,10 y r 4 / 4$ & $\mathrm{Cl}$ & $\mathrm{m}$ & Efi & $8, \mathrm{FC}$ & NA \\
\hline
\end{tabular}




\begin{tabular}{|c|c|c|c|c|c|c|c|c|c|c|c|}
\hline Site & Series & Slope & Aspect & Horizon & Depth & Moist Color & Texture & Structure & Consistency & $\mathbf{P h}$ & Boundary \\
\hline 239 & SCHULINE & 3 & NA & $A$ & $0-4$ & $10 y r 5 / 2$ & Sil & $1 \mathrm{fgr}$ & $\mathrm{Fr}$ & $7.5, \mathrm{FC}$ & As \\
\hline 239 & SCHULINE & 3 & NA & $\mathrm{B} / \mathrm{C} 1$ & $004-9$ & $10 y r 5 / 6,10 y r 5 / 1$ & Sil & $1 \mathrm{fgr}$ & $\mathrm{Fr}$ & 7.,FC5 & As \\
\hline 239 & SCHULINE & 3 & NA & $\mathrm{B} / \mathrm{C} 2$ & $009-20$ & $10 y r 5 / 6,10 y r 5 / 1$ & Sicl & $\mathrm{cp}$ & Vfi & $8, \mathrm{FC}$ & Cs \\
\hline 239 & SCHULINE & 3 & NA & $\mathrm{B} / \mathrm{C} 3$ & $20-30$ & $10 y r 5 / 6,2.5 y r 5 / 190 \%$ & Sicl & $\mathrm{cp}$ & Vfi & $8, \mathrm{FC}$ & Cs \\
\hline 239 & SCHULINE & 3 & NA & $\mathrm{B} / \mathrm{C} 4$ & $30-48$ & $2.5 y r 5 / 1,10 y r 4 / 4,10 y r 5 / 6$ & Sic & $\mathrm{cp}$ & Vfi & $8, \mathrm{FC}$ & NA \\
\hline 240 & SCHULINE & 3 & NA & $A$ & $0-3$ & $10 y r 4 / 3$ & Sil & $1 \mathrm{fgr}$ & $\mathrm{Fr}$ & 7 & As \\
\hline 240 & SCHULINE & 3 & NA & $\mathrm{B} / \mathrm{C} 1$ & $003-23$ & $10 y r 4 / 4,10 y r 5 / 8,2.5 y r 5 / 1$ & Sicl & $\mathrm{cp}$ & $\mathrm{Fi}$ & $7.8, \mathrm{FC}$ & Cs \\
\hline 240 & SCHULINE & 3 & NA & $\mathrm{B} / \mathrm{C} 2$ & $23-48$ & $10 y r 5 / 6,2.5 y r 5 / 1,10 y r 4 / 4$ & Sicl & $\mathrm{cp}$ & Vfi & $8, \mathrm{FC}$ & NA \\
\hline 241 & LENZBURG & 0 & NA & $\mathrm{BC}$ & $0-30$ & $2.5 y r 5 / 1,10 y r 4 / 4,10 y r 5 / 660 \%$ & Sicl & $\mathrm{Cp}$ & Vfi & $7.5, \mathrm{FC}$ & As \\
\hline 241 & LENZBURG & 0 & NA & C & $30-60$ & $10 y r 5 / 4$ coal $10 y r 4 / 2$ & $\mathrm{Cl}$ & $\mathrm{m}$ & Efi & $8, \mathrm{FC}$ & NA \\
\hline 242 & SCHULINE & 0 & NA & Ap & $0-4$ & $10 \mathrm{yr} 4 / 3$ & Sil & $1 \mathrm{fgr}$ & $\mathrm{Fr}$ & 6 & As \\
\hline 242 & SCHULINE & 0 & NA & $\mathrm{B} / \mathrm{C} 1$ & 004-18 & $10 y r 4 / 6,10 y r 4 / 4$ & Sicl & $\mathrm{cp}$ & Vfi & $7.5, \mathrm{FC}$ & As \\
\hline 242 & SCHULINE & 0 & NA & $\mathrm{B} / \mathrm{C} 2$ & $18-60$ & coal, $10 y r$ 4/2, 2.5yr4/4 & $\mathrm{Cl}$ & $\mathrm{m}$ & Efi & $8, \mathrm{FC}$ & NA \\
\hline 243 & SCHULINE & 0 & NA & A & $0-4$ & $10 y r 4 / 3$ & Sil & $1 \mathrm{fgr}$ & $\mathrm{Fr}$ & $7.5, \mathrm{FC}$ & As \\
\hline 243 & SCHULINE & 0 & NA & $\mathrm{B} / \mathrm{C} 1$ & 004-12 & $10 y r 4 / 2,10 y r 4 / 6$ & Sicl & $\mathrm{cp}$ & Vfi & $8, \mathrm{FC}$ & As \\
\hline 243 & SCHULINE & 0 & NA & $\mathrm{B} / \mathrm{C} 2$ & $012-60$ & coal, $10 y r$ 4/2, 2.5yr 4/4 & $\mathrm{Cl}$ & $\mathrm{m}$ & Efi & $8, \mathrm{FC}$ & NA \\
\hline 246 & SCHULINE,NC & 3 & $\mathrm{~N}$ & AP & $0-4$ & $10 y r 4 / 2$, cf $10 y r 5 / 6$ & Sil & $1 \mathrm{fgr}$ & $\mathrm{Fr}$ & 6 & As \\
\hline 246 & SCHULINE,NC & 3 & $\mathrm{~N}$ & $\mathrm{~B} / \mathrm{C} 1$ & $004-9$ & $\operatorname{mix} 10 y r 5 / 4,5 / 2,5 / 8$ & Cl till & $\mathrm{cp}$ & Firm & 7 & As \\
\hline
\end{tabular}




\begin{tabular}{|c|c|c|c|c|c|c|c|c|c|c|c|}
\hline Site & Series & Slope & Aspect & Horizon & Depth & Moist Color & Texture & Structure & Consistency & $\mathbf{P h}$ & Boundary \\
\hline 246 & SCHULINE,NC & 3 & $\mathrm{~N}$ & $\mathrm{~B} / \mathrm{C} 2$ & $009-24$ & $\operatorname{mix} 10 y r 5 / 1,5 / 2,4 / 6$ & $\mathrm{Cl}$ till & $\mathrm{cp}$ & Firm & $7.5, \mathrm{FC}$ & As \\
\hline 246 & SCHULINE,NC & 3 & $\mathrm{~N}$ & $\mathrm{~B} / \mathrm{C} 3$ & $24-36$ & $\operatorname{mix} 10 y r 5 / 6,5 / 2,4 / 6$ & $\mathrm{Cl}$ till & $\mathrm{cp}$ & Firm & 5.5 & As \\
\hline 246 & SCHULINE,NC & 3 & $\mathrm{~N}$ & $\mathrm{~B} / \mathrm{C} 4$ & $36-60$ & $\operatorname{mix} 10 y r 5 / 1,5 / 6,5 / 2$ & I\&cl & $\mathrm{cp}$ & Vfirm & 5.5 & NA \\
\hline 247 & SCHULINE & 2 & $\mathrm{~N}$ & AP & $0-6$ & $10 y r 5 / 4$, cfd, $10 y r 5 / 2$ & $\mathrm{Cl}$ till & $\mathrm{cp}$ & Firm & 5.5 & As \\
\hline 247 & SCHULINE & 2 & $\mathrm{~N}$ & $\mathrm{~B} / \mathrm{C} 1$ & $006-10$ & $\operatorname{mix} 5 y r 5 / 1,10 y r 5 / 6$ & $\mathrm{Cl}$ till & $\mathrm{cp}$ & Firm & 6.5 & As \\
\hline 247 & SCHULINE & 2 & $\mathrm{~N}$ & $\mathrm{~B} / \mathrm{C} 2$ & $010-22$ & $\operatorname{mix} 10 y r 5 / 6,5 / 2$ & sicl & $1 \mathrm{msbk}, \mathrm{cp}$ & Firm & $7.5, \mathrm{FC}$ & As \\
\hline 247 & SCHULINE & 2 & $\mathrm{~N}$ & $\mathrm{~B} / \mathrm{C} 3$ & $22-60$ & $\operatorname{mix} 10 y r 5 / 6,5 / 1,5 / 8$ & $\mathrm{Cl}$ till & $\mathrm{cp}$ & Firm & $7.5, \mathrm{FC}$ & NA \\
\hline 248 & SCHULINE,NC & 1 & $\mathrm{E}$ & A & $0-6$ & $10 y r 5 / 4,10 y r 4 / 2$ & Sil & $1 \mathrm{msbk}$ & $\mathrm{Fr}$ & 5.5 & As \\
\hline 248 & SCHULINE,NC & 1 & $E$ & $\mathrm{C} 1$ & $006-19$ & $10 y r 5 / 4$ mif $10 y r 5 / 6,5 / 2$ & Sicl & $2 \mathrm{msbk}, \mathrm{cp}$ & $\mathrm{Fr}$ & 5.5 & As \\
\hline 248 & SCHULINE,NC & 1 & $E$ & $\mathrm{C} 2$ & $19-32$ & $10 y r 5 / 2$ mid $10 y r$ & Sil & $2 \mathrm{msbk}, \mathrm{cp}$ & $\mathrm{Fr}$ & 6 & As \\
\hline 248 & SCHULINE,NC & 1 & $\mathrm{E}$ & $\mathrm{C} 3$ & $32-55$ & $10 y r 5 / 2,10 y r 4 / 6,5 / 8$ & Sil & $1 \mathrm{msbk}, \mathrm{cp}$ & $\mathrm{Fr}$ & 6 & As \\
\hline 248 & SCHULINE,NC & 1 & $\mathrm{E}$ & C4 & $55-62$ & $5 y r 5 / 1$ cfd $10 y r 4 / 6,5 / 8$ & Loam & massive & $\mathrm{Fr}$ & 6.2 & NA \\
\hline 249 & SCHULINE & 6 & $\mathrm{~N}$ & AP & $0-4$ & $10 y r 4 / 2$ & Sil & $1 \mathrm{fgr}$ & $\mathrm{Fr}$ & 6 & As \\
\hline 249 & SCHULINE & 6 & $\mathrm{~N}$ & $\mathrm{C} 1$ & $004-8$ & $10 y r 5 / 4,10 y r 5 / 8,10 y r 4 / 4 \mathrm{cf}$ & $\mathrm{Cl}$ till & mass $\mathrm{cp}$ & Firm & 6.2 & As \\
\hline 249 & SCHULINE & 6 & $\mathrm{~N}$ & $\mathrm{C} 2$ & $008-30$ & $10 y r 5 / 4,2.5 y r 5 / 2$ & $\mathrm{Cl}$ till & mass $\mathrm{cp}$ & Firm & 6.5 & As \\
\hline 249 & SCHULINE & 6 & $\mathrm{~N}$ & C3 & $30-60$ & $\mathrm{~N} 2 / 0$ coal, $10 \mathrm{yr} 5 / 4,5 / 8$ till & cl $15-20 \%$ & mass $\mathrm{cp}$ & Firm & $7.5, \mathrm{FC}$ & NA \\
\hline 250 & NON-CAL SCHULINE & 1 & $E$ & AP & $0-7$ & $10 y r 4 / 2$ & Sil & $2 f s b k$ & $\mathrm{Fr}$ & 6 & AS \\
\hline 250 & NON-CAL SCHULINE & 1 & $\mathrm{E}$ & $\mathrm{C} 1$ & $007-16$ & $10 y r 5 / 4$, cmd $10 y r 5 / 2,5 / 8$ & Sil & $\mathrm{cp}, 2 \mathrm{msbk}$ & $\mathrm{Fr}$ & 6 & AS \\
\hline
\end{tabular}




\begin{tabular}{|c|c|c|c|c|c|c|c|c|c|c|c|}
\hline Site & Series & Slope & Aspect & Horizon & Depth & Moist Color & Texture & Structure & Consistency & $\mathbf{P h}$ & Boundary \\
\hline 250 & NON-CAL SCHULINE & 1 & $E$ & $\mathrm{C} 2$ & $16-30$ & $2.5 \mathrm{yr} 5 / 2,10 \mathrm{yr} 5 / 8,5 / 4$ & Sil & $2 m s b k, c p$ & Firm & 6 & AS \\
\hline 250 & NON-CAL SCHULINE & 1 & $E$ & $\mathrm{C} 3$ & $30-50$ & $2.5 \mathrm{yr} 6 / 2,10 \mathrm{yr} 5 / 8,5 / 4$ & Sil & $1 \mathrm{msbk}, \mathrm{cp}$ & Firm & 6 & AS \\
\hline 250 & NON-CAL SCHULINE & 1 & $E$ & $\mathrm{C} 4$ & $50-70$ & $10 \mathrm{yr} 5 / 4, \mathrm{cmd} 10 \mathrm{yr} 5 / 4,5 / 2$ & $\mathrm{Cl}$ & $2 \mathrm{msbk}, \mathrm{cp}$ & Firm & 6 & As \\
\hline 251 & SCHULINE & 0 & NA & $A$ & $0-8$ & $\operatorname{mix} 10 y r 5 / 2,4 / 2$ & Sil & $1 \mathrm{fgr}$ & $\mathrm{Fr}$ & 6.2 & As \\
\hline 251 & SCHULINE & 0 & NA & $\mathrm{B} / \mathrm{C} 1$ & $008-18$ & $\operatorname{mix} 10 y r 5 / 3$ cf $5 / 2,5 / 6$ & Hvy Sicl & $\mathrm{cp}$ & Firm & $7.5 \mathrm{FC}$ & As \\
\hline 251 & SCHULINE & 0 & NA & $\mathrm{B} / \mathrm{C} 2$ & $18-26$ & $2.5 y r 6 / 2$ cf $10 y r 5 / 4,5 / 8$ & Sic & $\mathrm{cp}$ & Firm & $7.5 \mathrm{FC}$ & As \\
\hline 251 & SCHULINE & 0 & NA & $\mathrm{B} / \mathrm{C} 3$ & $26-53$ & $\operatorname{mix} 2.5 y r 6 / 2,10 y r 5 / 8$ & sil, sicl & $\mathrm{cp}$ & Firm & $7.5 \mathrm{FC}$ & As \\
\hline 251 & SCHULINE & 0 & NA & $\mathrm{B} / \mathrm{C} 4$ & $53-80$ & $\operatorname{mix} 10 y r 5 / 1,5 / 6$ & $\mathrm{Cl}$ & $\mathrm{cp}$ & Firm & $7.5 \mathrm{FC}$ & As \\
\hline 252 & SCHULINE & 0 & NA & AP & $0-8$ & $10 y r 4 / 3$ & Sil & $1 \mathrm{mgr}$ & $\mathrm{Fr}$ & $7.8 \mathrm{FC}$ & As \\
\hline 252 & SCHULINE & 0 & NA & $\mathrm{B} / \mathrm{C} 1$ & $008-19$ & $10 y r 5 / 3,10 y r 4 / 6$ & Sicl & $f v c p$ & $\mathrm{Fi}$ & $8 \mathrm{fc}$ & Cs \\
\hline 252 & SCHULINE & 0 & NA & $\mathrm{B} / \mathrm{C} 2$ & $19-45$ & $2.5 y r 5 / 1,10 y r 5 / 6$ & Sicl & fvcp & Vfi & $8 \mathrm{fc}$ & CS \\
\hline 252 & SCHULINE & 0 & NA & $\mathrm{B} / \mathrm{C} 3$ & $45-60$ & $2.5 y r$ 5/6, $10 y r$ 4/4, 2.5yr5/1 & $\mathrm{Ch} \mathrm{Cl}$ & $\mathrm{m}$ & efi & $8 \mathrm{fc}$ & NA \\
\hline 253 & SCHULINE & 0 & NA & $A$ & $0-6$ & $10 y r 5 / 4,10 y r 5 / 2$ & Sil & $1 \mathrm{fgr}$ & $\mathrm{Fr}$ & 7 & as \\
\hline 253 & SCHULINE & 0 & NA & $\mathrm{B} / \mathrm{C} 1$ & $006-18$ & $10 y r 5 / 1,10 y r 5 / 6$ & Sicl & $\mathrm{cp}$ & $\mathrm{Fi}$ & $8 \mathrm{fc}$ & Cs \\
\hline 253 & SCHULINE & 0 & NA & $\mathrm{B} / \mathrm{C} 2$ & $18-46$ & $7.5 y r 5 / 6,2.5 y r 5 / 1,10 y r 5 / 6$ & Sicl & $\mathrm{cp}$ & Efi & $8 \mathrm{fc}$ & Cs \\
\hline 253 & SCHULINE & 0 & NA & $\mathrm{B} / \mathrm{C} 3$ & $46-60$ & $2.5 y r 5 / 1,10 y r 5 / 6$, mix coal & L & $\mathrm{m}$ & Efi & $8 \mathrm{fc}$ & Cs \\
\hline 254 & LENZBURG & 4 & NA & $\mathrm{B} / \mathrm{C} 1$ & $0-14$ & $10 y r 5 / 1,10 y r 5 / 680 \%$ & Sicl & $\mathrm{cp}$ & Vfi & 7 fc5 & NA \\
\hline 254 & LENZBURG & 4 & NA & $\mathrm{B} / \mathrm{C} 2$ & $14-30$ & $10 y r 4 / 4,10 y r 5 / 6,2.5 y r 5 / 1$ & Sicl & $\mathrm{cp}$ & Vfi & $8 \mathrm{fc}$ & Cs \\
\hline
\end{tabular}




\begin{tabular}{|c|c|c|c|c|c|c|c|c|c|c|c|}
\hline Site & Series & Slope & Aspect & Horizon & Depth & Moist Color & Texture & Structure & Consistency & $\mathbf{P h}$ & Boundary \\
\hline 254 & LENZBURG & 4 & NA & $\mathrm{B} / \mathrm{C} 3$ & $30-50$ & $2.5 y r 5 / 1,10 y r 5 / 6$ & Sicl & $\mathrm{cp}$ & Vfi & $8 \mathrm{fc}$ & Cs \\
\hline 255 & SCHULINE & 0 & NA & $A$ & $0-16$ & $10 y r 5 / 4,10 y r 5 / 2$ & Sil & $1 \mathrm{fgr}$ & $\mathrm{Fr}$ & $7 \mathrm{fc} 5$ & As \\
\hline 255 & SCHULINE & 0 & NA & $\mathrm{B} / \mathrm{C} 1$ & $016-26$ & $10 y r 4 / 3,10 y r 5 / 6$ & Sicl & $\mathrm{cp}$ & $\mathrm{Fi}$ & $8 \mathrm{fc}$ & Cs \\
\hline 255 & SCHULINE & 0 & NA & $\mathrm{B} / \mathrm{C} 2$ & $26-34$ & $10 y r 4 / 3,5 y r 5 / 1,10 y r 5 / 4$ & Sicl & $\mathrm{cp}$ & Vfi & $8 \mathrm{fc}$ & Cs \\
\hline 255 & SCHULINE & 0 & NA & $\mathrm{B} / \mathrm{C} 3$ & $34-60$ & $10 y r 5 / 2,10 y r 5 / 6$ mix coal & L & $\mathrm{m}$ & Efi & $8 \mathrm{fc}$ & NA \\
\hline 256 & SCHULINE & 1 & NA & $A$ & $0-9$ & $10 y r 5 / 2,10 y r 4 / 3$ & Sil & $1 \mathrm{fgr}$ & $\mathrm{Fr}$ & $8 \mathrm{fc}$ & as \\
\hline 256 & SCHULINE & 1 & NA & $\mathrm{A} / \mathrm{C}$ & $009-16$ & $10 y r 5 / 6,10 y r 4 / 3$ & Sil & $1 \mathrm{fgr}$ & $\mathrm{Fr}$ & $8 \mathrm{fc}$ & cs \\
\hline 256 & SCHULINE & 1 & NA & $\mathrm{B} / \mathrm{C} 1$ & $16-45$ & $2.5 y r 5 / 1,10 y r 5 / 3,10 y r 5 / 6$ & Sicl & $\mathrm{cp}$ & Vfi & $8 \mathrm{fc}$ & cs \\
\hline 256 & SCHULINE & 1 & NA & $\mathrm{B} / \mathrm{C} 2$ & $45-60$ & $10 y r 4 / 4,10 y r 4 / 2$ & $\mathrm{Cl}$ & $\mathrm{m}$ & Efi & $8 \mathrm{fc}$ & NA \\
\hline 257 & SCHULINE & 0 & NA & $A$ & $0-11$ & $10 y r 4 / 3,10 y r 5 / 2$ & Sil & $1 \mathrm{fgr}$ & $\mathrm{Fr}$ & 6.5 & as \\
\hline 257 & SCHULINE & 0 & NA & $\mathrm{B} / \mathrm{C} 1$ & $011-26$ & $10 y r 5 / 2,10 y r 4 / 4,10 y r 5 / 6$ & Sicl & $\mathrm{cp}$ & $\mathrm{Fi}$ & 6 & cs \\
\hline 257 & SCHULINE & 0 & NA & $\mathrm{B} / \mathrm{C} 2$ & $26-42$ & $10 y r 4 / 4,7.5 y r$ 5/6, 2.5yr 5/1 & Sicl & $\mathrm{cp}$ & Vfi & 6 & cs \\
\hline 257 & SCHULINE & 0 & NA & $\mathrm{B} / \mathrm{C} 3$ & $42-60$ & coal 10yr 4/6, 10yr 5/1 & $\mathrm{Cl}$ & & Efi & $7.5 \mathrm{fc}$ & NA \\
\hline 258 & SCHULINE & 0 & NA & $A$ & $0-4$ & $10 y r 4 / 4$ & Sil & $1 \mathrm{fgr}$ & $\mathrm{Fr}$ & 6 & as \\
\hline 258 & SCHULINE & 0 & NA & $\mathrm{B} / \mathrm{C} 1$ & 004-24 & $10 y r 4 / 4,10 y r 4 / 6$ & Sicl & $\mathrm{cp}$ & $\mathrm{Fi}$ & 5.5 & as \\
\hline 258 & SCHULINE & 0 & NA & $\mathrm{B} / \mathrm{C} 2$ & $24-40$ & $2.5 \mathrm{yr}, 10 \mathrm{yr} 4 / 6,10 \mathrm{yr} 4 / 4$ & Sicl & $\mathrm{cp}$ & Vfi & 5.5 & cs \\
\hline 258 & SCHULINE & 0 & NA & $\mathrm{B} / \mathrm{C} 3$ & $40-60$ & $10 y r 4 / 4$ coal, $10 y r 4 / 2$ & $\mathrm{Cl}$ & $\mathrm{m}$ & Efi & $8 \mathrm{fc}$ & NA \\
\hline 259 & SCHULINE & 3 & W & AP & $0-9$ & $\operatorname{mix} 10 y r 4 / 270 \%, 5 / 225 \%, 5 / 6$ & Sil & $1 \mathrm{fsbk}$ & $\mathrm{Fr}$ & 6.5 & as \\
\hline
\end{tabular}




\begin{tabular}{|c|c|c|c|c|c|c|c|c|c|c|c|}
\hline Site & Series & Slope & Aspect & Horizon & Depth & Moist Color & Texture & Structure & Consistency & $\mathbf{P h}$ & Boundary \\
\hline 259 & SCHULINE & 3 & W & $\mathrm{B} / \mathrm{C} 1$ & 009-18 & $\operatorname{mix} 10 y r 5 / 2,5 / 6,6 / 4$ & Sicl & $\mathrm{cp}, \mathrm{sbk}$ & Firm & 5.5 & as \\
\hline 259 & SCHULINE & 3 & W & $\mathrm{B} / \mathrm{C} 2$ & $018-23$ & $\operatorname{mix} 5 y r 5 / 1,2.5 y r 6 / 2,10 y r 5 / 6$ & $\mathrm{Cl}$ & $\mathrm{cp}, \mathrm{sbk}$ & Vfirm & $7.5 \mathrm{fc}$ & as \\
\hline 259 & SCHULINE & 3 & W & $\mathrm{B} / \mathrm{C} 3$ & $23-60$ & $\operatorname{mix} 2.5 y r 6 / 2,10 y r 5 / 6,5 / 4$ & $\mathrm{Cl} \& \mathrm{~L}$ & $\mathrm{cp}, \mathrm{sbk}$ & Vfirm & $7 \mathrm{fc} 5$ & NA \\
\hline 260 & SCHULINE & 0 & NA & AP & $0-7$ & $10 y r 4 / 2,10 y r 5 / 8$ & Sil & $1 \mathrm{mgr}$ & $\mathrm{Fr}$ & 7.5 & as \\
\hline 260 & SCHULINE & 0 & NA & C1 & $007-18$ & $10 y r 5 / 3,10 y r 5 / 3,5 / 810 y r 4 / 2$ mix & Sil, Cl & $\mathrm{cp}$ & $\mathrm{Fr}$ & 7 & as \\
\hline 260 & SCHULINE & 0 & NA & $\mathrm{C} 2$ & $18-32$ & $10 y r 5 / 2$, cf $2.5 y r 6 / 2,10 y r 5 / 8$ & Sicl & $\mathrm{cp}$ & Firm & 5.5 & As \\
\hline 260 & SCHULINE & 0 & NA & C3 & $32-41$ & 10yr6/2, mmd 10yr5/8, 5/2 & Sicl & $\mathrm{cp}$ & Firm & 5.5 & as \\
\hline 260 & SCHULINE & 0 & NA & $\mathrm{C} 4$ & $41-58$ & $10 y r 5 / 4, m m d$ 10yr5/1,5/8 & $\mathrm{Cl}$ & $\mathrm{cp}$ & Firm & 5.5 & as \\
\hline 260 & SCHULINE & 0 & NA & C5 & $58-75$ & $10 y r 4 / 3$, cf10yr5/6, 5/2 & $\mathrm{Cl}$ & $\mathrm{cp}$ & Firm & 7 & NA \\
\hline 261 & HICKORY & 0 & NA & AP & $0-6$ & 10yr4/2, 10yr3/2, 10yr5/4, subsoil & Sil & $2 \mathrm{fgr}$ & $\mathrm{Fr}$ & 5.5 & As \\
\hline 261 & HICKORY & 0 & NA & Bt1 & 006-12 & $10 \mathrm{yr} 5 / 4,4 / 4 \mathrm{cf}$ cont. & Sicl & $2 m s b k$ & $\mathrm{Fr}$ & 6 & Cs \\
\hline 261 & HICKORY & 0 & NA & Bt2 & $012-18$ & $7.5 y r 5 / 4,10 y r 4 / 4 \mathrm{cf}$ & Sicl & $2 m s b k$ & $\mathrm{Fr}$ & 6 & cs \\
\hline 261 & HICKORY & 0 & NA & Bt3 & $18-30$ & $10 y r 5 / 4, w 10 y r 5 / 4$ cf & $\mathrm{Cl}$ & $2 m s b k$ & Firm & 6.2 & cs \\
\hline 261 & HICKORY & 0 & NA & Bt4 & $30-50$ & $10 y r 4 / 4$ cf $10 y r 5 / 1$ & $\mathrm{Cl}$ & $2 m s b k$ & Firm & 6.5 & cs \\
\hline 261 & HICKORY & 0 & NA & C & $50-70$ & 10yr4/2, wmm 5yr 5/1, 10yr5/8 & $\mathrm{Cl}$ & massive & Firm & 7.5 & NA \\
\hline 262 & SCHULINE & 3 & $\mathrm{NE}$ & AP & $0-8$ & $10 y r 5 / 3$, cfd10yr 5/6, mix w/10yr & Sil & $1 \mathrm{fsbk}$ & $\mathrm{Fr}$ & 7 & as \\
\hline 262 & SCHULINE & 3 & NE & $\mathrm{C} 1$ & $008-17$ & $10 y r 5 / 4$, cmd $10 y r 5 / 1,10 y r 5 / 8$ & $\mathrm{Cl}$ & $\mathrm{cp}$ & Firm & 7 & as \\
\hline 262 & SCHULINE & 3 & $\mathrm{NE}$ & $\mathrm{C} 2$ & $17-32$ & $10 y r 5 / 1$ cmd 10yr 5/4 & $\mathrm{Cl}$ & $\mathrm{cp}$ & Firm & $7.5 \mathrm{fc}$ & as \\
\hline
\end{tabular}




\begin{tabular}{|c|c|c|c|c|c|c|c|c|c|c|c|}
\hline Site & Series & Slope & Aspect & Horizon & Depth & Moist Color & Texture & Structure & Consistency & $\mathbf{P h}$ & Boundary \\
\hline 262 & SCHULINE & 3 & NE & $\mathrm{C} 3$ & $32-52$ & $10 y r 5 / 4,5 / 1$ & $\mathrm{Cl}$ & $\mathrm{cp}$ & Firm & 7.5 f.c & as \\
\hline 262 & SCHULINE & 3 & NE & $\mathrm{C} 4$ & $52-80$ & $\operatorname{mix} 10 y r 5 / 4,5 / 210 y r 5 / 8$ & $\mathrm{Cl}$ & $\mathrm{cp}$ & Firm & $7.5 \mathrm{fc}$ & NA \\
\hline 263 & SWANWICK & 1 & $\mathrm{~N}$ & AP & $0-9$ & $10 y r 4 / 2,5 / 6$ & Sil & $2 \mathrm{fgr}$ & $\mathrm{Fr}$ & 6 & cs \\
\hline 263 & SWANWICK & 1 & $\mathrm{~N}$ & $\mathrm{~B} / \mathrm{C} 1$ & $007-15$ & $10 y r 5 / 2,6 / 2,5 / 8$ & Sil & $1 \mathrm{fsbk}$ & $\mathrm{Fr}$ & 6 & cs \\
\hline 263 & SWANWICK & 1 & $\mathrm{~N}$ & $\mathrm{~B} / \mathrm{C} 2$ & $015-30$ & $10 y r 5 / 1,6 / 8,4 / 6$ & Sil & $1 \mathrm{msbk}$ & $\mathrm{Fr}$ & 6 & cs \\
\hline 263 & SWANWICK & 1 & $\mathrm{~N}$ & $\mathrm{~B} / \mathrm{C} 3$ & $30-60$ & $10 y r 6 / 1,6 / 8,4 / 6$ & Sil & $1 \mathrm{msbk}$ & $\mathrm{Fr}$ & 6 & cs \\
\hline 264 & SCHULINE & 1 & $\mathrm{~N}$ & AP & $0-8$ & $10 y r 4 / 2,10 y r 5 / 4$ subsoil & Sil & $1 \mathrm{mgr}$ & $\mathrm{Fr}$ & 7 & as \\
\hline 264 & SCHULINE & 1 & $\mathrm{~N}$ & $\mathrm{~B} / \mathrm{C} 1$ & $008-14$ & $10 y r 5 / 1,10 y r 5 / 8,5 / 3$ & Sil & $\mathrm{cp}$ & $\mathrm{Fr}$ & 7 & as \\
\hline 264 & SCHULINE & 1 & $\mathrm{~N}$ & $\mathrm{~B} / \mathrm{C} 2$ & $14-29$ & $10 y r 5 / 4$, cf $10 y r 5 / 8,5 / 1$ & $\mathrm{Cl}$ & $\mathrm{cp}$ & Firm & 7 & as \\
\hline 264 & SCHULINE & 1 & $\mathrm{~N}$ & $\mathrm{~B} / \mathrm{C} 3$ & $29-43$ & $10 y r 6 / 3,10 y r 5 / 1,10 y r 5 / 8$ & $\mathrm{Cl}$ & $\mathrm{cp}$ & Firm & 7 & as \\
\hline 264 & SCHULINE & 1 & $\mathrm{~N}$ & $\mathrm{~B} / \mathrm{C} 4$ & $43-70$ & $10 y r 5 / 4$, cp 5/8, 5/1 & $\mathrm{Cl}$ & $\mathrm{cp}$ & Firm & 7 & NA \\
\hline 265 & na & 1 & NA & AP & $0-4$ & $10 y r 4 / 3$ & Sil & $1 \mathrm{fgr}$ & $\mathrm{Fr}$ & 6 & as \\
\hline 265 & na & 1 & NA & $\mathrm{B} / \mathrm{C} 1$ & $004-9$ & $10 y r 4 / 6,10 y r 4 / 3$ & Sil & $1 \mathrm{fgr}$ & $\mathrm{Fr}$ & 6.5 & as \\
\hline 265 & na & 1 & NA & $\mathrm{B} / \mathrm{C} 2$ & $009-44$ & $2.5 y r 5 / 1,10 y r \quad 4 / 3,10 y r 4 / 6$ & Sicl & ep & Vfi & $8 \mathrm{fc}$ & cs \\
\hline 265 & na & 1 & NA & $\mathrm{B} / \mathrm{C} 3$ & NA & $10 y r 4 / 2, N 2 / 0,10 y r 4 / 6$ & $\mathrm{Cl}$ & $\mathrm{m}$ & Efi & $8 \mathrm{fc}$ & NA \\
\hline 266 & SCHULINE & 0 & NA & $A$ & $0-5$ & $10 y r 4 / 4,10 y r 3 / 2$ & Sil & $1 \mathrm{fgr}$ & $\mathrm{Fr}$ & $7.5 \mathrm{fc}$ & as \\
\hline 266 & SCHULINE & 0 & NA & $\mathrm{A} / \mathrm{C}$ & $005-14$ & $10 y r 4 / 4,10 y r 3 / 2,10 y r 5 / 6$ & Sil & $1 \mathrm{msbk}$ & $\mathrm{Fr}$ & $8 \mathrm{fc}$ & as \\
\hline 266 & SCHULINE & 0 & NA & $\mathrm{B} / \mathrm{C} 1$ & $14-40$ & $10 y r 3 / 2,2.5 y r 5 / 1,10 y r 4 / 6$ & Sicl & $\mathrm{cp}$ & $\mathrm{Fi}$ & $8 \mathrm{fc}$ & cs \\
\hline
\end{tabular}




\begin{tabular}{|c|c|c|c|c|c|c|c|c|c|c|c|}
\hline Site & Series & Slope & Aspect & Horizon & Depth & Moist Color & Texture & Structure & Consistency & $\mathrm{Ph}$ & Boundary \\
\hline 266 & SCHULINE & 0 & NA & $\mathrm{B} / \mathrm{C} 2$ & $40-60$ & coal 2.5yr 5/1 & $\mathrm{L}$ & $\mathrm{m}$ & Efi & $8 \mathrm{fc}$ & NA \\
\hline 267 & HICKORY & 4 & W & AP & $0-3$ & $10 y r 5 / 3$ & $\mathrm{Cl}$ & $1 \mathrm{fsbk}$ & Firm & 7 & as \\
\hline 267 & HICKORY & 4 & W & $\mathrm{Cg} 1$ & 003-9 & $2.5 \mathrm{yr} 5 / 2$, ffd $10 \mathrm{yr} 5 / 8$ & $\mathrm{Cl}$ & $1 \mathrm{msbk}$ & Firm & 7 & cs \\
\hline 267 & HICKORY & 4 & W & $\mathrm{Cg} 2$ & $009-14$ & $2.5 \mathrm{yr} 5 / 2, \mathrm{mmd} 10 \mathrm{yr} 5 / 8$ & $\mathrm{Cl}$ & $1 \mathrm{msbk}$ & Firm & $7.5 \mathrm{fc}$ & cs \\
\hline 267 & HICKORY & 4 & W & $\mathrm{Cg} 2$ & $14-50$ & $2.5 y r 5 / 2 \mathrm{ffd} 10 \mathrm{yr} 5 / 8$ & $\mathrm{~L}$ & massive & Firm & $8 \mathrm{fc}$ & cs \\
\hline 267 & HICKORY & 4 & W & $\mathrm{Cg} 3$ & $50-60$ & $2.5 \mathrm{yr} 6 / 2, \mathrm{mmd} 10 \mathrm{yr} 5 / 8$ & $\mathrm{Cl}$ & massive & Firm & $8 \mathrm{fc}$ & NA \\
\hline 268 & HICKORY & 1 & NA & AP & $0-4$ & $10 y r 4 / 2$ few $10 y r 5 / 8$ & Sil & $1 \mathrm{fgr}$ & $\mathrm{Fr}$ & $7.5 \mathrm{fc}$ & as \\
\hline 268 & HICKORY & 1 & NA & $\mathrm{C} 1$ & $004-14$ & $\operatorname{mix} 10 y r 4 / 2,5 / 2,5 / 6$ & Sil, Sicl & $\mathrm{cp}$ & $\mathrm{Fr}$ & $7.5 \mathrm{fc}$ & as \\
\hline 268 & HICKORY & 1 & NA & $\mathrm{C} 2$ & $14-25$ & $10 y r 4 / 1$ cf $10 y r$ 5/6, 6/1 & $\mathrm{Cl}$ & $\mathrm{cp}$ & Firm & $7.5 \mathrm{fc}$ & as \\
\hline 268 & HICKORY & 1 & NA & C3 & $25-48$ & $10 y r 5 / 4$, few $6 / 1 \mathrm{~cm} 5 / 8$ & $\mathrm{Cl}$ & $\mathrm{cp}$ & Firm & $7.5 \mathrm{fc}$ & as \\
\hline 268 & HICKORY & 1 & NA & $\mathrm{C} 4$ & $48-75$ & $10 y r$ 6/4, cm 10yr 5/8, 5/2 & $\mathrm{Cl}$ & $\mathrm{cp}$ & Firm & $7.5 \mathrm{fc}$ & NA \\
\hline 269 & NON-CAL SCHULINE & 0 & NA & AP & $0-4$ & $10 y r 4 / 2$ & Sil & $1 \mathrm{mgr}$ & $\mathrm{Fr}$ & 6 & as \\
\hline 269 & NON-CAL SCHULINE & 0 & NA & A1 & $004-12$ & $\operatorname{mix} 10 y r 4 / 2,5 / 4$ & Sil & $1 \mathrm{mgr}$ & $\mathrm{Fr}$ & 6 & as \\
\hline 269 & NON-CAL SCHULINE & 0 & NA & $\mathrm{B} / \mathrm{C} 1$ & $012-20$ & $10 y r 5 / 2 \operatorname{mix} 4 / 6,4 / 2$ & Sil & $\mathrm{cp}$ & $\mathrm{Fr}$ & 6 & as \\
\hline 269 & NON-CAL SCHULINE & 0 & NA & $\mathrm{B} / \mathrm{C} 2$ & $20-30$ & $10 y r 4 / 4 \mathrm{~cm} 10 y r 4 / 3$ & $\mathrm{Cl}$ & $\mathrm{cp}$ & $\mathrm{Fr}$ & 6.5 & as \\
\hline 269 & NON-CAL SCHULINE & 0 & NA & $\mathrm{B} / \mathrm{C} 3$ & $30-70$ & $10 y r 5 / 4,10 y r 5 / 8,10 y r 5 / 2$ & $\mathrm{Cl}$ & $\mathrm{cp}$ & Firm & 6.5 & as \\
\hline 270 & NON-CAL LENZBURG & 40 & $\mathrm{E}$ & $\mathrm{AO}$ & $0-9$ & 10yr 4/2 (90\%) 5/6 (10\%) mix & Sil & $1 \mathrm{fgr}$ & $\mathrm{Fr}$ & 6 & as \\
\hline 270 & NON-CAL LENZBURG & 40 & $\mathrm{E}$ & $\mathrm{B} / \mathrm{C} 1$ & $009-20$ & $10 y r 5 / 1,4 / 2,5 / 6$ & Sicl, Sil & $\mathrm{cp}$ & Firm & 6.2 & as \\
\hline
\end{tabular}




\begin{tabular}{|c|c|c|c|c|c|c|c|c|c|c|c|}
\hline Site & Series & Slope & Aspect & Horizon & Depth & Moist Color & Texture & Structure & Consistency & $\mathbf{P h}$ & Boundary \\
\hline 270 & NON-CAL LENZBURG & 40 & E & $\mathrm{B} / \mathrm{C} 2$ & $20-32$ & $2.5 \mathrm{yr} 6 / 2,5 / 6$ & Hvy Cly & $\mathrm{cp}$ & Firm & 6.7 & as \\
\hline 270 & NON-CAL LENZBURG & 40 & $\mathrm{E}$ & $\mathrm{B} / \mathrm{C} 3$ & $32-50$ & 5 yr $5 / 1$, cfd $10 y r 5 / 8,2.5 y r 6 / 2$ & $\mathrm{Cl}$ & $\mathrm{cp}$ & Firm & 6.5 & as \\
\hline 270 & NON-CAL LENZBURG & 40 & $\mathrm{E}$ & $\mathrm{B} / \mathrm{C} 4$ & $50-66$ & $10 y r 5 / 8,2.5 y r 6 / 1$ & $\mathrm{Cl}$ & $\mathrm{cp}$ & Firm & 6.5 & as \\
\hline 271 & NON-CAL SCHULINE & 5 & NA & AP & $0-9$ & 10yr 4/2 fld 10yr 4/6 cmf 10yr 5/2 & Sil & $2 \mathrm{mgr}$ & $\mathrm{Fr}$ & 6 & as \\
\hline 271 & NON-CAL SCHULINE & 5 & NA & $\mathrm{B} / \mathrm{C} 1$ & $009-14$ & 10yr 4/2, 10yr 4/6, 5/2 10yr5/4 & Sil & $2 m g r$ & $\mathrm{Fr}$ & 6 & as \\
\hline 271 & NON-CAL SCHULINE & 5 & NA & $\mathrm{B} / \mathrm{C} 2$ & $14-28$ & 10yr $5 / 4$ cf $10 y r 5 / 2,5 / 8$ 10yr4/2 & Sicl, Sil & $\mathrm{cp}, \mathrm{sbk}$ & $\mathrm{Fr}$ & 6 & as \\
\hline 271 & NON-CAL SCHULINE & 5 & NA & $\mathrm{B} / \mathrm{C} 3$ & $28-38$ & $10 y r 5 / 6,2.5 y r 6 / 2$, cf10yr5/2,5/4 & Sicl & $\mathrm{cp}, \mathrm{sbk}$ & Firm & 6.2 & as \\
\hline 271 & NON-CAL SCHULINE & 5 & NA & $\mathrm{B} / \mathrm{C} 4$ & $38-70$ & $10 y r 5 / 4,5 / 8,5 / 2$ & Sicl & $\mathrm{cp}, \mathrm{sbk}$ & Firm & 6 & as \\
\hline
\end{tabular}




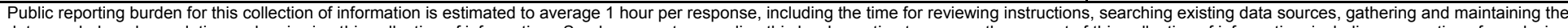

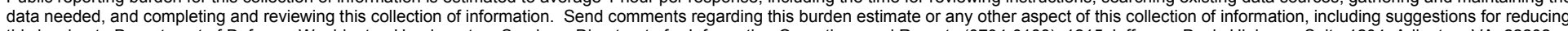

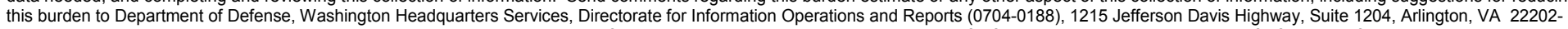

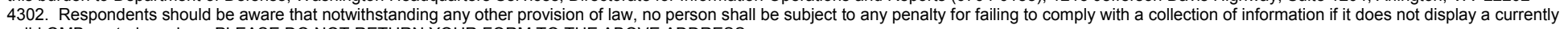
valid OMB control number. PLEASE DO NOT RETURN YOUR FORM TO THE ABOVE ADDRESS.
1. REPORT DATE (DD-MM-YYYY)
2. REPORT TYPE
06-2003
Final
3. DATES COVERED (From - To)

4. TITLE AND SUBTITLE

Initial Assessment of the Soil and Vegetation of the Illinois National Guard Sparta Training Area

5a. CONTRACT NUMBER

5b. GRANT NUMBER

5c. PROGRAM ELEMENT NUMBER

6. AUTHOR(S)

Jeffrey S. Fehmi, Robert L. McLeese, and Jonathan L. Casebeer

5d. PROJECT NUMBER

622720A896

5e. TASK NUMBER

CNN-T081

5f. WORK UNIT NUMBER

\section{PERFORMING ORGANIZATION NAME(S) AND ADDRESS(ES)}

U.S. Army Engineer Research and Development Center (ERDC)

Construction Engineering Research Laboratory (CERL)

PO Box 9005

Champaign, IL 61826-9005

\section{SPONSORING / MONITORING AGENCY NAME(S) AND ADDRESS(ES)}

10. SPONSOR/MONITOR'S ACRONYM(S)

U.S. Army Environmental Center

5179 Hoadley Road

Aberdeen Proving Ground, MD 21010-5401

11. SPONSOR/MONITOR'S REPORT NUMBER(S)

\section{DISTRIBUTION / AVAILABILITY STATEMENT}

Approved for public release; distribution is unlimited.

\section{SUPPLEMENTARY NOTES}

Copies are available from the National Technical Information Service, 5285 Port Royal Road, Springfield, VA 22161.

\section{ABSTRACT}

The Illinois National Guard (ILNG) is acquiring a new 2800-acre training area near Sparta, Illinois. This acquisition is important in that it allows the National Guard units in southern Illinois a readily available place to train, which will increase training effectiveness and save time and money through decreased travel costs associated with using the existing training area in the northern part of the state.

The recent acquisition of the Sparta training area represents a unique opportunity to gather baseline data before any training takes place. This data will be valuable in that it gives the Army the unique opportunity to learn about the conditions before and after training as well as strengthening any future empirically collected research data. This represents a fundamental knowledge gap in much of the current research on Army lands and represents a high priority, high payoff area of research.

The initial plant and soil data were collected using a grid-based sampling protocol to allow uniform and unbiased cover. The specific sampling protocols for each type of data follows in the vegetation and soils sections and the data are included in the appendices.

\section{SUBJECT TERMS}

environmental management; land management; Illinois National Guard; soils; vegetation; Sparta, IL

\begin{tabular}{|c|c|c|}
\hline \multicolumn{3}{|c|}{ 16. SECURITY CLASSIFICATION OF: } \\
\hline $\begin{array}{l}\text { a. REPORT } \\
\text { Unclassified }\end{array}$ & $\begin{array}{l}\text { b. ABSTRACT } \\
\text { Unclassified }\end{array}$ & $\begin{array}{l}\text { c. THIS PAGE } \\
\text { Unclassified }\end{array}$ \\
\hline
\end{tabular}
17. LIMITATION
OF ABSTRACT

SAR
18. NUMBER OF PAGES

114 19a. NAME OF RESPONSIBLE PERSON Jeffrey S. Fehmi

19b. TELEPHONE NUMBER (include area code)

(217) 352-6511, ext 6366 